\title{
Comparing Baseband and Intermediate Frequency FMCW Radar Receivers
}

\author{
by
}

\author{
Bernard Meyer
}

Thesis presented in partial fullfilment of the requirements for the degree of Master of Science in Engineering at the

Faculty of Electrical and Electronic Engineering at Stellenbosch University

Supervisors: Prof. J. B. de Swardt

Dr. P. van der Merwe

December 2015 


\section{Declaration}

By submitting this thesis electronically, I declare that the entirety of the work contained therein is my own, original work, that I am the sole author thereof (save to the extent explicitly otherwise stated), that reproduction and publication thereof by Stellenbosch University will not infringe any third party rights and that I have not previously in its entirety or in part submitted it for obtaining any qualification.

Signature:

Date:

B Meyer

Copyright (c) 2015 Stellenbosch University

All rights reserved 


\section{Abstract}

Frequency modulated continuous wave (FMCW) radars have a large range of applications in the civilian and military sectors, including aircraft navigation (radio altimeters), subsurface examination, weather monitoring, and many more remote sensing applications. In general receivers have limited dynamic range. Therefore, FMCW radar receivers can saturate due to large reflections from close range targets or poor isolation between the radar system's transmit and receive paths.

It is hypothesised that certain radar receiver topologies are more suited to coping with saturation. Therefore, the intermediate frequency (IF) and the commonly-used baseband (or zero-IF) FMCW radar receiver topologies are compared in terms of component nonlinearity. Receiver saturation generates unwanted signals that can result in the detection of false targets. The study proposes that an IF receiver, under specific operating conditions, can suppress unwanted harmonics and intermodulation products (IMPs) much more effectively than an equivalent baseband receiver.

A detailed IF receiver design is presented that includes a discussion on image rejection, signal quantization, anti-aliasing, and sensitivity frequency control.

It is found that the IF receiver is immune to the detection of false targets caused by poor isolation between the demodulator's local oscillator and radio frequency ports.

A noise analysis shows that the IF receiver's noise performance is potentially greater than that of an equivalent baseband receiver due to pink noise that has a $1 / f$ power spectral density, which decreases with an increase in operating frequency.

The non-linear behaviour of the IF receiver is investigated by analysing the circuit components that have the highest risk of saturation in the receiver chain. The magnitude of harmonics and IMPs are determined using an algebraic approximation of a non-linear model presented in the study. The model is used to predict under which conditions false targets will be detected.

It is found that the zero-IF receiver is susceptible to distortions that are suppressible by an IF receiver. Though the application has limitations with regards to the received signal characteristics, the IF receiver has better or equivalent performance when compared to a baseband receiver. 


\section{Uittreksel}

Frekwensie gemoduleerde kontinue golf (FMCW) radars het verskye toepassings in die privaat en militêre sektore, insluitend vliegtuig navigasie stelsels (radio altimeters), grond penetrasie radars, weer monitors, en nog vele afstandswaarnemingstoepassings. In die algemeen het ontvangers 'n beperkte dinamiese bereik. Dus kan FMCW radar ontvangers versadig as gevolg van groot weerkaatsings vanaf naby geleë teikens of swak isolasie tussen die radarstelsel se transmissie en ontvang paaie.

Dit word gepostuleer dat sekere radar ontvanger topologieë meer geskik is vir die hantering van ontvanger versadiging. Daarom word die intermediêre frekwensie (IF) en die meer algemene basisband FMCW radar ontvanger topologieë vergelyk in terme van komponent nie-lineariteit. Ontvanger versadiging genereer ongewenste seine wat as vals teikens gesien kan word. Die studie wys dat 'n IF ontvanger ongewenste harmonieke en intermodulasie produkte (IMPs) kan onderdruk wat 'n soortgelyke basisband ontvanger nie kan nie.

'n Gedetailleerde IF ontvanger ontwerp word voorgelê met 'n bespreking oor beeld verwerping, sein kwantisering, anti-aliasering, en frekwensie sensitiwiteit beheer.

Met die gebruik van 'n IF ontvanger is dit bevind dat vals teikens weens swak isolasie tussen die demodulator se plaaslike ossillator en radio frekwensie poorte nie veroorsaak kan word nie.

Die ontvanger ruis analiese toon dat die IF ontvanger beter ruis gedrag het as 'n ekwivalente basisband ontvanger, omdat pienk ruis met 'n $1 / f$ drywingsdigtheidspektrum verminder met 'n toename in frekwensie.

Die nie-lineêre gedrag van die IF ontvanger word ondersoek deur die komponente met die hoogste risiko van versadiging in die ontvanger-ketting te ontleed. Die grootte van harmonieke en IMPs word bepaal met behulp van 'n algebraïese uitbreiding wat 'n nielineêre model beskryf. Hierdie model is toegepas om te voorspel onder watter voorwaardes vals teikens bespeur sal word.

Dit is bevind dat die basisband ontvanger vatbaar is vir distorsies wat die IF ontvanger kan onderdruk. Alhoewel die toepassing beperkings het met betrekking tot die ontvangssein se eienskappe, is die IF ontvanger beter of gelykstaande aan 'n soortgelyke basisband ontvanger. 


\section{Acknowledgements}

I would like to thank the following people for their contribution to this project:

My supervisor, Prof. J. B. de Swardt, for your insights and guidance, Paul van der Merwe for providing expert advice, Elmine Botes for your help with revision and support throughout the project, my family for their encouragement, and Reutech Radar Systems for funding the project. 


\section{Contents}

Declaration $\quad$ i

Abstract

Uittreksel iii

Acknowledgements $\quad$ iv

List of Figures $\quad$ viii

List of Tables $\quad$ xiv

Nomenclature $\quad$ xvi

1 Introduction 1

1.1 Problem statement . . . . . . . . . . . . . . . . . . . . . 1

1.2 Objective of this study . . . . . . . . . . . . . . . . . . . . . . . . . . . . . . . . .

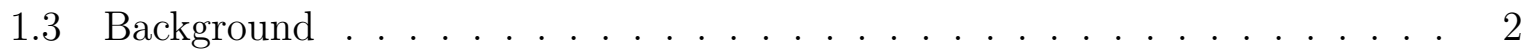

1.4 Project overview . . . . . . . . . . . . . . . . 7

2 System design overview $\quad 8$

2.1 System block diagram . . . . . . . . . . . . . . . . . . . . 9

2.2 Sawtooth frequency modulation . . . . . . . . . . . . . . . . . 19

2.3 Conclusion . . . . . . . . . . . . . . . . . . . . 20

3 Receiver design $\quad 21$

3.1 Intermediate frequency receiver . . . . . . . . . . . . . . . . 21

3.2 Image signals and image rejection . . . . . . . . . . . . . . . . . 23

3.3 Selecting an analog-to-digital converter . . . . . . . . . . . . . . 30

3.4 Anti-aliasing filters in undersampling applications . . . . . . . . . . . 35

3.5 Intermediate frequency filter . . . . . . . . . . . . . . . 36

3.6 Anti-aliasing filter . . . . . . . . . . . . . . . . . . . . . . 57

3.7 Conclusion . . . . . . . . . . . . . . . . . . . . . . . . 59

4 Receiver noise $\quad 60$

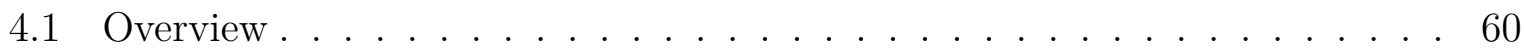

4.2 Thermal noise . . . . . . . . . . . . . . . . . . 63

4.3 Noise figure . . . . . . . . . . . . . . . . . . . . . . 66

4.4 Adding noise sources . . . . . . . . . . . . . . . . . . . . 69

4.5 Equivalent noise bandwidth . . . . . . . . . . . . . . . 70 
4.6 Amplifier noise . . . . . . . . . . . . . . . . . . . . . . . . . 71

4.7 Noise analysis . . . . . . . . . . . . . . . . . . . . . . . . . . . 81

4.8 Conclusion . . . . . . . . . . . . . . . . . . . . . . . . . . . . . . . . . . 91

5 Receiver non-linearity $\quad 93$

5.1 Linearity figure of merit . . . . . . . . . . . . . . . . . . . . 94

5.2 Receiver dynamic range . . . . . . . . . . . . . . . . . . . . . . 95

5.3 Representing non-linear responses . . . . . . . . . . . . . . . . . . . . . . . 99

5.4 Low noise amplifier non-linearity . . . . . . . . . . . . . . . . . . 102

5.5 Quadrature demodulator non-linearity . . . . . . . . . . . . . . . 104

5.6 Approximate device non-linearity . . . . . . . . . . . . . . . 107

5.7 Conclusion . . . . . . . . . . . . . . . . . . . . 121

6 Receiver characteristics and dynamic range 123

6.1 Windowing of discrete signals . . . . . . . . . . . . . . . . . . . . . . 124

6.2 Receiver characterisation . . . . . . . . . . . . . . . . . 125

6.3 Demodulator LO to RF leakage . . . . . . . . . . . . . . . . . . . 131

6.4 Conclusion . . . . . . . . . . . . . . . . . . . . . . 132

7 Measurements and results 133

7.1 Receiver noise figure measurement . . . . . . . . . . . . . . . . . . . 133

7.2 Time domain interferometry measurements . . . . . . . . . . . . . . 136

7.3 Conclusion . . . . . . . . . . . . . . . . . . . . . . . . . . . . 143

8 Conclusions and recommendations $\quad 144$

8.1 Research . . . . . . . . . . . . . . . . . . . . . . . . . . 144

8.2 Findings and recommendations . . . . . . . . . . . . . . . . . . . . . . . . . . . . . . . . . . . . . .

8.3 Conclusion . . . . . . . . . . . . . . . . . . . . . 145

A Receiver theory 146

A.1 Down conversion . . . . . . . . . . . . . . . . . 146

A.2 Algebraic formula to determine the amplitudes of intermodulation products and harmonics caused by an arbitrary number of carriers . . . . . . . . . 146

B Matlab code $\quad 151$

B.1 Synthesis of a general Chebyshev filter function . . . . . . . . . . . . 151

B.2 Synthesis of the fully-canonical coupling matrix . . . . . . . . . . . . 154

B.3 Similarity transformation and matrix reconfiguration . . . . . . . . . . 156

B.4 Coupling matrix to lumped elements . . . . . . . . . . . . . . . 159

B.5 Noise figure and Dynamic range calculation code . . . . . . . . . . . . 161

B.6 LT6200 non-linear simulation code . . . . . . . . . . . . . . . . . . . . . . . . . . . . . . . . . . . .

B.7 Receiver calibration code . . . . . . . . . . . . . . . . . . 173

$\begin{array}{lr}\text { C Notation } & 174\end{array}$

$\begin{array}{ll}\text { D Miscellaneous measurements } & 176\end{array}$

$\begin{array}{lr}\text { E Datasheet information } & 177\end{array}$

$\begin{array}{lr}\text { F Schematics and PCB layouts } & 179\end{array}$ 
F.1 Demodulator baseband circuit . . . . . . . . . . . . . . . . . 179

F.2 Intermediate frequency offset local oscillator circuit . . . . . . . . . 179

G Photos 


\section{List of Figures}

1.1 A single large reflection causes the radar receiver to saturate, generating undesired harmonics: (a) unwanted distortions fall outside the IF receiver's passband, (b) the same reflection received by a zero-IF receiver results in the detection of false targets. . . . . . . . . . . . . . . . . . 2

1.2 Radar classification according to the type of transmitted signal. . . . . . . . . 3

1.3 Diagram of a FMCW radar using a single antenna configuration with a circulator. 4

1.4 Frequency versus time plot for the sawtooth FM scheme, where the transmitted and received signals are shown. Two reflections are depicted, with their beat frequencies $f_{b 1}$ and $f_{b 2}$ respectively, where the red line represents the target nearest to the radar. . . . . . . . . . . . . . . . . 5

1.5 Triangular frequency modulation of a transmitted carrier signal and its received echo with the down converted beat frequencies. . . . . . . . . . . 5

1.6 Frequency versus time plot for the IF sawtooth FM scheme, where the IF offset LO, transmitted and received signals are shown. Two reflections are depicted, with their beat frequencies $f_{b 1}$ and $f_{b 2}$ respectively, where the red line represents the target nearest to the radar. . . . . . . . . . . . 6

2.1 Block diagram of the IF FMCW radar. . . . . . . . . . . . . . . . . . 8

2.2 Transmitter circuit block diagram. . . . . . . . . . . . . . . . . . . . . . . . . . . . . . . . . . . . .

2.3 DDS amplitude response. . . . . . . . . . . . . . . . . . 10

2.4 Intermediate frequency offset local oscillator circuit block diagram. . . . . . . 11

2.5 Second order equal-ripple Chebyshev bandpass filter circuit. . . . . . . . . . . 11

2.6 Second order equal-ripple Chebyshev bandpass filter amplitude response. . . . 12

2.7 Multi-stage passive polyphase filter topology. . . . . . . . . . . . . . . . 13

2.8 (a) First order passive polyphase filter. (b) Simplified first order passive polyphase filter. . . . . . . . . . . . . . . . . 13

2.9 A first order passive polyphase filter's magnitude and phase response. . . . . . 14

2.10 (a) IQ modulator functional block diagram. (b) Simplified IQ modulator block diagram. . . . . . . . . . . . . . . . . . . 15

2.11 (a) Passive polyphase filter and quadrature modulator configuration. (b) Simplified mathematical representation of the modulation scheme with input IF and Tx signals resulting in an IF offset RF output signal. . . . . . . . . . . . . 15

2.12 Receiver circuit block diagram. . . . . . . . . . . . . . . . . 17

2.13 Third-order intercept point for a cascaded system: (a) two component cascaded network, (b) equivalent network. . . . . . . . . . . . . 18

2.14 Tx (black line): sawtooth down chirp, generated by the DDS for transmission. LO (green line): transmission signal with a negative IF offset. Rx (red line): received signal. 
3.1 Block diagram of a zero-IF receiver topology. . . . . . . . . . . . . . . . . . . 22

3.2 Schematic representation of the IF receiver topology. . . . . . . . . . . . . . 23

3.3 Down conversion without image-rejection filter. . . . . . . . . . . . . . . . 24

3.4 Down conversion with image-rejection filter. . . . . . . . . . . . . . . . 24

3.5 (a) Half complex and (b) full complex receiver architectures using polyphase filters. . . . . . . . . . . . . . . . . . 25

3.6 (a) Hartley (Half complex) and (b) Weaver (full complex) image rejection

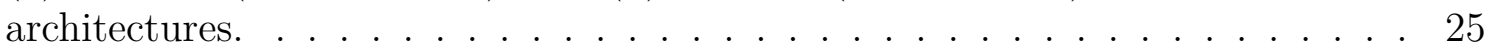

3.7 IRR versus phase error at different gain mismatch values. . . . . . . . . . . . 26

3.8 (a) First order passive polyphase filter excited differentially at both inputs.

(b) Simplified first order passive polyphase filter fed at both inputs. . . . . . . 27

3.9 Normalised image rejection of the upper and lower sidebands. . . . . . . . . . 30

3.10 The Hartley architecture's measured image rejection ratio. . . . . . . . . . . . . 30

3.11 Discrete sampling of an continuous analog signal. . . . . . . . . . . . . . . 31

3.12 Spectral representation of a quantized continuous baseband signal. . . . . . . . 32

3.13 Time domain representation of sampling and aliasing. . . . . . . . . . . . . . 33

3.14 Signal aliased from a higher Nyquist zone to baseband. . . . . . . . . . . . . . 34

3.15 Minimum required sample frequency versus the desired signal's maximum frequency scaled to the signal bandwidth. . . . . . . . . . . . . . . . 34

3.16 Anti-aliasing filter for undersampling. . . . . . . . . . . . . . . 35

3.17 (a) Inductor model, (b) Capacitor model. . . . . . . . . . . . . . . . . . . . . 37

3.18 Representation of the desired intermediate frequency filter characteristics. . . . 38

3.19 The roots of the $E(s), P(s)$ and $F(s)$ polynomials in the complex S-plane. . . 43

3.20 Magnitude of $S_{11}$ and $S_{21}$ of the synthesised Chebyshev filter versus frequency. 44

3.21 General two-port lossless network described by Y-parameters. . . . . . . . . . 44

3.22 Canonical transversal array. (a) $N$-resonator transversal array including direct source-load coupling $M_{S L}$. (b) Equivalent circuit of the $k$-th resonator with coupling in the transversal array. . . . . . . . . . . . . . . . . .

$3.23 N+2$ canonical coupling matrix $\mathbf{M}$ for the transversal array. The matrix is symmetric about the principal diagonal, i.e., $M_{i j}=M_{j i}$. . . . . . . . . . . . . 48

3.24 Example of a sixth degree rotational matrix $\mathbf{R}_{r}$ with pivot point $[2,5]$. The angle $\theta_{r}$ is setup to annihilate entry $M_{S 5} \ldots \ldots \ldots \ldots$

3.25 (a) Idealised filter elements illustrating the loaded Q source impedance $\left(R_{Q S}\right)$, LC-resonator, and the admittance inverter between resonator 1 and 2 . (b) Practical filter elements, matching network between the loaded $\mathrm{Q}$ and desired input impedance $\left(R_{S}\right)$. A capacitive $\Pi$-inverter network replaces the ideal $J$-inverter.

3.26 (a) Operation of the admittance inverter. (b) Implementation of the admittance inverter using a capacitor network.

3.27 (a) IF filter lumped element circuit with ideal admittance inverters, (b) equivalent lumped element model and П-inverters, (c) practical IF filter implementation.

3.28 The IF filter's amplitude response. Shown are the measured results, ideal lumped element simulation, and ESR simulation.

3.29 The IF filter's phase response. Shown are the measured results, the ideal lumped element simulation, and the ESR simulation.

3.30 The IF filter response is transposed to baseband, where zero beat frequency is located at zero frequency. . . . . . . . . . . . . . . . . . . 
3.31 The anti-aliasing filter's amplitude response: measured results, ideal lumped element simulation, and ESR simulation. . . . . . . . . . . . . . .

3.32 The anti-aliasing filter's phase response: measured results, ideal lumped element simulation, and ESR simulation.

4.1 Nyquist's conductor setup for thermal noise description.

4.2 (a) Noisy resistor, (b) Thévenin equivalent circuits, (c) Nortan equivalent circuit. 65

4.3 (a) An arbitrary white noise source generating a noise power of $N_{0}$. (b) An equivalent noise temperature $\left(T_{e}\right)$ and matched impedance $(R)$ to characterise the arbitrary white noise source. . . . . . . . . . . . . . . 66

4.4 Noisy two-port network. . . . . . . . . . . . . . . . . . . . . . . 67

4.5 General $n$ stage cascaded network, where each stage is represented by its gain, noise figure, and equivalent noise temperature. . . . . . . . . . . . . . . . . . 68

4.6 Series noise sources, $\overline{e_{1}^{2}}$, and $\overline{e_{2}^{2}}$, generated by resistors, $R_{1}$, and $R_{2}$. . . . . 70

4.7 Equivalent noise bandwidth (ENB) "brickwall" representation of a system's

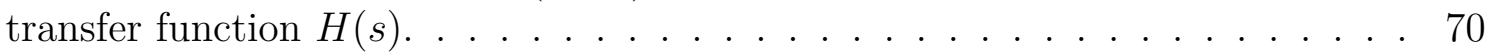

$4.8 e_{n}-i_{n}$ amplifier noise model represented by instantaneous noise sources, a Thévenin equivalent input source is applied to the network. . . . . . . . . . . 72

4.9 Pink and white noise intersect point at $f_{n c}$ on a log-log scale. . . . . . . . . 73

4.10 Linear Technology's LT6200 op-amp's input noise versus frequency plots. . . . 74

4.11 Non-inverting operational amplifier noise model. . . . . . . . . . . . . . . . . 75

4.12 The root-mean-square input referred noise to the non-inverting operational amplifier. . . . . . . . . . . . . . . . 76

4.13 Fully differential operational amplifier circuit noise model. . . . . . . . . . . . 79

4.14 Fully differential operational amplifier circuit noise model with Thévenin equivalent input source. . . . . . . . . . . . . . . . . . . . 79

4.15 System available gain. . . . . . . . . . . . . . . . . . . . . 81

4.16 Receiver chain block diagram. . . . . . . . . . . . . . . . . . . . 82

4.17 RF circuit up to the differential amplifiers at the demodulator output channels I and Q. . . . . . . . . . . . . . . . . . 83

4.18 Fully differential operational amplifier input and output noise sources. . . . . . 85

4.19 Four-port noise representation using Z-parameters. . . . . . . . . . . . . . . 86

4.20 Passive polyphase filter four-port network. . . . . . . . . . . . . . 86

4.21 Received signal and receiver noise at the output of the buffer op-amp C. . . . 88

4.22 IF-filter noise contribution. . . . . . . . . . . . . . . . . . . . . . . . . . 89

4.23 The high voltage gain amplifier's noise sources, labelled D in Fig. 4.16. . . . . 90

4.24 Calculated cascaded noise figure versus frequency. . . . . . . . . . . . . . 91

5.1 FMCW radar IF receiver block diagram. . . . . . . . . . . . . . . . . . 93

5.2 Graphical representation of dynamic range definitions. . . . . . . . . . . . . . 94

5.3 Effective dynamic range. . . . . . . . . . . . . . . . . . . . . . . . . . . . . . 95

5.4 Full scale RF input power and minimum detectable RF signal level versus frequency. The cascaded RF $1 \mathrm{~dB}$ compression point (IP1dB cas $)$ is the upper limit to the cascaded RF component's spurious free dynamic range. The effective dynamic range is the green area between the $F S_{R X}, \mathrm{RF} I \mathrm{IP} 1 \mathrm{~dB}_{\text {cas }}$ and $M D S_{R X}$ lines. . . . . . . . . . . . . . . . . . . . . . 98

5.5 Voltage transfer curve with idealized straight line approximation. . . . . . . . 99

5.6 Spectrum of third order products from two equal-power inputs. . . . . . . . . 101 
5.7 Spectrum of third order products from two unequal-power inputs, where $\delta$ represents the difference in amplitude of the fundamental components. . . . . 101

5.8 LNA measurement set-up. . . . . . . . . . . . . . . . . . . . 103

5.9 The Mini-Circuits ZX60-P105LN+ LNA's output harmonics versus input power.103

5.10 Mini-Circuits ZX60-P105LN+ LNA s-parameters. . . . . . . . . . . . . . . . 104

5.11 The set-up used to measure the Analog Devices ADL5380 quadrature demodulator's non-linear behaviour.

5.12 The ADL5380 quadrature demodulator's measured I-channel output harmonics versus input power.

5.13 The ADL5380 IQ-demodulator measurement set-up circuit model representation used to determine the devices open circuit voltage gain $G_{v \text {,demod }}$. . . . 106

5.14 Receiver chain flow diagram. . . . . . . . . . . . . . . . . . . 108

5.15 Simplified Linear Technology LT6200 schematic. . . . . . . . . . . . . . . . . . 108

5.16 Ideal diode representation. . . . . . . . . . . . . . . . . . . . . . . . . . . . . . . . . . . . . . . . . . . . . . . . . . . . .

5.17 Diode voltage limiter circuit. . . . . . . . . . . . . . . . . . . . . 109

5.18 Linear Technologies LT6200 op-amp harmonic and intermodulation measurement setup. . . . . . . . . . . . . . . . . . . . . . . . . 112

5.19 Fifth-order low-pass filter ladder network. . . . . . . . . . . . . . . . 112

5.20 Fifth-order $3 \mathrm{~dB}$ equal-ripple low-pass filter amplitude response. . . . . . . . . 112

5.21 Measured and simulated 3rd order harmonic distortion $\left(f_{H 3}=3 f_{1}\right)$ versus the input amplitude ratio. . . . . . . . . . . . . . . . . . . . 113

5.22 Measured and simulated spectrum when $P_{1}=P_{2}=3.7 \mathrm{dBm} . \ldots \ldots$. . . . 114

5.23 Measured and simulated IMP3,A $\left(f_{I M P 3, A}=2 f_{1}-f_{2}\right)$ versus the input amplitude ratio. . . . . . . . . . . . . . . . . . 115

5.24 Measured and simulated IMP3,B $\left(f_{I M P 3, B}=2 f_{2}-f_{1}\right)$ versus the input amplitude ratio. . . . . . . . . . . . . . . . . . 115

5.25 The non-linear model's input-to-output voltage curve. . . . . . . . . . . . 116

5.26 Simulated diode voltage limiter circuit. . . . . . . . . . . . . . . 117

5.27 Third-order intermodulation product's voltage level at the ADC's input $\left(f_{I M P 3, B}=\right.$ $\left.2 f_{2}-f_{1}\right)$ versus the input amplitude ratio of the fundamental components. . . 117

5.28 Third-order intermodulation product's voltage level at the ADC's input $\left(f_{I M P 3, A}=\right.$ $\left.2 f_{1}-f_{2}\right)$ versus the input amplitude ratio of the fundamental components. . . 118

5.29 Third-order harmonic's voltage level at the ADC's input $\left(f_{H 3}=3 f_{1}\right)$ versus the input amplitude ratio of the fundamental components. . . . . . . . . . . 119

5.30 Amplitude ratio between the third order harmonic $\left(f_{H 3}=3 f_{1}\right)$ and third order $\operatorname{IMP}\left(f_{I M P 3, A}=2 f_{1}-f_{2}\right)$ versus the input amplitude ratio of the fundamental components. . . . . . . . . . . . . . . . .

5.31 IF receiver spectrum, where the harmonics and IMPs fall outside the band of interest.

IF receiver spectrum, where the fundamental components are detected and the

IMP is below the MDS level. . . . . . . . . . . . . . . . . . . 121

5.33 Baseband receiver spectrum, where the third order harmonic (H3) is detected as a false target. . . . . . . . . . . . . . . . . . 12

6.1 Receiver measurement set-up. . . . . . . . . . . . . . . . . . . . . 123

6.2 Three common windows to reduce spectral leakage. . . . . . . . . . . . . . . 124

6.3 Signal amplitude in ADC quantisation levels versus RF input power in dBm. . 125

6.4 Change in the ADC's quantisation level on a logarithmic scale versus RF input power in $\mathrm{dBm}$. 
6.5 The receiver's normalised amplitude response. . . . . . . . . . . . . . . . 127

6.6 The measured voltage and power gain. . . . . . . . . . . . . . . . . 128

6.7 Time domain measurement of the LT6200 op-amp in saturation. . . . . . . . . 128

6.8 Frequency domain measurement that shows non-linearity at the LT6200 opamp's output. . . . . . . . . . . . . . . . . . . . . . . . . . . . 129

6.9 The undesired harmonics are physically removed by the anti-aliasing filter and a spurious free spectrum is digitised by the ADC . . . . . . . . . . . . . . . . 129

6.10 Measured noise floor. . . . . . . . . . . . . . . . . . . . 130

6.11 False targets caused by LO to RF leakage. . . . . . . . . . . . . . . 132

7.1 Output noise power versus noise source temperature. . . . . . . . . . . . . 133

7.2 Test set-up for the Y factor noise figure measurement method. . . . . . . . . . 134

7.3 Test set-up for the Y factor noise figure measurement using an oscilloscope. . . 135

7.4 Calculated and measured cascaded noise figure versus frequency. . . . . . . . . 136

7.5 Single coaxial transmission line connected between the transmitter and receiver.137

7.6 (a) Measured and simulated spectrum due to a single tone excitation $\left(P_{R x}=\right.$ $-23 \mathrm{~dB}$ ). (b) Close-up spectral view of the measurements, simulation and IF filter response. . . . . . . . . . . . . . . . . . . . . . . . . . 138

7.7 A single tone $\left(P_{R x}=-23 \mathrm{~dB}\right)$ zero-IF receiver simulation is shown along side a measurement made using the IF FMCW radar. The measured spectrum is transposed to baseband by subtracting the IF. . . . . . . . . . . . . . . . . 139

7.8 Parallel transmission lines connected between the transmitter and receiver. . . 140

7.9 (a) The simulated and measured spectrum due to a two tone excitation $\left(P_{R x, 1}=\right.$ $-23 \mathrm{dBm}$ and $P_{R x, 2}=-48 \mathrm{dBm}$ ). (b) Close-up spectral view of the measurements, simulation and IF filter response. . . . . . . . . . . . . . . . . . . 141

7.10 Two tone excitation with: (a) $P_{R x, 1}=-23 \mathrm{dBm}$ and $P_{R x, 2}=-48 \mathrm{dBm}$, (b) $P_{R x, 1}=-23 \mathrm{dBm}$ and $P_{R x, 2}=-58 \mathrm{dBm}$. Comparing the simulated spectrum for a zero-IF receiver to a measurement made using the IF receiver. The measured spectrum is transposed to baseband by subtracting the IF. . . . . . . 142

A.1 The symbolic functional diagram of a signal multiplier (idealized mixer) illustrating frequency down conversion. . . . . . . . . . . . . 146

D.1 One meter coaxial cable insertion loss vs frequency. . . . . . . . . . . 176

F.1 The demodulator's baseband output circuitry with sub-circuit blocks. . . . . . 180

F.2 IF-filter schematic. . . . . . . . . . . . . . . . . . . . . . 181

F.3 Anti-aliasing filter schematic. . . . . . . . . . . . . . . . . . . . . . . . . . . . . . . . . . . . . . . . . .

F.4 Passive polyphase filter schematic. . . . . . . . . . . . . . . . . 182

F.5 Two high voltage gain operational amplifier schematics (a) and (b) are presented, but only (b) is populated on the PCB. . . . . . . . . . . . . . . . 183

F.6 Two regulator options are illustrated on the schematic, but option A is populated on the PCB. . . . . . . . . . . . . . . . . . . . . . . . . . . . . . . . . . . . . . . . . .

F.7 Demodulator baseband circuit PCB top layer. . . . . . . . . . . . . . . . . 185

F.8 Demodulator baseband circuit PCB bottom layer . . . . . . . . . . . . . 185

F.9 IF offset local oscillator circuit schematic. . . . . . . . . . . . . . . . . . 186

F.10 Voltage regulator (a) and 128 prescaler schematic (b) . . . . . . . . . . . . 187

F.11 Prescaler bandpass filter schematic. . . . . . . . . . . . . . . . . 187

F.12 Schematic for the passive polyphase filter with differential inputs and outputs. 187 
F.13 The IF offset local oscillator circuit's PCB layout, where (a) and (b) are the top and bottom views respectively. . . . . . . . . . . . . . . . 188

G.1 Cable measurement set-up. . . . . . . . . . . . . . . . . . . . . . . . . . 189

G.2 Constructed modular IF FMCW radar. . . . . . . . . . . . . . . . . . . 189 


\section{List of Tables}

2.1 Lumped element equations . . . . . . . . . . . . . . . . . . . . . . . . 11

2.2 Second order equal-ripple Chebyshev bandpass filter parameters . . . . . . . . 12

2.3 Low noise amplifier parameters as shown on the manufacturer datasheets, and the SFDR and LDR is calculated for $\Delta f=100 \mathrm{kHz} \ldots \ldots . . . . . . .17$

2.4 IQ demodulator parameters as shown on the manufacturer datasheets, with $f_{L O}=900 \mathrm{MHz}, P_{L O}=0 \mathrm{dBm}$, and the SFDR and LDR is calculated for $\Delta f=100 \mathrm{kHz} \ldots \ldots \ldots \ldots \ldots \ldots \ldots$

2.5 Cascaded network parameters . . . . . . . . . . . . . . . . 19

3.1 Intermediate frequency filter parameters (refer to Fig. 3.18) . . . . . . . . . . 40

3.2 The transversal array's $N+2$ canonical coupling matrix for the IF filter . . . 48

3.3 Similarity transform sequence for the reduction of the transversal matrix . . . 50

3.4 Transformed $N+2$ coupling matrix that has the desired topology and filter characteristics . . . . . . . . . . . . . . . . . 51

3.5 IF filter lumped element values . . . . . . . . . . . . . . . . . 55

4.1 Equivalent noise bandwidth (ENB) coefficients for low-pass filter responses . . 71

4.2 Non-inverting op-amp circuit's feedback network thermal voltage noise . . . . 77

4.3 Output referred op-amp internal noise sources . . . . . . . . . . . . . . . . 77

4.4 Fully differential op-amp circuit's feedback network thermal voltage noise . . . 80

4.5 The fully differential op-amp's internal noise sources referred to the output . . 80

4.6 Measured component specifications . . . . . . . . . . . . . . . 83

4.7 Component specifications from datasheets . . . . . . . . . . . . . . 83

5.1 Calculated receiver dynamic range . . . . . . . . . . . . . . . . . . 97

5.2 Variables used in dynamic range calculation . . . . . . . . . . . . . . . . . . . . . . . . . . . . . . . . . .

5.3 Measured ZX60-P105LN+ LNA parameter . . . . . . . . . . . . . . . . 104

5.4 Measured ADL5380 demodulator parameters with the I and Q output channels differentially loaded by $450 \Omega \ldots$. . . . . . . . . . . . . . 106

5.5 LT6200 op-amp model parameters . . . . . . . . . . . . . . . . . 116

6.1 Characteristics of common non-uniform windows . . . . . . . . . . . . . 124

6.2 Window correction factors . . . . . . . . . . . . . . . . 125

6.3 Receiver characteristics - measured and calculated results . . . . . . . . . . 131

6.4 LO leakage to RF port of the ADL5380 demodulator . . . . . . . . . . . . . . 132

7.1 ENR values for the Agilent Technologies 346B noise source. . . . . . . . . . 135

E.1 TCM1-63AX+, Mini-circuits RF 1:1 Impedance transformer - 10 to $6000 \mathrm{MHz}$, insertion loss data . . . . . . . . . . . . . . . . . . . . 177 
E.2 TC1-1-13M+, Mini-circuits RF 1:1 Impedance transformer - 4.5 to $3000 \mathrm{MHz}$, insertion loss data . . . . . . . . . . . . . . . . . . . 177

E.3 TCM9-1+, Mini-circuits RF 9:1 Impedance transformer - 2 to $280 \mathrm{MHz}$, insertion loss data . . . . . . . . . . . . . . . . . . . . . 178 


\title{
Nomenclature
}

\author{
AC Alternating current \\ AM Amplitude modulation \\ ADC Analog-to-digital converter \\ $\omega$ Angular frequency in $\mathrm{rad} / \mathrm{s}$ \\ BB Baseband \\ BER Bit-error-rate \\ $k$ Boltzmann's constant \\ C Capacitance \\ $f_{0} \quad$ Centre frequency \\ K Coupling coefficient \\ CW Continuous wave \\ $\Omega_{c} \quad$ Cut off frequency \\ dB Decibels \\ DUT Device under test \\ DAC Digital-to-analog converter \\ DC Direct current \\ DDS Direct digital synthesiser
}

EM Electromagnetic

e Electron charge

Eq Equation

ENB Equivalent noise bandwidth

ESR Equivalent series resistance

F Farad

FBW Fractional bandwidth

FM Frequency modulation

FMCW Frequency modulated continuous wave 
FDA Fully differential amplifier

FDOA Fully differential operational amplifier

HD Harmonic distortion

H Henry

IR Image rejection

L Inductance

IC Integrated circuit

IP Intercept point

IF Intermediate frequency

IM Intermodulation

IMD Intermodulation distortion

IMP Intermodulation product

LSB Least significant bit

LO Local oscillator

LSB Lower sideband

LNA Low noise amplifier

MUT Material under test

MDFS Maximum distortion free signal

$\mu m$ Micrometer

mm Millimetre

MDS Minimum detectable signal

MSB Most significant bit

F Noise factor

NF Noise figure

$\Omega \mathrm{Ohm}$

$P_{1 d B}$ One decibel compression point

Op-amp Operational amplifier

OIP3 Output third-order intercept

PPF Passive polyphase filter

PLL Phase lock loop

PSD Power spectral density

PCB Printed circuit board 
PRF Pulse repetition frequency

RCS Radar cross section

RF Radio frequency

Rx Receiver

$S_{11} \quad$ Reflection coefficient

$\omega_{0}$ Resonant frequency

rms Root-mean-square

$f_{s} \quad$ Sample frequency

$t_{s}$ Sample intervals

SNR Signal-to-noise ratio

IIP3 Third input intercept point

$S_{21}$ Transmission coefficient

Tx Transmitter

USB Upper sideband

WSS Wide sense stationary 


\section{Chapter 1}

\section{Introduction}

\subsection{Problem statement}

Frequency modulated continuous wave (FMCW) radars have a large range of applications in the civilian and military sectors, including aircraft navigation (radio altimeters) [1], subsurface examination [2] [3], weather monitoring [1], and many more remote sensing applications. There are however, some limitations to using FMCW radars. One of the most important problems faced by designers is that large reflections desensitise the receiver (this is referred to as the hidden transmitter problem by [4]) and generates spurious signals when the receiver's full scale power level is exceeded. These reflections are commonly caused by large objects with a close proximity to the transmitter, or by insufficient isolation between the transmitting and receiving antennas [5]. In the case of a monostatic radar, the leakage caused by poor isolation resemble close range reflections. These factors have a significant impact on radar performance, and need to be taken into account during the FMCW radar design process.

\subsection{Objective of this study}

The objective of this study is to research and evaluate an FMCW radar receiver with an intermediate frequency (IF) offset. The IF and baseband (or zero-IF) FMCW radar receiver implementations are compared in terms of component non-linearity. The aim is to identify operating conditions that allow receiver components to be used at or above their saturation point without detecting spurious signals. By using an IF receiver under specific operating conditions unwanted harmonics and intermodulation products (IMPs) can be removed. Throughout the study the IF and baseband receiver implementations are compared.

Figure 1.1 (a) illustrates a receiver with an IF that is greater than its baseband bandwidth (BB). It is hypothesised that more of the harmonics and IMPs generated from receiver saturation will fall outside the IF receiver's bandwidth, compared to a zero-IF receiver with an equal baseband bandwidth as in Fig. 1.1 (b). The conditions and limitations to this proposal are subject to receiver parameters, component types, and the characteristics of received signals. 

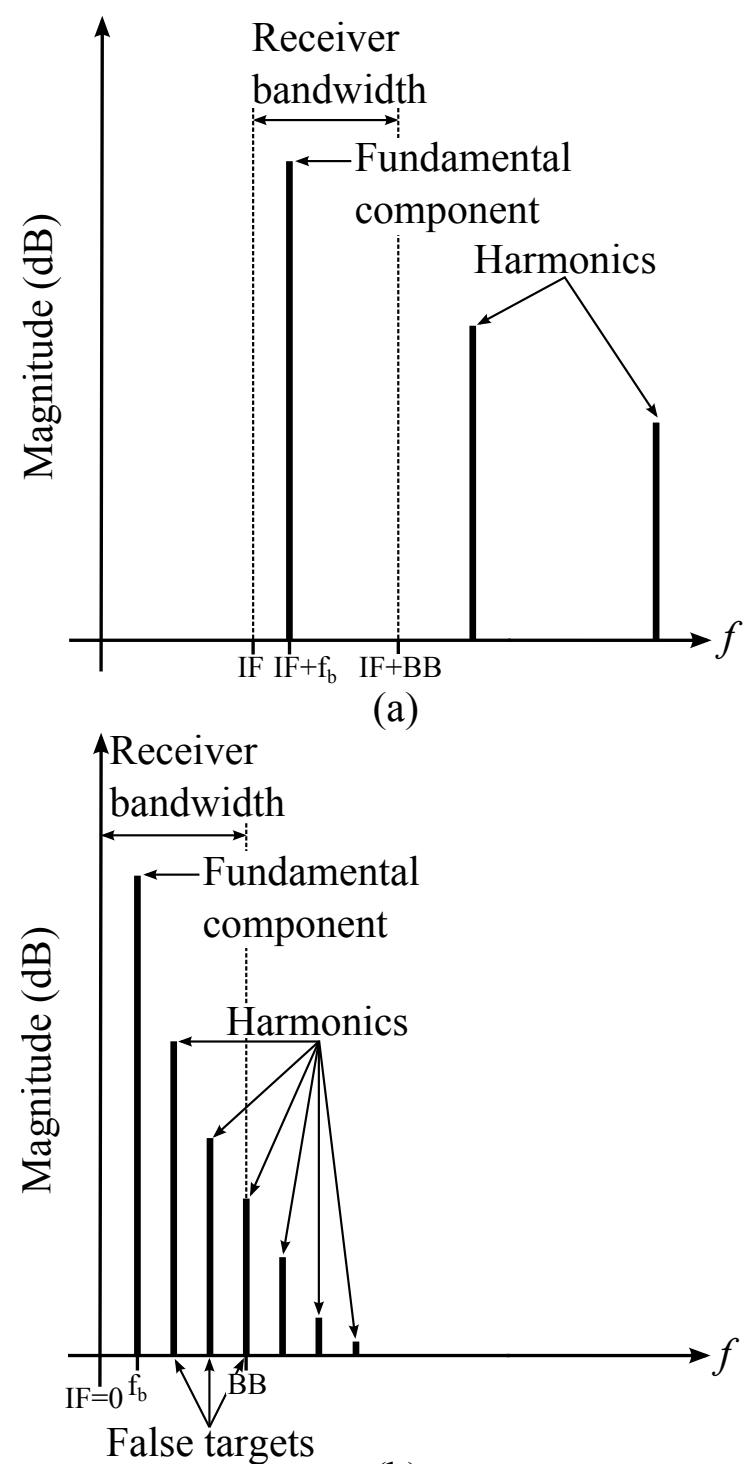

(b)

Figure 1.1: A single large reflection causes the radar receiver to saturate, generating undesired harmonics: (a) unwanted distortions fall outside the IF receiver's passband, (b) the same reflection received by a zero-IF receiver results in the detection of false targets.

\subsection{Background}

In order to fully understand the advantage of using an IF FMCW radar receiver, some background information is required on the common problems faced by an FMCW radar designer. It is also important to understand why an FMCW radar would be chosen over other types of radar systems in specific applications.

\subsubsection{Radar classification}

Radar, as it is known today, was first developed during World War II. The word radar originated in the early 1940's as an abbreviation for radio detection and ranging [1]. As the name suggests, radars are remote sensing devices that acquire information about an object or phenomenon without making physical contact.

Monostatic radar is defined as a radar in which the transmitter and receiver are collo- 
cated, in contrast to multistatic (and bistatic) radar, where the transmitters and receivers are separated. When considering the implementation of a monostatic radar there are different viable options depending on the application. Figure 1.2 provides an overview of the most common kinds of static radars.

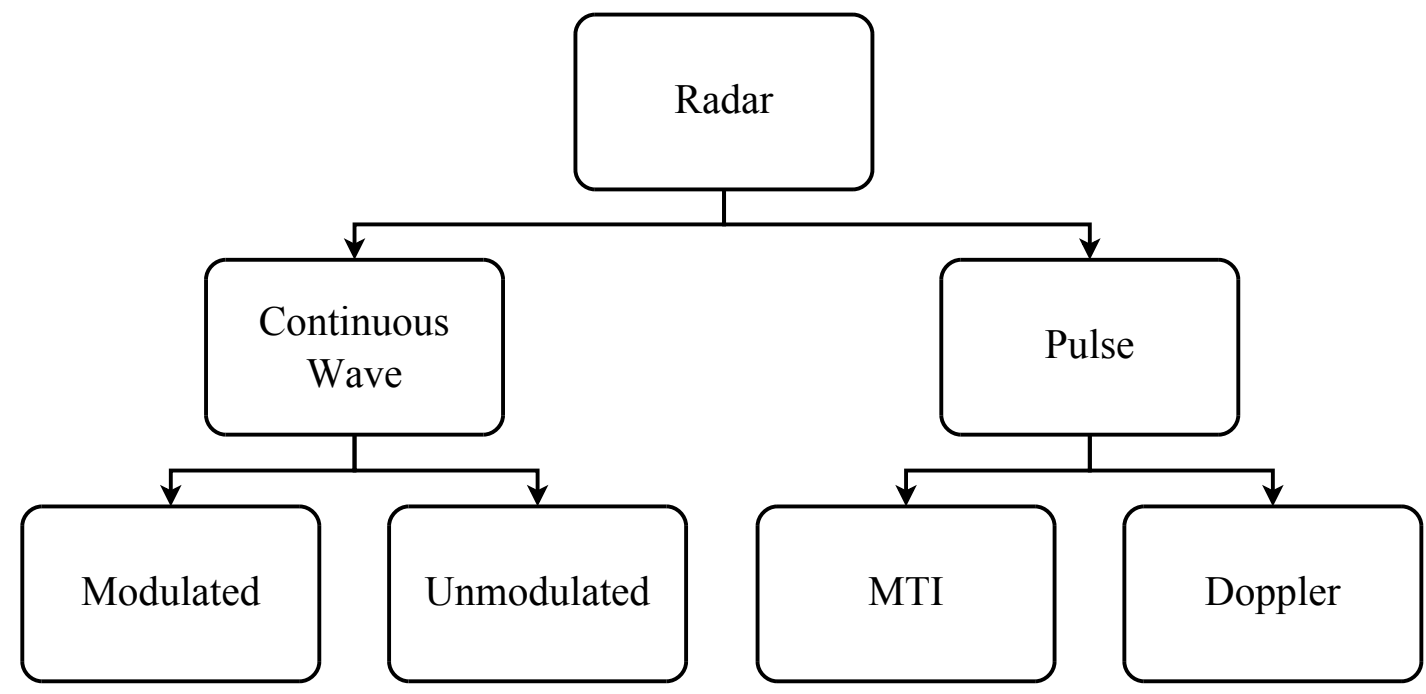

Figure 1.2: Radar classification according to the type of transmitted signal.

A pulse radar transmits a relatively short burst of electromagnetic (EM) energy, and after a fixed delay the receiver is turned on to listen for the echo. The echo not only indicates that a target is present, but provides information about the distance (time delay) and relative velocity (doppler effect) of the target. Moving target indication (MTI) usually refers to a radar in which the pulse repetition frequency (PRF) is chosen low enough to avoid range ambiguities, while pulse doppler radars have high PRF to avoid blind speeds. Range ambiguities occur when a detectable pulse's travel time to an object and back exceeds the time between pulses. Targets with relative velocities that result in doppler frequencies equal to multiples of the PRF produce zero MTI responses, these velocities are called blind speeds [1].

\subsubsection{FMCW radar}

The unmodulated continuous wave $(\mathrm{CW})$ radar is widely used as a method for determining a target's velocity. As the name suggests, a CW radar transmits a sine wave of constant frequency. The $\mathrm{CW}$ radar works by detecting the change in echo-signal frequency caused by the doppler effect when there is relative motion between the radar and its target. However, it is not possible to detect the distance to a target using CW radar, because the time duration between transmission and reception of a signal is indeterminate. The inability of $\mathrm{CW}$ radar to measure distance is due to the narrow bandwidth of the transmitted waveform. The spectrum of a CW transmitter can be broadened by applying the appropriate carrier modulation scheme [1].

Figure 1.3 shows a diagrammatic representation of an FMCW radar with a single antenna that transmits and receives signals. A circulator provides isolation between the transmit $\left(\mathrm{T}_{\mathrm{x}}\right)$ and receive $\left(\mathrm{R}_{\mathrm{x}}\right)$ paths. For the baseband receiver implementation the transmitting signal is used as the local oscillator (LO). The LO is mixed with the received signal resulting in a down converted baseband signal. 


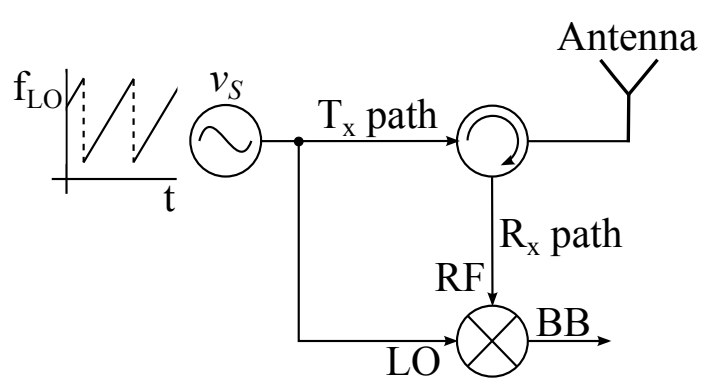

Figure 1.3: Diagram of a FMCW radar using a single antenna configuration with a circulator.

To implement an FMCW radar, the frequency of the transmitted sine wave is modulated by a periodic signal. A change in frequency between the transmitted and received echo signals is observed due to the transmitted signal's frequency modulation (FM) and propagation delay. The most common forms of carrier modulation for FMCW radars are sawtooth, triangular and sinusoidal. Any modulation scheme can be used, but sawtooth FM is chosen in this study. The selection is based on practical considerations discussed in this chapter.

The operation of an FMCW radar is discussed in the context of Fig. 1.3, where the sinusoidal carrier signal generated by the source produces a signal described by the following function

$$
v_{S}(t)=A_{L O} \cos [2 \pi t f(t)],
$$

where the frequency is a periodic sawtooth function given by

$$
\begin{gathered}
f_{\text {saw }}(t)=\frac{\Delta f}{\pi}\left\{\frac{\pi}{2}-\arctan \left[\cot \left(\frac{t \pi}{T_{m}}\right)\right]\right\}, \\
f(t)=f_{\text {saw }}(t)+f_{0} .
\end{gathered}
$$

The frequency bandwidth of the transmitted signal is $\Delta f$, and the modulation period is given by $T_{m}$. Equation (1.3.2) is derived from the Fourier series representation of a periodic sawtooth function.

Figure 1.4 shows the transmitted $\left(\mathrm{T}_{\mathrm{x}}\right)$ and received $\left(\mathrm{R}_{\mathrm{x}}\right)$ signals for two targets detected by a FMCW radar. The round trip delays $\left(\Delta T_{1}\right.$ and $\left.\Delta T_{2}\right)$ for both targets are indicated on the figure. These delays are used to calculate the distance to the targets based on the time difference between transmission and reception of the echo. The round trip time delay is given by Eq. (1.3.4), where $R$ is the distance between the radar and the target, and $v_{p}$ is the propagation velocity of the EM waves,

$$
\Delta T=\frac{2 R}{v_{p}} .
$$

Note that the time delay cannot be determined directly, rather, the difference in frequency is detected and then interpreted to gather range information. Next, the relationship between frequency, time and range is discussed in further detail.

The difference in frequency between the $\mathrm{LO}$ and received signal $\left(R_{x}\right)$ is determined by mixing the LO and radio frequency (RF) signal, resulting in a down converted baseband or IF signal. The process of down conversion is discussed in Appendix A.1.

Both the targets represented by Fig. 1.4 are stationary, because no doppler shift is observed and only a time delay was introduced. Doppler shifts are characterized by 
an increase or decrease in carrier frequency. Since the conclusions drawn for stationary targets also apply to moving targets, the study will for the most part be concerned with static targets.

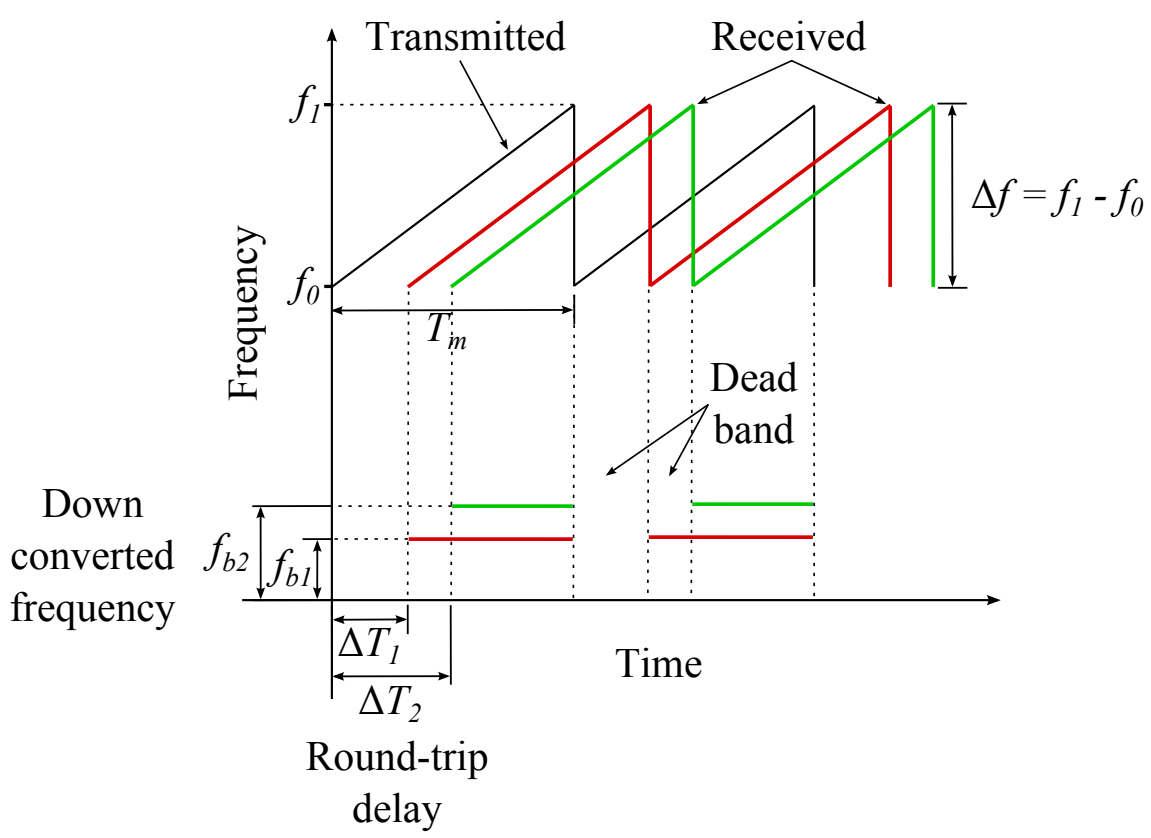

Figure 1.4: Frequency versus time plot for the sawtooth FM scheme, where the transmitted and received signals are shown. Two reflections are depicted, with their beat frequencies $f_{b 1}$ and $f_{b 2}$ respectively, where the red line represents the target nearest to the radar.

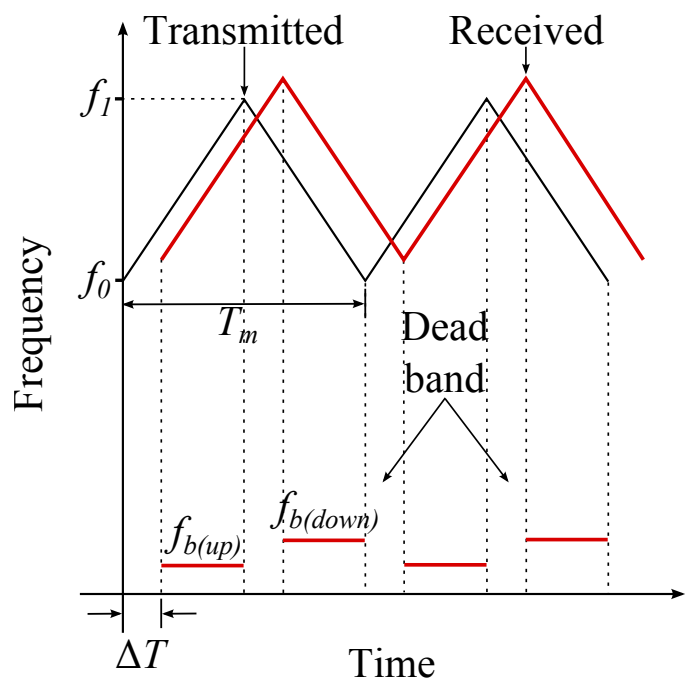

Figure 1.5: Triangular frequency modulation of a transmitted carrier signal and its received echo with the down converted beat frequencies.

Each modulation scheme has its advantages and disadvantages. The chosen sawtooth modulation scheme is ill-suited for the detection of doppler frequencies, but advantageous in receiver sideband suppression. A triangular FM scheme would be more useful in detecting doppler frequencies. As shown by Figure 1.5, when applying triangular FM to a 
carrier, the doppler effect caused by a target in motion produces two distinct beat frequencies, $f_{b(u p)}$, and $f_{b(\text { down })}$, for the same reflection. By processing the difference between these beat frequencies the object's relative velocity can be calculated.

An advantage to using sawtooth FM in the implementation of an IF receiver is that the entire modulation period (excluding the modulation dead-band) is used when one of the sidebands are suppressed. Receiver sideband suppression is a useful tool that increases the receiver sensitivity by rejecting one half of the spectral content within the receiver's band of interest. This topic is further discussed in section 3.2.

If the beat frequency $\left(f_{b}\right)$ caused by a target's echo is known, the range to that target is calculated using Eq. 1.3.5.

$$
R=\frac{f_{b} v_{p} T_{m}}{2 \Delta f}
$$

When deriving Eq. (1.3.5) it is assumed that the modulation dead-band is orders of magnitude shorter than the total modulation period. For most practical systems this assumption is valid and the dead-band is negligible [1]. From Eq. (1.3.5) it is clear that the range to a target is linearly proportional to the beat frequency: low beat frequencies relate to close proximity targets, whereas higher frequencies indicate more distant targets.

\subsubsection{Frequency offset local oscillator}

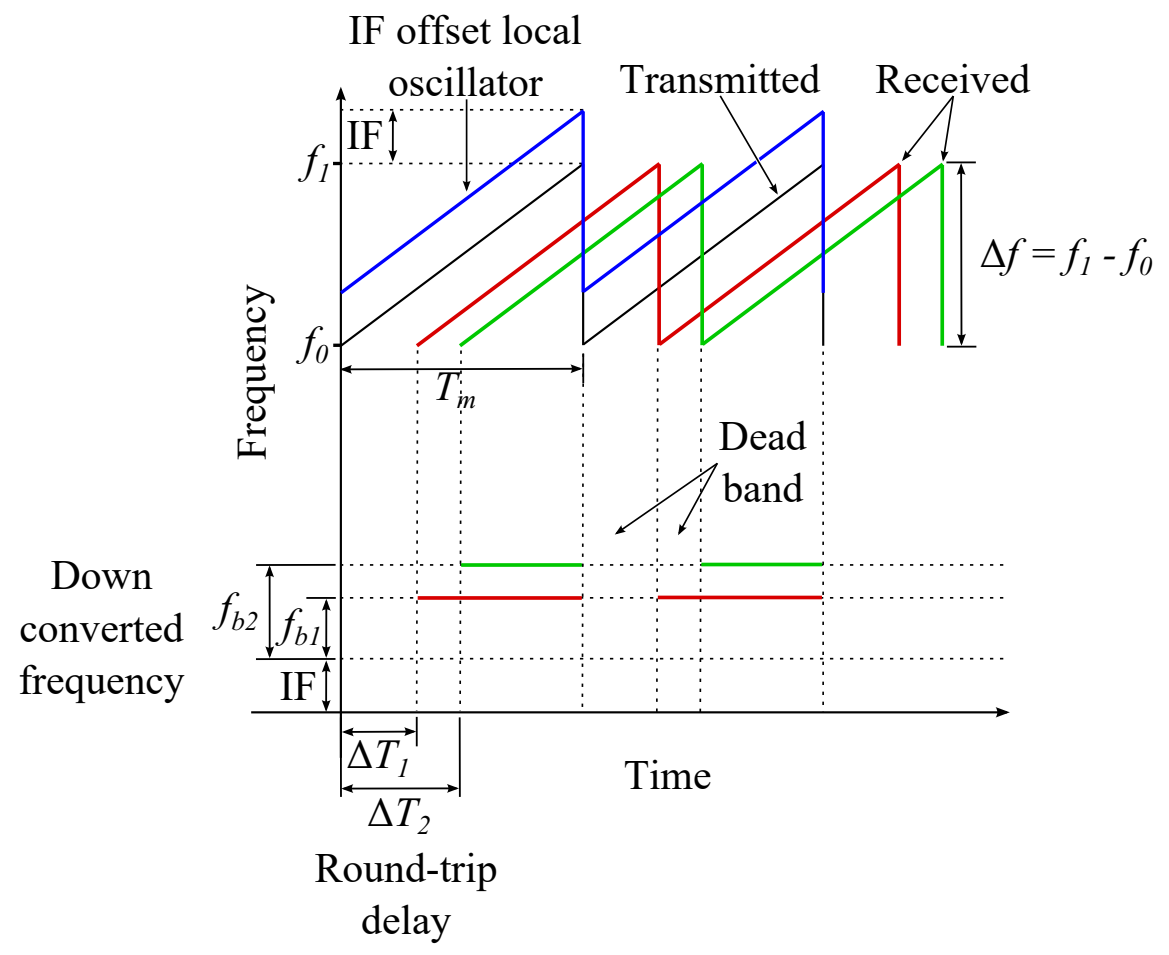

Figure 1.6: Frequency versus time plot for the IF sawtooth FM scheme, where the IF offset LO, transmitted and received signals are shown. Two reflections are depicted, with their beat frequencies $f_{b 1}$ and $f_{b 2}$ respectively, where the red line represents the target nearest to the radar.

An IF FMCW radar receiver is realised by introducing a frequency offset to the sawtooth FM scheme. The frequency offset is added to the FMCW radar receiver's LO and 
results in the down converted signals having a fixed IF offset in addition to a beat frequency, as shown in Fig. 1.6. Therefore, as illustrated in Fig. 1.1, the IF receiver's down converted spectrum has a non-zero carrier frequency and the same bandwidth as an equivalent zero-IF receiver. In section 2.2 the specifications for the FMCW radar's sawtooth FM scheme is presented.

\subsection{Project overview}

This thesis consists of three main parts: the design of an FMCW radar with an IF receiver, a study on the impact component non-linearities have on FMCW radar performance, and the manufactured IF receiver's measurements. Chapter 1 gives an introduction to the study. Chapter 2 presents an overview of the system and acts as a road map to the radar design procedure discussed in Chapter 3. The receiver's noise is analysed in Chapter 4, while the performance of the baseband and IF receiver topologies are compared in Chapter 5. The manufactured IF receiver is characterised and the measured results are presented in Chapters 6 and 7. Lastly, conclusions and recommendations are given in Chapter 8 . 


\section{Chapter 2}

\section{System design overview}

In this chapter an overview of the radar system design is presented. The block diagram in Fig. 2.1 illustrates the system's structure.

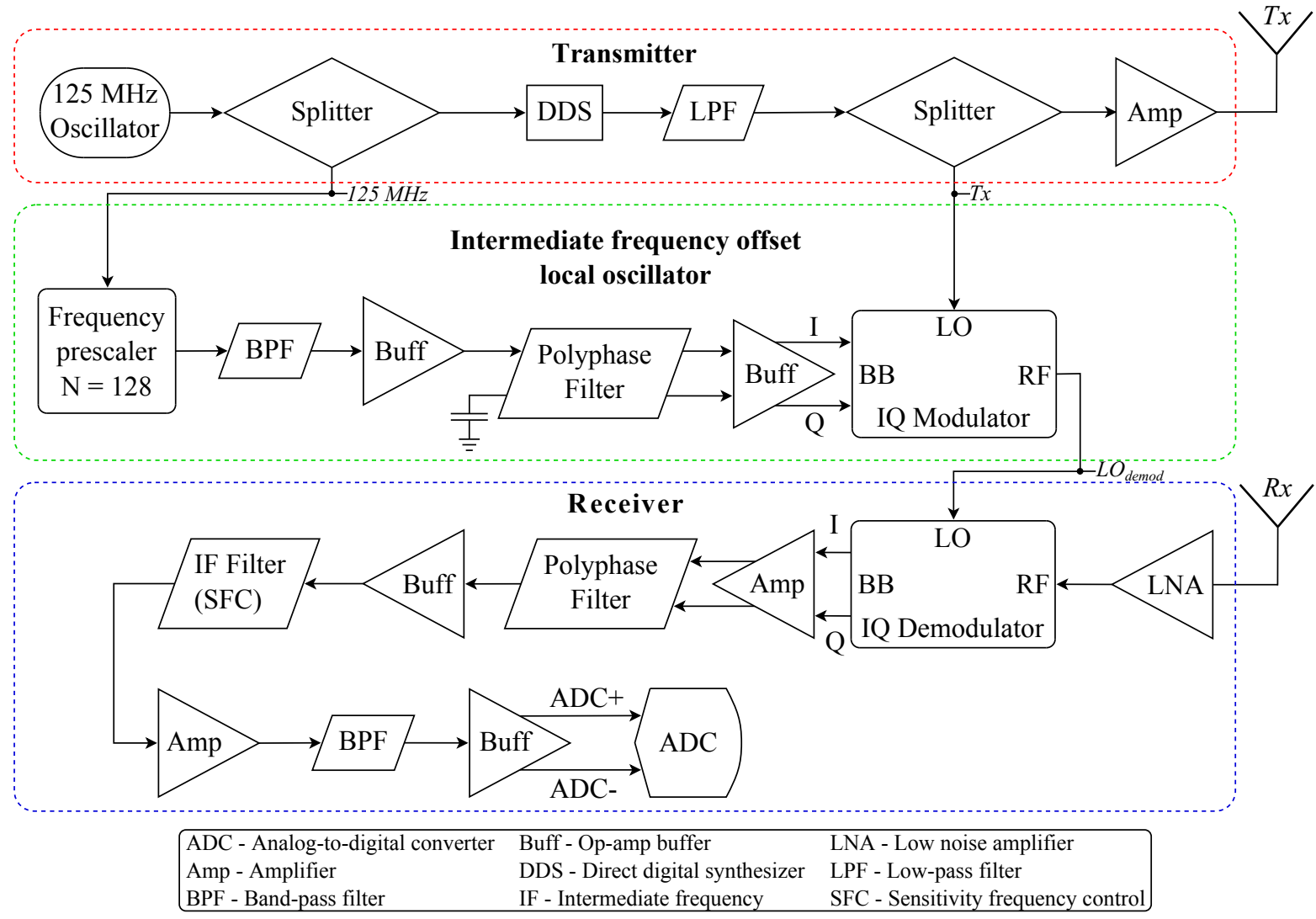

Figure 2.1: Block diagram of the IF FMCW radar.

The IF FMCW radar receiver is realised by mixing the received signal with a frequency offset version of the transmission signal in a process called heterodyning. Heterodyning results in a signal at the difference or beat frequency between the RF and LO signals. A baseband receiver, also referred to as a zero-IF receiver, applies a homodyne process that uses the transmission signal as an LO without any frequency offset, as discussed in Chapter 1. However, an IF receiver introduces a fixed frequency offset to the LO signal. Mixing the received signal with the offset LO, results in a constant frequency offset at the demodulator's baseband (BB) output, referred to as the IF. 
As stated in the study hypothesis (in section 1.2), the IF should be greater than the receiver's baseband bandwidth. The lowest order harmonic produced by a single received signal is found at twice the signal's fundamental frequency (and is known as a second order harmonic) [6]. To ensure the harmonics fall outside the receiver's passband, the IF should be at least higher than the receiver's baseband bandwidth,

$$
f_{I F}>\Delta f_{B B}
$$

However, for a practical anti-aliasing filter design the IF should be much greater than the receiver's bandwidth. Therefore, the IF is approximately an order of magnitude higher than the $100 \mathrm{kHz}$ baseband bandwidth,

$$
f_{I F}=\frac{f_{o s c}}{N}=0.977 \mathrm{MHz}
$$

where $f_{\text {osc }}$ is the $125 \mathrm{MHz}$ standard oscillator frequency, and $N$ is the pre-scaler value of 128.

\subsection{System block diagram}

The FMCW radar is broken into three sections, namely, the transmitter (Fig. 2.2), IF offset LO (Fig. 2.4), and receiver (Fig 2.12), the overall system block diagram is shown in Fig. 2.1.

\subsubsection{Transmitter}

The transmitter's operation is discussed with reference to the block diagram as presented in Fig. 2.2.

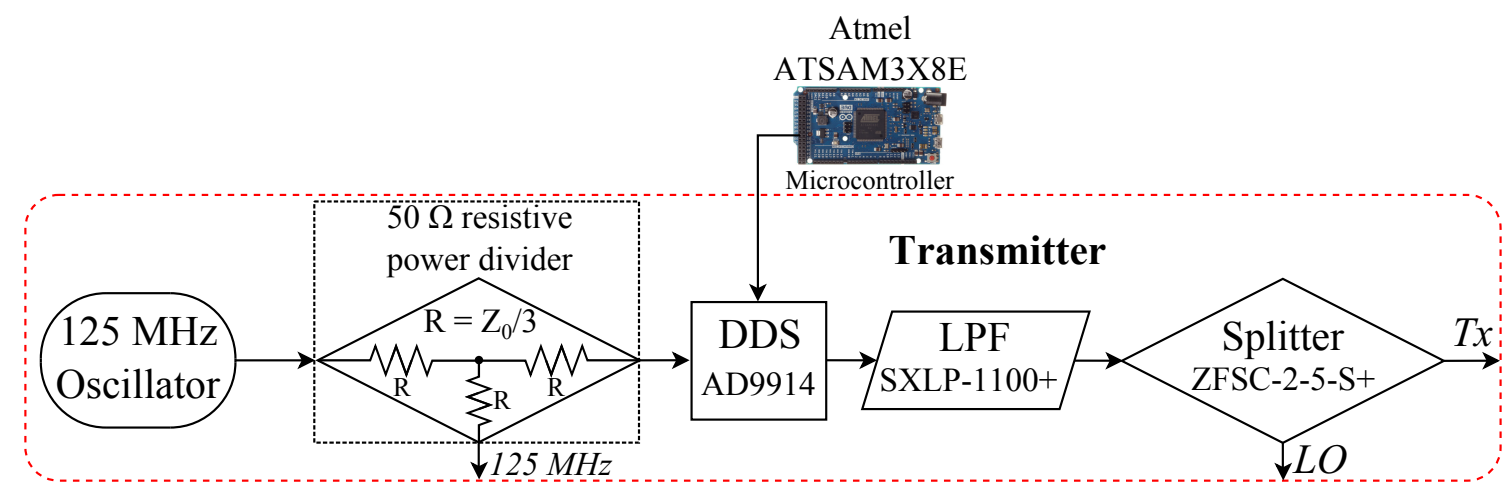

Figure 2.2: Transmitter circuit block diagram.

The first block in Fig. 2.2 is a $125 \mathrm{MHz}$ oscillator that is equally split by a three-port resistive power divider. One of the splitter outputs provide the Analog Devices AD9914 direct digital synthesiser (DDS) with an external clock signal. The phase lock loop (PLL) onboard the DDS frequency multiplies the external clock by 20 , resulting in a $2.5 \mathrm{GHz}$ internal clock $\left(f_{S, D D S}\right)$.

The DDS has a digital-to-analog converter (DAC) that outputs a sinusoidal signal sampled at $f_{S, D D S}$. Due to the sample-and-hold process, the DAC's output spectrum is affected by a sinc function, $\frac{\sin (x)}{x}$. To remove signal artefacts the output is passed through an external reconstruction filter [7]. 
The selected reconstruction filter is the Mini-Circuits SXLP-1100+ low pass filter. It has the following specification, a passband of DC to $1100 \mathrm{MHz}$ and a wide stopband of $1.44 \mathrm{GHz}$ to $8.5 \mathrm{GHz}$ with a minimum rejection of $20 \mathrm{~dB}$. The radar's modulation bandwidth spans from $400 \mathrm{MHz}$ to $800 \mathrm{MHz}$ and the unwanted harmonics generated by the DDS during the frequency modulation (FM) period are suppressed.

Lastly, the FM signal produced by the DDS is split into the transmission (Tx) and LO signals. The splitter used is the ZFSC-2-5-S+ from Mini-Circuits with a bandwidth of 10 to $1500 \mathrm{MHz}$.

\section{Direct digital synthesiser}

The DDS is controlled by an Atmel ATSAM3X8E microcontroller unit (MCU) and is set-up to periodically generate a sawtooth FM sinusoidal chirp signal that sweeps from $800 \mathrm{MHz}$ down to $400 \mathrm{MHz}$ in one millisecond. The DDS' amplitude response is measured using a spectrum analyser and the result is presented in Fig. 2.3. The amplitude response is not flat and causes FM of the IF signal. The modulation results in false targets at low beat frequencies that are suppressed by the IF filter (see Fig. 2.1). The IF filter is discussed in Chapter 3.

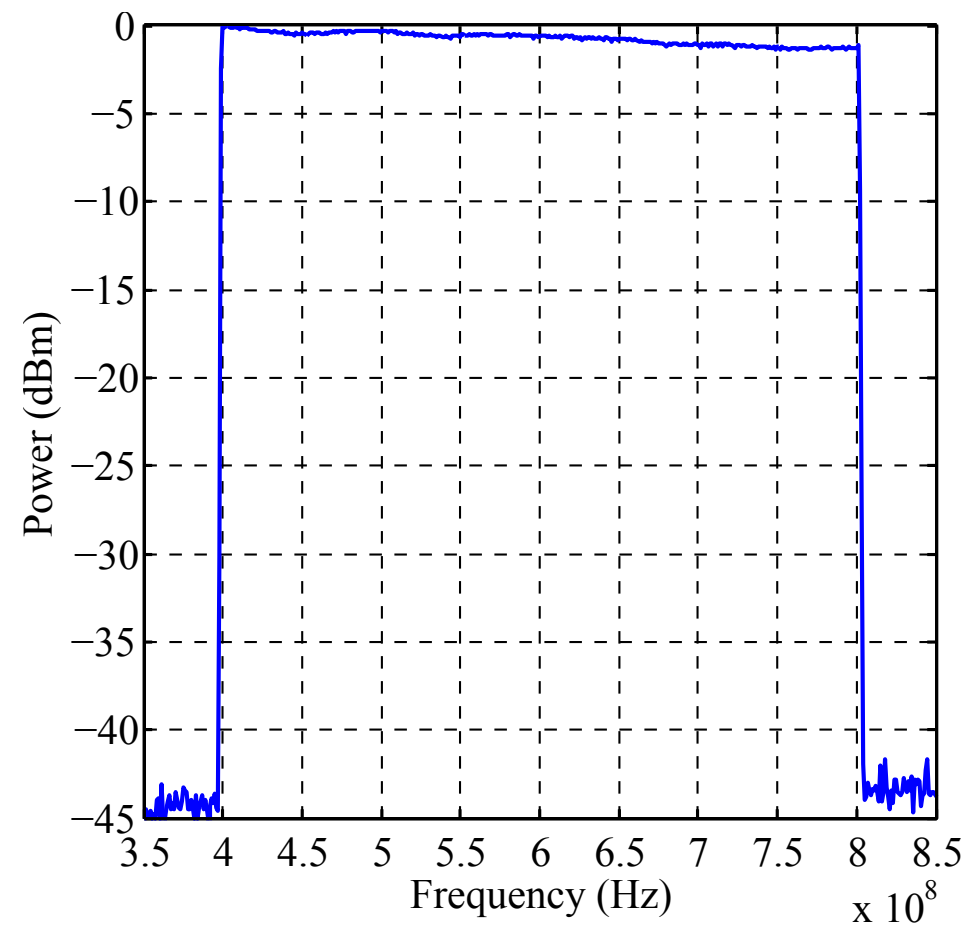

Figure 2.3: DDS amplitude response.

\subsubsection{Intermediate frequency offset local oscillator}

The receiver IF is introduced as a fixed frequency offset to the LO signal. The PPF and IQ modulator are configured to utilise signal interference to realise the frequency offset by suppressing specific spectral components. The IF offset LO circuit block diagram is provided in Fig. 2.4. 


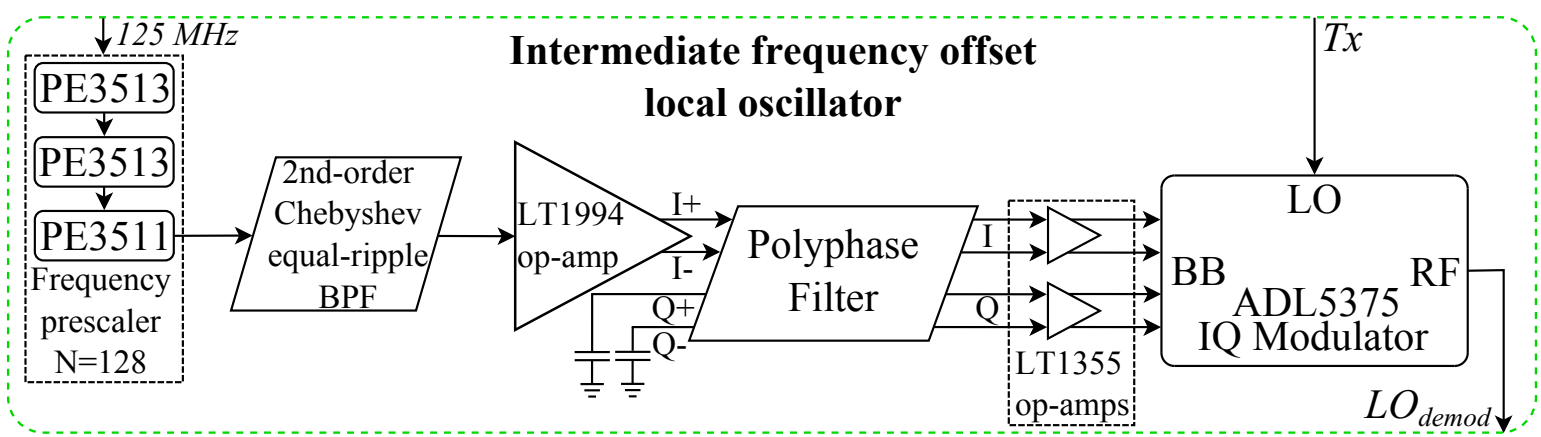

Figure 2.4: Intermediate frequency offset local oscillator circuit block diagram.

\section{Frequency prescaler}

The $125 \mathrm{MHz}$ oscillator's frequency is divided by 128 producing the IF offset signal. Three prescaler integrated circuits (ICs) from Peregrine Semiconductor are used. Two divideby-8 (PE3513) and one divide-by-2 (PE3511) ICs are cascaded producing a combined prescaler of 128 .

\section{Bandpass filter}

The prescalers are CMOS devices that work by using D-type flip-flops. The ICs have integrated pre-amps and their outputs are buffered by Schmitt-triggers. Therefore, even though the signal at the input to the prescaler is sinusoidal the output is a square wave that has many undesirable harmonics. A passive bandpass filter (BPF) is implemented to suppress these harmonics but a low pass filter (LPF) could also be used.

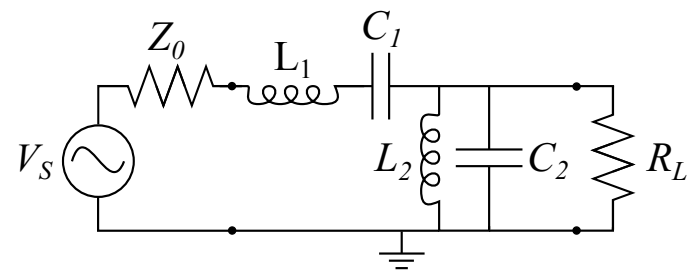

Figure 2.5: Second order equal-ripple Chebyshev bandpass filter circuit.

A second order $0.5 \mathrm{~dB}$ equal-ripple Chebyshev BPF is designed with $40 \mathrm{~dB}$ attenuation at $3 \mathrm{MHz}$, centre frequency at $f_{I F}$, and $29 \%$ fractional bandwidth. As shown in Eq. (2.1.1) the filter's fractional bandwidth is described in terms of its centre frequency $\omega_{0}$ and passband edge frequencies $\omega_{1}$ and $\omega_{2}$ respectively.

$$
\Delta=\frac{\omega_{2}-\omega_{1}}{\omega_{0}}
$$

To produce the desired filter response, a LPF prototype with a passband ripple of $0.5 \mathrm{~dB}$ is scaled in terms of impedance and frequency, and converted to baseband [8].

Table 2.1: Lumped element equations

\begin{tabular}{c||c|c|c|c}
\hline \hline Element & $L_{1}$ & $C_{1}$ & $L_{2}$ & $C_{2}$ \\
\hline Equation & $\frac{g_{1} Z_{0}}{\Delta \omega_{0}}$ & $\frac{\Delta}{g_{1} Z_{0} \omega_{0}}$ & $\frac{\Delta Z_{0}}{g_{2} \omega_{0}}$ & $\frac{g_{2}}{\Delta Z_{0} \omega_{0}}$ \\
\hline
\end{tabular}


Table 2.2: Second order equal-ripple Chebyshev bandpass filter parameters

\begin{tabular}{c|c|c|c|c|c|c}
\hline \hline $\begin{array}{c}\text { Centre } \\
\text { frequency } \\
\omega_{0}=2 \pi f_{I F}\end{array}$ & Bandwidth & \multicolumn{3}{|c|}{$\begin{array}{c}\text { Element } \\
\text { values }\end{array}$} & $\begin{array}{c}\text { Characteristic } \\
\text { impedance } \\
Z_{0}\end{array}$ & $\begin{array}{c}\text { Load } \\
\text { impedance } \\
R_{L}\end{array}$ \\
\hline $2 \pi\left(\frac{125 \mathrm{MHz}}{128}\right)$ & 0.29 & 1.4029 & 0.7071 & 1.9841 & $50 \Omega$ & $g_{3} Z_{0}$ \\
\hline
\end{tabular}

The parameters and equations required to calculate the values assigned to the circuit elements in Fig. 2.5 are provided in Tables 2.1 and 2.2 as seen in [8]. The synthesised filter's amplitude response is presented in Fig. 2.6.

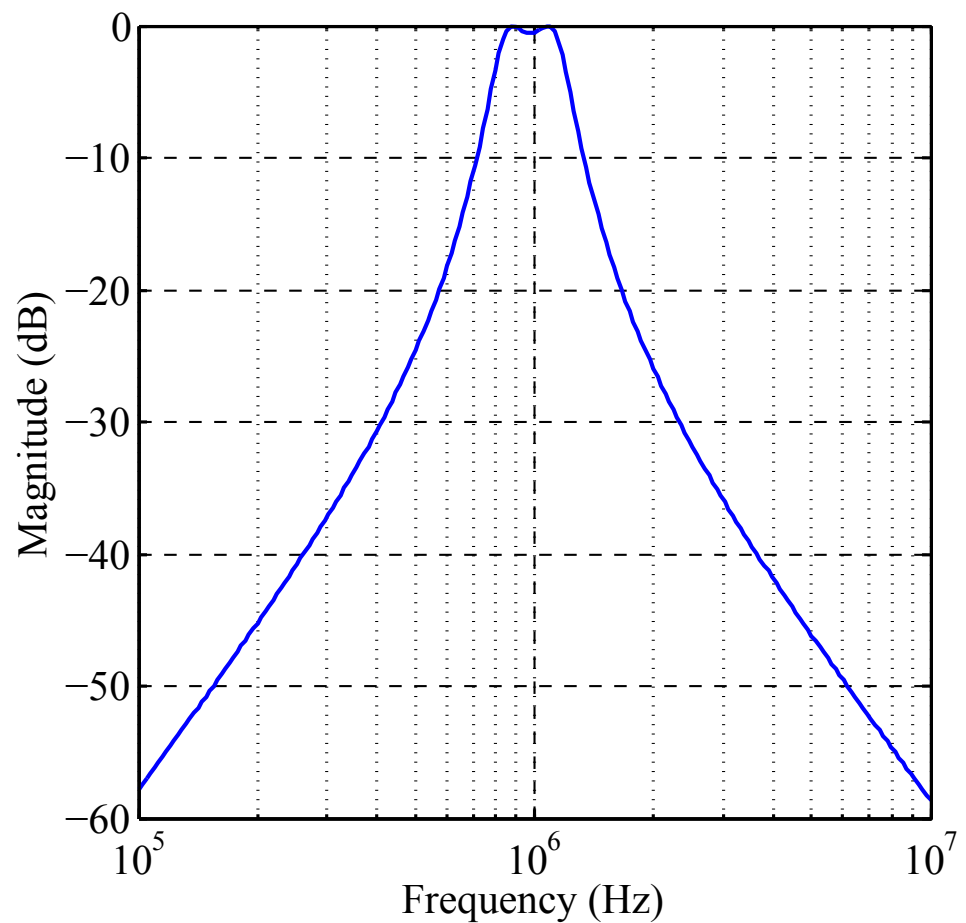

Figure 2.6: Second order equal-ripple Chebyshev bandpass filter amplitude response.

\section{Passive polyphase filter}

A commonly used polyphase splitter topology is shown in Fig. 2.7 [9]. As illustrated in Fig. 2.4, a PPF is used to split the IF signal into differential in-phase (I) and quadrature (Q) channels respectively, where the channels have $90^{\circ}$ relative phase shift.

The manner in which the input signal is applied to the PPF's first stage affects the filter's operation. In the case presented in Figs. 2.7 and 2.8, the Q-input to the first stage is signal ground $\Delta V_{Q i n, 1}=0$, and a differential signal is applied to the in-phase channel $\Delta V_{\text {Iin }, 1} \neq 0$. Exciting an ideal PPF in this way results in a constant $90^{\circ}$ phase shift between its differential outputs, $\Delta I_{\text {out }}=I_{\text {out }+}-I_{\text {out }-}$ and $\Delta Q_{\text {out }}=Q_{\text {out }+}-Q_{\text {out }}$ respectively. The PPF's I and Q output channels have equal amplitude at the centre frequency of each filter stage. Therefore, a wideband network can be realised by adding additional PPF stages. 
To illustrate the filter's operation a first order PPF presented in Fig. 2.8 (a) is analysed. The circuit is reduced to a simplified non-differential model illustrated in Fig. 2.8 (b).

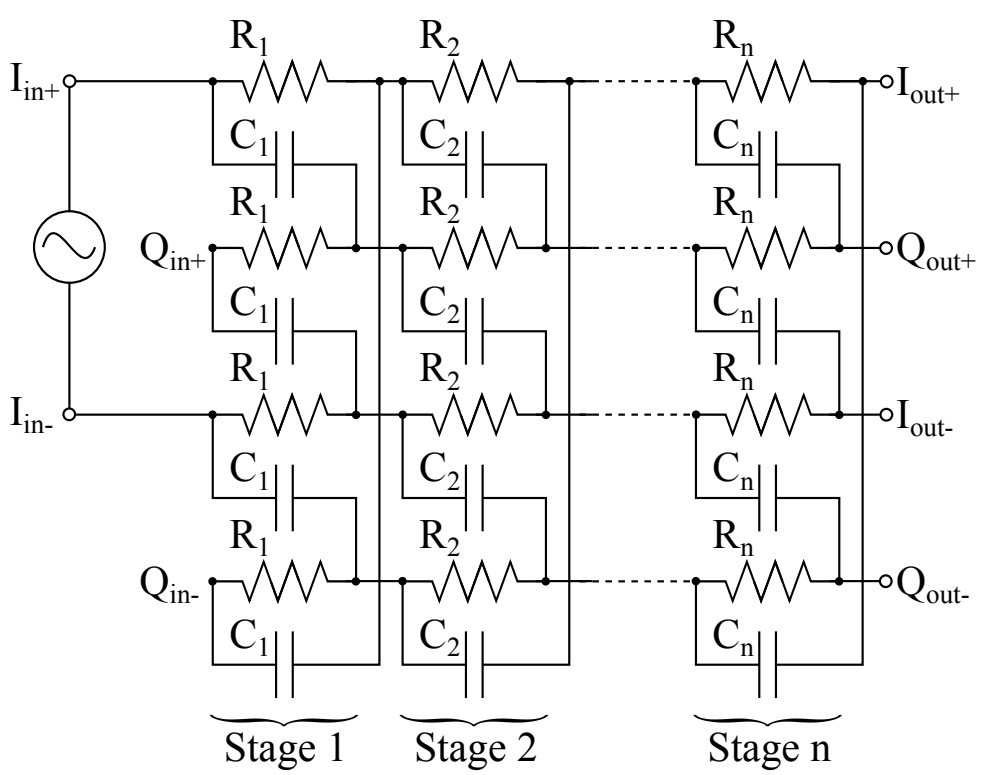

Figure 2.7: Multi-stage passive polyphase filter topology.

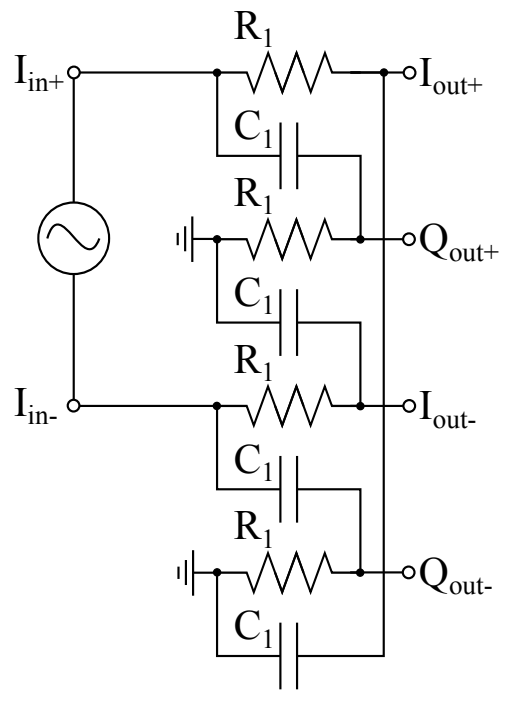

(a)

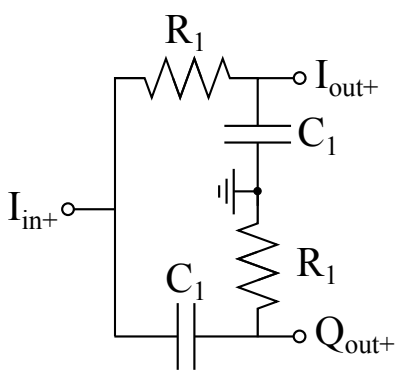

(b)

Figure 2.8: (a) First order passive polyphase filter. (b) Simplified first order passive polyphase filter.

With reference to Fig. 2.8 (b), the transfer functions from the filter's input $\left(I_{\text {in }}\right)$ to its outputs ( $I_{\text {out }}$ and $\left.Q_{\text {out }+}\right)$ are given by Eqs. (2.1.2) and (2.1.3) that resemble a LPF and high pass filter (HPF) response respectively [9].

$$
\frac{I_{\text {out }+}(j \omega)}{I_{\text {in+ }}(j \omega)}=\frac{1}{j \omega R_{1} C_{1}+1}
$$




$$
\frac{Q_{\text {out }+}(j \omega)}{I_{\text {in+ }}(j \omega)}=\frac{j \omega R_{1} C_{1}}{j \omega R_{1} C_{1}+1}
$$

The PPF's design equation is produced by setting the magnitude of Eq. (2.1.2) equal to that of Eq. (2.1.3). By solving Eq. (2.1.4) for the desired centre frequency $f_{c}=f_{I F}$ a $90^{\circ}$ phase splitter is realised.

$$
\omega_{c}=2 \pi f_{I F}=\frac{1}{R_{1} C_{1}}
$$

The first order PPF's magnitude and phase response is presented in Fig. 2.9. It is seen that the I and Q outputs have a constant $90^{\circ}$ relative phase shift and equal signal strength at the filter's centre frequency.

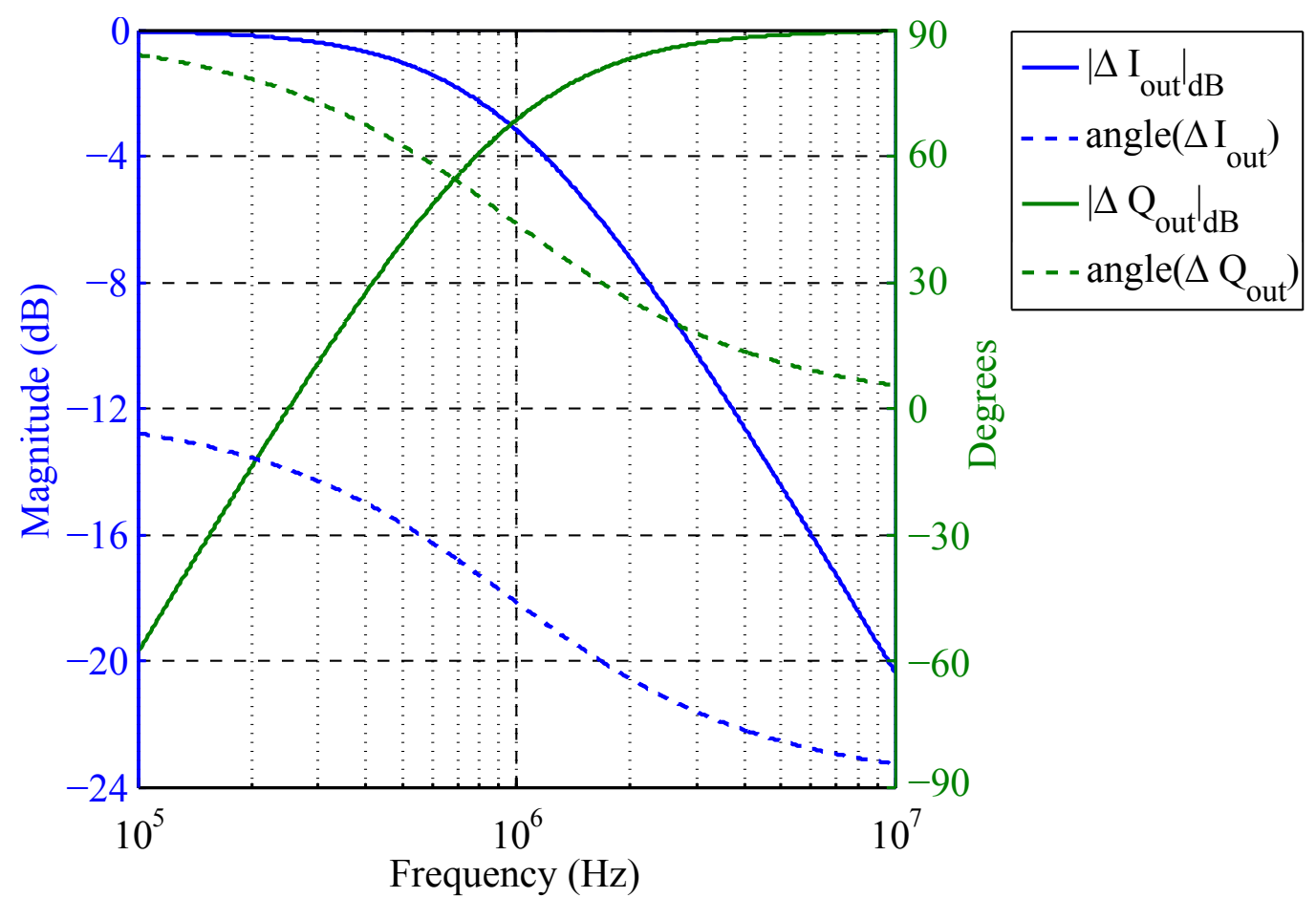

Figure 2.9: A first order passive polyphase filter's magnitude and phase response.

Using Eq. (2.1.4) the values assigned to $R_{1}$ and $C_{1}$ are selected. A small resistor value is desired to keep thermal noise as low as possible. However, to minimise the load on the buffer op-amps there is limitations on the smallest allowed resistor value.

\section{IQ modulator}

The functional operation of an IQ modulator is represented by two mixers with a quadrature phase splitter in the LO signal path, as seen in Fig. 2.10 (a). In practice the LO signal is buffered and its magnitude limit. Thus, the magnitude of the RF output is only dependent on the signal power presented to the in-phase and quadrature inputs. The device circuit model is simplified by assuming: the mixers are ideal signal multipliers and the phase splitter provides an exact $90^{\circ}$ relative phase shift. The idealised IQ modulator model is shown in Fig 2.10 (b).

The quadrature modulator is selected on the grounds of its frequency bandwidth, quadrature error and IQ amplitude balance. The chosen component is the ADL5375 
broadband quadrature modulator from Analog Devices. The device has two doublebalanced mixers and its LO quadrature generator is a wideband polyphase splitter. Consequently, the model presented in Fig. 2.10 (b) is used in calculations.

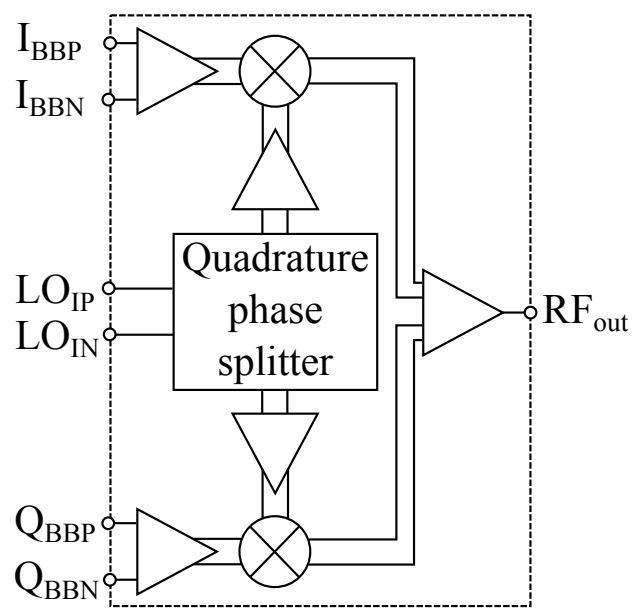

(a)

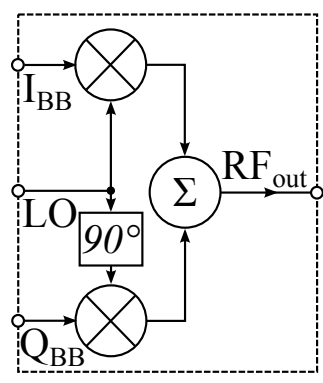

(b)

Figure 2.10: (a) IQ modulator functional block diagram. (b) Simplified IQ modulator block diagram.

\section{Intermediate frequency offset}

The IF offset is realised by using a polyphase splitter and quadrature modulator topology. Depending on the configuration of these two components the upper or lower sideband (LSB) of the resulting RF output signal is selected.

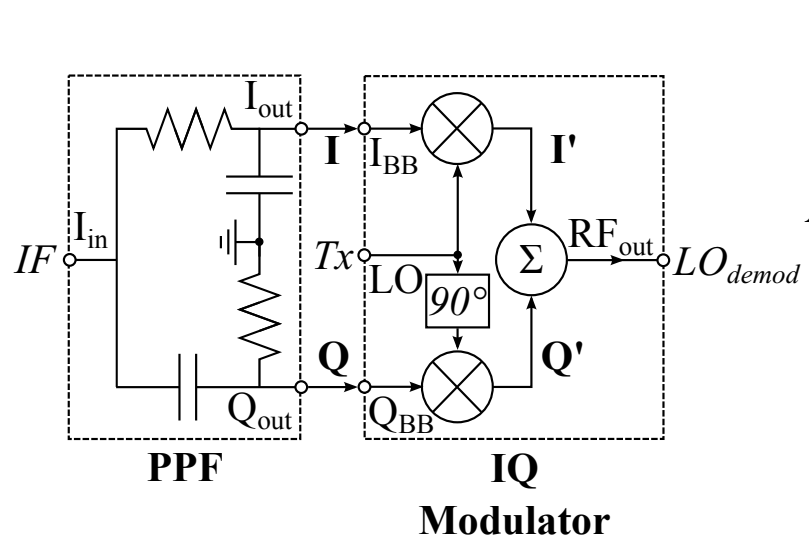

(a)

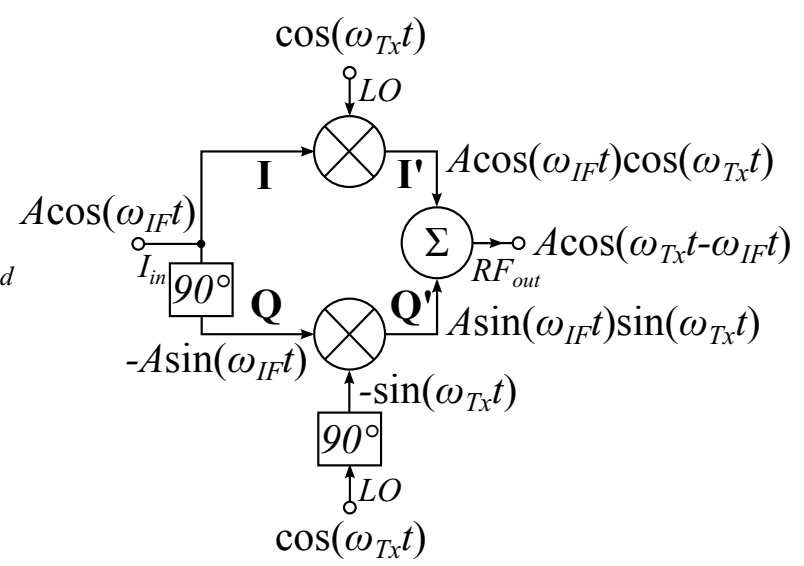

(b)

Figure 2.11: (a) Passive polyphase filter and quadrature modulator configuration. (b) Simplified mathematical representation of the modulation scheme with input IF and Tx signals resulting in an IF offset RF output signal.

It is theoretically possible to design a filter that can suppress the undesired sideband. Practically this is difficult, because the filter would have to be narrow bandwidth (less than $0.25 \%$ bandwidth) and tunable across a wide frequency range from $400 \mathrm{MHz}$ to 800 $\mathrm{MHz}$ in the frame of $1 \mathrm{~ms}$. These constraints result from the chirp signal's $400 \mathrm{MHz}$ 
bandwidth, the $1 \mathrm{~ms}$ modulation (or sweep) period, and the desired IF offset. Therefore the modulation scheme shown in Fig. 2.11 is selected. It cancels the upper sideband (USB) using destructive interference leaving the desired LSB as the IF offset LO.

The IF signal is applied to the PPF, and nodes $\mathbf{I}$ and $\mathbf{Q}$ in Fig. 2.11 are described by Eqs. (2.1.5) and (2.1.6), where $\omega_{I F}=2 \pi f_{I F}$.

$$
\begin{gathered}
I=A \cos \left(\omega_{I F} t\right) \\
Q=-A \sin \left(\omega_{I F} t\right)
\end{gathered}
$$

The radar's transmission signal is applied to the modulator's LO port. The signal then passes through an internal phase splitter. By multiplying the modulator's baseband and LO signals, as shown in Fig. 2.11 (b), results in Eqs. (2.1.7) and (2.1.8), where $\omega_{T x}$ is the transmitting chirp signal's frequency.

$$
\begin{aligned}
I^{\prime} & =A \cos \left(\omega_{T x} t\right) A \cos \left(\omega_{I F} t\right) \\
& =\frac{A}{2}\left[\cos \left(\omega_{T x} t-\omega_{I F} t\right)+\cos \left(\omega_{T x} t+\omega_{I F} t\right)\right] \\
Q^{\prime} & =A \sin \left(\omega_{T x} t\right) A \sin \left(\omega_{I F} t\right) \\
& =\frac{A}{2}\left[\cos \left(\omega_{T x} t-\omega_{I F} t\right)-\cos \left(\omega_{T x} t+\omega_{I F} t\right)\right]
\end{aligned}
$$

Finally, Eqs. (2.1.7) and (2.1.8) are summed at the RF output producing an IF offset version of the transmission signal

$$
R F_{\text {out }}=A \cos \left[\left(\omega_{T x}-\omega_{I F}\right) t\right] .
$$

Equation 2.1.9 describes the LSB. The USB is selected by swapping the I and Q channels between the PPF and IQ modulator.

\subsubsection{Receiver}

This section provides a list of low noise amplifiers (LNAs) and IQ demodulators considered for the FMCW radar receiver. The chosen LNA and IQ demodulator is selected on the grounds of its dynamic range. When the appropriate components are selected the cascaded $\mathrm{RF}$ front-end dynamic range is calculated. The following manufacturer's products are considered: Mini-Circuits, Analog Devices, Linear Technologies and Hittite.

Figure 2.12 provides an overview of the receiver chain. In Chapter 3 the receiver is discussed in detail, however, the selection of RF components are dealt with in this section.

\section{Low noise amplifier}

The LNA is selected on the grounds of its dynamic range, noise figure and absolute maximum RF input power level. The LNAs considered for the receiver's front-end are listed in Table 2.3. Each components input third order intercept point (IIP3), input 1 $\mathrm{dB}$ compression point (IP1dB), gain $(\mathrm{G})$, noise figure $(\mathrm{NF})$, spurious free dynamic range (SFDR), linear dynamic range (LDR) and absolute maximum RF input power is provided. These parameters are also discussed in Chapters 4 and 5 .

The SFDR and LDR are calculated using Eqs. (2.1.10) and (2.1.11) [6],

$$
S F D R=\frac{2}{3}\left(\mathrm{OIP} 3-N_{o}\right)
$$




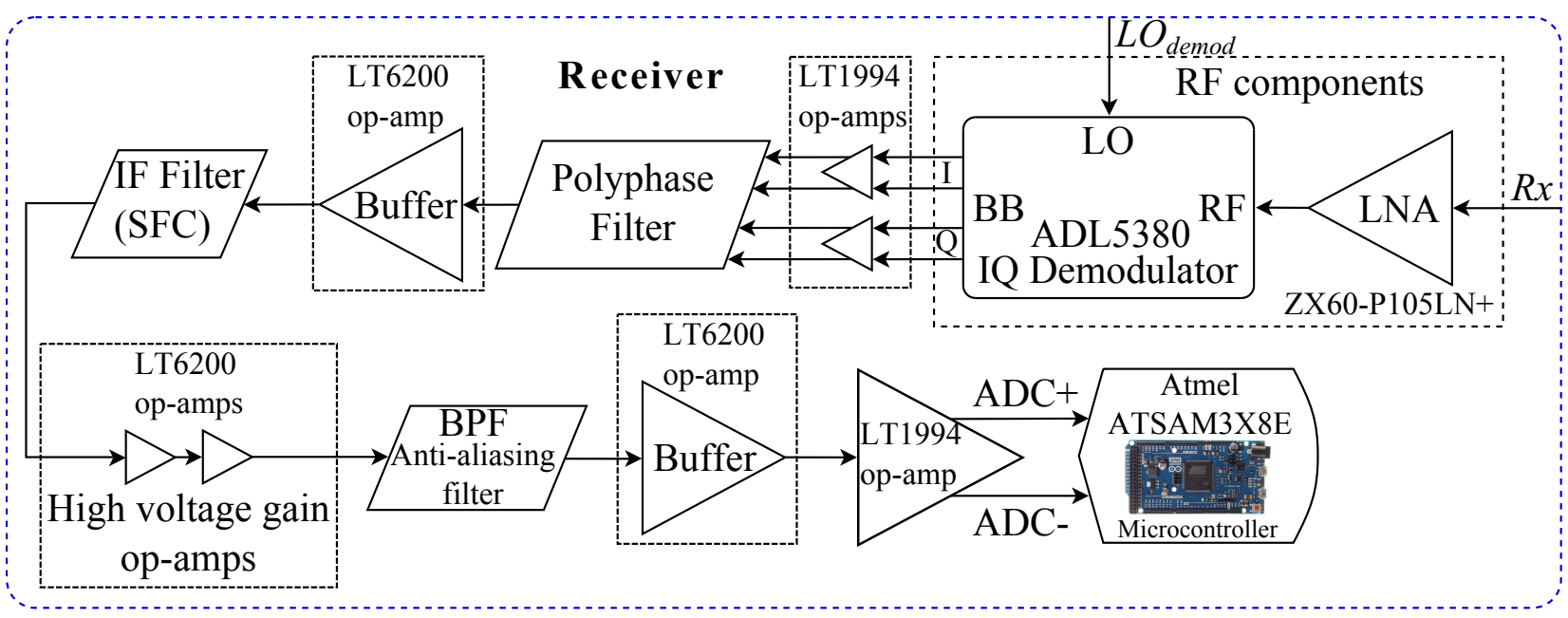

Figure 2.12: Receiver circuit block diagram.

$$
L D R=\mathrm{OP} 1 \mathrm{~dB}-N_{o},
$$

where the output third order intercept point (OIP3), output $1 \mathrm{~dB}$ compression point $(\mathrm{OP} 1 \mathrm{~dB})$ and output noise power $\left(N_{o}\right)$ are determined by Eqs. (2.1.12) to (2.1.15).

$$
\begin{gathered}
\text { OIP } 3=\operatorname{IIP} 3+G, \\
\text { OP } 1 \mathrm{~dB}=\operatorname{IP} 1 \mathrm{~dB}+G-1 . \\
N_{i}=k T_{0} \Delta f \\
N_{o}=10 \log \left(G N_{i}+k G \Delta f(F-1) T_{0}\right)
\end{gathered}
$$

All variables in Eq. (2.1.15) are linear (as derived in section 4.3), where $k$ is the Boltzmann constant, $F$ is the device's noise factor, and $\Delta f$ is the receiver's $100 \mathrm{kHz}$ baseband bandwidth.

Table 2.3: Low noise amplifier parameters as shown on the manufacturer datasheets, and the SFDR and LDR is calculated for $\Delta f=100 \mathrm{kHz}$

\begin{tabular}{c|c|c|c|c|c|c|c}
\hline \hline Part number & $\begin{array}{c}\text { IIP3 } \\
{[\mathrm{dBm}]}\end{array}$ & $\begin{array}{c}\text { IP1dB } \\
{[\mathrm{dBm}]}\end{array}$ & $\begin{array}{c}\text { Gain } \\
{[\mathrm{dB}]}\end{array}$ & $\begin{array}{c}\text { NF } \\
{[\mathrm{dB}]}\end{array}$ & $\begin{array}{c}\text { SFDR } \\
{[\mathrm{dB}]}\end{array}$ & $\begin{array}{c}\text { LDR } \\
{[\mathrm{dB}]}\end{array}$ & $\begin{array}{c}\text { Max RF } \\
{[\mathrm{dBm}]}\end{array}$ \\
\hline ADL5521 & 16.2 & 2 & 20.8 & 0.9 & 92.8 & 123.9 & 20 \\
HMC374SC70E & 20 & 4.5 & 15 & 1.6 & 94.8 & 125.7 & 15 \\
PGA-103+ & 18 & 1.5 & 21 & 0.6 & 94.2 & 123.7 & 21 \\
ZA60-P103LN+ & 16.2 & 3 & 20 & 0.7 & 92.8 & 125.1 & 21 \\
ZX60-P105LN+ & 20 & 6 & 15 & 1.9 & 94.6 & 126.9 & 23 \\
\hline
\end{tabular}

From the components listed in Table 2.3 the Mini-Circuits ZX60-P105LN+ is selected for its greater linearity and absolute maximum input power.

\section{IQ demodulator}

The demodulators are considered on the same criteria as the LNAs namely, dynamic range, noise figure and maximum $\mathrm{RF}$ input power. According to these qualifiers the Linear Technologies LT5584 demodulator is the best component. However, the Analog Devices ADL5380 was available for this project. Therefore, it is used instead. 
Table 2.4: IQ demodulator parameters as shown on the manufacturer datasheets, with $f_{L O}=900 \mathrm{MHz}, P_{L O}=0 \mathrm{dBm}$, and the SFDR and LDR is calculated for $\Delta f=100 \mathrm{kHz}$

\begin{tabular}{c|c|c|c|c|c|c|c}
\hline \hline Part number & $\begin{array}{c}\text { IIP3 } \\
{[\mathrm{dBm}]}\end{array}$ & $\begin{array}{c}\text { IP1dB } \\
{[\mathrm{dBm}]}\end{array}$ & $\begin{array}{c}\text { Gain } \\
{[\mathrm{dB}]}\end{array}$ & $\begin{array}{c}\text { NF } \\
{[\mathrm{dB}]}\end{array}$ & $\begin{array}{c}\text { SFDR } \\
{[\mathrm{dB}]}\end{array}$ & $\begin{array}{c}\text { LDR } \\
{[\mathrm{dB}]}\end{array}$ & $\begin{array}{c}\text { Max RF }_{\text {in }} \\
{[\mathrm{dBm}]}\end{array}$ \\
\hline ADL5380 & 30 & 11.6 & 7 & 13.1 & 93.8 & 121.3 & 15 \\
ADL5387 & 31 & 13 & 4.3 & 14.7 & 93.4 & 121.1 & 15 \\
HMC597LP4 & 25 & 12 & -3.5 & 15 & 89.2 & 119.8 & 15 \\
LTC5517 & 21 & 10 & 3.3 & 12.4 & 88.3 & 120.4 & 10 \\
LT5584 & 31 & 13.1 & 5.4 & 9.9 & 96.6 & 126 & 20 \\
\hline
\end{tabular}

\section{Radio frequency front-end cascaded dynamic range}

The LNA (ZX60-P105LN+) and IQ demodulator (ADL5380) constitute the RF front-end. The RF front-end's cascaded dynamic range is calculated using the parameters in Tables 2.3 and 2.4 .

With reference to Fig. 2.13, the cascaded conversion gain $\left(G_{c a s}\right)$ is the sum of the individual component's power gain

$$
G_{c a s}=G_{1}+G_{2}
$$

where $G_{1}$, OIP3' and $\mathrm{OP}^{\prime} \mathrm{dB}^{\prime}$ are the first stage power gain, third-order intercept point and output $1 \mathrm{~dB}$ compression point respectively, and $G_{2}$, OIP 3 " and OP1dB" are the corresponding values for the second stage.

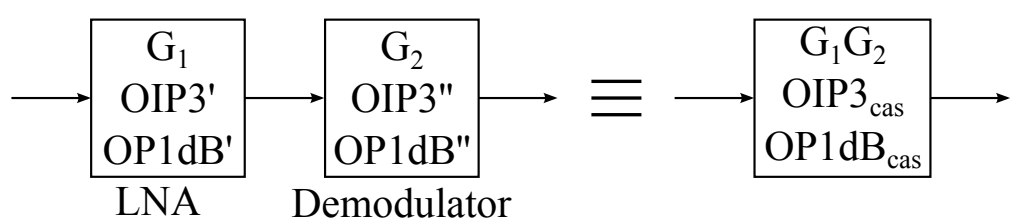

(ZX60-P105LN+) (ADL5380)

(a)

(b)

Figure 2.13: Third-order intercept point for a cascaded system: (a) two component cascaded network, (b) equivalent network.

The worst-case cascaded non-linearity results from coherent intermodulation products (IMPs) that are phase related and experience positive interference. However, it is possible that IMPs from different stages could have random relative phase. Therefore, phase delays within the stages could cause partial cancellation of IMPs as is the case with uncorrelated noise powers [8].

Assuming the worst case with coherent IMPs the cascaded system's third order intercept point is described by Eq. (2.1.17).

$$
\mathrm{OIP} 3_{c a s}=\left(\frac{1}{G_{2} \mathrm{OIP} 3^{\prime}}+\frac{1}{\mathrm{OIP}^{\prime \prime}}\right)^{-1}
$$

In the same way the cascaded output $1 \mathrm{~dB}$ compression is determined as follows

$$
\mathrm{OP} 1 \mathrm{~dB}_{c a s}=\left(\frac{1}{G_{2} \mathrm{OP} 1 \mathrm{~dB}^{\prime}}+\frac{1}{\mathrm{OP} 1 \mathrm{~dB} 3^{\prime \prime}}\right)^{-1}
$$


Table 2.5: Cascaded network parameters

\begin{tabular}{c|c|c|c|c|c|c}
\hline \hline $\begin{array}{c}\text { IIP3 } \\
{[\mathrm{dBm}]}\end{array}$ & $\begin{array}{c}\text { IP1dB } \\
{[\mathrm{dBm}]}\end{array}$ & $\begin{array}{c}\text { Gain } \\
{[\mathrm{dB}]}\end{array}$ & $\begin{array}{c}\text { NF } \\
{[\mathrm{dB}]}\end{array}$ & $\begin{array}{c}\text { SFDR } \\
{[\mathrm{dB}]}\end{array}$ & $\begin{array}{c}\text { LDR } \\
{[\mathrm{dB}]}\end{array}$ & $\begin{array}{c}\text { Max RF }_{\text {in }} \\
{[\mathrm{dBm}]}\end{array}$ \\
\hline 13.8 & -3.9 & 22 & 3.4 & 89.5 & 115.6 & 13.6 \\
\hline
\end{tabular}

Equations (2.1.17), (2.1.18), and (2.1.10) through (2.1.13) are used to generate the results provided in Table 2.5. The cascaded noise figure is calculated as shown in section 4.3.1.

By comparing the results from Tables 2.3, 2.4 and 2.5 it is clear that adding an LNA to the receiver front-end improves the overall cascaded noise figure and increases the gain. However, from Eqs. (2.1.17) and (2.1.18) it seen that the receiver's dynamic range will deteriorate with each additional stage. In Chapter 5 it is shown that the analog-to-digital converter (ADC) truncates the receiver's front-end dynamic range.

\subsection{Sawtooth frequency modulation}

The FMCW radar makes use of a sawtooth modulation scheme and any image signals received by the radar is suppressed by an image rejecting receiver topology. To ensure that the correct sideband is rejected, consider the following receiver parameters: ADC sample frequency, frequency chirp/sweep direction, and the IF offset. In section 3.2 the image rejecting receiver topology is discussed in further detail.

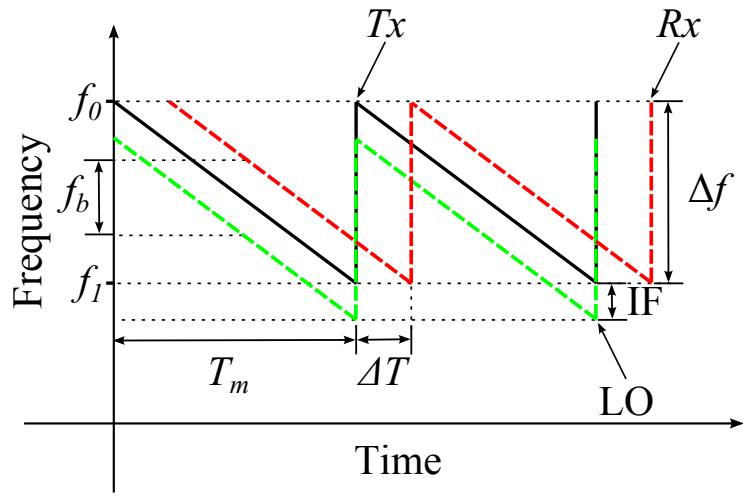

Figure 2.14: Tx (black line): sawtooth down chirp, generated by the DDS for transmission. LO (green line): transmission signal with a negative IF offset. Rx (red line): received signal.

The receiver is band limited and signals that pass are undersampled by the ADC. Undersampling occurs because the IF is higher than half the ADC's sample frequency $\left(f_{s}\right)$ and according to Nyquist's sample theory the signals are aliased,

$$
\frac{f_{s}}{2}<f_{I F}
$$

As shown in Chapter 3, received signals are undersampled and alias/wrap-down from the third Nyquist zone to the ADC's baseband frequency. Signals discretized from odd order Nyquist zones alias without being mirrored. The receiver is band limited to prevent aliasing into any of the neighbouring Nyquist zones. 
The modulated signal's frequency $\left(f_{T x}\right)$ is either swept up or down,

$$
f_{0}<f_{1}, \quad \text { or } \quad f_{1}<f_{0},
$$

and the LO can be produced by adding or subtracting the IF to or from the modulated signal's frequency,

$$
f_{L O}=f_{T x} \pm f_{I F} .
$$

As shown in Fig. 2.14, the DDS is set-up to generate a sawtooth chirp signal that sweeps from high to low, where $f_{0}=800 \mathrm{MHz}$ and $f_{1}=400 \mathrm{MHz}$. The radar is designed to operate in the ultra high frequency (UHF) band to be used as a hand held surface penetrating radar. At this operating frequency the radar's antennas can be compact, and a useful signal penetration depth and range resolution is expected. The demodulator's LO signal is produced by subtracting the IF from the transmitted signal as follows (see section 2.1.2),

$$
f_{L O}=f_{T x}-f_{I F} .
$$

By selecting a negative IF offset, using a frequency modulated down chirp, and undersampling in a non-inverting Nyquist zone, an increase in beat frequency $\left(f_{b}\right)$ is related to an increase in propagation delay $(\Delta T)$ i.e.

$$
f_{b} \propto \Delta T
$$

In other words, outside the modulation dead-band, the received signal frequency is always greater than the LO frequency even at zero propagation delay,

$$
f_{b}=f_{R x}-f_{L O}>0 .
$$

\subsection{Conclusion}

This chapter provides an overview of the FMCW radar system design. The radar's operation is broken into three main sections, namely the transmitter, IF offset LO, and receiver.

In the transmitter section, it is shown how the FM is achieved using a DDS. The method applied to realise the IF offset LO circuit is explained, and some filter designs are presented. The RF front-end components are selected, and the cascaded front-end's performance is calculated.

The sawtooth modulation scheme and the impact the IF offset, chirp direction, and ADC sampling has on the system's operation is discussed. The design decisions made in this chapter are applied throughout the study. 


\section{Chapter 3}

\section{Receiver design}

In general $\mathrm{RF}$ receivers are designed to recover a desired signal from a wide frequency spectrum that contains spurious transmitting sources, interference, and noise.

This chapter examines the FMCW radar receiver. The use of an IF receiver is discussed and a design is presented and implemented. When designing an RF receiver it is important to keep the following parameters in mind:

- Selectivity - the ability of the radar to receive desired signals while rejecting adjacent channels, image frequencies, and interference.

- Sensitivity - the minimum magnitude required from an input signal to successfully produce a detectable output signal.

- Down-conversion - the process of converting the received RF signal to a lower IF for processing.

- Isolation - an indication of the transmitters influence on the receiver.

- Gain - required to detect low power signals while minimizing the receiver chain's noise figure.

In this chapter, commonly used receiver architectures are discussed with emphasis on their advantages and disadvantages. Variations on these architectures are shown and their principal benefits explained. Image rejecting architectures are investigated and measurements presented for an implemented design. Sample theory and signal discretization is discussed in the context of the anti-aliasing filter and sensitivity frequency control (SFC) designs.

\subsection{Intermediate frequency receiver}

For FMCW radar systems, the IF is the spectral location of the carrier signal as an intermediary step during the transmission or reception process. The IF is generated by mixing the carrier signal with an LO in a process called heterodyning resulting in a signal at the difference (or beat) frequency of the two mixed signal [8].

In general IF receiver architectures are used because signal processing circuits are difficult to design and implement at high operating frequency.

IF receivers with tunable LOs are versatile and, depending on the application, capable of down-converting target frequencies to a common frequency band or several different 
frequency bands. A zero-IF receiver with the same amount of tunability requires tunable amplifiers, filters, and detectors.

Superheterodyne receivers are tuned to different frequencies by adjusting the LO's frequency at the input stage. Therefore, processing can be done after down-conversion at a single fixed IF. Without the use of an IF receiver the filter, amplifier and detector components would have to be tuned in unison every time a different frequency is selected for reception. This was the case for early tuned RF receivers [8].

\subsubsection{Zero intermediate frequency receiver}

A typical receiver architecture is the zero-IF receiver, it is also known as the homodyne or direct-conversion receiver. This architecture, as presented in Fig. 3.1, is implemented by setting the receiver's LO to the same frequency as the transmitted signal. Consequently the received RF signal is converted to baseband [8].

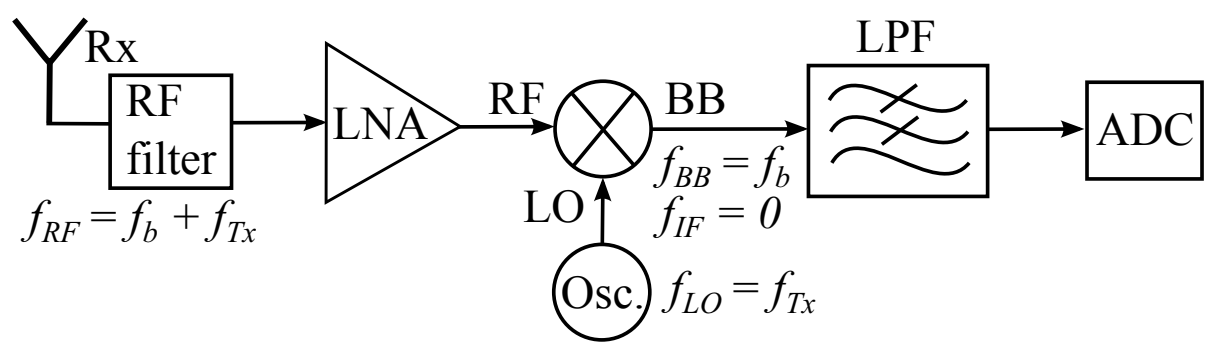

Figure 3.1: Block diagram of a zero-IF receiver topology.

\subsubsection{Intermediate frequency receiver}

A frequency offset is added to the FMCW radar receiver's LO, resulting in a fixed IF offset in addition to the beat frequency. Stated differently, the down converted received signal has a fixed bandwidth but its carrier frequency is non-zero [8]. In this study the addition of an IF offset is discussed.

In general IF receivers are less susceptible to the problem of LO-to-RF crosstalk. This issue occurs when the LO conducts or radiates through an unintended path to the mixer's $\mathrm{RF}$ input port, effectively mixing with itself and producing an unwanted DC component at the mixer's output. Conversely, if a strong in-band interference signal at the RF port has a path to the mixer's LO-input, it results in self-mixing. Numerous mechanisms contribute to poor isolation, these include substrate coupling, ground bounce, bond wire radiation, and capacitive and magnetic coupling [10]. In the case of an IF receiver the unwanted DC component is outside the frequency band of interest and is removed by filtering.

As discussed in section 3.2, image signals are a fundamental problem in IF receivers and it is solved by implementing some form of image rejection. Phase matching between the image rejecting receiver topology's in-phase and quadrature channels is critical because phase error results in less image suppression.

\subsubsection{Super-heterodyne receiver}

The super-heterodyne receiver is usually configured with two down-conversion stages, i.e. the $\mathrm{RF}$ received signal is first demodulated to an IF and then converted down to a second 
IF or baseband. In many receiver designs, where the first IF stage is at a low enough frequency the second stage is replaced by an undersampling ADC. Undersampling is a form of signal down-conversion and is discussed in section 3.3.3.

The super-heterodyne architecture is applied in many receivers due to the availability of low cost, low power, narrow band RF and IF components. The architecture potentially has good selectivity, because the desired frequency band is usually relatively narrow compared to the center frequency. The architecture is also immune to most DC problems that affect homodyne architectures [8].

\subsection{Image signals and image rejection}

Frequency down-conversion is an essential operation in RF receivers that translates the incoming RF signal to an IF. This is typically performed by mixing the RF signal with the LO [11]. In practice mixers generate many frequency components, where the component of interest is found by the difference in frequency between the RF and LO signals

$$
f_{I F}=\left|f_{R F}-f_{L O}\right| .
$$

Given that a double-sideband (DSB) signal is received, two RF signals can satisfy Eq. $(3.2 .1)$

$$
\begin{aligned}
& f_{R F, \text { upper }}=f_{L O}+f_{I F}, \\
& f_{R F, \text { lower }}=f_{L O}-f_{I F},
\end{aligned}
$$

where Eqs. (3.2.2) and (3.2.3) represent the upper and lower sideband frequencies respectively. Therefore, the classic super-heterodyne receiver topology does not discriminate between the upper and lower sidebands, and both components are mixed down to the same IF.

As discussed in section 2.2 either the upper or lower sideband is designed to carry the target reflections. For this study the upper sideband is selected; therefore, the lower sideband has the potential to carry unwanted signals at image frequencies

$$
f_{\text {image }}=f_{R F, \text { lower }}
$$

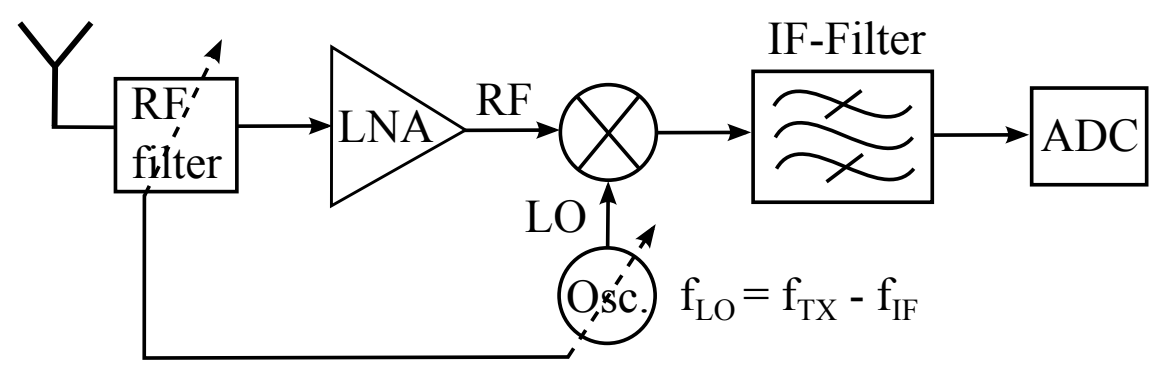

Figure 3.2: Schematic representation of the IF receiver topology.

In many super-heterodyne topologies, image suppression is done before down-conversion by a narrow band high quality factor image rejection filter. To allow for a practical filter design, a high IF is desired so that the RF signal is relatively far from the image frequency $f_{R F, \text { upper }}>>f_{\text {image }}[12]$.

It is challenging to implement the image rejecting super-heterodyne topology for a FMCW radar receiver, because the RF and LO signals are frequency modulated. Thus 
the image rejecting filter's centre frequency has to sweep alongside the chirp to effectively remove the unwanted image signals without suppressing the desired signals. The classic IF receiver topology is shown in Figure 3.2.

To understand the problem of image signals, consider the process of down-conversion as represented in Figs. 3.3 and 3.4. Mixing the desired RF signals with an LO shifts the carrier to an IF. If $X(\omega)$ represents the spectrum of a real signal $x(t)$, its spectrum will adhere to

$$
|X(-\omega)|=|X(\omega)|
$$

Real signals consist of an equal contribution of positive and negative frequency components. The spectrum at $f_{L O}-f_{I F}$ is translated to the same IF as the signals at $f_{L O}+f_{I F}$. In this case, the signals at frequency $f_{L O}-f_{I F}$ is the image frequency band. If no image rejection is implemented, undesired signals in the image band will translate to the same IF band as the desired RF signals. This leads to signal distortion and results in spurious targets in the IF band.
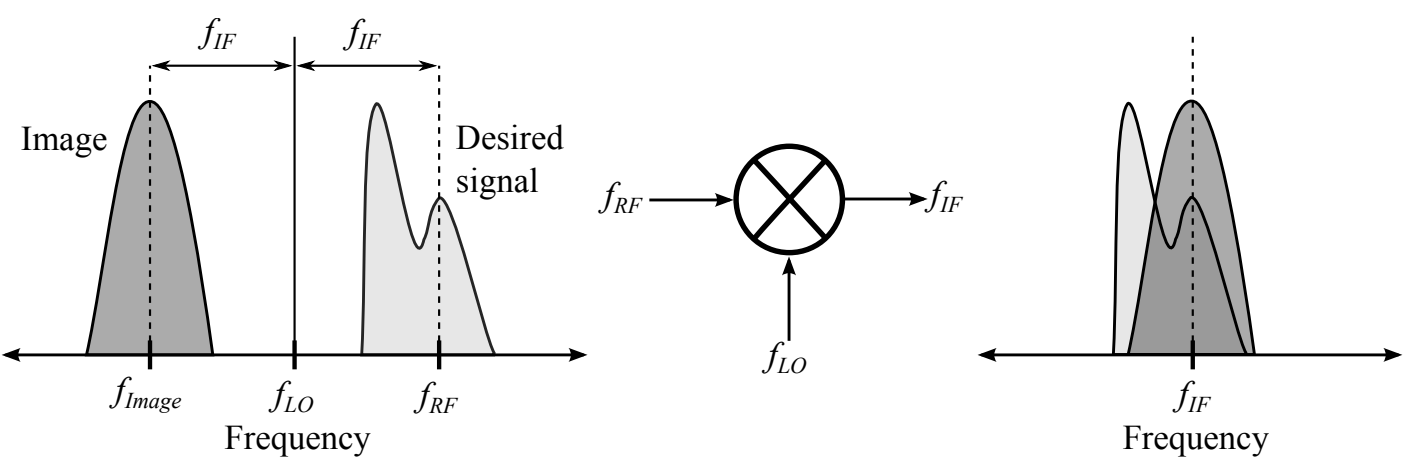

Figure 3.3: Down conversion without image-rejection filter.
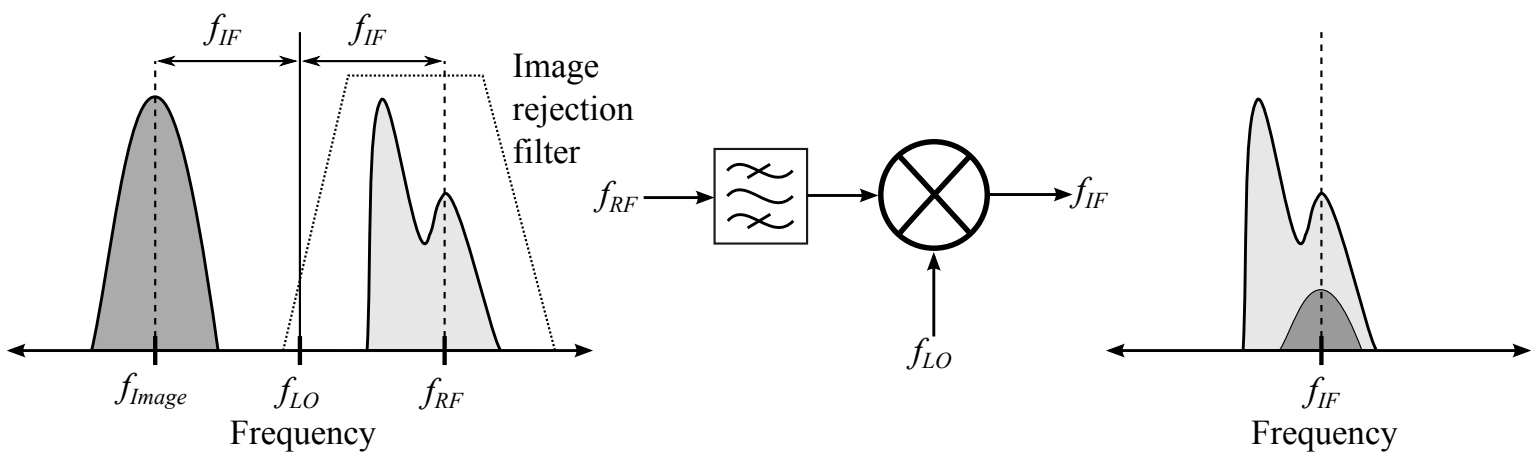

Figure 3.4: Down conversion with image-rejection filter.

The image rejection performance metric for $\mathrm{RF}$ receivers is known as the image rejection ratio (IRR). It is defined as the ratio between the magnitude in the attenuation band to that in the passband [11],

$$
I R R=\frac{\text { Desired Signal Level }}{\text { Image Signal Level }}
$$

The IRR required to ensure the desired signal's integrity, varies with application. For an FMCW radar receiver, all image signals should be suppressed sufficiently so that they are 
not detected as false targets by the ADC. The image signal power should be below the ADC's minimum detectable signal level.

Image suppression is also applied to the noise in the rejected sideband. Over a narrow band, noise is described as a signal at a single frequency that has randomly varying amplitude and phase. Therefore, by rejecting the image sideband, the receiver's sensitivity is improved.

\subsubsection{Image rejection architectures}

This section is concerned with image rejecting receiver architectures and focuses on the use of mixers and polyphase filters to obtain the required rejection.

For a PPF design, signal path symmetry and component parasitic effects should be taken into account [11]. However, at low frequencies these variables do not have such a great influence on the overall performance of the PPF. Techniques are proposed by Haddad et al [11], dedicated to layout matching and optimum component sizing to increase the IRR.

The Hartley and Weaver image rejection architectures make use of PPFs and either a half-complex or full-complex image rejection block as seen in Figs. 3.5 and 3.6 [12].

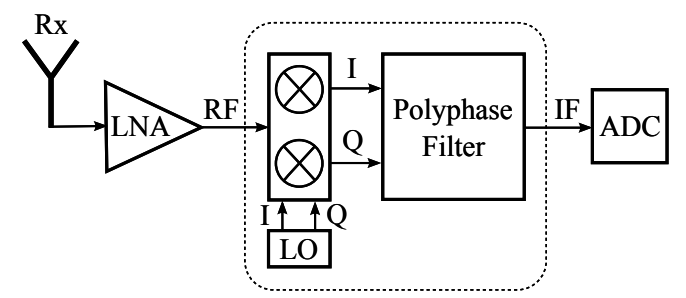

(a)

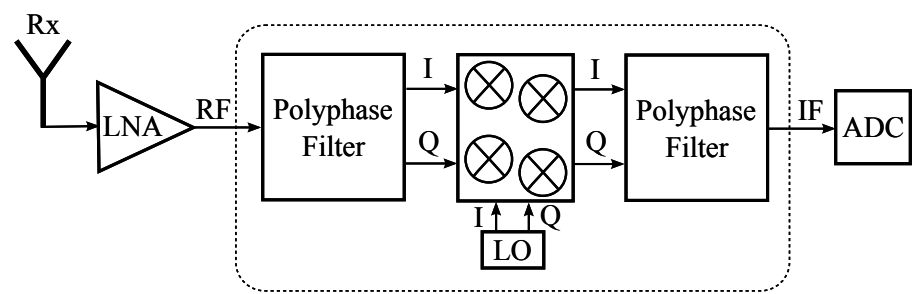

(b)

Figure 3.5: (a) Half complex and (b) full complex receiver architectures using polyphase filters.

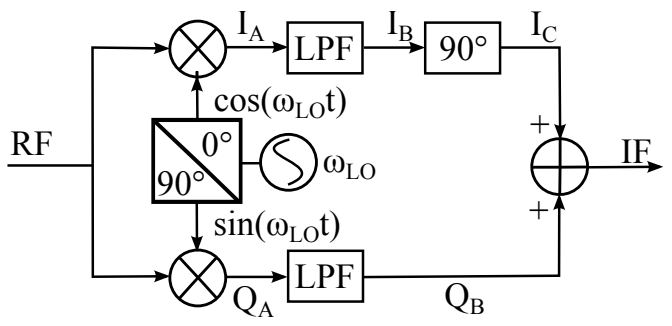

(a)

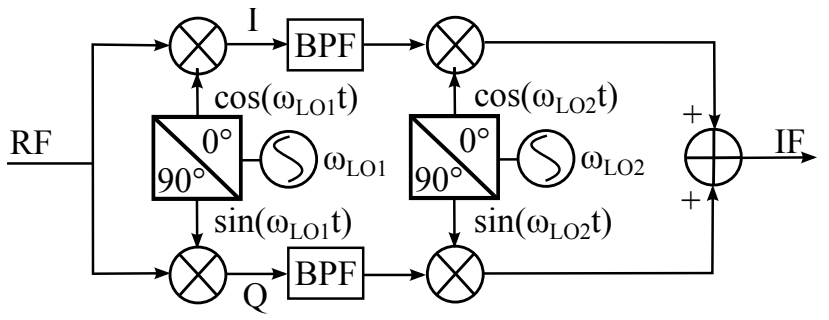

(b)

Figure 3.6: (a) Hartley (Half complex) and (b) Weaver (full complex) image rejection architectures.

The level of image rejection by the Hartley and Weaver architecture's is dependent on three parameters: the I and Q channel balance (phase and magnitude error), mixer matching and symmetry, and polyphase filter performance [11]. The principal drawback to the Hartley architecture is its sensitivity to signal path mismatches [13].

For the IF receiver design the required image rejection is narrow band compared to the carrier frequency ( $10 \%$ bandwidth). The image rejecting architecture's operating frequency is considered low with regards to layout and lumped element performance. 
The IF band is from $0.977 \mathrm{MHz}$ to $1.077 \mathrm{MHz}$, therefore a single stage PPF will provide satisfactory image rejection. Component tolerances and temperature variation have less of an impact on the IRR when fewer PPF stages are used. The number of lumped elements are directly correlated to how stringent the restrictions on component value variation are [11].

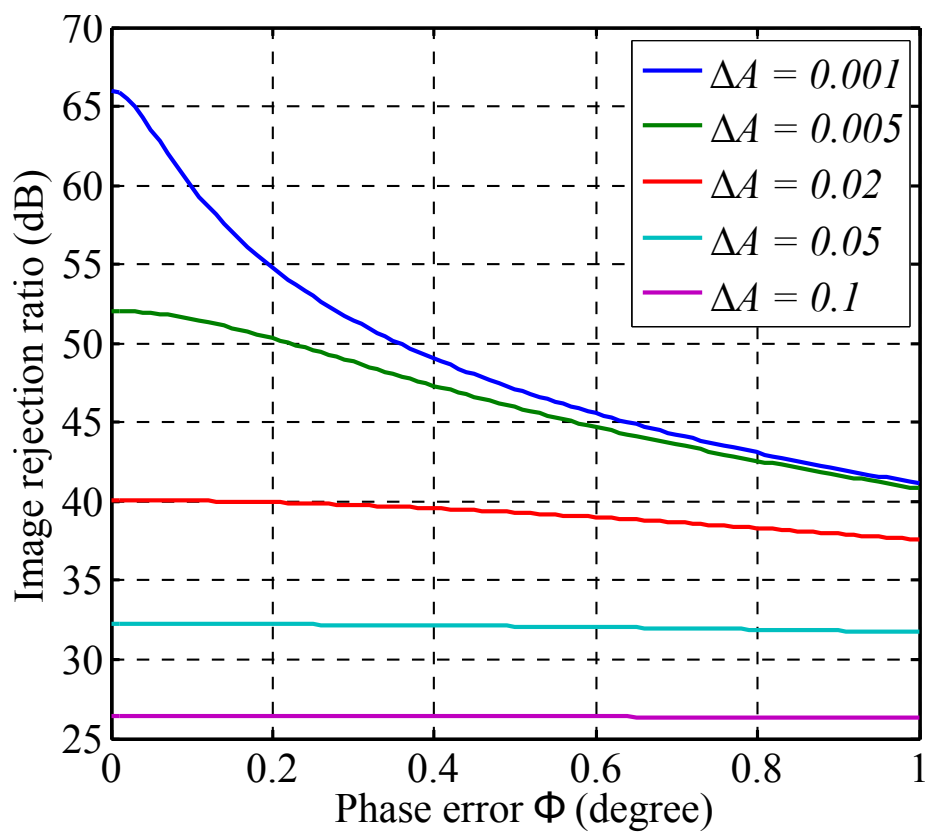

Figure 3.7: IRR versus phase error at different gain mismatch values.

Typically an IRR of around 30 to $40 \mathrm{~dB}$ is expected for the basic Hartley and Weaver implementations. Lower image rejection results from phase and gain mismatch between the I and Q channels. The IRR is expressed as a function of the mismatches as is illustrated in Fig. 3.7 and is described by

$$
\operatorname{IRR}=\frac{1+(1+\Delta A)^{2}+2(1+\Delta A) \cos (\Delta \phi)}{1+(1+\Delta A)^{2}-2(1+\Delta A) \cos (\Delta \phi)}
$$

where $\Delta A$ is the gain error and $\Delta \phi$ is the phase deviation from $90^{\circ}$ between the $\mathrm{I}$ and Q channels [14]. The ADL5380 demodulator's gain and phase mismatch is respectively, $\Delta A<0.1$ and $\Delta \phi<1^{\circ}$ at an LO frequency of $400 \mathrm{MHz}$ to $3 \mathrm{GHz}$ [15]. In the worst case the FMCW radar receiver's image rejection ratio would be around $27 \mathrm{~dB}$.

More sophisticated image rejection architecture make use of negative feedback loops to correct for gain and phase mismatch [16]. After considering the options at hand, it is decided to implement the half complex Hartley image rejection architecture because of the low complexity and the number of components required.

\subsubsection{Passive polyphase filters}

The PPF is briefly discussed in Chapter 2 for use as a phase splitter. In the case of the Hartley image rejection architecture the PPF is driven at each input by a differential signal as depicted in Fig. 3.8 (a). The manner in which a PPF is fed affects the filter's operation [9]. 
When the PPF is excited at both inputs, its operation is illustrated by calculating the output voltage at node $I_{\text {out }}$ in Fig. 3.8 (b). By applying voltage division and superposition Eq. (3.2.8) is derived.

$$
\begin{aligned}
V_{\text {Iout }+}(s) & =V_{\text {Iin }+}(s) \frac{\left(s C_{1}\right)^{-1} \| Z_{L}}{R_{1}+\left(s C_{1}\right)^{-1} \| Z_{L}} \\
& +V_{\text {Qin }+}(s) \frac{R_{1} \| Z_{L}}{\left(s C_{1}\right)^{-1}+R_{1} \| Z_{L}}
\end{aligned}
$$

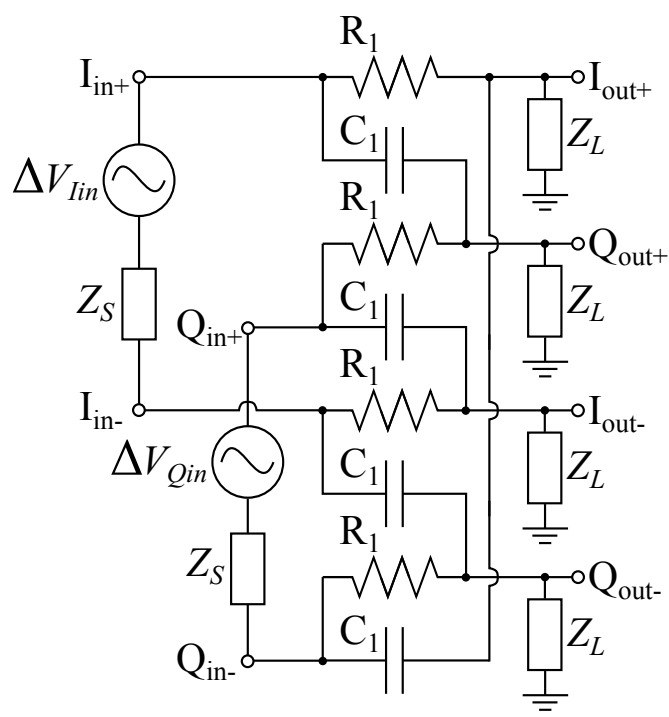

(a)

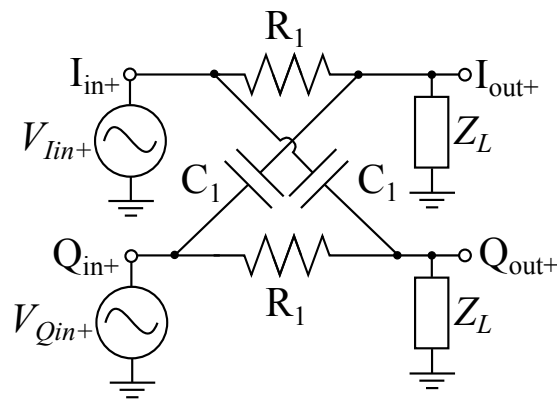

(b)

Figure 3.8: (a) First order passive polyphase filter excited differentially at both inputs. (b) Simplified first order passive polyphase filter fed at both inputs.

The practical radar system's PPFs have buffered inputs and outputs. Operational amplifiers (op-amps) are used as buffers to ensure a low source impedances $\left(Z_{S} \approx 0\right)$ and high load impedances $\left(Z_{L} \approx \infty\right)$. These approximations reduce Eq. (3.2.8) to

$$
\begin{aligned}
V_{\text {Iout }+}(s) & =V_{\text {Iin }+}(s) \frac{\left(s C_{1}\right)^{-1}}{R_{1}+\left(s C_{1}\right)^{-1}} \\
& +V_{\text {Qin+ }}(s) \frac{R_{1}}{\left(s C_{1}\right)^{-1}+R_{1}} .
\end{aligned}
$$

Equation (2.1.4) derived in Chapter 2 is used to design the PPF and provide optimal image rejection at the receiver's baseband centre frequency $\left(\omega_{c}=1.027 \mathrm{MHz}\right)$. By assuming operation at the centre frequency, Eq. (3.2.9) is reduced to the following expression

$$
\begin{aligned}
V_{\text {Iout }+}\left(j \omega_{c}\right) & =V_{\text {Iin }+}\left(j \omega_{c}\right) \frac{1}{1+j}, \\
& +V_{\text {Qin }+}\left(j \omega_{c}\right) \frac{j}{1+j} .
\end{aligned}
$$

It is clear from Eq. (3.2.10) that a relative phase shift of $90^{\circ}$ is introduced to the PPF's in-phase and quadrature inputs. 


\subsubsection{Hartley image rejection architecture analysis}

The Harley image rejection architecture is shown in Figs. 3.5 (a) and 3.6 (a). The incoming RF signal is mixed with an in-phase and quadrature LO to produce the I and $\mathrm{Q}$ channels respectively. The IQ demodulator frequency response is taken into account by including a low pass filter (LPF) on each baseband output, as depicted in Fig. 3.6 (a). The Analog Devices ADL5380 demodulator used in the receiver has a $3 \mathrm{~dB}$ bandwidth of $390 \mathrm{MHz}$. A PPF provides the $90^{\circ}$ relative phase shift required between nodes $I_{B}$ and $Q_{B}$. When the PPF produces a single sideband signal, either the upper or lower sideband is suppressed, and signals at nodes $I_{C}$ and $Q_{B}$ are summed.

To simplify the calculations it is assumed that the mixers are ideal signal multipliers. The mixer input signals are given by

$$
\begin{aligned}
& A_{R F}=\cos \left(\omega_{R F} t+\theta_{R F}\right), \\
& A_{L O_{I}}=\cos \left(\omega_{L O} t+\theta_{L O}\right), \\
& A_{L O_{Q}}=\sin \left(\omega_{L O} t+\theta_{L O}\right),
\end{aligned}
$$

where $A_{R F}$ is the incoming RF signal, and the in-phase and quadrature components of the LO signal are represented by $A_{L O_{I}}$ and $A_{L O_{Q}}$ respectively .

The signals on channels I $\left(A_{I_{A}}\right)$ and $\mathrm{Q}\left(A_{Q_{A}}\right)$ are determined by multiplying Eq. (3.2.11) with Eqs. (3.2.12) and (3.2.13),

$$
\begin{aligned}
A_{I_{A}} & =A_{R F} \times A_{L O_{I}}, \\
& =\cos \left(\omega_{R F} t+\theta_{R F}\right) \cos \left(\omega_{L O} t+\theta_{L O}\right), \\
& =\frac{1}{2}\left\{\cos \left[\left(\omega_{R F}+\omega_{L O}\right) t+\left(\theta_{R F}+\theta_{L O}\right)\right]+\cos \left[\left(\omega_{R F}-\omega_{L O}\right) t+\left(\theta_{R F}-\theta_{L O}\right)\right]\right\}, \\
A_{Q_{A}} & =A_{R F} \times A_{L O_{Q}}, \\
& =\cos \left(\omega_{R F} t+\theta_{R F}\right) \sin \left(\omega_{L O} t+\theta_{L O}\right), \\
& =\frac{1}{2}\left\{\sin \left[\left(\omega_{R F}+\omega_{L O}\right) t+\left(\theta_{R F}+\theta_{L O}\right)\right]-\sin \left[\left(\omega_{R F}-\omega_{L O}\right) t+\left(\theta_{R F}-\theta_{L O}\right)\right]\right\},
\end{aligned}
$$

where the frequency term at $\omega_{R F}-\omega_{L O}$ produces the IF.

The signals at nodes $I_{A}$ and $Q_{A}$ described by Eqs. (3.2.14) and (3.2.15) are filtered. The RF and LO frequencies are of the same order of magnitude but the IF is two orders of magnitude lower. Therefore, the summing frequency components $\left(\right.$ at $\omega_{R F}+\omega_{L O}$ ) fall outside the demodulator's $3 \mathrm{~dB}$ bandwidth. The FMCW radar's transmitted signal is modulated to sweep from $800 \mathrm{MHz}$ to $400 \mathrm{MHz}$.

After the signals are mixed and filtered, two outcomes are possible. The first case considers the upper sideband, where $\omega_{R F}>\omega_{L O}$, and the second case the lower sideband, where $\omega_{R F}<\omega_{L O}$. In both cases the IF is equal in magnitude as shown by Eq. (3.2.1). The following calculation are done with reference to Fig. 3.6 (a).

\section{Case I - upper sideband $\left(\omega_{\mathbf{R F}}>\omega_{\mathrm{LO}}\right)$}

The filtered signals at nodes $I_{B}$ and $Q_{B}$ are represented by Eqs. (3.2.16) and (3.2.17).

$$
A_{I_{B}}=\frac{1}{2} \cos \left[\left(\omega_{R F}-\omega_{L O}\right) t+\left(\theta_{R F}-\theta_{L O}\right)\right]
$$




$$
A_{Q_{B}}=-\frac{1}{2} \sin \left[\left(\omega_{R F}-\omega_{L O}\right) t+\left(\theta_{R F}-\theta_{L O}\right)\right]
$$

The in-phase channel $I_{B}$ given by Eq. (3.2.16) is phase shifted by $90^{\circ}$ resulting in Eq. (3.2.18).

$$
\begin{aligned}
A_{I_{C}} & =\frac{1}{2} \cos \left[\left(\omega_{R F}-\omega_{L O}\right) t+\left(\theta_{R F}-\theta_{L O}\right)+90^{\circ}\right] \\
& =-\frac{1}{2} \sin \left[\left(\omega_{R F}-\omega_{L O}\right) t+\left(\theta_{R F}-\theta_{L O}\right)\right]
\end{aligned}
$$

Summing the in-phase and quadrature channels described by Eqs. (3.2.17) and (3.2.18) results in case I's output signal

$$
A_{I_{C}}+A_{Q_{B}}=-\sin \left[\left(\omega_{R F}-\omega_{L O}\right) t+\left(\theta_{R F}-\theta_{L O}\right)\right] .
$$

\section{Case II - lower sideband $\left(\omega_{\mathrm{RF}}<\omega_{\mathrm{LO}}\right)$}

The procedure for case $\mathrm{I}$ is repeated in case II. The signals at nodes $I_{B}$ and $Q_{B}$ are calculated as,

$$
\begin{aligned}
A_{I_{B}} & =\frac{1}{2} \cos \left[-\left(\omega_{R F}-\omega_{L O}\right) t+\left(\theta_{R F}-\theta_{L O}\right)\right], \\
& =\frac{1}{2} \cos \left[\left(\omega_{R F}-\omega_{L O}\right) t-\left(\theta_{R F}-\theta_{L O}\right)\right], \\
A_{Q_{B}} & =-\frac{1}{2} \sin \left[-\left(\omega_{R F}-\omega_{L O}\right) t+\left(\theta_{R F}-\theta_{L O}\right)\right], \\
& =\frac{1}{2} \sin \left[\left(\omega_{R F}-\omega_{L O}\right) t-\left(\theta_{R F}-\theta_{L O}\right)\right] .
\end{aligned}
$$

The in-phase channel from Eq. (3.2.20) is phase shifted by $90^{\circ}$ producing the signal at node $I_{C}$,

$$
A_{I_{C}}=-\frac{1}{2} \sin \left[\left(\omega_{R F}-\omega_{L O}\right) t-\left(\theta_{R F}-\theta_{L O}\right)\right] .
$$

Equations (3.2.21) and (3.2.22) are summed to produce the output signal

$$
A_{I_{C}}+A_{Q_{B}}=0
$$

The calculations show that the Hartley image rejection architecture can effectively suppress a single sideband without the use of tunable filters. Depending on how the polyphase filter is excited, either the upper or lower sideband is suppressed. For the analysis, a $90^{\circ}$ phase shift is introduced to the in-phase channel relative to the quadrature path. Thus, the lower sideband is suppressed [11].

\subsubsection{Measured image rejection ratio}

The Hartley architecture's IRR is measured using an oscilloscope (Tektronix TDS1002B) and two signal generators which supply the LO and RF signals. The architecture's frequency response is measured for the lower and upper sidebands respectively, where $\omega_{R F}<\omega_{L O}$, and $\omega_{R F}>\omega_{L O}$. The normalised image rejection is presented in Fig. 3.9 and the IRR in Fig. 3.10. Figure 3.10 shows that the IRR in the receiver's passband $(0.977$ $\mathrm{MHz}$ to $1.077 \mathrm{MHz}$ ) is at least $33 \mathrm{~dB}$. 


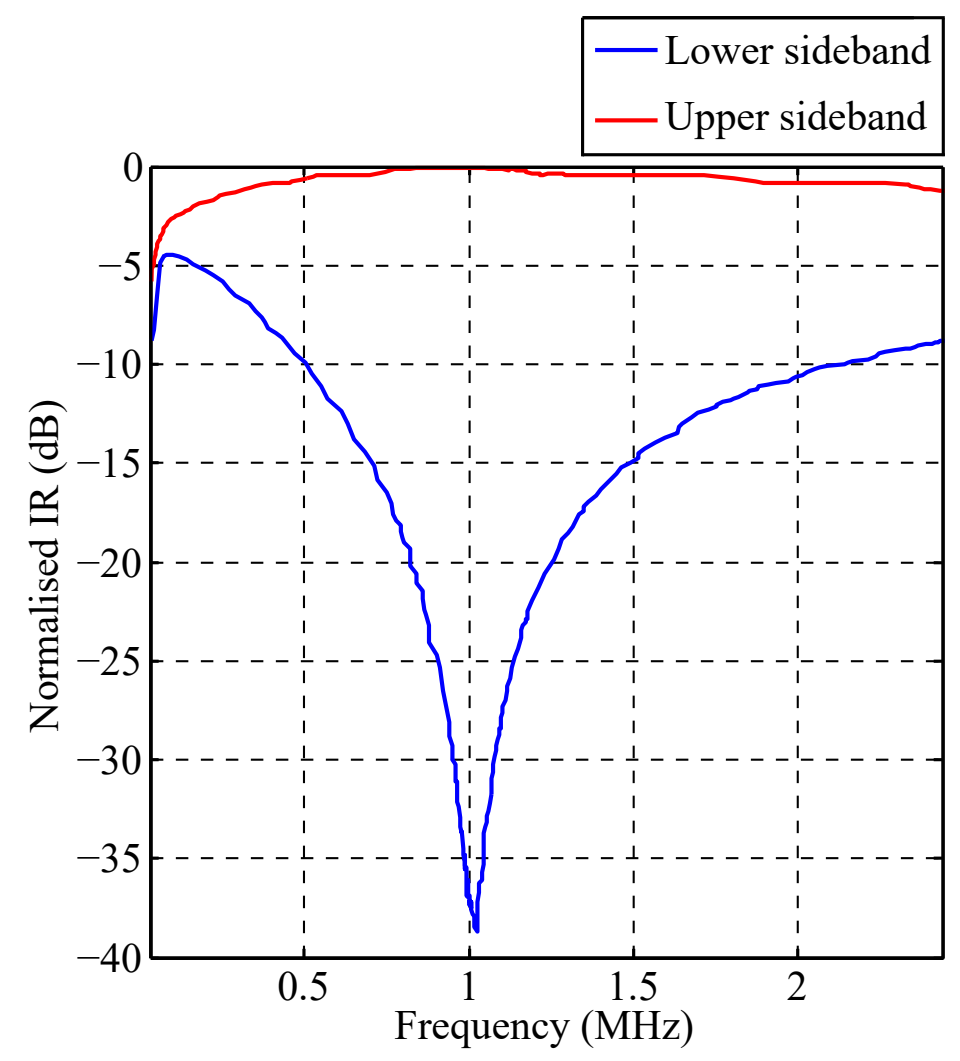

Figure 3.9: Normalised image rejection of the upper and lower sidebands.

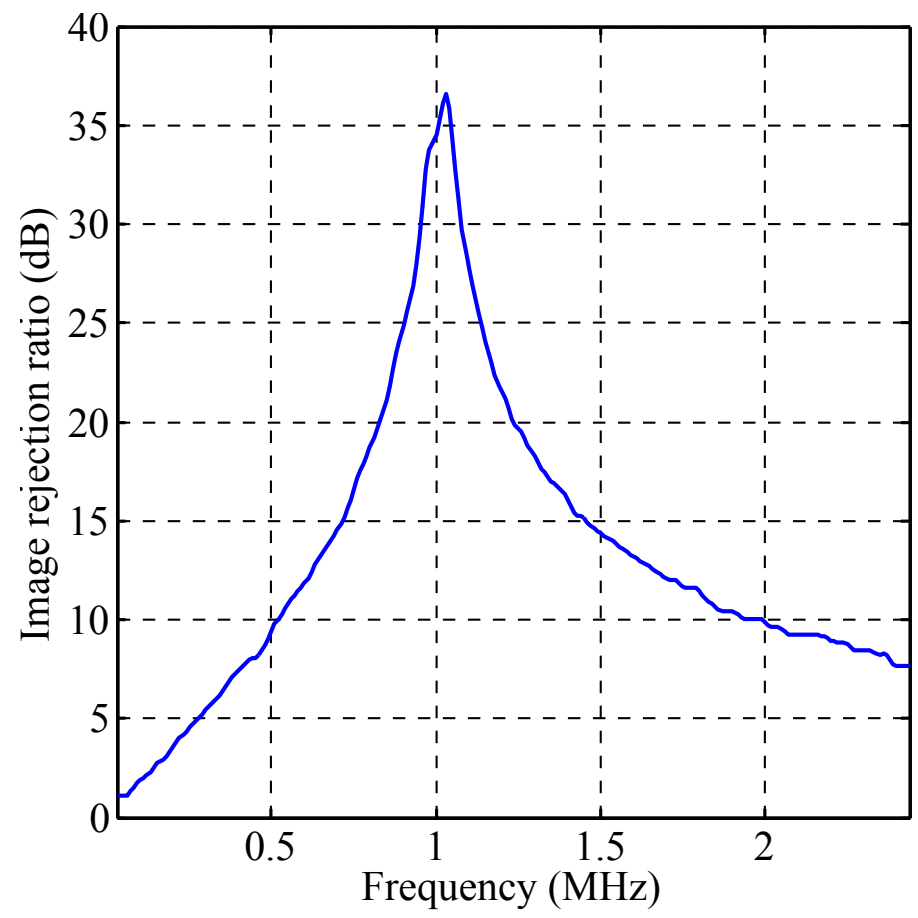

Figure 3.10: The Hartley architecture's measured image rejection ratio.

\subsection{Selecting an analog-to-digital converter}

In general analog-to-digital converters (ADCs) translate analog quantities, which are continuous physical quantities (e.g. voltage levels), to a digital discrete representation of the 
analog value. The ADC's performance has bearing on the following receiver design parameters: the anti-aliasing filter's bandwidth and rejection rate, the receiver gain, and the intermediate frequency $\left(f_{I F}\right)$.

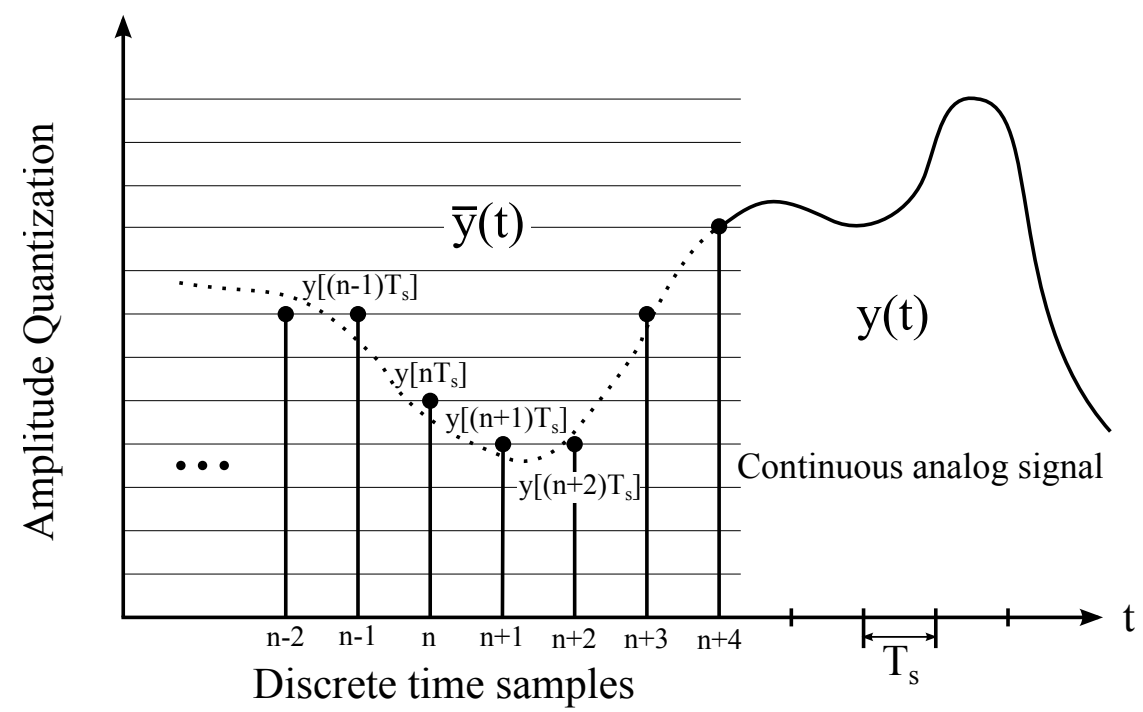

Figure 3.11: Discrete sampling of an continuous analog signal.

The concept of discretizing an analog signal is illustrated in Fig. 3.11. A continuous analog signal is sampled at discrete time intervals, $T_{s}$. The sample frequency $\left(f_{s}=1 / T_{s}\right)$ is carefully chosen to insure an accurate representation of the original analog signal [17].

At higher sample rates the digital representation of the sampled signal becomes more accurate. The minimum sample frequency to unambiguously recover a signal $y(t)$ from its samples $\bar{y}(t)$ is called the Nyquist sample rate [18].

\subsubsection{Differential analog-to-digital converters}

With regards to noise rejection, ADC's with fully differential inputs offer a performance improvement over single-ended inputs. Taking the difference in signal strength between the positive and negative terminals results in common-mode rejection. It is often specified for fully-differential ADCs as a ratio

$$
C M R R=\frac{A_{D I F F}}{A_{C M}}
$$

where CMRR is the common-mode rejection ratio, $A_{D I F F}$ the differential gain, and $A_{C M}$ the common-mode gain. CMRR describes an ADC's ability to reject common-mode signals such as DC offsets or coupled common-mode noise. Examples of common-mode noise sources are noise from $50 / 60 \mathrm{~Hz}$ power lines, power supply ripple, and RF or highfrequency switching noise [19].

CMRR is a frequency dependent parameter and as the common-mode signal frequency increases, the phase matching between the positive and negative terminals to the ADC becomes more critical. The desired signal applied to a differential input requires a relative phase shift of $180^{\circ}$ between the ports. This leads to constructive interference for the desired signals $[20]$. 


\subsubsection{Nyquist-Shannon sampling theorem}

The Nyquist-Shannon sampling theorem states that for unambiguous signal recovery an analog signal with a bandwidth of $\Delta f$ must be sampled at twice the signal's bandwidth

$$
f_{s}>2 \Delta f \text {. }
$$

Usually when referring to baseband sampling the signal bandwidth is defined from $-f_{s} / 2$ to $f_{s} / 2$. Sampling signals at frequencies higher than the Nyquist sample rate is referred to as undersampling (or either, bandpass, harmonic, or super-Nyquist sampling). When undersampling the sampled signal's bandwidth is defined as $\Delta f=f_{1}-f_{0}$ where $f_{1}>f_{0}>f_{s} / 2$. The Nyquist criterion is analogous to the Nyquist-Shannon sampling theorem. It states that if the sample frequency is less than twice the signal bandwidth $\left(f_{s}<2 \Delta f\right)$ the phenomena called aliasing will occur. Undersampling applications use aliasing as a way of sampling signals that are of higher frequency than the Nyquist sampling rate [21].
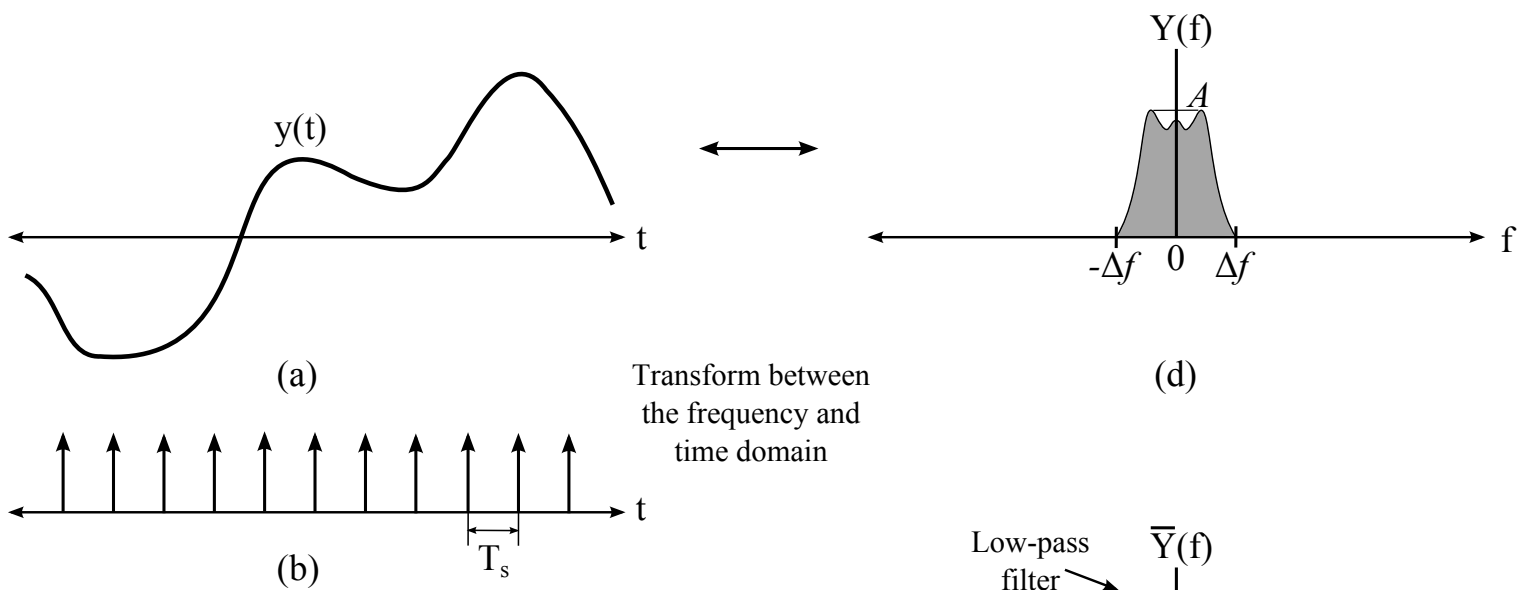

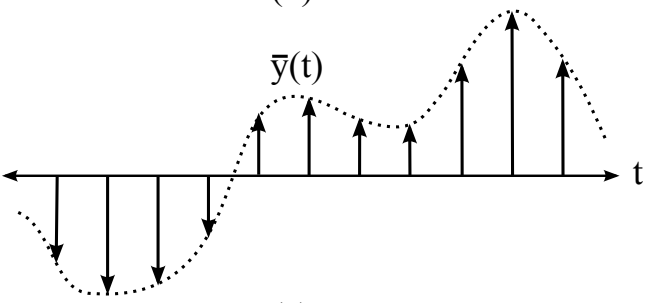

(c)

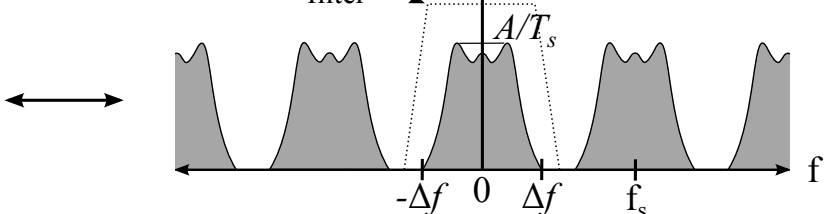

(e)

Figure 3.12: Spectral representation of a quantized continuous baseband signal.

When discretizing a continuous baseband analog signal $y(t)$, as in Fig. 3.11, it is assumed that the signal is band limited to $\Delta f \mathrm{~Hz}$. This is described by Eq. (3.3.3) where $Y(f)$ is the frequency domain representation of the analog signal.

$$
Y(f)=0 \quad \text { for }|f|>\Delta f .
$$

The relationship between the sampled signal $\bar{y}(t)$ and the original analog signal $y(t)$ is given by

$$
\bar{y}(t)=\sum_{n} y\left(n T_{s}\right) \delta\left(t-n T_{s}\right),
$$

where $\delta_{T_{s}}(t)=\sum_{n} \delta\left(t-n T_{s}\right)$ is an unity impulse train (Dirac comb). The impulse train is periodic and repeats every $T_{s}$ seconds, $T_{s}$ is defined as $T_{s}=1 / f_{s}$, and $f_{s}$ is the ADC's sample frequency. Due to the Dirac comb's periodicity, it can be expressed as an exponential 
Fourier series as in Eq. (3.3.5), where $\omega_{s}=2 \pi f_{s}$.

$$
\delta_{T_{s}}(t)=\frac{1}{T_{s}} \sum_{n=-\infty}^{\infty} e^{j n \omega_{s} t}
$$

By applying Eq. (3.3.5) the discretized signal is expressed as

$$
\bar{y}(t)=y(t) \delta_{T_{s}}(t)=\frac{1}{T_{s}} \sum_{n=-\infty}^{\infty} y(t) e^{j n \omega_{s} t} .
$$

The frequency domain representation of the sampled signal provides insight into why aliasing occurs

$$
\bar{Y}(f)=\frac{1}{T_{s}} \sum_{n=-\infty}^{\infty} Y\left(f-n f_{s}\right) .
$$

Equation (3.3.7) is the Fourier transform of $\bar{y}(t)$ producing $\bar{Y}(f)$. The spectrum of $\bar{Y}(f)$ consists of $Y(f)$ scaled by a constant $1 / T_{s}$ and repeated periodically every $f_{s} \mathrm{~Hz}$.

A visual representation of Eqs. (3.3.4) and (3.3.7) is presented in Fig. 3.12. From inspection of Fig. 3.12 (e) it is clear that when the sample frequency is $f_{s}<2 \Delta f$ the spectrum of adjacent replicas will overlap causing aliasing and signal distortion [18].

Aliasing is prevented by applying an anti-aliasing filter which band limits the received signal so that the spectrum conforms to the Nyquist criterion. The anti-aliasing filter requirements and design is discussed in section 3.4.

\subsubsection{Undersampling}

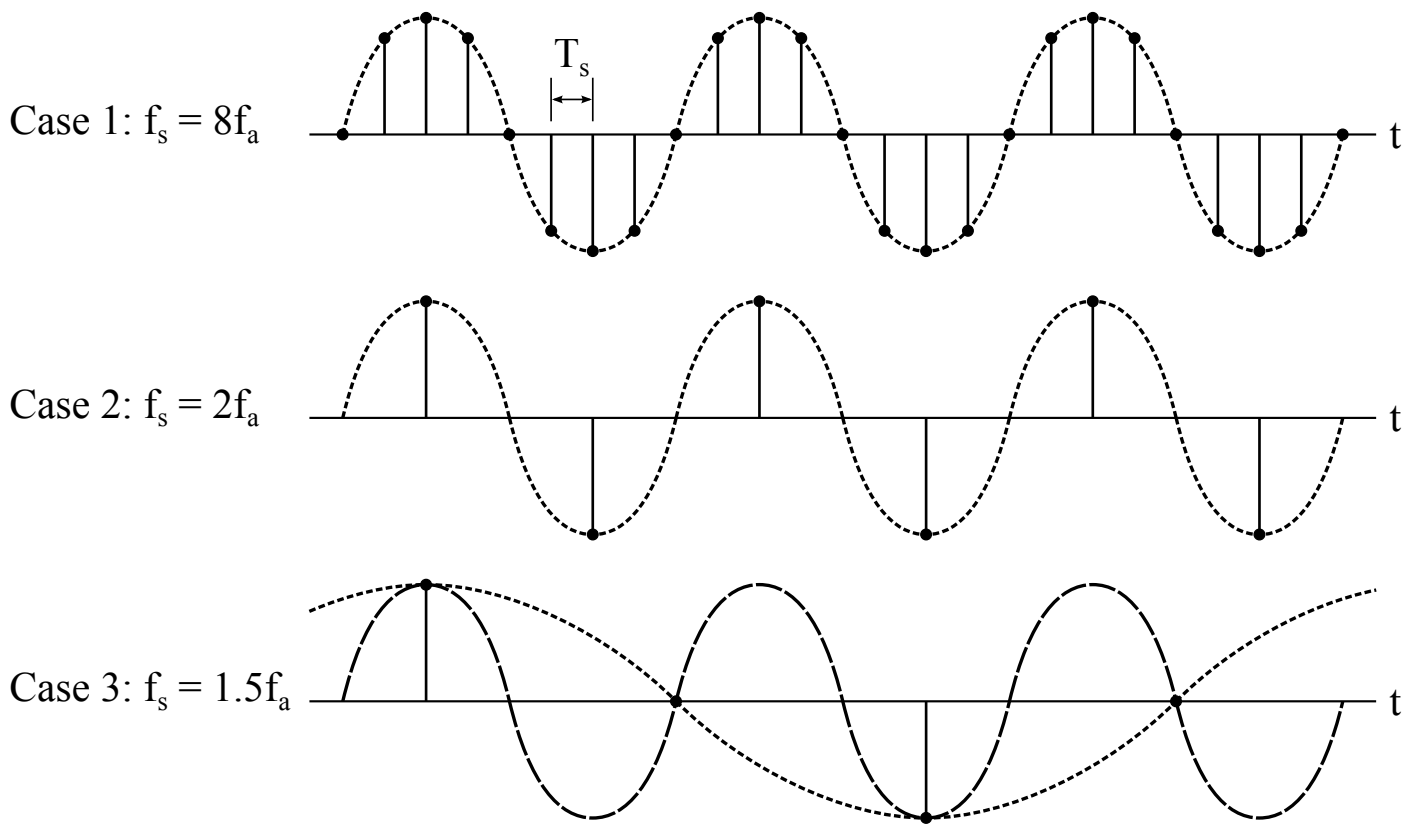

Figure 3.13: Time domain representation of sampling and aliasing.

The process of sampling signals outside the first Nyquist zone is referred to as undersampling. ADCs that sample signals above the first Nyquist zone is widely used in modern communication systems because the process is analogous to analog down-conversion. 


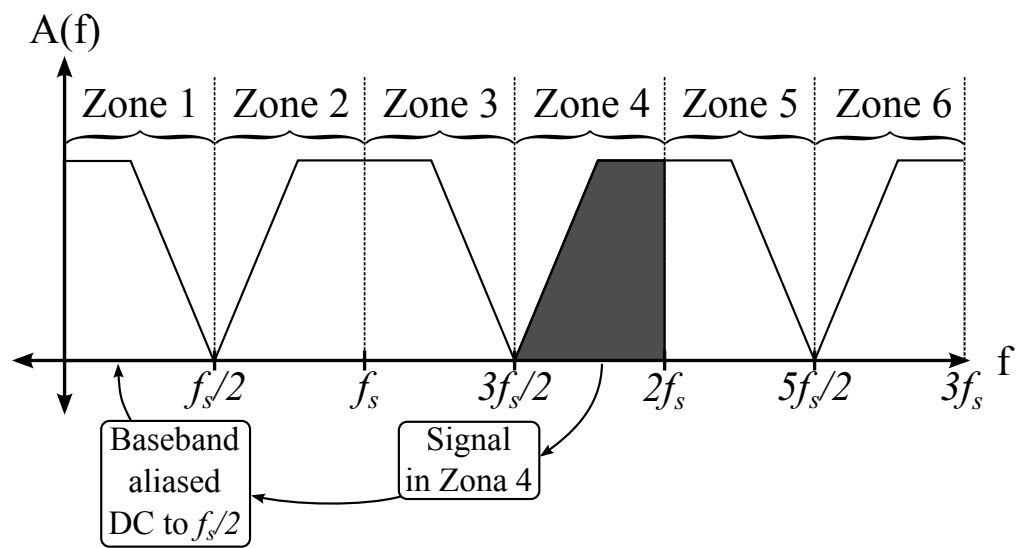

Figure 3.14: Signal aliased from a higher Nyquist zone to baseband.

Super-Nyquist sampling allows direct quantization of IF signals without additional hardware required for demodulation [22].

Figure 3.13 illustrates a signal sampled at different frequencies. For Case 1, the signal is sampled at more than the Nyquist sample frequency. Therefore, the signal information is preserved. Case 2 represents the ambiguous condition where $f_{s}=2 f_{a}$, i.e. $f_{s}=2 \Delta f$. If the relationship between the sample points and the sine wave is such that the samples are precisely at the zero crossings (rather than at the peaks, as shown in Fig. 3.13) all information regarding the sine wave is lost. With regards to case 3 , where $f_{s}<2 f_{a}$ the samples relate to a sine wave with a frequency lower than $f_{s} / 2$, i.e. the signal is aliased into the Nyquist zone between DC and $f_{s} / 2$. As the sample frequency decreases, the aliased signal approaches DC in the frequency spectrum. At DC the aliased signal wraps and starts to increases until $f_{s} / 2$. This back and forth continues as the sample frequency decreases. Therefore, knowing in which Nyquist zone the undersampled signal exists allows calculation of the signal's actual frequency.

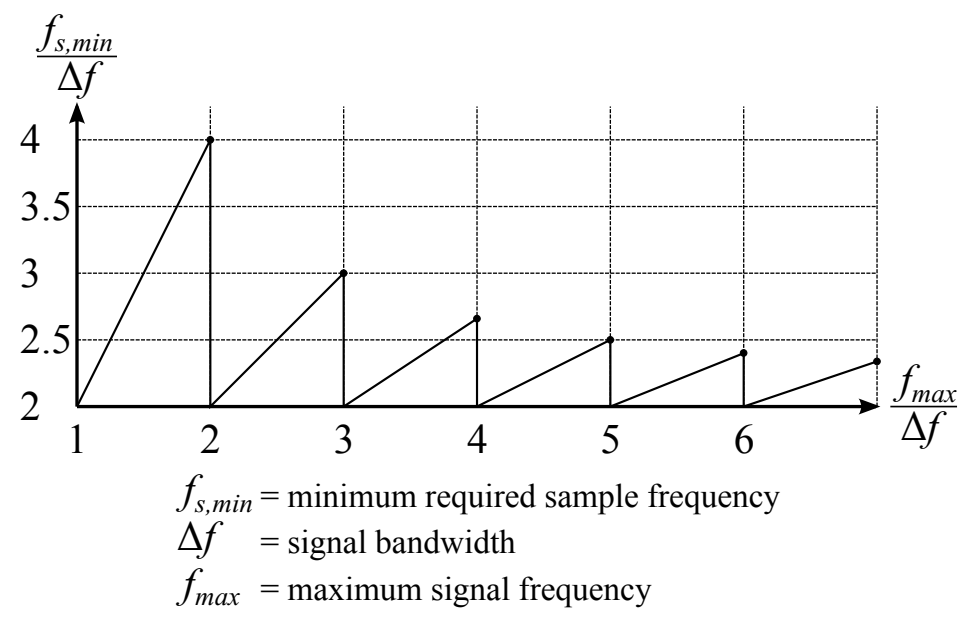

Figure 3.15: Minimum required sample frequency versus the desired signal's maximum frequency scaled to the signal bandwidth.

As depicted in Fig. 3.14 signals in even Nyquist zones are inverted when aliased to baseband. It is possible to correct the inversion in software. A minimum required sample rate is determined to ensure that the bandwidth of interest does not exist in more than one Nyquist zone. The greatest increase in sample frequency is required when the signal bandwidth is at the threshold of wholly crossing from one Nyquist zone to the next as 
calculated by Eq. (3.3.8), where $N_{\text {zone }}$ is the operating Nyquist zone. Figure 3.15 plots the ratio between the minimum required sample rate and the received signals bandwidth as a function of the signal's position in the spectrum.

$$
f_{s, \min }=\left(2+\frac{2}{N_{\text {zone }}}\right) \Delta f
$$

An ADC's performance usually deteriorates as the operating frequency increases beyond the ADC's baseband bandwidth. Therefore, ADC parameters such as input bandwidth, distortion performance and dynamic range is considered in undersampling applications. An ADC suited to undersampling maintains its dynamic range into the higher order Nyquist zones [23].

\subsection{Anti-aliasing filters in undersampling applications}

In this section a method is presented to determine an anti-aliasing filter's minimum requirements in the context of undersampling applications. Two bandpass filters in the receiver chain are designed using these concepts. With reference to Fig. 2.12, the first filter is the SFC and the second an anti-aliasing filter.

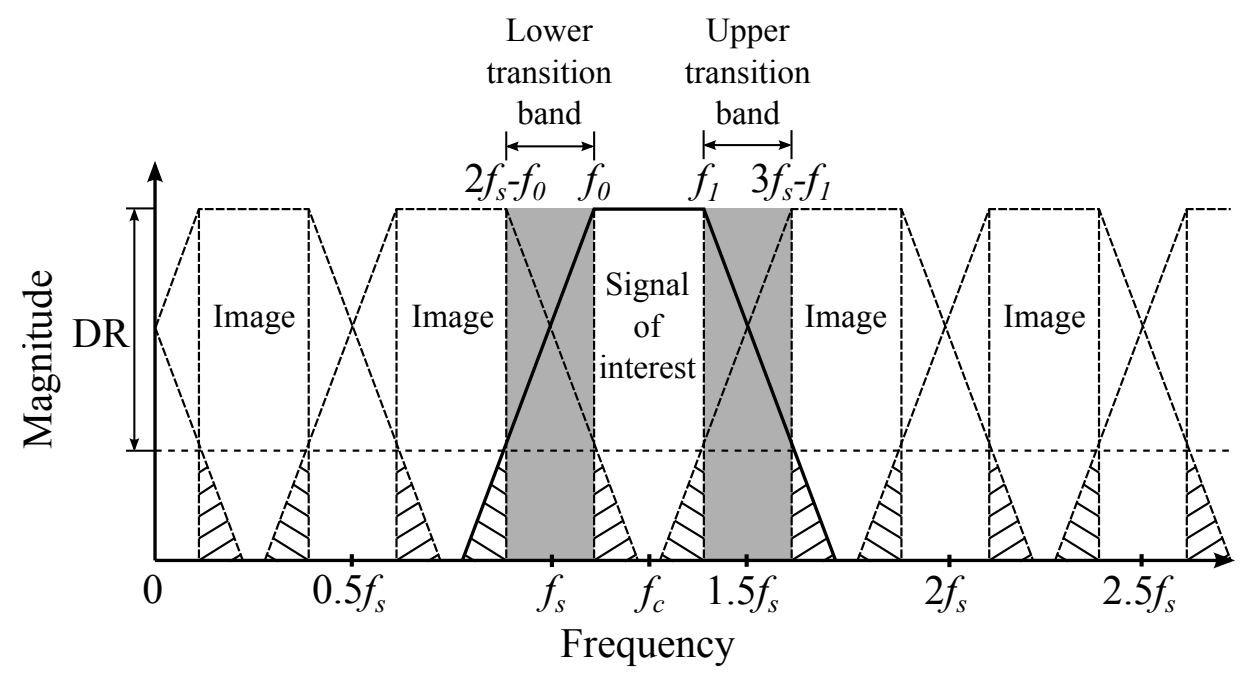

Figure 3.16: Anti-aliasing filter for undersampling.

The ADC's sample frequency is set-up so that the signal bandwidth of interest is in the centre of the third Nyquist zone, as illustrated by Fig. 3.16. The ADC's dynamic range $(\mathrm{DR})$ is $\pm 60 \mathrm{~dB}$ and it dictates the filter's required stopband attenuation. The transition bands, illustrated in Fig. 3.16, are the sections of spectrum in between the signal bandwidth and its neighbouring images. The transition band and desired stopband attenuation describes the anti-aliasing filter's roll-off. The lower transition band stretches from $2 f_{s}-f_{0}$ to $f_{0}$, and the upper band from $f_{1}$ to $3 f_{s}-f_{1}$. The anti-aliasing filter requirements can be relaxed by increasing the sample frequency. However, the carrier frequency $\left(f_{c}\right)$ should be increased proportionally to keep the bandwidth centred in the third Nyquist zone.

Two equations are used to determine the sample frequency. The first equation is the Nyquist criteria as described by Eq. (3.3.2) and the second is Eq. (3.4.1). Equation 
(3.4.1) ensures that the carrier frequency is placed in the centre of a Nyquist zone, where $N_{\text {zone }}=1,2,3, \cdots$ corresponds to the Nyquist zone in which the signal bandwidth is located [23].

$$
f_{s}=\frac{4 f_{c}}{2 N_{\text {zone }}-1}
$$

Using Eq. (3.4.1) the receiver's sample frequency is determined $f_{s}=0.821 \mathrm{MHz}$, where the carrier frequency is $f_{c}=\frac{125 \mathrm{MHz}}{128}+\frac{100 \mathrm{kHz}}{2}=1.027 \mathrm{MHz}$ and the operating Nyquist zone is $N_{\text {zone }}=3$.

Selecting $N_{\text {zone }}$ as large as possible while still maintaining $f_{s}>2 \Delta f$ results in the minimum required sampling rate

$$
\begin{aligned}
2 \Delta f & =\frac{4 f_{c}}{2 N_{\text {zone } \max }-1}, \\
N_{z o n e, \max } & =\frac{f_{c}}{\Delta f}+\frac{1}{2},
\end{aligned}
$$

where $N_{\text {zone,max }}$ is the maximum theoretical operating Nyquist zone. Therefore, an ADC's super-Nyquist sampling performance must be considered when determining the maximum operating Nyquist zone.

\subsection{Intermediate frequency filter}

The IF filter design is presented following a discussion on passive lumped elements. The filter's specifications are determined in this section. It is desired that the IF filter serve a duel purpose as anti-aliasing filter and to provide sensitivity frequency control (SFC).

\subsubsection{Lumped element considerations}

The filter's operating frequency is low enough that passive lumped elements can be used. Therefore the limitations and considerations associated with lumped elements are briefly discussed.

For passive filter designs the network's energy efficiency is an important parameter. Each lumped element's energy efficiency is described by its quality (Q) factor. The Q factor represents the ratio of energy stored to energy dissipated through the element's equivalent series resistance (ESR) [24]. The Q factor of a capacitor is given by

$$
Q_{C}=\frac{X_{C}}{R_{C}}=\frac{1}{\omega C R_{C}},
$$

where $X_{C}$ is the reactance of the capacitor, $R_{C}$ is the ESR, $C$ is the capacitance, and $\omega$ is the frequency of operation in radians. An inductor's $Q$ factor is defined in the same way

$$
Q_{L}=\frac{X_{L}}{R_{L}}=\frac{\omega L}{R_{L}}
$$

where $X_{L}$ is the reactance of the inductor, $R_{L}$ is the ESR, and $L$ is the inductance.

The filter resonator elements are usually the most critical components for the network's energy efficiency. It is found that energy is concentrated in the filter's resonators, therefore more power is exerted through losses in the resonators than the coupling elements. The capacitors used in the filter's resonators have relatively high Q factors of \pm 1000 (as seen 
for ceramic capacitors with a COG (NP0) dielectric [25]), where the measured resonator inductor $\mathrm{Q}$ factors are \pm 130 . The order of magnitude difference in $\mathrm{Q}$ factor does not necessary indicate that the resonator inductors are the primary contributors to the overall filter insertion loss. A component's ESR is the absolute indicator of its ability to spend signal energy, usually in the form of heat.

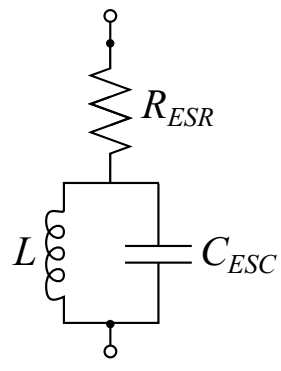

(a)

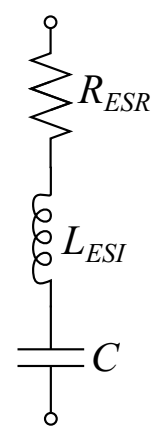

(b)

Figure 3.17: (a) Inductor model, (b) Capacitor model.

The inductor and capacitor models shown in Fig. 3.17 include each component's equivalent parasitic elements. Each lumped element has a self resonant frequency (SRF) at which point the capacitive and inductive impedances cancel each other out leaving only a resistive component [24]. Thus, the SRF of the inductor is given by

$$
S R F_{L}=\frac{1}{2 \pi \sqrt{L C_{E S C}}},
$$

where $C_{E S C}$ is the equivalent shunt capacitance. The capacitor's self resonance is calculated by

$$
S R F_{C}=\frac{1}{2 \pi \sqrt{C L_{E S I}}}
$$

where $L_{E S I}$ is the equivalent series inductance. Beyond the SRF a capacitor starts behaving as an inductor and vice versa.

\subsubsection{Sensitivity frequency control}

The SFC's frequency response is designed to attenuate signals from close proximity targets more than echoes received from targets that are further away. This has a normalising effect on the FMCW radar's received spectrum. The amount of attenuation required is dependent on the transmission power and the object's radar cross section (RCS).

The received signal power is determined using the radar equation

$$
\begin{aligned}
P_{r} & =\frac{P_{t} G^{2} \lambda^{2} \sigma}{(4 \pi)^{3} R^{4}}, \\
10 \log \left(P_{r}\right) & =10 \log \left[\frac{P_{t} G^{2} \lambda^{2} \sigma}{(4 \pi)^{3}}\right]-40 \log (R),
\end{aligned}
$$

where $P_{t}$ is transmission power (watts), $P_{r}$ is received power (watts), $G$ is the antenna gain, $\lambda$ is the signal wave length, $\sigma$ is a target's RCS $\left(m^{2}\right)$, and $R$ is the distance to the target [1]. 
As discussed in Chapter 1, an FMCW radar's range is related to beat frequency by the following equation

$$
R=\frac{f_{b} v_{p} T_{m}}{2 \Delta f}
$$

By substituting Eq. (3.5.6) into Eq. (3.5.5) it is shown that propagating electromagnetic waves attenuate at $40 \mathrm{~dB}$ per decade. It is assumed that the variables in term one of Eq. (3.5.7) is frequency independent. At baseband, Eq. (3.5.7) is normalised (with regards to frequency) by adding a filter with an amplitude response that increases by $40 \mathrm{~dB}$ per decade. Normalisation of the spectrum is usually the intended function of an SFC.

$$
10 \log \left(P_{r}\right)=10 \log \left[\frac{P_{t} G^{2} \lambda^{2} \sigma}{(4 \pi)^{3}\left(\frac{v_{p} T_{m}}{2 \Delta f}\right)^{4}}\right]-40 \log \left(f_{b}\right)
$$

\subsubsection{Intermediate frequency filter specifications}

As stated in section 3.4 the desired signal has a $100 \mathrm{kHz}$ bandwidth $(\Delta f=100 \mathrm{kHz})$ and is centred around $f_{c}$, where $f_{c}=1.027 \mathrm{MHz}$. The ADC is set-up to undersample the desired spectrum at a frequency of $f_{s}=821 \mathrm{kHz}$ as calculated using Eq. (3.4.1).

It is shown in section 5.2 that the cascaded RF components have a greater dynamic range than the $\mathrm{ADC}$. Consequently, the receiver's dynamic range is restricted to $60 \mathrm{~dB}$ by the ADC. To prevent aliasing, the IF filter is designed to have more than $60 \mathrm{~dB}$ attenuation in the neighbouring Nyquist zones.

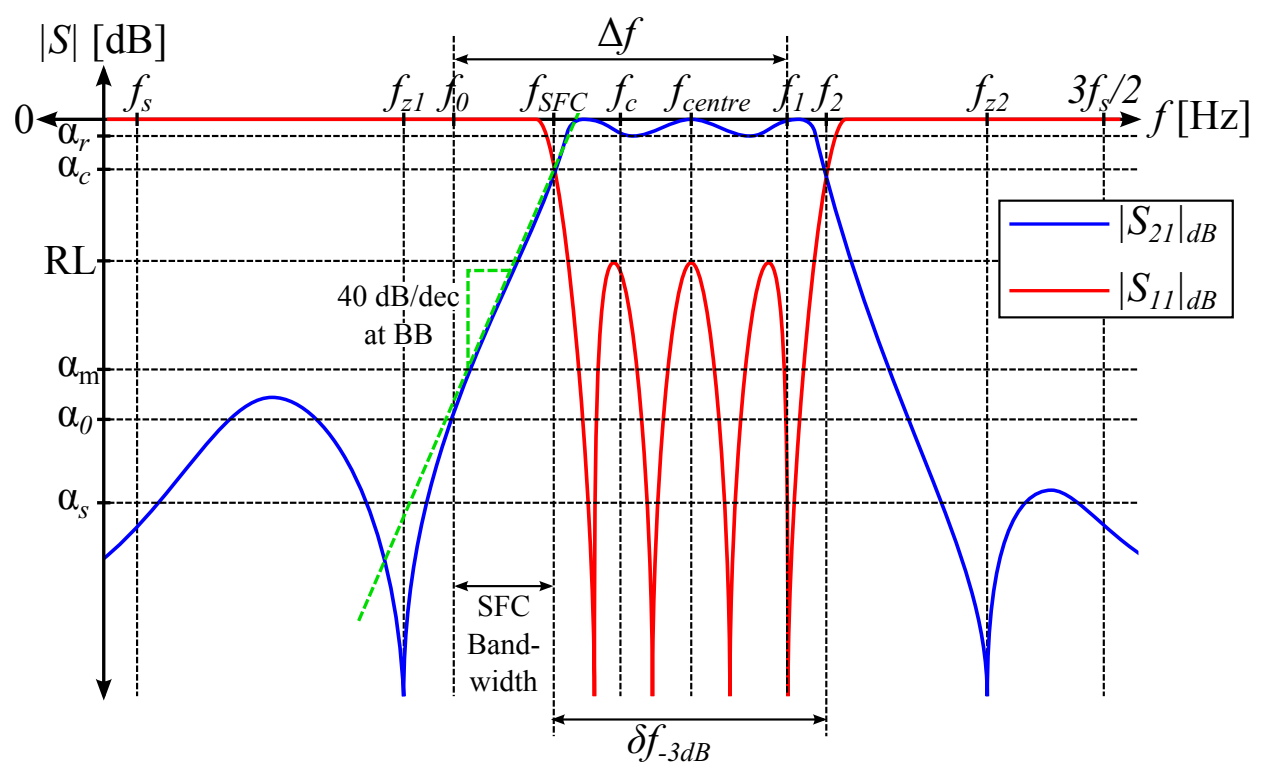

Figure 3.18: Representation of the desired intermediate frequency filter characteristics.

The SFC attenuation degrades the receiver chain's noise figure as shown in Chapter 4. Therefore, the IF filter is designed to partially suppress and normalise the desired spectrum. The signal bandwidth of interest is defined from frequency $f_{0}$ to $f_{1}$ as illustrated in Fig. 3.18, i.e. $\Delta f=f_{1}-f_{0}$. The strongest reflections with the potential to saturate the receiver are found at low beat frequencies, $f_{0}$ to $f_{S F C}$. These reflections are suppressed 
by the SFC. However, maximum sensitivity is desired in the section of spectrum that is expected to contain weak echoes $\left(f_{S F C}\right.$ to $\left.f_{1}\right)$.

Frequency $f_{0}$ in Fig. 3.18 is equal to the IF,

$$
f_{0}=f_{I F} .
$$

Thus, $f_{0}$ relates to the IF FMCW radar's zero beat frequency. For the design, the SFC is set-up to operate in half of the desired bandwidth,

$$
f_{S F C}=f_{0}+0.5 \Delta f=1.0266 \mathrm{MHz} .
$$

The range to a target with a beat frequency of

$$
f_{b, S F C}=0.5 \Delta f=50 \mathrm{kHz}
$$

is calculated using Eq. (3.5.6). Assuming propagation in free space the target is at a distance of $18.75 \mathrm{~m}$.

As discussed in section 3.5.2, the SFC baseband roll-off should be $40 \mathrm{~dB} /$ decade to normalise the received power in a section of spectrum. The $40 \mathrm{~dB} /$ decade baseband rolloff is translated to the IF by ensuring the magnitude of the beat frequency, $f_{0}+\frac{f_{b, S F C}}{10}$, is $40 \mathrm{~dB}$ lower than the magnitude at $f_{0}+f_{b, S F C}$. The desired baseband behaviour is approximated at the IF by adding two finite frequency transmission zeros to the filter response.

A MATLAB script generates the polynomials needed to realise a Chebyshev filter response that has the specified characteristics. It is found that placing a transmission zero at the IF (i.e. zero beat frequency $f_{0}$ ) produces the desired roll-off,

$$
f_{z 1}=f_{0} .
$$

The synthesised filter's return loss (RL) is $16 \mathrm{~dB}$. By tuning the RL the passband ripple and out-of-band attenuation is influenced. The filter's equiripple magnitude must be as low as possible and the out-of-band attenuation no less than the SFC's attenuation. The passband ripple $\left(A_{r}\right)$ is related to RL by Eq. (3.5.12) and a maximum ripple of 0.11 $\mathrm{dB}$ is calculated [26].

$$
\begin{aligned}
10^{A_{r} / 10}-1 & =\frac{1}{10^{R L / 10}-1} \\
A_{r} & =10 \log _{10}\left(\frac{1}{10^{R L / 10}-1}+1\right) \\
& =-\alpha_{r}
\end{aligned}
$$

During the filter synthesis the low pass prototype's zero frequency maps to the bandpass filter's centre frequency at

$$
f_{\text {centre }}=\frac{f_{S F C}+f_{2}}{2}=1.0616 \mathrm{MHz} .
$$

The parameters in Table 3.1 are used during the IF filter synthesis and describe a symmetrical amplitude response. The filter's cut-off frequency at $f_{2}$ is slightly higher than the bandwidth requirement. This ensures minimum attenuation at $f_{1}$.

Further experimentation with regards to the IF filter's response is regarded as future work. It might be beneficial to normalise the entire desired spectrum by expanding the SFC bandwidth. 
Table 3.1: Intermediate frequency filter parameters (refer to Fig. 3.18)

\begin{tabular}{c|c|c}
\hline \hline $\begin{array}{c}\text { Filter } \\
\text { parameter }\end{array}$ & $\begin{array}{c}\text { Description } \\
\text { of magnitude }\end{array}$ & $\begin{array}{c}\text { Value } \\
{[\mathrm{dB}]}\end{array}$ \\
\hline$\alpha_{r}$ & Ripple & -0.11 \\
$\alpha_{c}$ & Cut-off & -3 \\
$\mathrm{RL}$ & Passband return loss & -16 \\
$\alpha_{0}$ & SFC att. & -40 \\
$\alpha_{m}$ & Minimum out-of-band att. & $\alpha_{0}$ \\
$\alpha_{s}$ & Anti-aliasing & -60 \\
\hline \hline Filter & Description & Value \\
parameter & of frequency & {$[\mathrm{MHz}]$} \\
\hline$f_{s}$ & ADC sample freq. & 0.821 \\
$f_{I F}$ & Intermediate frequency & 0.9766 \\
$f_{0}$ & Start of band & $f_{I F}$ \\
$f_{z 1}$ & Transmission zero 1 & $f_{0}$ \\
$f_{S F C}$ & End of SFC band & 1.0266 \\
$f_{c}$ & Centre of band & 1.027 \\
$f_{c e n t r e}$ & Centre of band-pass filter & 1.0616 \\
$f_{1}$ & End of receiver band & 1.0766 \\
$f_{2}$ & Filter cut-off frequency & 1.0966 \\
$f_{z 2}$ & Transmission zero 2 & 1.1466 \\
$\Delta f$ & Receiver bandwidth & 0.1 \\
$\delta f_{-3 d B}$ & Filter bandwidth & 0.070 \\
\hline & &
\end{tabular}

\subsubsection{Intermediate frequency filter synthesis}

The IF filter synthesis is essentially a three step process. First, the polynomials required to realise the desired Chebyshev filter response is generated. Then a coupling matrix is synthesised for a realisable double terminated network. Finally, the passive lumped element values for a narrow band coupled resonator filter is produced from the coupling matrix [27].

\section{Synthesis of the Chebyshev filter function}

For a two-port network, the voltage scattering matrix $[S]$ is represented by a $2 \times 2$ matrix

$$
\left[\begin{array}{c}
V_{1}^{-} \\
V_{2}^{-}
\end{array}\right]=\left[\begin{array}{ll}
S_{11} & S_{12} \\
S_{21} & S_{22}
\end{array}\right]\left[\begin{array}{c}
V_{1}^{+} \\
V_{2}^{+}
\end{array}\right]
$$

or

$$
\left[V^{-}\right]=[S]\left[V^{+}\right]
$$

where $V_{n}^{+}$is the amplitude of the voltage wave incident on port $n$ and $V_{n}^{-}$is the amplitude of the voltage wave reflected from port $n$ [8].

Assuming the network is passive, lossless, and reciprocal, the scattering matrix yields the following equations that describe the conservation of energy. The sum of the reflection and transmission parameters at each port is unity and produces

$$
\begin{aligned}
& S_{11}(s) S_{11}(s)^{*}+S_{21}(s) S_{21}(s)^{*}=1, \\
& S_{22}(s) S_{22}(s)^{*}+S_{12}(s) S_{12}(s)^{*}=1,
\end{aligned}
$$


and an orthogonality equation

$$
S_{11}(s) S_{12}(s)^{*}+S_{21}(s) S_{22}(s)^{*}=0
$$

where the S-parameters are assumed to be functions of the complex frequency variable, $s=j \omega[28]$.

A lossless two-port network's reflection and transmission coefficients can be expressed as the ratio of two polynomials [28]. Therefore, the reflection parameter $S_{11}(s)$ at port 1 of the network is represented by the ratio of two finite-degree polynomials $E(s)$ and $F(s)$,

$$
S_{11}(s)=\frac{F(s)}{\epsilon_{R} E(s)},
$$

where $\epsilon_{R}$ is a real constant that normalises the highest degree coefficients of $E(s)$ and $F(s)$ to unity. By reorganising Eq. (3.5.16) and substituting Eq. (3.5.19), we obtain

$$
\begin{aligned}
S_{21}(s) S_{21}(s)^{*} & =1-\frac{F(s) F(s)^{*}}{\epsilon_{R} E(s) E(s)^{*}}, \\
& =\frac{P(s) P(s)^{*}}{\epsilon^{2} E(s) E(s)^{*}} .
\end{aligned}
$$

The transmission parameter $S_{21}(s)$ is also expressed as the ratio of two polynomials

$$
S_{21}(s)=\frac{P(s)}{\epsilon E(s)},
$$

where

$$
E(s) E(s)^{*}=\frac{P(s) P(s)^{*}}{\epsilon^{2}}+\frac{F(s) F(s)^{*}}{\epsilon_{R}^{2}} .
$$

From Eqs. (3.5.19) and (3.5.21) it is seen that the S-parameters share a common denominator polynomial $E(s)$, where $E(s), P(s)$ and $F(s)$ are $N$ th-degree polynomials with complex coefficients (for lowpass and bandpass filters). Parameter $N$ is the order of the filter network under consideration [28].

Under the assumption that a lossless passive network is described by these polynomials, $E(s)$ should be strictly Hurwitz, meaning that all the roots of $E(s)$ [poles of $S_{11}(s)$ and $S_{21}(s)$ ] should be the left half of the complex plane. However, these poles need not be symmetrical about the real axis in the complex plain [28].

The roots of the numerator polynomial $F(s)$ of $S_{11}(s)$ are the points of zero reflected power, or perfect transmission. The transmission zeros of the filter function are the zeros described by the numerator polynomial $P(s)$ of $S_{21}(s)$. The degree $n_{z f}$ of the polynomial $P(s)$ matches the number of finite transmission zeros that the filter transfer function incorporates. This implies that $n_{z f} \leq N$, because $S_{21}(s)$ may not exceed unity for a passive network as $s \rightarrow j \infty$. Note, because of the orthogonality condition both polynomials $[F(s)$ and $P(s)$ ] must have zeros that are either on the imaginary axis of the complex S-plane or in mirror image pairs symmetrically arranged about the imaginary axis. However, to determine the denominator polynomial $E(s)$ the alternating pole method is applied as described by [28], therefore reducing Eq. (3.5.22) to

$$
\epsilon^{2} \epsilon_{R}^{2} E(s) E(s)^{*}=\left[\epsilon_{R} P(s)+\epsilon F(s)\right]\left[\epsilon_{R} P(s)+\epsilon F(s)\right]^{*},
$$

for odd values of $N-n_{f z}$, and

$$
\epsilon^{2} \epsilon_{R}^{2} E(s) E(s)^{*}=\left[\epsilon_{R} j P(s)+\epsilon F(s)\right]\left[\epsilon_{R} j P(s)+\epsilon F(s)\right]^{*},
$$


when $N-n_{f z}$ is even. Equations (3.5.23) and (3.5.24) show that by selecting the position of the $N$ singularities in the left half of the S-plane, the denominator polynomial $E(s)$ can be formed. The alternating pole method is valid on the condition that the reflection zeros of $F(s)$ lie on the imaginary axis.

By definition a Chebyshev function's reflection zeros [roots of $F(s)$ ] are on the imaginary axis of the complex S-plane (or real axis of the $\omega$ plane). Therefore, the alternating pole formula is valid for a lossless network described by the Chebyshev function as follows

$$
\begin{aligned}
S_{21}(\omega) S_{21}(\omega)^{*} & =\frac{P(\omega) P(\omega)^{*}}{\epsilon^{2} E(\omega) E(\omega)^{*}}, \\
& =\frac{1}{\left[1-j \frac{\epsilon}{\epsilon_{R}} k C_{N}(\omega)\right]\left[1+j \frac{\epsilon}{\epsilon_{R}} k C_{N}(\omega)^{*}\right]},
\end{aligned}
$$

where

$$
k C_{N}(\omega)=\frac{F(\omega)}{P(\omega)}
$$

and where $k$ is a normalising constant, and $\omega$ is the real frequency that is related to the complex frequency variable $s=j \omega$. The real constant $\epsilon$ is determined by evaluating $P(s) / E(s)$ at a convenient value of $\omega$. For the Chebyshev filter function, $\epsilon$ is a constant that normalises $S_{21}(\omega)$ to the desired equiripple level $\left(A_{r}\right)$ at $\omega= \pm 1$ [28],

$$
\begin{aligned}
\left|S_{21}(\omega)\right|_{\omega= \pm 1}^{2} & =\left|\frac{P(\omega) / \epsilon}{E(\omega)}\right|_{\omega= \pm 1}^{2}, \\
\frac{|P(\omega)|_{\omega= \pm 1}^{2}}{|P(\omega)|_{\omega= \pm 1}^{2}+\frac{\epsilon^{2}}{\epsilon_{R}^{2}}|F(\omega)|_{\omega= \pm 1}^{2}} & =10^{-A_{r 1} / 10},
\end{aligned}
$$

and solving for $\epsilon$,

$$
\epsilon=\sqrt{10^{A_{r 1} / 10}-1}\left|\frac{P(\omega)}{F(\omega) / \epsilon_{R}}\right|_{\omega= \pm 1},
$$

where $A_{r 1}$ is the prescribed equiripple level in $\mathrm{dB}$ at $\omega= \pm 1$. Equation (3.5.28) is expressed in terms of return loss by applying Eq. (3.5.12) resulting in

$$
\epsilon=\frac{1}{\sqrt{10^{R L / 10}-1}}\left|\frac{P(\omega)}{F(\omega) / \epsilon_{R}}\right|_{\omega= \pm 1} .
$$

The maximum level of $\left|S_{21}(s)\right|$ for a passive network is 1 (or $0 \mathrm{~dB}$ ). If $n_{f z}<N$ [i.e., $E(s)$ is a higher order polynomial than $P(s)]$ it follows that $\left|S_{21}(s)\right|=0$ at $s= \pm j \infty$. When $\left|S_{21}(j \infty)\right|=0$ the conservation of energy dictates that

$$
S_{11}(j \infty)=\frac{1}{\epsilon_{R}}\left|\frac{F(j \infty)}{E(j \infty)}\right|=1 .
$$

With reference to Eq. (3.5.30), if $n_{f z}<N$ the highest degree coefficients of $E(s)$ and $F(s)$ are normalised to 1 , thus $\epsilon_{R}=1$.

When $P(s)$ is a $N$ th-degree polynomial (fully canonical function), where all $N$ available transmission zeros are at finite positions in the complex plane $\left(n_{f z}=N\right)$, the attenuation at infinite frequency $(s= \pm j \infty)$ is finite. For the fully canonical case, $E(s), F(s)$, and 
$P(s)$ are all $N$ th-degree polynomials and their highest degree coefficients are unity. By assessing the conservation of energy condition at $s= \pm j \infty, \epsilon_{R}$ is derived for the fully canonical case,

$$
\begin{gathered}
S_{11}(j \infty) S_{11}(j \infty)^{*}+S_{21}(j \infty) S_{21}(j \infty)^{*}=1, \\
\frac{F(j \infty) F(j \infty)^{*}}{\epsilon_{R}^{2} E(j \infty) E(j \infty)^{*}}+\frac{P(j \infty) P(j \infty)^{*}}{\epsilon^{2} E(j \infty) E(j \infty)^{*}}=1, \\
\frac{1}{\epsilon_{R}^{2}}+\frac{1}{\epsilon^{2}}=1 \text { or } \epsilon_{R}=\frac{\epsilon}{\sqrt{\epsilon^{2}-1}} .
\end{gathered}
$$

$C_{N}(\omega)$ is the filter function of degree $N$, where its poles and zeros are the roots of $P(\omega)$ and $F(\omega)$ respectively. The general Chebyshev characteristic equation has the form

$$
C_{N}(\omega)=\cosh \left[\sum_{n=1}^{N} \cosh ^{-1}\left(x_{n}(\omega)\right)\right] .
$$

To represent a Chebyshev function, $x_{n}(\omega)$ requires the following properties: At $\omega=\omega_{n}$, where $\omega_{n}$ is the position of a transmission zero $x_{n}\left(\omega_{n}\right)= \pm \infty$. For $\omega= \pm 1 x_{n}(\omega)= \pm 1$, and between $\omega=-1$ and $\omega=1$ (in-band), $1 \geq x_{n}(\omega) \geq-1$. Equation (3.5.33) is found to satisfy all the requirements [28].

$$
x_{n}(\omega)=\frac{\omega-1 / \omega_{n}}{1-\omega / \omega_{n}}
$$

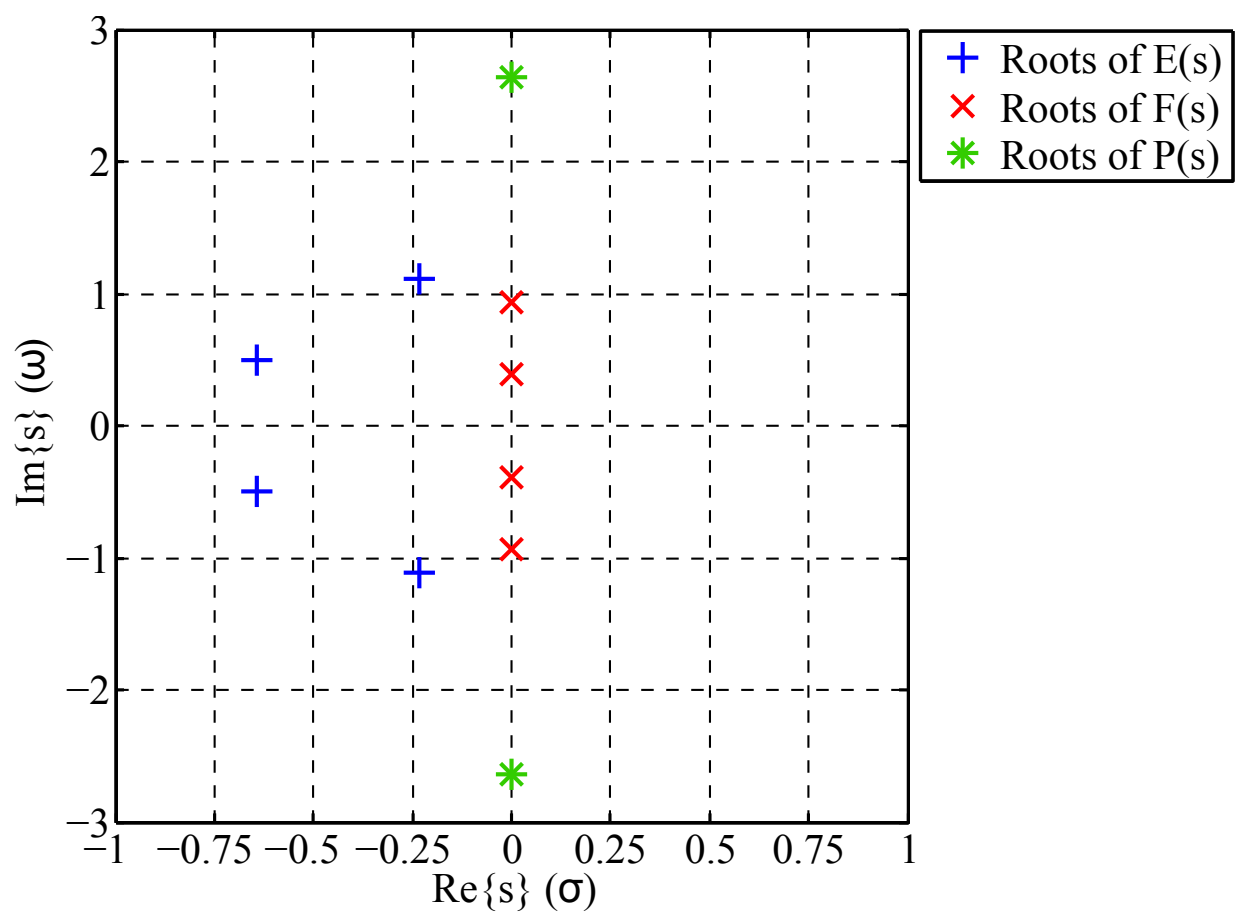

Figure 3.19: The roots of the $E(s), P(s)$ and $F(s)$ polynomials in the complex S-plane.

A recursive technique to calculate the numerator of Eq. (3.5.32) is described in [28] and [29]. Polynomial $F(\omega)$ is determined because it has the same zeros as the numerator of $C_{N}(\omega)$. The filter's prescribed transmission zero's are used as roots to determine polynomial $P(\omega) / \epsilon$. Lastly, the denominator polynomial $E(\omega)$ is constructed by applying the alternating singularity principle [Eqs. (3.5.23) or (3.5.24)]. 


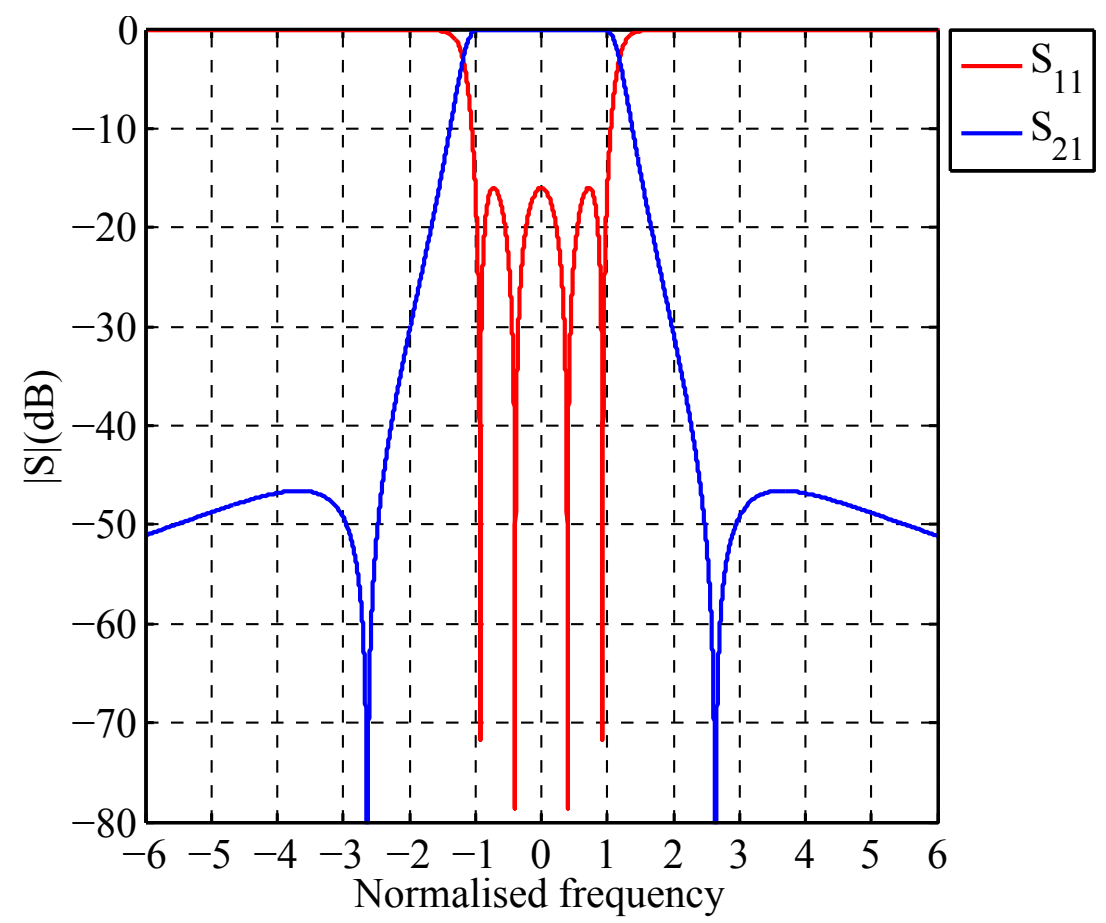

Figure 3.20: Magnitude of $S_{11}$ and $S_{21}$ of the synthesised Chebyshev filter versus frequency.

A MATLAB script provided in Appendix B.1 generates the coefficients of polynomials $E(s), P(s)$, and $F(s)$ that satisfy the IF filter specifications given in section 3.5.3 from the parameters specified in Table 3.1. The corresponding roots of the polynomials are presented in Fig. 3.19 and the IF filter's S-parameters in Fig. 3.20.

\section{Fully-canonical coupling matrix}

A synthesised filter can be represented by a coupling matrix. Inter-resonator couplings directly correspond to the non-zero elements of the matrix. Therefore, by mathematically manipulating the coupling matrix many different filter topologies can be realised [29].

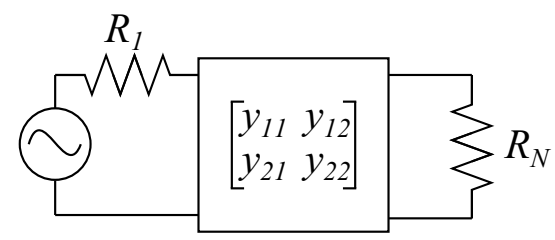

Figure 3.21: General two-port lossless network described by Y-parameters.

In this section the generalised fully-canonical or $N+2$ folded coupling matrix is synthesised. The fully-canonical representation allows for multiple input/output couplings. These couplings are made from the source and/or load to the resonators. The coupling matrix is synthesised by producing two different representations of the filter network's two-port short-circuit admittance matrix $\left[Y_{N}\right]$ (i.e. the Y-parameter matrix). The resultant $\left[Y_{N}\right]$ matrices are then equated [30]. 
Case 1 - Synthesis of the Y-parameter matrix $\left[Y_{N}\right]$ from the transmission and reflection coefficients

The first approach is to derived the $\left[Y_{N}\right]$ matrix from the coefficients of the rational polynomials of the transmission and reflection scattering parameters, $S_{21}(s)$ and $S_{11}(s)$ respectively, where the S-parameters describe the filter's characteristics.

The input impedance to the network shown in Fig. 3.21 is

$$
Z_{\text {in }}(s)=R_{1} \frac{1+S_{11}(s)}{1-S_{11}(s)},
$$

where the matched loads are normalised to $1 \Omega$ with $R_{1}=R_{N}=1 \Omega$. By applying Eq. (3.5.19) the input impedance is rewritten as

$$
\begin{aligned}
Z_{\text {in }}(s) & =\frac{\epsilon_{R} E(s) \pm F(s)}{\epsilon_{R} E(s) \mp F(s)}=\frac{m_{1}+n_{1}}{m_{2}+n_{2}}, \\
& = \begin{cases}\frac{n_{1}}{m_{2}+n_{2}}\left(\frac{m_{1}}{n_{1}}+1\right), & \text { for } N \text { even } \\
\frac{m_{1}}{m_{2}+n_{2}}\left(\frac{n_{1}}{m_{1}}+1\right), & \text { for } N \text { odd }\end{cases}
\end{aligned}
$$

where $m_{1}, m_{2}$ and $n_{1}, n_{2}$ are complex-even and complex-odd polynomials, respectively, constructed from $E(s)$ and $F(s)$ [29]. The input impedance of the lossless network can be represented in terms of $\mathrm{Y}$ and $\mathrm{Z}$-parameters as

$$
Z_{\text {in }}(s)=\frac{Z_{11}}{Z_{22}+1}\left(\frac{1}{y_{22}}+1\right) .
$$

By comparing Eqs. (3.5.35) and (3.5.36) it is seen that

$$
y_{22}(s)=\left\{\begin{array}{ll}
\frac{n_{1}}{m_{1}}=\frac{y_{22 n}}{y_{d}}, & \text { for } N \text { even } \\
\frac{m_{1}}{n_{1}}=\frac{y_{22 n}}{y_{d}}, & \text { for } N \text { odd }
\end{array},\right.
$$

and because the denominator of $y_{21}$ is the same as that of $y_{22}$, and the numerator of $y_{21}$ has the same transmission zeros as $S_{21}(s)$

$$
y_{21}(s)=\left\{\begin{array}{ll}
\frac{P(s) / \epsilon}{m_{1}}=\frac{y_{21 n}}{y_{d}}, & \text { for } N \text { even } \\
\frac{P(s) / \epsilon}{n_{1}}=\frac{y_{21 n}}{y_{d}}, & \text { for } N \text { odd }
\end{array} .\right.
$$

To ensure that both $m_{1}$ and $n_{1}$ have purely real or imaginary coefficients they are constructed from $E(s)$ and $F(s)$ using the numerator of Eq. (3.5.35) as follows:

$$
m_{1}+n_{1}=\epsilon_{R} E(s)+F(s) .
$$

Then,

$$
m_{1}=\operatorname{Re}\left\{e_{0}+f_{0}\right\}+\operatorname{Im}\left\{e_{1}+f_{1}\right\} s+\operatorname{Re}\left\{e_{2}+f_{2}\right\} s^{2}+\cdots,
$$

and

$$
n_{1}=\operatorname{Im}\left\{e_{0}+f_{0}\right\}+\operatorname{Re}\left\{e_{1}+f_{1}\right\} s+\operatorname{Im}\left\{e_{2}+f_{2}\right\} s^{2}+\cdots,
$$

where $e_{i}$ and $f_{i}, i=0,1,2,3 \cdots N$ are the complex coefficients of $E(s)$ and $F(s)$ [29]. 
The procedure followed to generate $y_{22}(s)$ and $y_{21}(s)$ is different depending on whether the filter order $(N)$ is even or odd. The highest degree coefficient in the polynomials $m_{1}$ and $n_{1}$ are unity and by extension those coefficients are real. By following this procedure the denominator polynomials of Eqs. (3.5.37) and (3.5.38) have equal or greater orders when compared to those of the nominators.

By knowing the numerator and denominator polynomials for $y_{21}(s)$ and $y_{22}(s)$, their resultant residues $r_{21 k}$ and $r_{22 k}$ is found by calculating the partial fraction expressions for $y_{21}(s)$ and $y_{22}(s)$. The real eigenvalues $\lambda_{k}$ of the network is determined by rooting the common denominator $y_{d}(s)$ of both $y_{21}(s)$ and $y_{22}(s)$. Expressing the residues in matrix form yields the following equation for the Y-parameter matrix,

$$
\begin{aligned}
{\left[Y_{N}\right] } & =\left[\begin{array}{ll}
y_{11}(s) & y_{12}(s) \\
y_{21}(s) & y_{22}(s)
\end{array}\right], \\
& =\frac{1}{y_{d}(s)}\left[\begin{array}{ll}
y_{11 n}(s) & y_{12 n}(s) \\
y_{21 n}(s) & y_{22 n}(s)
\end{array}\right], \\
& =j\left[\begin{array}{cc}
0 & K_{\infty} \\
K_{\infty} & 0
\end{array}\right]+\sum_{k=1}^{N} \frac{1}{s-j \lambda_{k}}\left[\begin{array}{ll}
r_{11 k} & r_{12 k} \\
r_{21 k} & r_{22 k}
\end{array}\right],
\end{aligned}
$$

where the real constant $K_{\infty}=0$, except for the fully canonical case where the nominator and denominator polynomials of the Y-parameters in the admittance matrix are of the same order, i.e. $n_{f z}=N$.

To determine $K_{\infty}$ for the fully canonical case it is convenient to evaluated its value at $s=j \infty$,

$$
j K_{\infty}=\left.\frac{y_{21 n}(s)}{y_{d}(s)}\right|_{s=j \infty}=\left.\frac{j P(s) / \epsilon}{y_{d}(s)}\right|_{s=j \infty},
$$

where the constant $K_{\infty}$ is independent of $s$. Due to the process followed to build up $y_{d}(s)$, its highest degree coefficient has a value of

$$
y_{d}(j \infty)=e_{N}+f_{N}=1+\frac{1}{\epsilon_{R}}
$$

and since the highest degree coefficient of polynomial $P(s)$ is normalised, $P(j \infty)=1$, it results in

$$
K_{\infty}=\frac{1 / \epsilon}{1+1 / \epsilon_{R}}
$$

\section{Case 2 - Synthesis of the Y-parameter matrix $\left[Y_{N}\right]$ using a circuit approach}

The $k$-th resonator in Fig. 3.22 (a) can be represented by an $A B C D$ transfer matrix by cascading the elements in Fig. 3.22 (b) as follows

$$
\begin{aligned}
{[A B C D]_{k} } & =\left[\begin{array}{cc}
0 & j / M_{S k} \\
j M_{S k} & 0
\end{array}\right]\left[\begin{array}{cc}
1 & 0 \\
j B_{k}+C_{k} s & 1
\end{array}\right]\left[\begin{array}{cc}
0 & j / M_{L k} \\
j M_{L k} & 0
\end{array}\right], \\
& =-\left[\begin{array}{cc}
\frac{M_{L k}}{M_{S k}} & \frac{s C_{k}+j B_{k}}{M_{S k} M_{L k}} \\
0 & \frac{M_{S k}}{M_{L k}}
\end{array}\right],
\end{aligned}
$$

where $M_{S k}$ and $M_{L k}$ are the source-resonator and load-resonator admittance inverters respectively. The $A B C D$-parameters of Eq. (3.5.46) are converted to the equivalent 
Y-parameter matrix producing Eq. (3.5.47).

$$
\begin{aligned}
{\left[Y_{k}\right] } & =\left[\begin{array}{ll}
y_{11 k}(s) & y_{12 k}(s) \\
y_{21 k}(s) & y_{22 k}(s)
\end{array}\right] \\
& =\frac{1}{s C_{k}+j B_{k}}\left[\begin{array}{cc}
M_{S k}^{2} & M_{S k} M_{L k} \\
M_{S k} M_{L k} & M_{L k}^{2}
\end{array}\right]
\end{aligned}
$$

The fully canonical two-port admittance matrix $\left[Y_{N}\right]$ for the parallel-connected transverse array (as in Fig. 3.22) is the sum of the Y-parameter matrices for the $N$ individual sections. The Y-parameter matrix $\left[Y_{S L}\right]$ for the direct source-load coupling matrix $M_{S L}$ is added [29], to produce

$$
\begin{aligned}
{\left[Y_{N}\right] } & =\left[\begin{array}{cc}
y_{11}(s) & y_{12}(s) \\
y_{21}(s) & y_{22}(s)
\end{array}\right], \\
& =\left[Y_{S L}\right]+\sum_{k=1}^{N}\left[Y_{k}\right] \\
& =j\left[\begin{array}{cc}
0 & M_{S L} \\
M_{S L} & 0
\end{array}\right]+\sum_{k=1}^{N} \frac{1}{s C_{k}+j B_{k}}\left[\begin{array}{cc}
M_{S k}^{2} & M_{S k} M_{L k} \\
M_{S k} M_{L k} & M_{L k}^{2}
\end{array}\right] .
\end{aligned}
$$

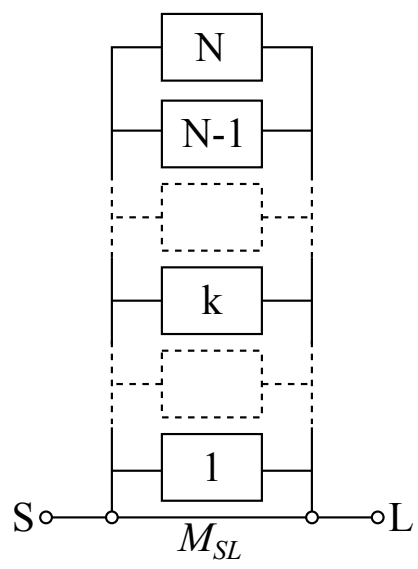

(a)

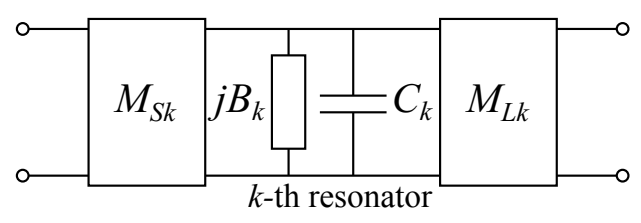

(b)

Figure 3.22: Canonical transversal array. (a) $N$-resonator transversal array including direct source-load coupling $M_{S L}$. (b) Equivalent circuit of the $k$-th resonator with coupling in the transversal array.

The two expressions for $\left[Y_{N}\right]$, Eqs. (3.5.42) and (3.5.48), are equated producing the following relationship between the residues and the transversal coupling matrix elements

$$
\begin{array}{lll}
M_{S L}=K_{\infty}, & \\
C_{k}=1 & \text { and } & B_{k}=-\lambda_{k}\left(\equiv M_{k k}\right), \\
M_{L k}^{2}=r_{22 k} & \text { and } & M_{S k} M_{L k}=r_{21 k} .
\end{array}
$$

The capacitors $C_{k}$ of the parallel networks are all unity and the frequency-invariant susceptances $B_{k}$ represent the self couplings $M_{11}$ to $M_{N N}$ [27],

$$
M_{L k}=\sqrt{r_{22 k}},
$$


and

$$
M_{S k}=\frac{r_{21 k}}{\sqrt{r_{22 k}}}=\sqrt{r_{11 k}} \quad k=1,2, \cdots, N .
$$

From Eqs. (3.5.49) to (3.5.51) the reciprocal $N+2$ transversal coupling matrix $\mathbf{M}$ is constructed. As shown by Fig. 3.23, $M_{S k}$ and $M_{L k}$ are the $N$ input and output couplings and occupy the first and last row and column of the matrix from 1 to $N$ respectively. All other entries are zero [27].

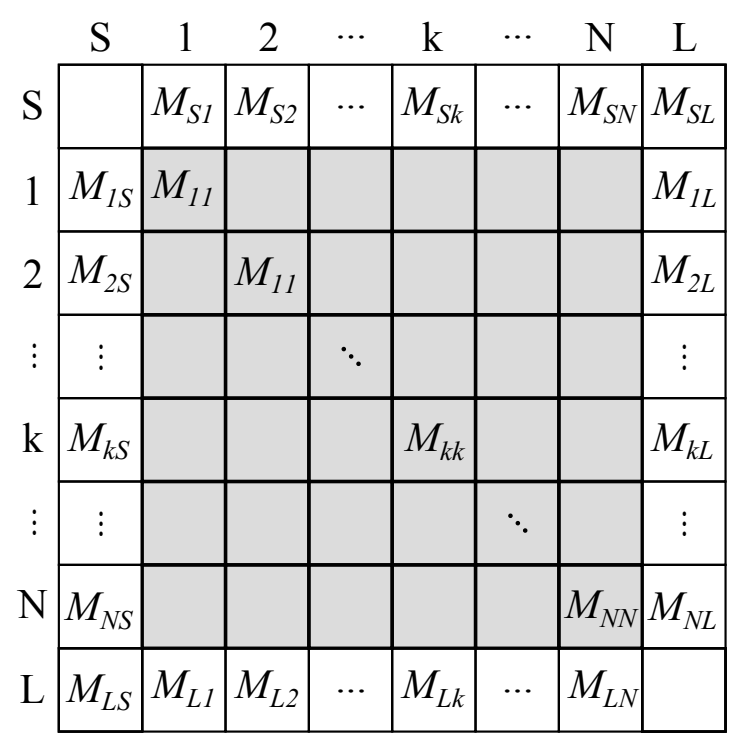

Figure 3.23: $N+2$ canonical coupling matrix $\mathbf{M}$ for the transversal array. The matrix is symmetric about the principal diagonal, i.e., $M_{i j}=M_{j i}$.

A MATLAB script (provided in Appendix B.2) generates the transversal coupling matrix M (see Table 3.2) from the IF filter's S-parameters as calculated in section 3.5.4.

Table 3.2: The transversal array's $N+2$ canonical coupling matrix for the IF filter

\begin{tabular}{|c||c|c|c|c||c|}
\hline 0 & 0.3553 & -0.5574 & 0.5574 & -0.3553 & 0 \\
\hline \hline 0.3553 & 1.2005 & 0 & 0 & 0 & 0.3553 \\
\hline-0.5574 & 0 & 0.5849 & 0 & 0 & 0.5574 \\
\hline 0.5574 & 0 & 0 & -0.5849 & 0 & 0.5574 \\
\hline-0.3553 & 0 & 0 & 0 & -1.2005 & 0.3553 \\
\hline \hline 0 & 0.3553 & 0.5574 & 0.5574 & 0.3553 & 0 \\
\hline
\end{tabular}

The elements of the transversal coupling matrix (in Table 3.2) may be realised directly by the coupling elements of a filtering structure. However, the multiple source and load couplings of the transversal array is not ideal for lumped element filter implementations. The negative entries on the coupling matrix are inductive elements when converted to passive lumped structures. The use of few coupling inductors with small values is desired. At the IF filter's low operating frequency inductors tend to have large values. In practice the manufacturing tolerances on large inductors are high and they usually have relatively low quality factors. Resulting in more laborious tuning, greater insertion loss, and the selfcoupling elements on the diagonal of the $\mathbf{M}$ matrix require more adjustment. Similarity 
transforms (sometimes called rotations) are applied to produce a more convenient coupling topology [27].

The use of similarity transforms ensure that the eigenvalues and eigenvectors of the matrix $\mathbf{M}$ are preserved. Consequently the transformed matrix will yield the same transfer and reflection characteristics as the original matrix [27].

\section{Similarity transformation and matrix reconfiguration}

A similarity transform is applied to a $N \times N$ coupling matrix $\mathbf{M}_{r}$ by pre-multiplying $\mathbf{M}_{r}$ by a $N \times N$ rotation matrix $\mathbf{R}_{r}$ and post-multiplying $\mathbf{M}_{r}$ by the transpose of $\mathbf{R}_{r}$,

$$
\mathbf{M}_{r}=\mathbf{R}_{r} \mathbf{M}_{r-1} \mathbf{R}_{r}^{t} \quad r=1,2,3, \cdots, R .
$$

The resultant matrix $\mathbf{M}_{R}$ at the end of the series of transformations yields the same performance as the original matrix $\mathbf{M}_{0}[27]$.

The rotation matrix is constructed as illustrated by the example in Fig. 3.24. Selecting a pivot of $[i, j](i \neq j)$ for matrix $\mathbf{R}_{r}$ means that elements $R_{i i}=R_{j j}=\cos \theta_{r}, R_{j i}=-R_{i j}=$ $\sin \theta_{r}$, where $i, j \neq \mathrm{S}$ or $\mathrm{L}$, and $\theta_{r}$ is the angle of rotation. The remaining principal diagonal entries are equal to 1 and all other undefined off-diagonal entries are zero.

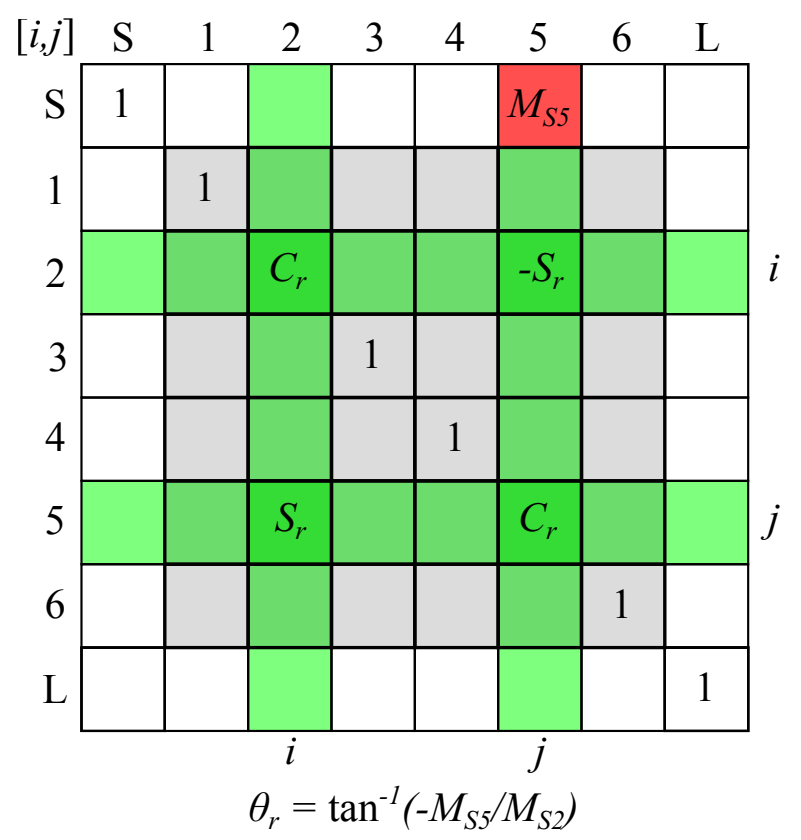

Figure 3.24: Example of a sixth degree rotational matrix $\mathbf{R}_{r}$ with pivot point $[2,5]$. The angle $\theta_{r}$ is setup to annihilate entry $M_{S 5}$.

If a similarity transform with pivot $[i, j]$ and angle $\theta_{r} \neq 0$ is applied to a coupling matrix $\mathbf{M}_{r-1}$ as in Eq. (3.5.52). The elements in rows and columns $i$ and $j$ of the resultant matrix $\mathbf{M}_{r}$ are transformed from the corresponding elements in $\mathbf{M}_{r-1}$. These elements are in line with the pivot's crossings (and are indicated in green on Fig. 3.24). For the $k$ th element not at the pivot cross-points (i.e., $k \neq i, j$ ) in row or column $i$ or $j$ the value is

$$
\begin{array}{ll}
M_{i k, r}=c_{r} M_{i k, r-1}-s_{r} M_{j k, r-1} & \text { for an element in row } i, \\
M_{j k, r}=s_{r} M_{i k, r-1}+c_{r} M_{j k, r-1} & \text { for an element in row } j, \\
M_{k i, r}=c_{r} M_{k i, r-1}-s_{r} M_{k j, r-1} & \text { for an element in column } i, \\
M_{k j, r}=s_{r} M_{k i, r-1}+c_{r} M_{k j, r-1} & \text { for an element in column } j,
\end{array}
$$


where $k(\neq i, j)=1,2,3, \cdots, N, c_{r}=\cos \theta_{r}$, and $s_{r}=\sin \theta_{r}$. The elements on the cross-points of the pivot $\left(M_{i i}, M_{j j}, M_{i j}=M_{j i}\right)$ of $\mathbf{M}_{r}$ are

$$
\begin{aligned}
M_{i i, r} & =c_{r}^{2} M_{i i, r-1}-2 s_{r} c_{r} M_{i j, r-1}+s_{r}^{2} M_{j j, r-1}, \\
M_{j j, r} & =s_{r}^{2} M_{i i, r-1}+2 s_{r} c_{r} M_{i j, r-1}+c_{r}^{2} M_{j j, r-1}, \\
M_{i j, r} & =M_{i j, r-1}\left(c_{r}^{2}-s_{r}^{2}\right)+s_{r} c_{r}\left(M_{i i, r-1}-M_{j j, r-1}\right) .
\end{aligned}
$$

During the matrix reduction process two properties of the similarity transform is exploited:

- In matrix $\mathbf{M}_{r}$ only the elements on rows and columns $i$ and $j$ related to the pivot $[i, j]$ of $\mathbf{R}_{r}$ are affected by the transform (if $\theta_{r} \neq 0$ ).

- Assuming two zero elements face each other across the rows and columns of the pivot before the transform, these values remain zero after the transform.

The angle $\theta_{r}$ needed to annihilate (i.e. null) an entry in matrix $\mathbf{M}_{r-1}$ is determined by setting the values related to matrix $\mathbf{M}_{r}$ to zero in Eqs. (3.5.53) and (3.5.54), then solving for $\theta_{r}$

$$
\begin{aligned}
& \theta_{r}=\arctan \left(\frac{M_{i k}}{M_{j k}}\right) \quad \text { for the } k \text { th element in row } i\left(M_{i k}\right), \\
& \theta_{r}=-\arctan \left(\frac{M_{j k}}{M_{i k}}\right) \quad \text { for the } k \text { th element in row } j\left(M_{j k}\right), \\
& \theta_{r}=\arctan \left(\frac{M_{k i}}{M_{k j}}\right) \quad \text { for the } k \text { th element in column } i\left(M_{k i}\right), \\
& \theta_{r}=-\arctan \left(\frac{M_{k j}}{M_{k i}}\right) \quad \text { for the } k \text { th element in column } j\left(M_{k j}\right) .
\end{aligned}
$$

The angle $\left(\theta_{r}\right)$ of rotation for the cross-pivot entries $\left(M_{i i}, M_{j j}, M_{i j}, M_{j i}\right)$ are documented in [28]. These transforms are not applied during the transversal matrix $\mathbf{M}$ transformation presented in Table 3.3 .

Table 3.3: Similarity transform sequence for the reduction of the transversal matrix

\begin{tabular}{c|c|c|c|c|c|c|c}
\hline \hline \multirow{2}{*}{$\begin{array}{c}\text { Transform } \\
\text { Number } \\
\mathrm{r}\end{array}$} & \multirow{2}{*}{ Pivot $[i, j]$} & \multirow{2}{*}{$\begin{array}{c}\text { Elements } \\
\text { to be }\end{array}$} & \multicolumn{4}{|c}{$\theta_{r}=\tan ^{-1}\left(c M_{k l} / M_{m n}\right)$} \\
\cline { 4 - 8 } & & Annihilated & $k$ & $l$ & $m$ & $n$ & $c$ \\
\hline \hline 1 & {$[1,2]$} & $M_{S 2}\left(\right.$ and $\left.M_{2 S}\right)$, row $S$ & $\mathrm{~S}$ & 2 & $\mathrm{~S}$ & 1 & -1 \\
2 & {$[1,3]$} & $M_{S 3}\left(\right.$ and $\left.M_{3 S}\right)$, row $S$ & $\mathrm{~S}$ & 3 & $\mathrm{~S}$ & 1 & -1 \\
3 & {$[1,4]$} & $M_{S 4}\left(\right.$ and $\left.M_{4 S}\right)$, row $S$ & $\mathrm{~S}$ & 4 & $\mathrm{~S}$ & 1 & -1 \\
\hline 4 & {$[2,4]$} & $M_{L 2}\left(\right.$ and $\left.M_{2 L}\right)$, column 2 & $\mathrm{~L}$ & 2 & $\mathrm{~L}$ & 4 & 1 \\
5 & {$[3,4]$} & $M_{L 3}\left(\right.$ and $\left.M_{3 L}\right)$, column 3 & $\mathrm{~L}$ & 3 & $\mathrm{~L}$ & 4 & 1 \\
\hline 6 & {$[3,2]$} & $M_{L 3}\left(\right.$ and $\left.M_{3 L}\right)$, column 2 & $\mathrm{~L}$ & 2 & $\mathrm{~L}$ & 3 & -1 \\
\hline \hline & {$[2,3]$} & $M_{13}\left(\right.$ and $\left.M_{31}\right)$, row 1 & 1 & 3 & 1 & 2 & -1 \\
\hline \hline
\end{tabular}

Due to symmetry in the outer rows and columns of the transversal matrix in Table 3.2 , corresponding entries in these rows and column are annihilated simultaneously. The pivots of the rotations to annihilate the source couplings start at position $[i=1, j=$ $2]$ and progress toward the end of the matrix until position $[i=1, j=4]$. The load 
couplings are nulled by moving the pivot from $[i=4, j=2]$ to $[i=4, j=3]$. An additional rotation is applied to transform inductive coupling elements between resonators to capacitive coupling. Element $M_{1,3}$ is annihilated because only two finite zero's are desired. Therefore, a third cross coupled element is redundant. The transforms are shown in sequence in Table 3.3 .

A MATLAB script provided in Appendix B.3 reduces the transversal matrix in Table 3.2, by following the steps shown in Table 3.3, to the matrix presented in Table 3.4.

Table 3.4: Transformed $N+2$ coupling matrix that has the desired topology and filter characteristics

\begin{tabular}{|c||c|c|c|c||c|}
\hline 0 & 0.9349 & 0 & 0 & 0 & 0 \\
\hline \hline 0.9349 & 0 & 0.8092 & 0 & -0.0692 & 0 \\
\hline 0 & 0.8092 & 0 & 0.6847 & 0 & 0 \\
\hline 0 & 0 & 0.6847 & 0 & 0.8092 & 0 \\
\hline 0 & -0.0692 & 0 & 0.8092 & 0 & 0.9349 \\
\hline \hline 0 & 0 & 0 & 0 & 0.9349 & 0 \\
\hline
\end{tabular}

\section{Passive lumped elements from a coupling matrix}

The coupled resonator filter is constructed using impedance inverters and LC resonators as shown in Fig. 3.25. In practice the inverters are either T-or П-networks made of frequency dependent reactive elements. The LC resonator's lumped elements can be connected in series or parallel.

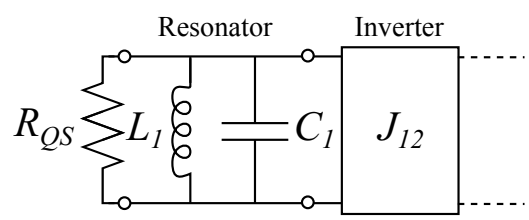

(a)

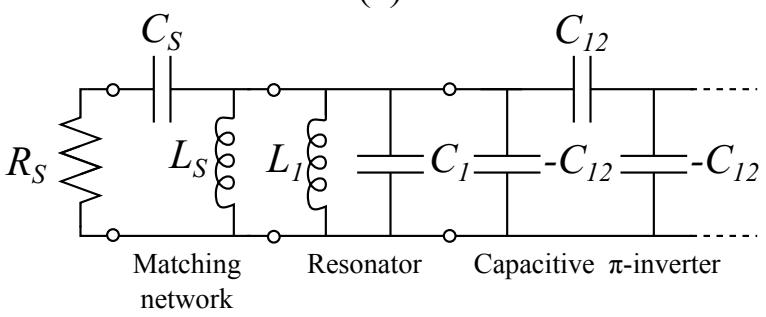

(b)

Figure 3.25: (a) Idealised filter elements illustrating the loaded Q source impedance $\left(R_{Q S}\right)$, LC-resonator, and the admittance inverter between resonator 1 and 2. (b) Practical filter elements, matching network between the loaded $\mathrm{Q}$ and desired input impedance $\left(R_{S}\right)$. A capacitive $\Pi$-inverter network replaces the ideal $J$-inverter.

The physical inverters are frequency dependent and only produce the correct inverterconstants at their centre frequencies. Therefore, the coupled resonator filter design is only suited to narrow band implementations. A relative bandwidth of $10 \%$ is acceptable when using lumped elements (inductors and capacitors) in a coupled resonator filter. 
The coupling matrix $\mathbf{M}$ is normalised according to the synthesised filter's $3 \mathrm{~dB}$ relative bandwidth. To undo this normalisation a new coupling matrix is defined as follows

$$
\mathbf{k}=\left(\frac{f_{2}-f_{S F C}}{f_{\text {centre }}}\right) \mathbf{M}=\left(\frac{\delta f_{-3 d B}}{f_{\text {centre }}}\right) \mathbf{M}=\Delta \mathbf{M} .
$$

Refer to Fig. 3.18 for an illustrative definition of the variables used in Eq. (3.5.56).

To keep the synthesis general, it is assumed that the IF filter's frequency response is asymmetrical. Therefore, the resonators have offset resonant frequencies. The self coupling coefficients $\left(M_{i i}\right)$ on the diagonal of the coupling matrix indicate the required adjustment. By analysing a parallel resonator and equating its impedance to the bandwidth scaled self coupling coefficient, the adjusted centre frequency is determined

$$
\begin{gathered}
y_{i}(s)=C_{i} s+\frac{1}{L_{i} s}, \\
y_{i}\left(j \omega_{0}\right)=\frac{1}{j M_{i i}}=C_{i} j \omega_{0}+\frac{1}{L_{i} j \omega_{0}},
\end{gathered}
$$

where $\omega_{0}=\omega_{\text {centre }}$ is the design centre frequency during the filter synthesis. At the compensated centre frequency $\omega_{0 i}$, the lossless resonator's admittance is zero, i.e. $y_{i}\left(j \omega_{0 i}\right)=0$. Consequently, the following relationship is found

$$
\omega_{0 i}=\frac{1}{\sqrt{L_{i} C_{i}}}
$$

and the characteristic impedance is

$$
Z_{i}=\sqrt{\frac{L_{i}}{C_{i}}} .
$$

By applying Eqs. (3.5.57) to (3.5.60) the resonator's compensated centre frequency is identified

$$
\begin{aligned}
\frac{M_{i i}}{Z_{i}} & =\frac{\omega_{0 i}}{\omega_{0}}-\frac{\omega_{0}}{\omega_{0 i}}, \\
\omega_{0 i} & =\omega_{0}\left[\frac{1}{2} \frac{M_{i i}}{Z_{i}}+\sqrt{\left(\frac{1}{2} \frac{M_{i i}}{Z_{i}}\right)^{2}+1}\right] .
\end{aligned}
$$

The values of the frequency compensated inductor and capacitor pair are determined using Eqs. (3.5.61) and (3.5.62)

$$
\begin{aligned}
C_{i} & =\frac{1}{Z_{i} \omega_{0 i}}, \\
L_{i} & =\frac{Z_{i}}{\omega_{0 i}} .
\end{aligned}
$$

The slope of the impedance/admittance function of a resonator is an important constant in coupled resonator filters because it represents the resonator's susceptance at its resonant frequency. For parallel resonators, as illustrated in Fig. 3.25, the normalised slope parameter at $\omega_{0 i}$ is given by

$$
\begin{aligned}
\beta_{i} & =\left.\frac{\omega_{0 i}}{2} \frac{d B_{i}}{d \omega}\right|_{\omega=\omega_{0 i}}, \\
& =\frac{\omega_{0 i}}{2} \frac{d}{d \omega}\left[C_{i} \omega-\frac{1}{L_{i} \omega}\right]_{\omega=\omega_{0 i}}, \\
& =\frac{C_{i} \omega_{0 i}}{2}+\frac{1}{2 L_{i} \omega_{0 i}} .
\end{aligned}
$$


At the resonators resonant frequency its impedance $y_{i}\left(j \omega_{0 i}\right)=0$, therefore, from Eq. (3.5.57) it is evident that

$$
C_{i} \omega_{0 i}=\frac{1}{L_{i} \omega_{0 i}} .
$$

Consequently, the admittance slope parameter $\beta_{i}$ of the $i$-th resonator is calculated

$$
\beta_{i}=C_{i} \omega_{0 i}=\frac{1}{L_{i} \omega_{0 i}} .
$$

The admittance inverter coefficients $\left(J_{i j}\right)$ are scaled according to the neighbouring resonator's susceptances as follows

$$
J_{i j}=\sqrt{\beta_{i} \beta_{j}} k_{i j}
$$

where $k_{i j}$ are the coefficients of the frequency scaled coupling matrix $\mathbf{k}$.

By calculating the loaded Q-factor $\left(Q_{L}\right)$ for the filter's end resonators it is possible to determine the required source and load matching impedances. A parallel resonator's external quality factor is calculated by [8]

$$
Q_{e}=\frac{\beta_{S}}{G_{S}} .
$$

The source conductance $\left(G_{S}=\frac{1}{R_{S}}\right)$, as seen from resonator one through the admittance inverter $J_{S 1}$, is taken into account

$$
Q_{e}=\frac{\beta_{S}}{J_{S 1}^{2} / G_{Q S}} .
$$

Applying Eq. (3.5.66) to Eq. (3.5.68) produces the external Q-factor

$$
\begin{aligned}
Q_{e} & =\frac{G_{Q S}}{\beta_{1} k_{S 1}^{2}}, \\
& =\frac{G_{Q S}}{\beta_{1} \Delta^{2} M_{S 1}^{2}} .
\end{aligned}
$$

The loaded Q-factor is related to the bandwidth by

$$
Q_{L}=\frac{\omega_{0 i}}{\delta f_{-3 d B}}=\frac{1}{\Delta}
$$

The unloaded Q-factor $\left(Q_{u}\right)$ for the parallel resonator is infinite when assuming the resonators are lossless because no energy is dissipated in the resonator [8] [28]. The loaded $\mathrm{Q}$ is expressed in terms of the external and unloaded $\mathrm{Q}$ by

$$
\frac{1}{Q_{L}}=\frac{1}{Q_{e}}+\frac{1}{Q_{u}}
$$

where $Q_{L} \approx Q_{e}$ for the lossless resonator. The source resistance seen at the input to the filter is determined

$$
Q_{L}=\frac{1}{\Delta} \approx Q_{e}=\frac{G_{Q S}}{\beta_{1} \Delta^{2} M_{S 1}^{2}},
$$

solving for $G_{Q S}$, where $G_{Q S}=\frac{1}{R_{Q S}}$ results in

$$
R_{Q S}=\frac{1}{\beta_{1} \Delta M_{S 1}^{2}}
$$


The same holds at the filter's output, producing

$$
R_{Q L}=\frac{1}{\beta_{N} \Delta M_{N L}^{2}} .
$$

The loaded Q input and output impedances, calculated by Eqs. (3.5.73) and (3.5.74), are matched to the desired characteristic impedance $\left(Z_{0}\right)$ by narrowband LC matching networks as shown in Fig. 3.25.

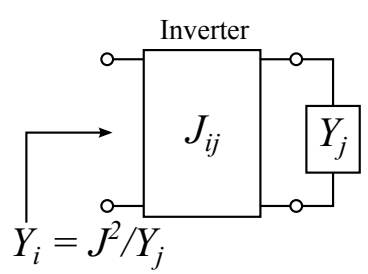

(a)

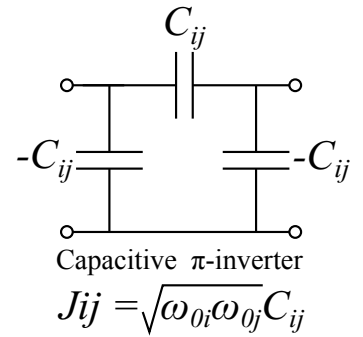

(b)

Figure 3.26: (a) Operation of the admittance inverter. (b) Implementation of the admittance inverter using a capacitor network.

Capacitive П-inverter networks are chosen, because the inverter's negative shunt capacitors simply add to the adjacent parallel resonator capacitors. In practice, coupling capacitors are more convenient than inductors, because of component tolerances, quality factors, and availability.

The capacitor values of the П-inverter shown in Fig. 3.26 are calculated by [8],

$$
C_{i j}=\frac{J_{i j}}{\sqrt{\omega_{0 i} \omega_{0 j}}}
$$

In cases where the cross coupling coefficients of matrix $\mathbf{M}$ are negative, it results in negative coupling capacitor $C_{i j}$ values. Therefore, the $\Pi$-inverter's series capacitor is transformed to an inductor by ensuring both components have equal impedance,

$$
\begin{aligned}
X_{C}=j \sqrt{\omega_{0 i} \omega_{0 j}} C_{i j} & =\frac{1}{j \sqrt{\omega_{0 i} \omega_{0 j}} L_{i j}}=X_{L}, \\
L_{i j} & =\frac{-1}{\omega_{0 i} \omega_{0 j} C_{i j}} .
\end{aligned}
$$

A MATLAB script, provided in Appendix B.4, calculates the lumped element values for the compensated LC resonators, input and output loaded Q impedances, source load matching networks, and the capacitive $\Pi$-inverters. The appropriate elements are combined to form the IF filter network shown in Fig. 3.27 (c) and the element values are given in Table 3.5. 


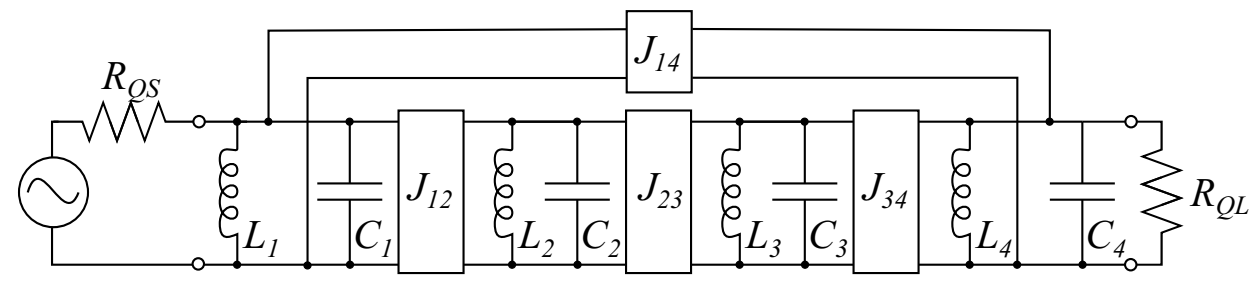

(a)

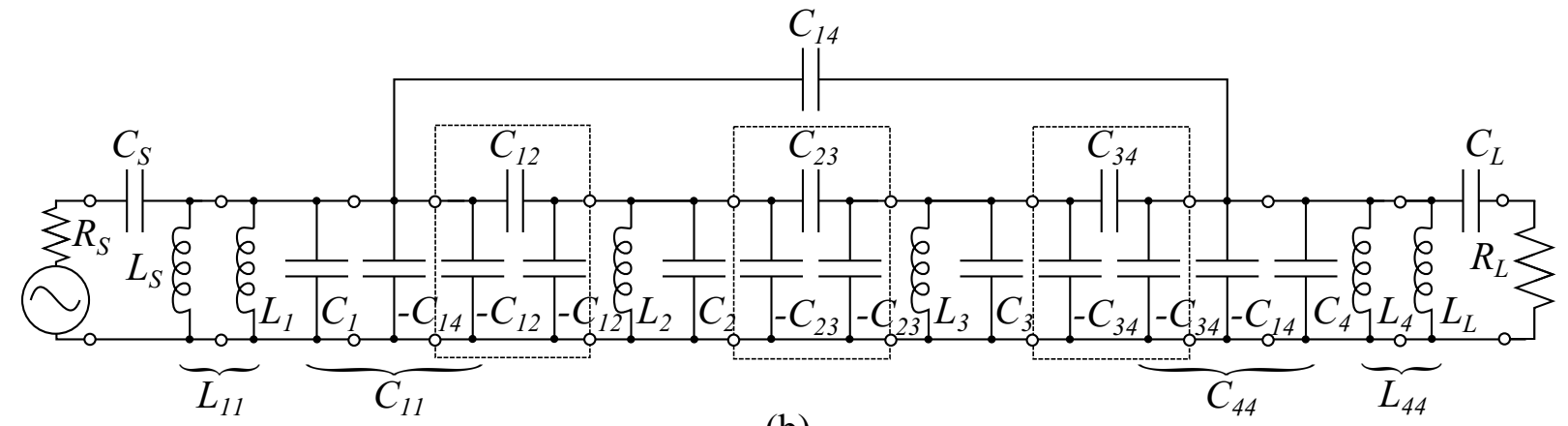

(b)

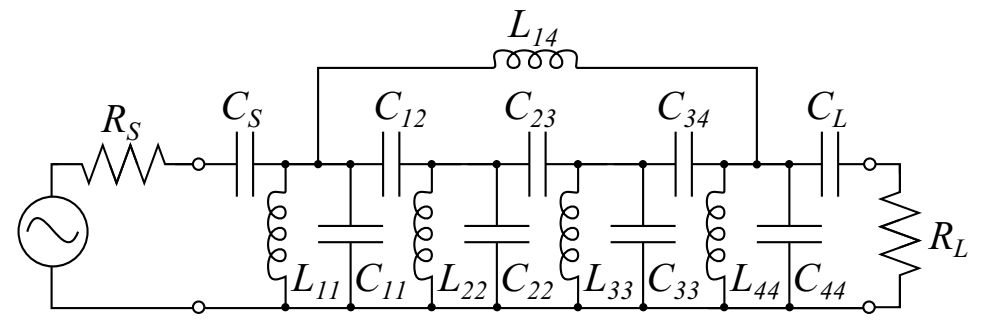

(c)

Figure 3.27: (a) IF filter lumped element circuit with ideal admittance inverters, (b) equivalent lumped element model and $\Pi$-inverters, (c) practical IF filter implementation.

Table 3.5: IF filter lumped element values

\begin{tabular}{c|c|c}
\hline \hline Elements & Values & Units \\
\hline$R_{S}, R_{L}$ & 50 & $\Omega$ \\
\hline$C_{S}, C_{L}$ & 720 & $\mathrm{pF}$ \\
$C_{11}, C_{44}$ & 2.9 & $\mathrm{nF}$ \\
$C_{22}, C_{33}$ & 2.7 & $\mathrm{nF}$ \\
$C_{12}, C_{34}$ & 150 & $\mathrm{pF}$ \\
$C_{23}$ & 130 & $\mathrm{pF}$ \\
\hline$L_{11}, L_{44}$ & 6.1 & $\mu \mathrm{H}$ \\
$L_{22}, L_{33}$ & 7.5 & $\mu \mathrm{H}$ \\
$L_{14}$ & 1.7 & $\mathrm{mH}$ \\
\hline
\end{tabular}

Note that a zero-IF SFC is usually a simple second order high pass filter. The baseband filter is less complex and requires fewer components than the IF filter. This should be taken into account when considering an IF FMCW radar receiver.

\subsubsection{Measured intermediate frequency filter}

The IF filter's measured and simulated results are shown in Figs. 3.28 and 3.29. Compared to the synthesised filter response in Fig. 3.20, the measured and simulated lumped 
element frequency responses are asymmetrical. The asymmetry is due to the narrowband approximation of the negative cross coupling between resonator one and four $\left(J_{14}\right)$. The approximation, described by Eq. (3.5.76), is only accurate around the filter's centre frequency.

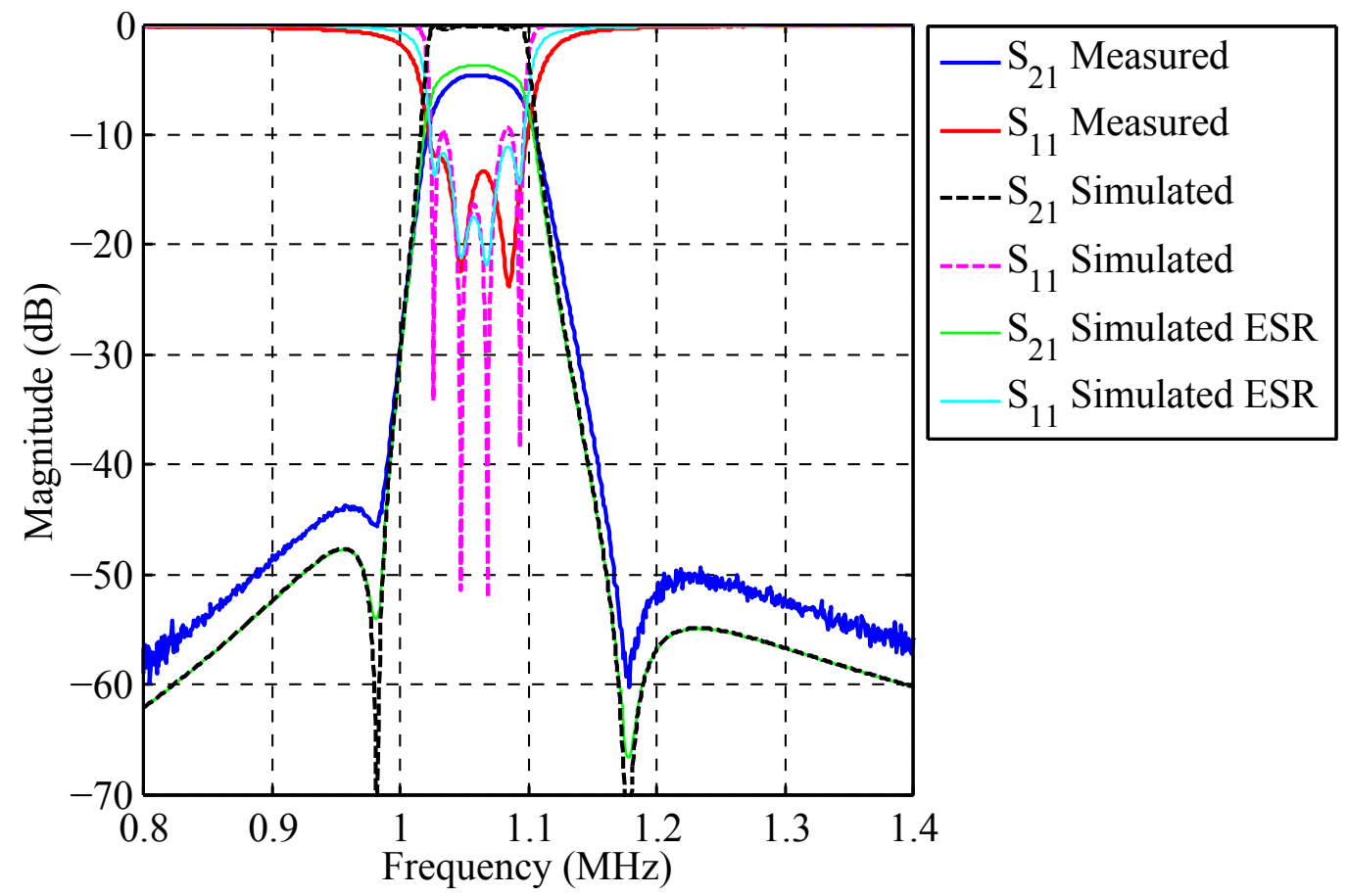

Figure 3.28: The IF filter's amplitude response. Shown are the measured results, ideal lumped element simulation, and ESR simulation.

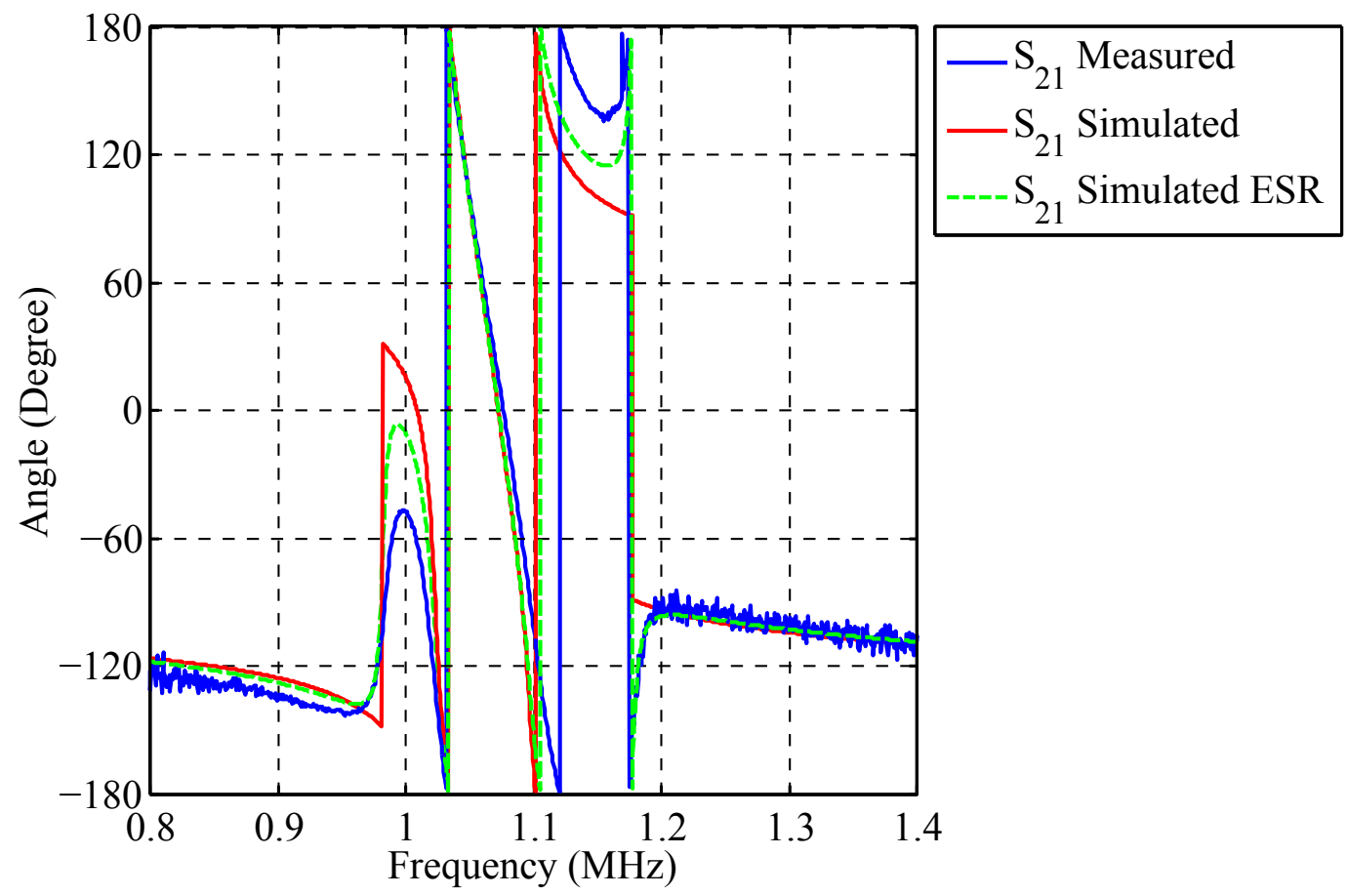

Figure 3.29: The IF filter's phase response. Shown are the measured results, the ideal lumped element simulation, and the ESR simulation. 
The ESR of each component used in the filter is calculated from its quality factor. The component Q-factors are found on data sheets and from measurements made using a network analyser. By including each component's ESR, a more accurate simulation is obtained as seen in Figs. 3.28 and 3.29.

The filter's resonators are constructed using single passive toroidal inductors. An inductor's Q-factor usually improves with less turns on the ferrite core. Therefore, the filter's insertion loss can be improved by replacing a single large inductor with multiple series inductors that have higher Q-factors.

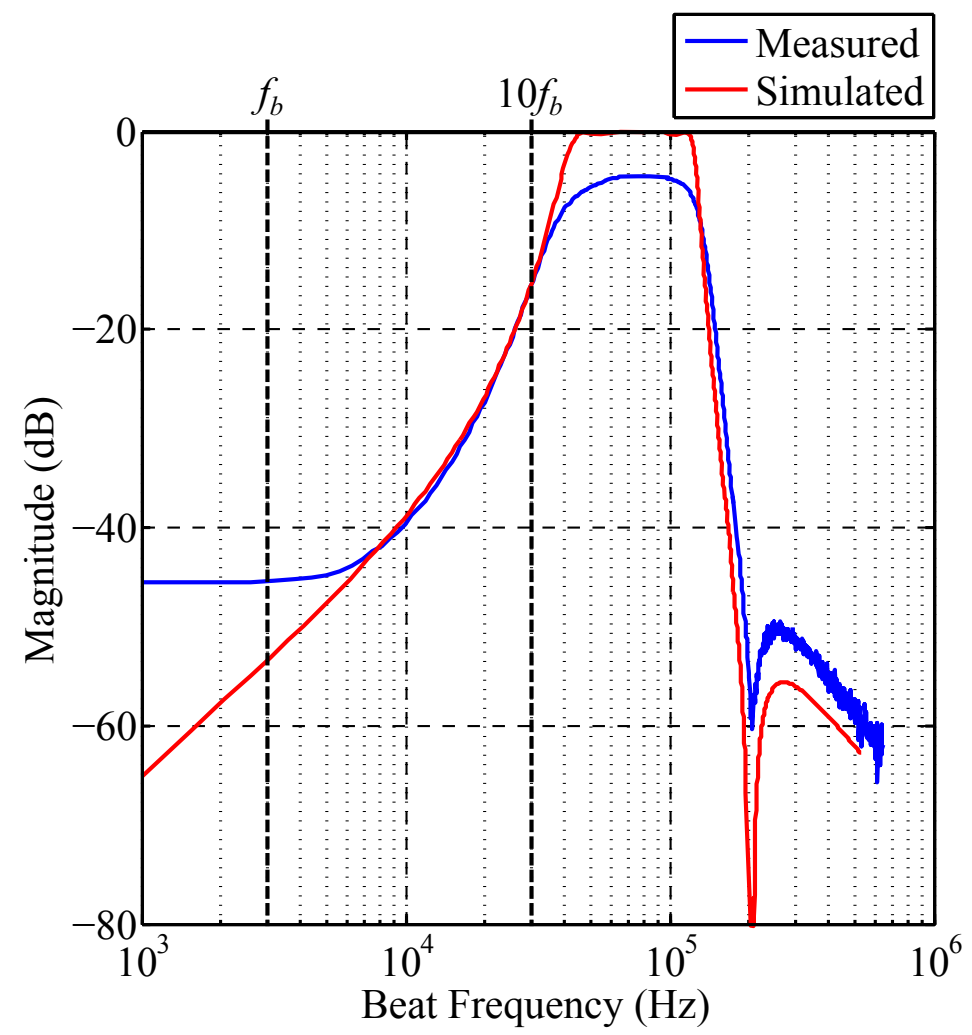

Figure 3.30: The IF filter response is transposed to baseband, where zero beat frequency is located at zero frequency.

Figure 3.30 is a transposed version of the simulated IF filter's frequency response. The $40 \mathrm{~dB} /$ decade baseband roll-off is illustrated by transposing the IF filter's zero beat frequency to zero frequency (x-axis origin). In other words the IF receiver is converted to baseband, where frequency is directly related to beat frequency. In Fig. 3.30 a roll-off of $37 \mathrm{~dB}$ is seen between $3 \mathrm{kHz}$ and $30 \mathrm{kHz}$ for the simulated response. Due to the measured filter's insertion loss, the filter roll-off plateaus at beat frequencies below $8 \mathrm{kHz}$.

\subsection{Anti-aliasing filter}

As illustrated in Figs. 2.1 and 2.12 a bandpass filter is added after the high voltage gain op-amps to prevent aliasing. Aliasing of harmonics and IMPs can occur because of strong reflections that cause the op-amps to saturate.

The anti-aliasing filter's requirements are fixed by applying the theory documented in section 3.4. The anti-aliasing filter is designed to operate in the third Nyquist zone. The filter spans from the start of the lower transition band (at $2 f_{s}-f_{0}=665 \mathrm{kHz}$ ) to 
the end of the upper transition band (at $3 f_{s}-f_{1}=1.39 \mathrm{MHz}$ ) as illustrated by Fig. 3.16. To prevent aliasing $60 \mathrm{~dB}$ attenuation is desired outside these frequency bounds in neighbouring Nyquist zones.

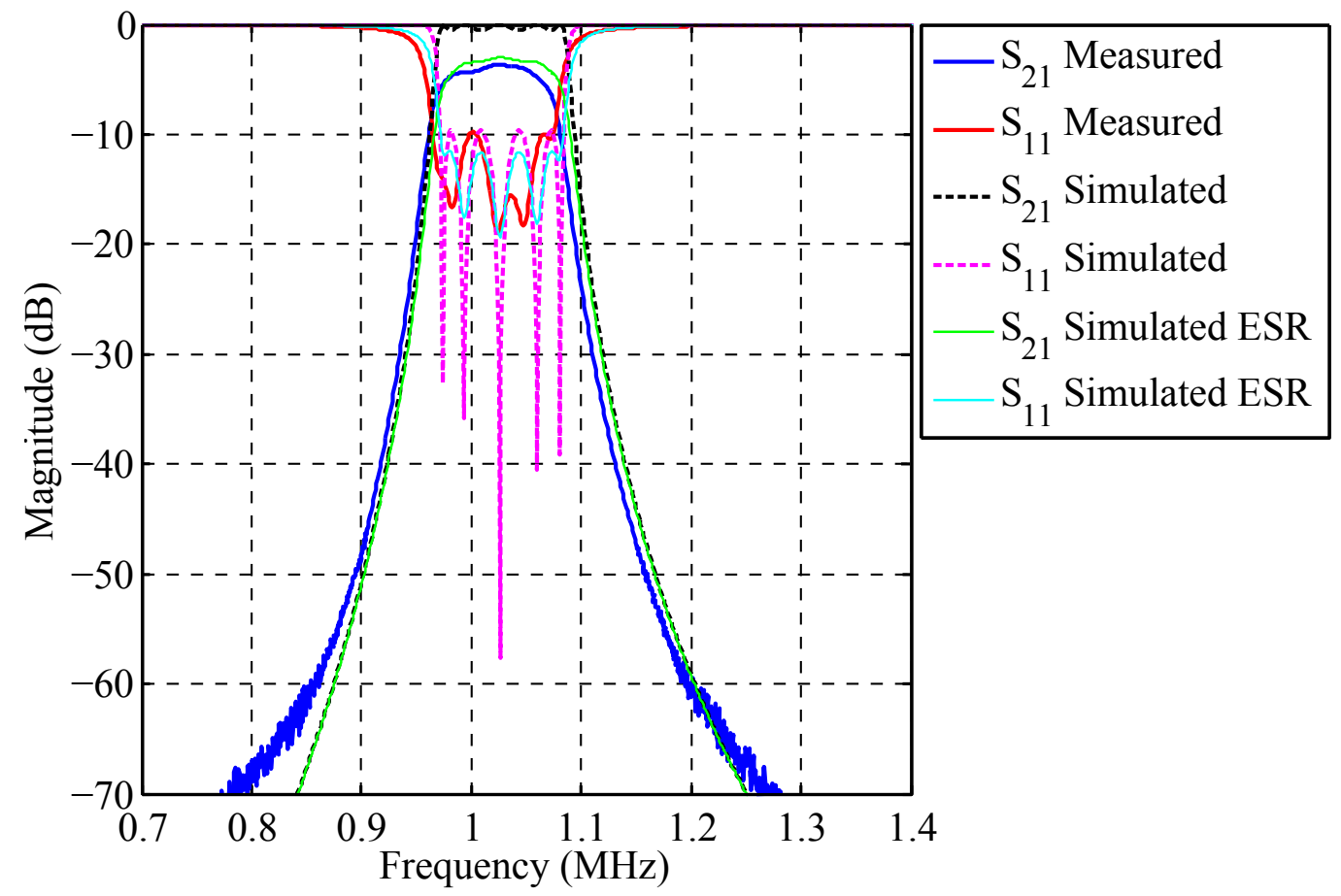

Figure 3.31: The anti-aliasing filter's amplitude response: measured results, ideal lumped element simulation, and ESR simulation.

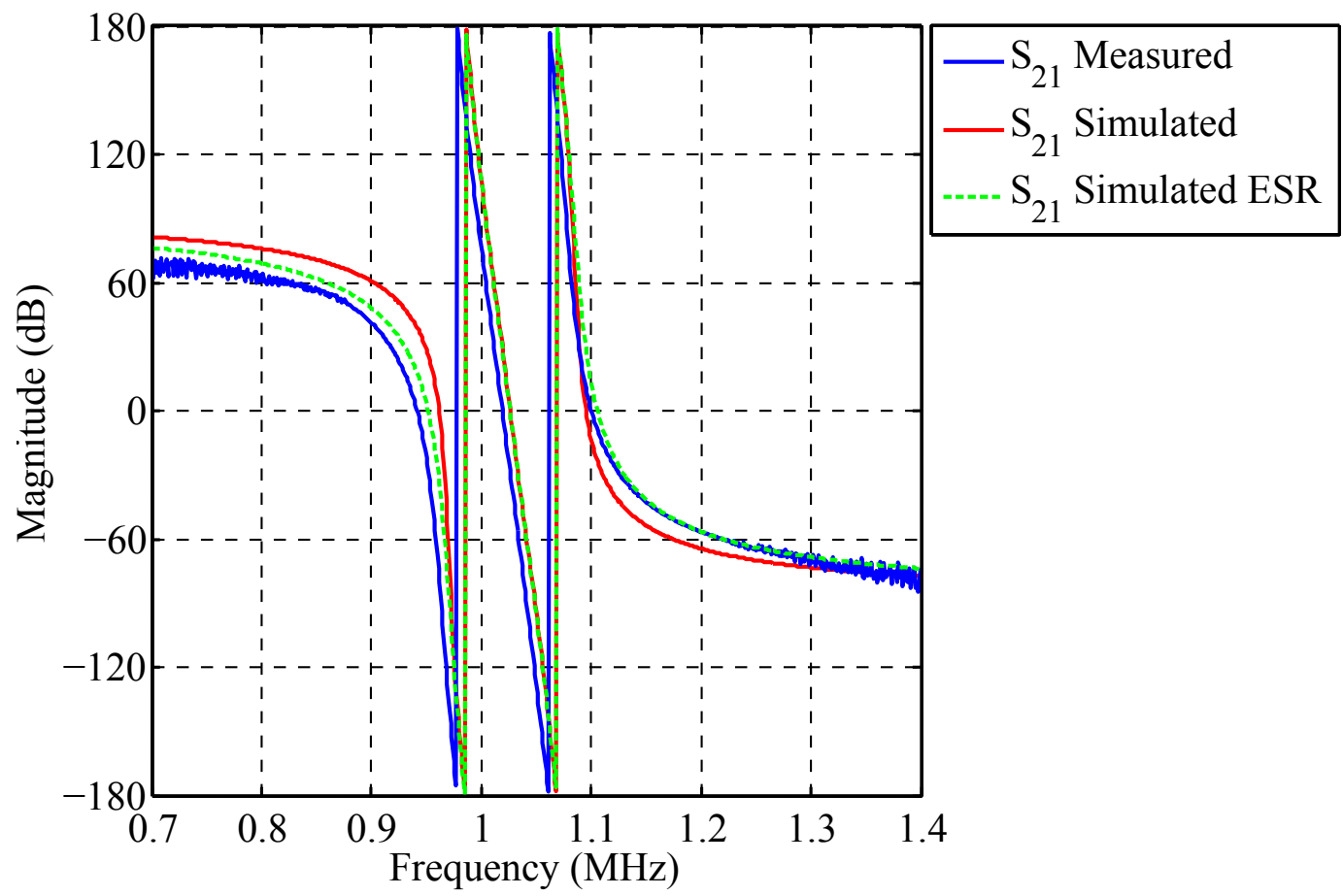

Figure 3.32: The anti-aliasing filter's phase response: measured results, ideal lumped element simulation, and ESR simulation. 
A fifth order Chebyshev bandpass filter is designed from element values documented in [8]. By applying the band-pass transform on the equal-ripple (Chebyshev) low-pass filter prototype the desired response is achieved. The anti-aliasing filter's schematic is provided in Fig. F.3 under Appendix F.1. The measured and simulated results are shown in Figs. 3.31 and 3.32.

\subsection{Conclusion}

In this chapter the receiver and image rejection topologies are discussed. The Hartley (or half complex) image rejection architecture is selected for its low complexity and because its design requires few components.

The Nyquist-Shannon sampling theorem and the design interaction between the ADC and anti-aliasing filter is discussed. This chapter also elaborates on the application of undersampling and the effect it has on the minimum required sample frequency.

An IF filter is designed for a dual purpose as an initial anti-aliasing filter and SFC. Large reflections from targets close to the radar are suppressed by the IF filter (SFC) and harmonics and IMPs generated by receiver saturation are attenuated by a second anti-aliasing filter.

Designing for SFC at an IF is more challenging than at baseband. The zero-IF SFC is usually a second order high pass filter. Therefore, the baseband SFC is less complex and requires fewer components than the IF filter.

The receiver's schematic and printed circuit board (PCB) layout is presented in Appendix F. 


\section{Chapter 4}

\section{Receiver noise}

An analysis of the FMCW radar's receiver noise is presented in this chapter. This includes a general discussion on noise and the impact noise has on RF system performance.

The IEEE Standard Dictionary of Electrical and Electronic Terms, defines noise as, unwanted disturbances superposed upon a useful signal that tend to obscure its information content [31]. This definition applies to both intrinsic and extrinsic noise. Where intrinsic noise refers to noise generated inside a system, while extrinsic noise describes noise originating elsewhere [32] [33].

\subsection{Overview}

This section gives a broad overview of noise, its origin, and the impact noise has on circuit operation and performance. In Appendix $\mathrm{C}$ some general guidelines are given on the notation used in this chapter.

Noise has a direct impact on the performance of $\mathrm{RF}$ and microwave systems: in most cases a receiver's noise floor determines the threshold of the minimum signal it can reliably detect. Noise is introduced to RF receivers from external environmental sources (extrinsic noise) and is generated internally (intrinsic noise) by the receiver circuitry [33]. Noise can be described in terms of noise temperature, noise figure or an equivalent noise voltage [8].

If the total cascaded noise figure of a receiver is known it is possible to determine the receiver's minimum detectable signal (MDS) level, which is used in quantifying a receiver's dynamic range (DR).

\subsubsection{Noise power}

In this chapter it is assumed that all noise signals have the property of being stochastic, band limited, and centred around a stationary frequency, $f_{0}$, with a random amplitude and phase, i.e.

$$
x(t)=A(t) e^{j\left(2 \pi f_{0} t+\phi(t)\right)} .
$$

Equation (4.1.1) describes a general stochastic noise signal, in which $x(t)$ is the instantaneous voltage across or current through a unit resistor $(1 \Omega)$. Due to this definition, $x^{2}(t)$ is referred to as the instantaneous power in the signal $x(t)$ [34]. The random and ergodic nature of a noise signal results in its mean value being zero. It is possible to calculate a noise signal's power spectral density (PSD) by taking the Fourier transform of the noise 
signal's auto-correlation, shown by Eqs. (4.1.2) and (4.1.3).

$$
\begin{gathered}
R_{x x^{*}}(\tau)=\lim _{T \rightarrow \infty} \frac{1}{2 T} \int_{-T}^{T} x(t+\tau) x^{*}(t) d t \\
S_{x x^{*}}(\omega) \stackrel{\mathrm{FT}}{\longleftrightarrow} R_{x x^{*}}(\tau)
\end{gathered}
$$

Signal $x(t)$ is assumed to be wide sense stationary (WSS), thus $R_{x x^{*}}(\tau)$ is time invariant [34]. The integral of the power spectral density $S_{x x^{*}}(\omega)$, is the expected instantaneous power of the signal $x(t)$ as shown below [34],

$$
\begin{aligned}
\frac{1}{2 \pi} \int_{-\infty}^{\infty} S_{x x^{*}}(\omega) d \omega & =\frac{1}{2 \pi} \int_{-\infty}^{\infty} \int_{-\infty}^{\infty} R_{x x^{*}}(\tau) e^{-j \omega \tau} d \tau d \omega \\
& =\int_{-\infty}^{\infty} R_{x x^{*}}(\tau)\left(\frac{1}{2 \pi} \int_{-\infty}^{\infty} e^{-j \omega \tau} d \omega\right) d \tau \\
& =\int_{-\infty}^{\infty} R_{x x^{*}}(\tau) \delta(\tau) d \tau \\
& =R_{x x^{*}}(0) \\
& =E\left[x(t)^{2}\right]
\end{aligned}
$$

It is worth noting that noise signals are unilateral, thus by integrating over one half of the frequency spectrum the whole of the time averaged instantaneous power is known, as shown in Eq. (4.1.5).

$$
\overline{x(t)^{2}}=R_{x x^{*}}(0)=\lim _{T \rightarrow \infty} \frac{1}{T} \int_{0}^{T} x^{2}(t) d t
$$

\subsubsection{Intrinsic noise}

Intrinsic noise refers to the noise generated inside a device or circuit. The physical origin of noise in linear systems is due to the discrete nature of charge carriers. It is possible for the number of charge carriers in a specific plane to fluctuate with time. These fluctuations have a stochastic nature and are universal and unavoidable. The random behaviour of intrinsic noise leads to the adoption of statistical descriptions of noise characteristics [33].

Well-known examples of intrinsic noise sources are:

- Thermal noise: caused by the thermal agitation of charge carriers (electrons or holes) resulting in their random motion inside a piece of conductive material. Thermal noise is spectrally flat or has a uniform power spectral density and is independent of current flow [35]. Thermal noise is further discussed in section 4.2.

- Shot noise: described by Schottky as being due to the random passage of charge carriers across a barrier of potential, similar to a $p n$ junction. Schottky attributed the random fluctuations in current to the fact that current is composed not of a continuum but rather of a sequence of discrete increments of charge carriers with electrons arriving at random times. The average rate of charge arrival constitutes the direct current (DC) component while the superposition of a fluctuating component describe each discrete charge as it arrives [36]. Thus, shot noise is generally specified in terms of its mean-square variation about the average value as,

$$
\overline{i_{n}^{2}}=\int 2 q i_{D C} d f
$$


Where $q$ is the electron charge, $i_{D C}$ is the average value, and $d f$ is the differential frequency. The $q i_{D C}$ term is the current power spectral density in $\mathrm{A}^{2} / \mathrm{Hz}$.

Similar to thermal noise, shot noise is also spectrally flat but is independent of temperature. An example would be the shot noise generated from current impulses that are caused by the DC biasing current flowing through pn-junctions that are found in many semiconductor devices [36].

- Flicker noise: observed in circuits with DC signals, it displays a $1 / f$ characteristic. It is present in all active devices and has various origins. Its mean-square value is of the form,

$$
\overline{e_{n}^{2}}=\int \frac{K_{e}^{2}}{f} d f \quad \text { or } \quad \overline{i_{n}^{2}}=\int \frac{K_{i}^{2}}{f} d f .
$$

Where $K_{e}$ and $K_{i}$ are device specific constants in volts and amps respectively, $f$ is frequency, and $d f$ is differential frequency. The terms $K_{e}^{2} / f$ and $K_{i}^{2} / f$ are voltage and current power densities having units of $V^{2} / H z$ and $A^{2} / H z$.

Since flicker noise is a function of $1 / f$, it decreases substantially with an increase in frequency. Therefore, the DC signal should be taken into consideration when evaluating flicker noise [35].

\subsubsection{Extrinsic noise}

Extrinsic noise sources are situated outside the investigated circuit, the circuit acts as an antenna receiving the noise or spurious signals. For this reason extrinsic noise is also known as extraneous signals, spurious signals, or perturbations. According to its possible origins, two main categories of extrinsic noise exist [33]:

- Environmental perturbations are a broad noise classification including sky noise from strong broadband noise sources like the Sun, atmospheric noise caused mainly by lightning during thunderstorms, and man made noise such as electrical equipment, cellular telephones, radio and TV broad cast services [33].

- Crosstalk noise describes signals that are desirable in one circuit that pass via parasitic coupling into nearby circuits where they are undesirable, resulting in perturbations (or spurious signals). Coupling is usually a function of the relative proximity of various cables or equipment to one another.

\subsubsection{Noise character}

Since noise sources are stochastic they can be described by probability density functions, for example thermal noise and shot noise can be represented by Gaussian probability density functions. A Gaussian probability density function produces a uniform power spectral density that characterise a white noise source. By definition white noise has a zero mean,

$$
E[x(t)]=0, \quad \text { for all } t,
$$

with $E[x(t)]$ being the expected value of the random noise signal $x(t)$. White noise has unity variance,

$$
E\left[x(t)^{2}\right]=1, \quad \text { for all } t,
$$


and is time invariant,

$$
E[x(t) x(t-\tau)]=0, \quad \text { for } k \neq 0 \text { and all } t .
$$

Another example is Flicker noise, which is classified as pink noise because its power spectral density is inversely proportional to frequency,

$$
S(f) \propto \frac{1}{f} .
$$

\subsubsection{Intermediate-frequency receivers and noise}

In theory, intermediate frequency (IF) receivers have the potential to be more sensitive than an equivalent zero IF receiver because of the increased noise at lower frequencies caused by the flicker effect.

By selecting a high enough intermediate frequency, it is possible to render flicker noise insignificant compared to the Gaussian noise in the system. The reduction in noise results from the inverse frequency dependence of pink noise sources like flicker noise. This concept is shown to be true when concerned with the integration of operational amplifier noise (see section 4.6.1). According to [1], the improvement in receiver sensitivity with IF superheterodyne topologies, could in some cases be as high as $30 \mathrm{~dB}$ over the zero-IF receiver.

\subsection{Thermal noise}

Thermal noise is a major limiting factor to the useful sensitivity of any linear amplifier. A thermal noise source is described by a black body radiator operating in a single propagation mode. The first two laws of thermodynamics and Planck's relation provide an essential foundation to the theory of black body radiation [37]. The radiation is emitted according to Planck's law, meaning that its spectrum is determined by temperature alone, not by the body's shape or composition.

The thermodynamic laws of interest are:

- The first law of thermodynamics: in any closed system the total energy is constant, which is a restatement of the principle of the conservation of energy. Assuming that a closed system is one in which neither energy nor mass enter or leave [37].

- The second law of thermodynamics: in any closed system the entropy eventually maximizes, i.e. a closed system tends to distribute its energy randomly among the various degrees of freedom present in the system. Entropy is a measure of the number of specific ways in which a thermodynamic system can be arranged, thus it is a measure of randomness [37].

In 1927, John Bertrand Johnson, conducted a series of experiments that showed electrical noise exists in all conductors and that this noise is inherent and not the result of poor component design [38].

In 1928, Harry Nyquist, considered two conductors each of resistance $R$ and of the same uniform temperature $T$ connected in the manner indicated in Fig. 4.1. The electromotive force $\left(e_{n}\right)$ due to thermal agitation in conductor $I$ causes a current $\left(i_{n}\right)$ to be set up in 
the circuit. The value of the current is obtained by dividing the electromotive force by $2 R$ [39]. Equation (4.2.1) describes this deduction made by Nyquist.

$$
i_{n}=\frac{e_{n}}{2 R}
$$

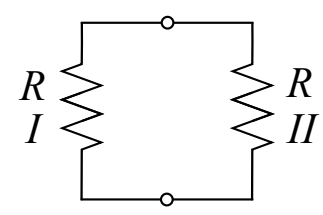

Figure 4.1: Nyquist's conductor setup for thermal noise description.

Planck's relation states, electromagnetic energy is radiated and absorbed in discrete quanta (photons) of energy. When referring to the quantisation of energy, it is implied that energy comes in 'discrete' quantities and not a continuous stream. Planck found that light of a certain frequency comes not as a continuous flow of energy, but it comes in little 'packets' called photons. The effect of quantization on the thermal energy in a single resonant mode is treated quantitatively using Planck's law, described by Eq. (4.2.3) [8]. According to Planck's relation, the energy $(E)$ of a photon is proportional to its frequency,

$$
E=h f
$$

where, $h$ is Planck's constant in Joule-seconds, and $f$ denotes frequency. Planck's law states that the average radiated energy of a single black body propagation mode in thermal equilibrium is given by,

$$
W(f)=\frac{h f}{e^{\frac{h f}{k T}}-1},
$$

where $k$ is Boltzmann's constant, $T$ is the temperature in Kelvin. It is possible to simplify Eq. (4.2.3) by applying the Rayleigh-Jeans approximation, producing Eq. (4.2.7). The approximation is achieved by taking the Maclaurin series expansion of Eq. (4.2.3)'s denominator,

$$
f\left(x=\frac{h f}{k T}\right)=e^{x}-1
$$

The Mclaurin series is given by Eq. (4.2.5), and represents is a special case of the more general Taylor series when expanded around the functions origin.

$$
f(x)=f(0)+f^{\prime}(0) x+\frac{f^{\prime \prime}(0)}{2 !} x^{2}+\ldots
$$

Equation (4.2.4) is approximated by truncating the Taylor polynomial's terms up to the first-order as in Eq. (4.2.6). The approximation is accurate for the average energy within a limited bandwidth $\Delta f$, while $h f / k T<<1$. Thus, the approximation is more accurate at higher temperatures and lower frequencies. It can be shown that at room temperature $(T=300 K)$ the approximation holds for frequencies in the Terahertz range.

$$
\begin{gathered}
f(x) \approx f(0)+f^{\prime}(0) x \\
W(f) \approx k T
\end{gathered}
$$


Nyquist showed using Johnson's experimental results, that for a matched load the average thermal noise power is equal to the average energy of a single black body propagation mode [38] [39],

$$
\hat{P}=W(f) d f \approx k T d f .
$$

The total power (into or out of the conductor) in a bandwidth of $\Delta f$ is described by Johnson's formula - Eq. (4.2.9), which gives the maximum available power from the noisy resistor at temperature $T$,

$$
\bar{P}=\int_{f_{0}}^{f_{0}+\Delta f} W(f) d f \approx \int_{f_{0}}^{f_{0}+\Delta f} k T d f=k T \Delta f,
$$

where $f_{0}$ is the bandwidth's starting frequency. From Eq. (4.2.1) and Eq. (4.2.9) it follows that, in terms of Planck's law, the mean square electromotive force of thermal noise generated by a conductor with resistance $R$ within a bandwidth $\Delta f$ is expressed as,

$$
\bar{P}=\overline{i_{n}^{2}} R=\left(\frac{\overline{e_{n}}}{2 R}\right)^{2} R=\frac{\overline{e_{n}^{2}}}{4 R} .
$$

Equating Eq. (4.2.9) and Eq. (4.2.10) results in the mean square thermal noise voltage,

$$
\overline{e_{n}^{2}}=4 k T R \Delta f \text {. }
$$

In the same way the mean square thermal noise current generated by a conductor $(G=$ $1 / R)$ can be expressed as follows,

$$
\overline{i_{n}^{2}}=4 k T G \Delta f
$$

Equations (4.2.11) and (4.2.12) can be used to represent any noisy resistor with a noiseless Thévenin or Norton equivalent circuit model as illustrated in Fig. 4.2. The equivalent circuit models are useful when analysing noise in linear circuits.

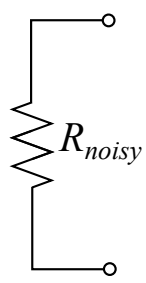

(a)

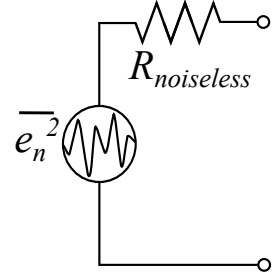

(b)

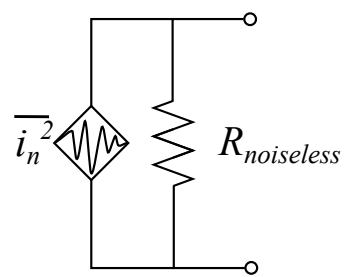

(c)

Figure 4.2: (a) Noisy resistor, (b) Thévenin equivalent circuits, (c) Nortan equivalent circuit.

Noise power, as defined by Eq. (4.2.9), is independent of frequency, i.e. the noise source's power spectral density is constant with varying frequency and is a white noise source. Independent white noise sources can be treated as Gaussian-distributed random variables, thus the noise power of independent noise sources are additive [8].

On closer inspection of Eq. (4.2.9) the following is observed:

- $\Delta f \rightarrow 0, P_{n} \rightarrow 0$. As the receiver's bandwidth tends to zero less noise power is collected.

- $T \rightarrow 0, P_{n} \rightarrow 0$. Decreasing the device temperature results in the component generating less noise power. 


\subsubsection{Equivalent noise temperature}

Consider an arbitrary white noise source (thermal or non-thermal) as depicted in Fig. 4.3. White noise signals have a uniform power spectral density, thus the noise power is not a function of frequency over the desired bandwidth. The noise source is modelled as an equivalent thermal noise source that is characterized by an equivalent noise temperature.

The arbitrary white noise source in Fig. 4.3 has a characteristic impedance of $R$ and delivers a noise power $N_{0}$ to a matched load resistor, $R$. The noise source can be replace by a noisy resistor of value $R$ at an equivalent noise temperature of $T_{e}$. The equivalent noise temperature is selected so that the same noise power is delivered to the load [8], i.e.

$$
T_{e}=\frac{N_{0}}{k \Delta f}
$$

Eq. (4.2.13) is restated so that the noise power is in terms of the noise source's equivalent noise temperature,

$$
N_{0}=k T_{e} \Delta f
$$

This result allows components and systems to be characterized by an equivalent noise temperature $T_{e}$. It is clear from Eq. (4.2.13) that the network's bandwidth is assumed to be fixed.

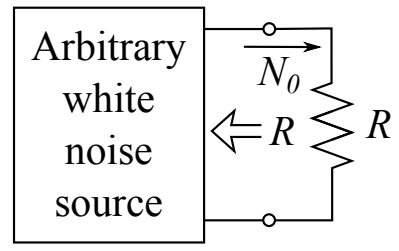

(a)

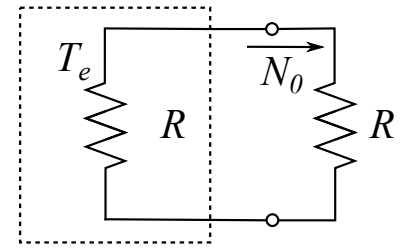

(b)

Figure 4.3: (a) An arbitrary white noise source generating a noise power of $N_{0}$. (b) An equivalent noise temperature $\left(T_{e}\right)$ and matched impedance $(R)$ to characterise the arbitrary white noise source.

\subsection{Noise figure}

Noise factor $(\mathrm{F})$ is a measure of the reduction in signal-to-noise ratio (SNR),

$$
F=\frac{S_{i} / N_{i}}{S_{o} / N_{o}}=\frac{1}{G} \frac{N_{o}}{N_{i}} \geq 1
$$

where $S_{i}$ and $S_{o}$ are the input and output signals, while $N_{i}$ and $N_{o}$ are the input and output noise. The signal-to-noise ratio is the ratio of desired signal power to undesired noise power [8]. As described in section 4.2, the input noise power is due to a matched resistor at a uniform environmental temperature of $T_{0}$,

$$
N_{i}=k T_{0} \Delta f
$$

Consider a noisy two-port network, as shown in Fig. 4.4, that has an input noise and signal power of $N_{i}$ and $S_{i}$ respectively. The network is characterized by an equivalent noise temperature $T_{e}$, gain $G$, and bandwidth $\Delta f$. Given an input noise power as described 
by Eq. (4.3.2), the output noise power is the sum of the amplified input noise and the internally generated noise,

$$
N_{o}=k G \Delta f\left(T_{0}+T_{e}\right) .
$$

The output signal power is equal to the amplified input power,

$$
S_{o}=G S_{i}
$$

Applying these results to Eq. (4.3.1) gives the noise factor of the two-port network,

$$
F=\frac{S_{i}}{k T_{0} \Delta f} \frac{k G \Delta f\left(T_{0}+T_{e}\right)}{G S_{i}}=1+\frac{T_{e}}{T_{0}} .
$$

Solving for the equivalent noise temperature $T_{e}$ of the network results in,

$$
T_{e}=(F-1) T_{0} .
$$

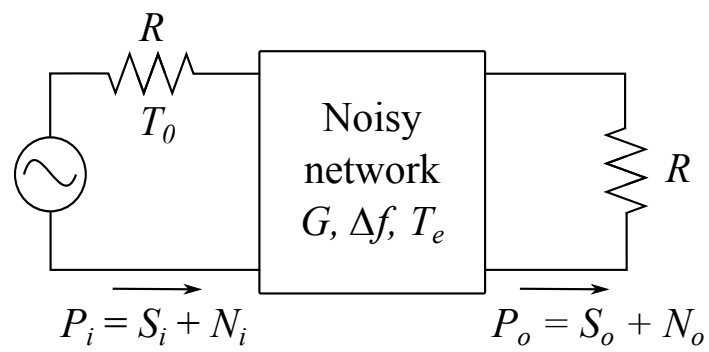

Figure 4.4: Noisy two-port network.

Noise factor $(\mathrm{F})$ is a linear unit that can be converted to decibel $(\mathrm{dB})$. The $\mathrm{dB}$ scaled noise factor is referred to as noise figure (NF),

$$
N F=10 \log (F) \geq 0 \text {. }
$$

From Eq. (4.3.6) it is seen that noise figure and equivalent noise temperature $\left(T_{e}\right)$ are interchangeable characterizations of the noise properties of a network. Therefore, a noisy network's output noise power is given in terms of its noise factor by substituting Eq. (4.3.6) into Eq. (4.3.3), resulting in Eq. (4.3.8).

$$
N_{o}=G N_{i}+k G \Delta f(F-1) T_{0}
$$

\subsubsection{Noise figure of a cascaded system}

RF receivers and microwave systems usually consist of many different cascaded components. Each stage in the receiver chain degrades the SNR by some degree. The cascaded noise figure $\left(N F_{\text {cas }}\right)$ is an indicator of the degradation in the receiver's SNR. By knowing the NF of the individual stages in a receiver chain, it is possible to determine the cascaded noise figure of the receiver.

Figure 4.5 depicts the general case where a finite number of stages are cascaded $(n \geq$ 1). Using the first component's equivalent noise temperature, the noise power at the output of the first stage can be calculated as,

$$
N_{1}=G_{1}\left(N_{i}+k \Delta f T_{e 1}\right)=G_{1} k T_{0} \Delta f+G_{1} k T_{e 1} \Delta f .
$$




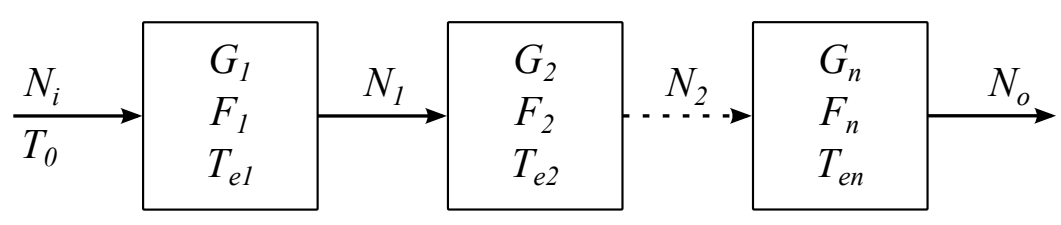

Figure 4.5: General $n$ stage cascaded network, where each stage is represented by its gain, noise figure, and equivalent noise temperature.

By generalising Eq. (4.3.9) the noise power at the output of the $n$-th stage is determined

$$
\begin{aligned}
N_{o} & =G_{n}\left(N_{n-1}+k \Delta f T_{e n}\right), \\
& =\left(G_{1} G_{2} \cdots G_{n}\right) k \Delta f\left(T_{0}+T_{e 1}+\frac{1}{G_{1}} T_{e 2}+\cdots+\frac{1}{G_{1} G_{2} \cdots G_{n}} T_{e n}\right), \\
& =\prod_{i=1}^{n} G_{i} k \Delta f\left(T_{0}+\sum_{q=1}^{n} \frac{T_{e q}}{\prod_{p=0}^{q-1} G_{p}}\right), \quad G_{0}=1, n \geq 1 .
\end{aligned}
$$

The total cascaded noise factor, $F_{\text {cas }}$, is derived from Eq. (4.3.10) by applying Eq. (4.3.1) and Eq. (4.3.6),

$$
\begin{aligned}
F_{c a s} & =\frac{S_{i}}{N_{i}} \frac{N_{o}}{S_{o}} \\
& =\frac{S_{i}}{k \Delta f T_{0}} \frac{\left(G_{1} G_{2} \cdots G_{n}\right) k \Delta f\left(T_{0}+T_{e 1}+\frac{1}{G_{1}} T_{e 2}+\cdots+\frac{1}{G_{1} G_{2} \cdots G_{n}} T_{e n}\right)}{\left(G_{1} G_{2} \cdots G_{n}\right) S_{i}}, \\
& =F_{1}+\frac{F_{2}-1}{G_{1}}+\frac{F_{3}-1}{G_{1} G_{2}}+\cdots, \\
& =1+\sum_{i=1}^{n} \frac{F_{i}-1}{\prod_{q=0}^{i-1} G_{q}}, \quad G_{0}=1, n \geq 1 .
\end{aligned}
$$

From Eq. (4.3.11) it is clear that the first few stages in a receiver chain dominates the noise factor of a cascaded network. For each additional stage added to the cascaded network the noise factor is divided by the preceding stages compound gain. For this reason many receivers have a low noise amplifier (LNA) as its first stage, due to the LNA's high gain and low noise figure.

\subsubsection{Noise figure of passive two-port networks}

When determining the noise figure of two port networks, a special case may occur where the network consists of lossy passive components, for example an attenuator or lossy transmission line held at a physical temperature $T$. The power gain $G$ of a lossy passive network is less than unity, hence a loss factor $L$ can be defined as $L=1 / G<1$ [8].

The noise figure of a matched lossy two-port network can be determined by using a thermodynamic argument. Assuming that the input noise power to a passive network is $N_{i}=k T \Delta f$, and that the network is in thermodynamic equilibrium the available output noise power is calculated as,

$$
N_{o}=k T \Delta f=G k T \Delta f+G N_{\text {added }} .
$$

Equation (4.3.12) is used to solve for the noise added by the matched passive network

$$
N_{\text {added }}=\left(\frac{1-G}{G}\right) k T \Delta f=(L-1) k T \Delta f .
$$


Given that $N_{\text {added }}=k T_{e} \Delta f$ is equal to Eq. (4.3.13), the equivalent noise temperature added by a lossy network is given by Eq. (4.3.14) [8].

$$
T_{e}=\left(\frac{1-G}{G}\right) T=(L-1) T .
$$

By applying Eq. (4.3.1) the noise figure is determined

$$
F=1+\left(\frac{1-G}{G}\right) \frac{T}{T_{0}}=1+(L-1) \frac{T}{T_{0}} .
$$

For the specific case where the passive network's temperature $T$ is equal to the uniform environmental temperature $T_{0}$, the noise figure is equal to the loss factor of the network

$$
F=L
$$

Further generalization of Eq. (4.3.15) is needed to account for the change in noise figure due to impedance mismatch at either the input or output port of the passive network. In the case of active devices it is generally easier and more accurate to measure the noise characteristics rather than calculating it from first principles.

\subsubsection{Noise figure as a figure of merit}

Noise figure is a useful, yet restricted figure of merit. The object of a good receiver design is to establish the best SNR. With all other factors being equal (dynamic range, bandwidth, transient response, demodulation techniques, etc.) the receiver with the lower noise figure is better. Therefore it is important to measure and calculate the noise figure (or the noise temperature) of a system accurately [40].

For the purposes of this thesis, noise figure and noise temperature are defined in basic terms. Noise figure is a measure of the degradation of the SNR available at the input terminals as a signal passes through the linear portions of the receiver. Since the temperature at the input termination is fixed and specified, the noise figure is essentially independent of the antenna characteristics. Thus, the difference between the noise figures of two receivers is not a direct measure of the change in performance when comparing actualised systems. Receiver parameters and characteristics, like selectivity, bandwidth, frequency response, receiver topology etc., have an impact on the resultant output SNR.

\subsection{Adding noise sources}

To determine a network's cascaded output noise signal, multiple independent noise sources are combined. The calculations are simplified by only taking uncorrelated noise into account. Correlated noise contribute to a small fraction of the total noise generated by a circuit. In the case of an amplifier's internal noise sources, less than 10 to 15 percent of the total noise is correlated [41].

To illustrate the concept, two resistors, $R_{1}$ and $R_{2}$ with their Thévenin equivalent noise generators $\overline{e_{1}^{2}}$ and $\overline{e_{2}^{2}}$, are connected in series as shown in Fig. 4.6. The mean-square voltage $\overline{e_{t}^{2}}$ across the resistors, where $e_{t}(t)=e_{1}(t)+e_{2}(t)$ is the instantaneous noise value, is given by

$$
\overline{e_{t}(t)^{2}}=\overline{\left[e_{1}(t)+e_{2}(t)\right]^{2}}=\overline{e_{1}(t)^{2}}+\overline{e_{2}(t)^{2}}+\overline{2 e_{1}(t) e_{2}(t)} .
$$


The noise voltages $e_{1}(t)$ and $e_{2}(t)$ are white noise sources and they are independently generated by two different resistors. The average of the product of the noise voltages is zero, $\overline{2 e_{1}(t) e_{2}(t)}=0$, resulting in

$$
\overline{e_{t}^{2}}=\overline{e_{1}^{2}}+\overline{e_{2}^{2}}
$$

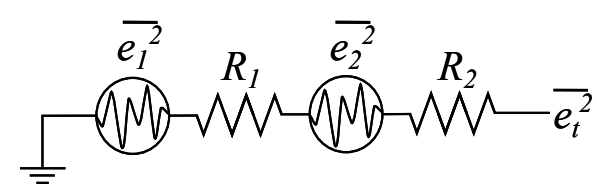

Figure 4.6: Series noise sources, $\overline{e_{1}^{2}}$, and $\overline{e_{2}^{2}}$, generated by resistors, $R_{1}$, and $R_{2}$.

In general Eq. (4.4.2) holds for more than two noise sources, given that the sources arise from separate mechanics and are statistically independent. The mean-square value of a sum of statistically independent noise sources is the sum of the individual mean-square values [35].

\subsection{Equivalent noise bandwidth}

The spectrum of any input noise signal presented to a system, with a transfer function $H(s)$, is shaped according to its frequency response. For white noise sources with constant power over frequency, it is convenient to replace the transfer function $H(s)$ by a "brickwall" filter (or step function) as depicted in Fig. 4.7.

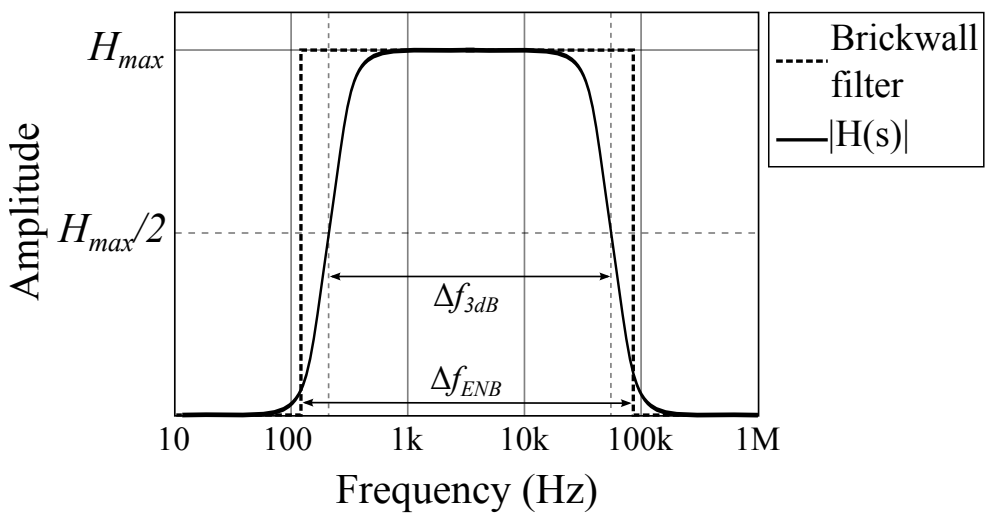

Figure 4.7: Equivalent noise bandwidth (ENB) "brickwall" representation of a system's transfer function $H(s)$.

A network's frequency response can be redefined as a "brickwall", where its total output noise power is equal to the network's transfer function $H(s)$. This bandwidth is known as the equivalent noise bandwidth (ENB) and the area under the ENB is equal to the area under the network's transfer function $H(s)$ [42],

$$
\int_{0}^{\infty}|H(j \omega)|^{2} d \omega=\underbrace{2 \pi H_{\text {max }}^{2} \Delta f_{E N B}}_{\text {Area under rectangular function }},
$$

where $H_{\max }$ is the maximum value of $H(s)$. The ENB is given by

$$
\Delta f_{E N B}=\frac{1}{2 \pi} \int_{0}^{\infty} \frac{|H(j \omega)|^{2}}{H_{\max }^{2}} d \omega
$$


A system characterised by a first order low-pass filter has the following transfer function

$$
\begin{aligned}
H(j \omega) & =\frac{H_{\max }}{1+j \frac{\omega}{\omega_{3 d B}}}, \\
|H(j \omega)| & =\frac{H_{\max }}{\sqrt{1+\left(\frac{\omega}{\omega_{3 d B}}\right)}},
\end{aligned}
$$

and its ENB is calculated using Eq. (4.5.2),

$$
\begin{aligned}
f_{E N B} & =\frac{1}{2 \pi} \int_{0}^{\infty} \frac{1}{1+\left(\frac{\omega}{\omega_{3 d B}}\right)^{2}} d \omega \\
& =\left.\frac{\omega_{3 d B}}{2 \pi} \tan ^{-1}\left(\frac{\omega}{\omega_{3 d B}}\right)\right|_{0} ^{\infty}, \\
& =f_{3 d B} \frac{\pi}{2}, \\
& =f_{3 d B} C_{E N B},
\end{aligned}
$$

where $C_{E N B}$ is the ENB scaling coefficient.

Table 4.1: Equivalent noise bandwidth (ENB) coefficients for low-pass filter responses

\begin{tabular}{c|c}
\hline \hline \multicolumn{2}{c}{ Low-pass } \\
filter response \\
\hline Order & $C_{E N B}$ \\
\hline 1 & 1.57 \\
2 & 1.22 \\
3 & 1.15 \\
4 & 1.13 \\
5 & 1.11 \\
\hline
\end{tabular}

Tabulated values of ENB coefficients for higher order low-pass filter responses are provided in Table 4.1 from [35]. Networks that exhibit higher order behaviour (sharper role-off) are closer to the idealised rectangular filter response, thus the ENB scaling coefficient are closer to unity.

\subsection{Amplifier noise}

In general, a noisy two-port network can be simplified to a model consisting of a noiseless network with external noise sources [43]. A simple noise model used to represent noisy two-port amplifiers consists of two noise sources connected to a noiseless amplifier's input as in Fig. 4.8. The amplifier's noise sources $e_{n}$ and $i_{n}$ are not necessarily uncorrelated even though in most cases this is assumed.

A Thévenin equivalent source is applied to the amplifier's input, where $v_{s}$ is the source voltage, $Z_{s}=R_{s}+j X_{s}$ is the source impedance, and $V_{t s}$ is the thermal noise voltage generated by the source [44]. All the impedances in the analysis are assumed to be time invariant. Summing the input noise sources produces an equivalent noise voltage

$$
v_{n i}=v_{t s}+e_{n}+i_{n} Z_{s} .
$$


Equation (4.6.1) is independent of both the gain $(A)$ and input impedance $\left(Z_{i}\right)$ and describes the noise voltage across the input to the noiseless amplifier when $Z_{i}$ is considered to be an open circuit.

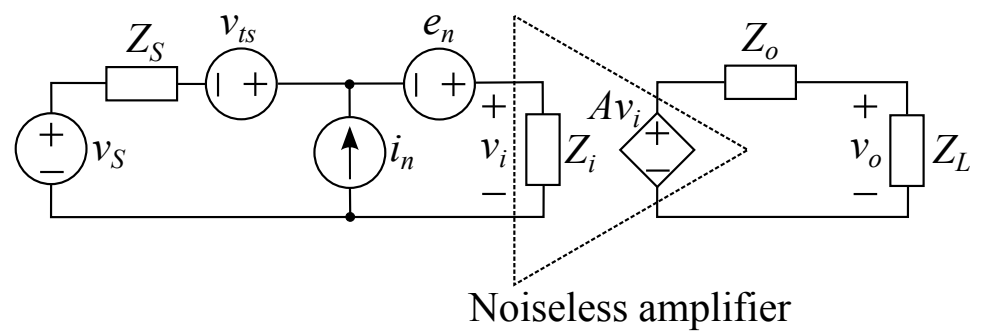

Figure 4.8: $e_{n}-i_{n}$ amplifier noise model represented by instantaneous noise sources, a Thévenin equivalent input source is applied to the network.

The mean-square value of $v_{n i}$ is calculated and it is assumed that all except the internally generated noise sources are uncorrelated,

$$
\begin{aligned}
\overline{v_{n i}^{2}} & =\overline{\left(v_{t s}+e_{n}+i_{n} Z_{s}\right)\left(v_{t s}+e_{n}+i_{n} Z_{s}\right)^{*}}, \\
& =\overline{v_{t s} v_{t s}^{*}}+\overline{e_{n} e_{n}^{*}}+2 \operatorname{Re}\left\{\overline{e_{n} i_{n}^{*}} Z_{s}\right\}+\overline{\left(i_{n} Z_{s}\right)\left(i_{n}^{*} Z_{s}^{*}\right)}, \\
& =4 k T \operatorname{Re}\left\{Z_{s}\right\} \Delta f+\overline{e_{n}^{2}}+2 \operatorname{Re}\left\{\overline{e_{n} i_{n}^{*}} Z_{s}\right\}+\overline{i_{n}^{2}}\left|Z_{s}\right|^{2} .
\end{aligned}
$$

Calculate the output noise voltage as

$$
\begin{aligned}
v_{n o} & =A \frac{Z_{L}}{Z_{L}+Z_{o}} v_{i}, \\
& =A \frac{Z_{L}}{Z_{L}+Z_{o}} \frac{Z_{i}}{Z_{i}+Z_{S}}\left(v_{t s}+e_{n}+i_{n} Z_{s}\right), \\
& =A \frac{Z_{L}}{Z_{L}+Z_{o}} \frac{Z_{i}}{Z_{i}+Z_{S}} v_{n i} .
\end{aligned}
$$

The output voltage due to the input source is

$$
v_{o}=A \frac{Z_{L}}{Z_{L}+Z_{o}} \frac{Z_{i}}{Z_{i}+Z_{S}} v_{s}
$$

The noise factor of the amplifier in Fig. 4.8 is determined as follows

$$
\begin{aligned}
F & =\frac{\overline{v_{s}^{2}} / \overline{v_{t s}^{2}}}{\overline{v_{o}^{2}} / \overline{v_{n o}^{2}}} \\
& =\frac{\overline{v_{n i}^{2}}}{\overline{v_{t s}^{2}}}, \\
& =1+\frac{\overline{e_{n}^{2}}+2 \operatorname{Re}\left\{\overline{e_{n} i_{n}^{*}} Z_{s}\right\}+\overline{i_{n}^{2}}\left|Z_{s}\right|^{2}}{\overline{v_{t s}^{2}}} .
\end{aligned}
$$

When doing noise calculations for impedance mismatched amplifiers, it is useful to represent noise sources as voltage and current spectral densities. In section 4.7 this approach is followed in the noise analyses of operational amplifier (op-amp) circuits [45]. 


\subsubsection{Integrating operational amplifier noise}

An op-amp's intrinsic noise sources contains both pink noise (or $1 / f$ noise) and white noise. The effect of both noise types are integrated and the total mean-square noise is calculated. In the following analysis the voltage representation of noise is used but the same result would hold for the equivalent current representation.

Given a white noise source with a voltage spectral density of $S_{w}(f)=e_{n}^{2}$. The white noise mean-square voltage is determined as follows

$$
\overline{e_{w}^{2}}=\int_{f_{L}}^{f_{H}} e_{n}^{2} d f=e_{n}^{2}\left(f_{H}-f_{L}\right)
$$

where $e_{n}$ is the square of the white noise voltage specification for the op-amp in $\mathrm{V} / \sqrt{\mathrm{Hz}}$, also $f_{L}$ and $f_{H}$ are the lowest and highest frequencies of interest. Next a pink noise source is defined

$$
\overline{e_{p}^{2}}=\int_{f_{L}}^{f_{H}} \frac{K_{e}^{2}}{f} d f=K_{e}^{2} \ln \left(\frac{f_{H}}{f_{L}}\right),
$$

where $\overline{e_{p}^{2}}$ is the pink noise mean-square voltage, and $K_{e}$ is the device constant in volts.

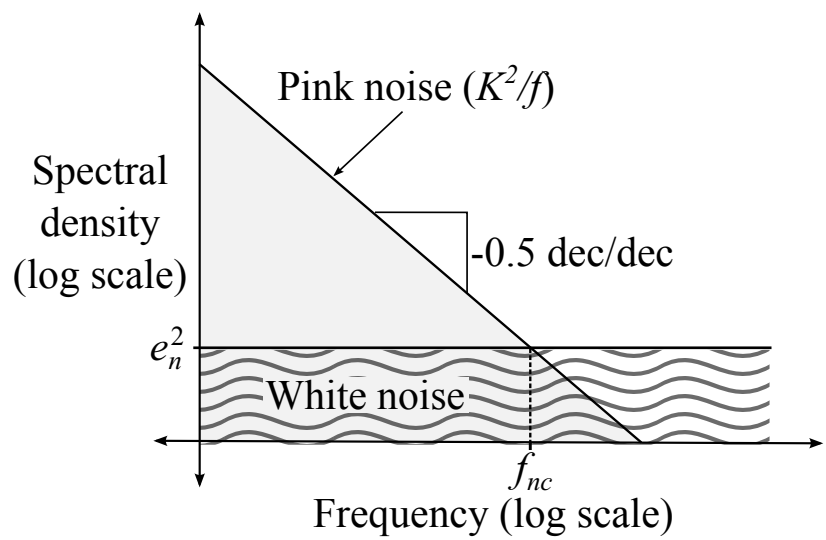

Figure 4.9: Pink and white noise intersect point at $f_{n c}$ on a log-log scale.

When plotting the total effective input noise of the op-amp against frequency on a $\log -\log$ scale, $1 / f$ noise is a straight line that has a constant slope. If the voltage spectral density is graphed, the slope is equal to $-0.5 \mathrm{dec} / \mathrm{dec}$. The point in the frequency spectrum where the noise contributions by pink and white noise are equal is referred to as the noise corner frequency, $f_{n c}$, depicted in Fig. 4.9 [35]. Thus the noise corner frequency is

$$
\begin{array}{r}
e_{n}^{2}=\frac{K_{e}^{2}}{f_{\text {enc }}}, \\
f_{\text {enc }}=\frac{K_{e}^{2}}{e_{n}^{2}},
\end{array}
$$

where $f_{\text {enc }}$ is the voltage noise corner frequency, and $K_{e}$ is the device specific constant in volts. The total mean-square noise (in $V^{2}$ ) is calculated by adding Eq. (4.6.6) and Eq. $(4.6 .7)$,

$$
\overline{e_{t}^{2}}=e_{n}^{2}\left[f_{\text {enc }} \ln \left(\frac{f_{H}}{f_{L}}\right)+\left(f_{H}-f_{L}\right)\right] .
$$


The voltage noise corner frequency $\left(f_{\text {enc }}\right)$ of the LT6200 op-amp by Linear Technologies is determined visually from a graph depicting the equivalent input noise voltage versus frequency as illustrated by Fig. 4.10 (a). The current noise corner frequency $\left(f_{\text {inc }}\right)$ is determined from the equivalent balanced/unbalanced noise current versus frequency graphs shown in Fig. 4.10 (b) and (c) respectively.

\section{Input Noise Voltage vs Frequency}

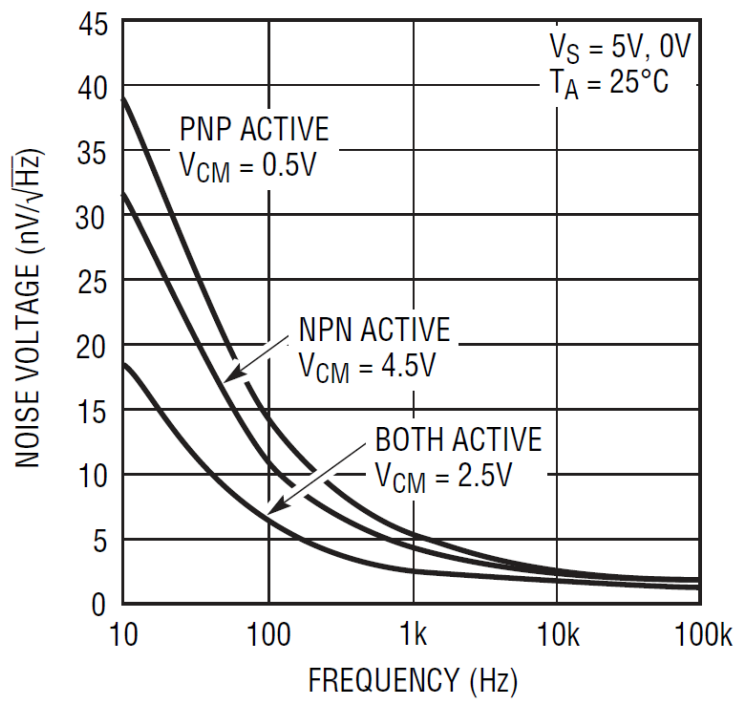

(a)

Balanced Noise Current vs Frequency

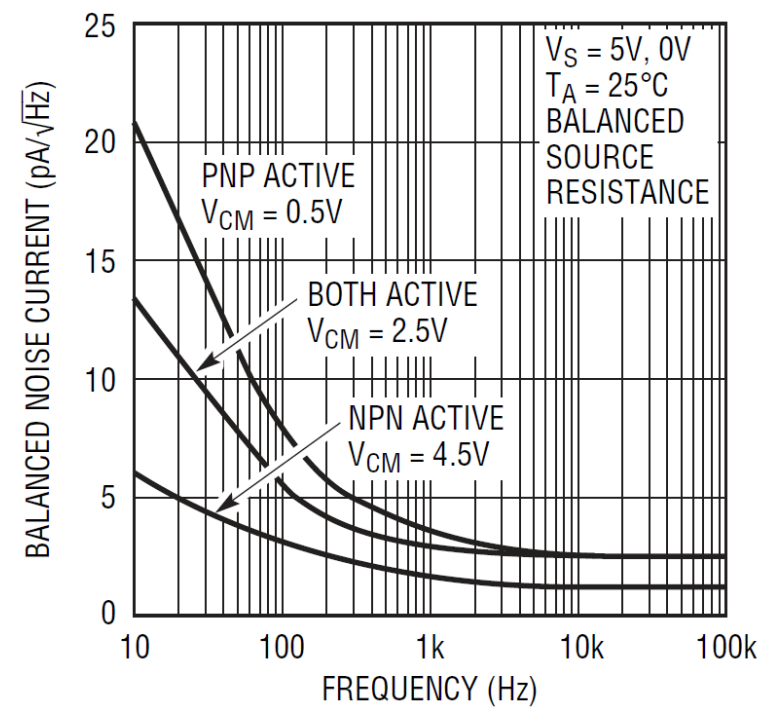

(b)
Unbalanced Noise Current vs Frequency

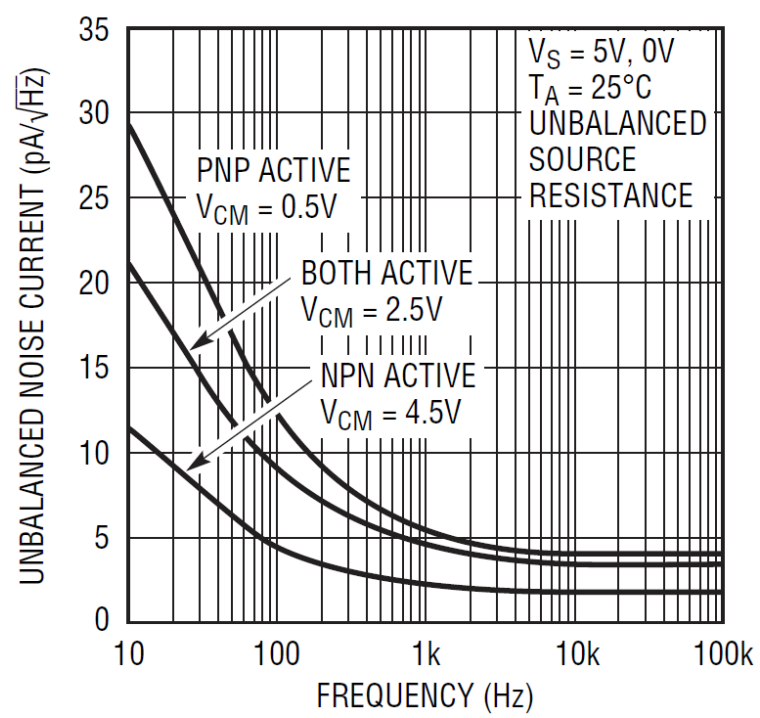

(c)

Figure 4.10: Linear Technology's LT6200 op-amp's input noise versus frequency plots [46].

To determine the voltage and current noise corner frequencies, the device specific constant $K$ is required in volts $\left(K_{e}\right)$ and amperes $\left(K_{i}\right)$. The value of $K^{2}$ is determined by examining the input noise versus frequency graph. The equivalent input noise $\left(e_{n i}\right)$ at the lowest possible frequency $\left(f_{\text {low }}\right)$ in the $1 / f$ noise region is determined from Fig. 4.10. The white noise voltage squared is subtracted from $e_{n i}^{2}$ and the result is multiplied by the 
known frequency $f_{\text {low }}$ as in Eq. (4.6.10).

$$
\begin{aligned}
K_{e}^{2} & =\left(e_{n i}^{2}-e_{n}^{2}\right) f_{\text {low }} \\
& =\left[(18 n V / \sqrt{H z})^{2}-(1.1 n V / \sqrt{H z})^{2}\right] \times(10 H z) \\
& =3227.9(n V)^{2}
\end{aligned}
$$

The noise corner frequency $f_{n c}$ is calculated by applying Eq. (4.6.8), where $K^{2}$ is divided by the op-amp's white noise specification squared $\left(e_{w}^{2}\right)$, as shown in Eq. (4.6.11) [35].

$$
\begin{aligned}
f_{\text {enc }} & =\frac{K_{e}^{2}}{e_{n}^{2}} \\
& =\frac{3227.9(n V)^{2}}{(1.1 n V / \sqrt{H z})^{2}} \\
& =2.668 \mathrm{kHz}
\end{aligned}
$$

Thus, at $2.67 \mathrm{kHz}$ the LT6200 op-amp's pink and white noise contributions are theoretically equal. This is a low frequency considering that the LT6200 op-amp has a unity gain-bandwidth of $70 \mathrm{MHz}$. Therefore, the LT6200's pink noise contribution would be negligible if used at a centre frequency of $1.05 \mathrm{MHz}$ in a receiver with a $100 \mathrm{kHz}$ bandwidth. Section 4.6.2 utilises the result to calculate an op-amp's overall rms voltage noise.

\subsubsection{Operational amplifier noise}

Analysing the noise of op-amp circuits are done in much the same way as for the generic two-port amplifier of section 4.6. However, in the case of an op-amp the noise figure is not only dependant on the source impedance and device characteristics, it is also dependent on a feedback network [35].

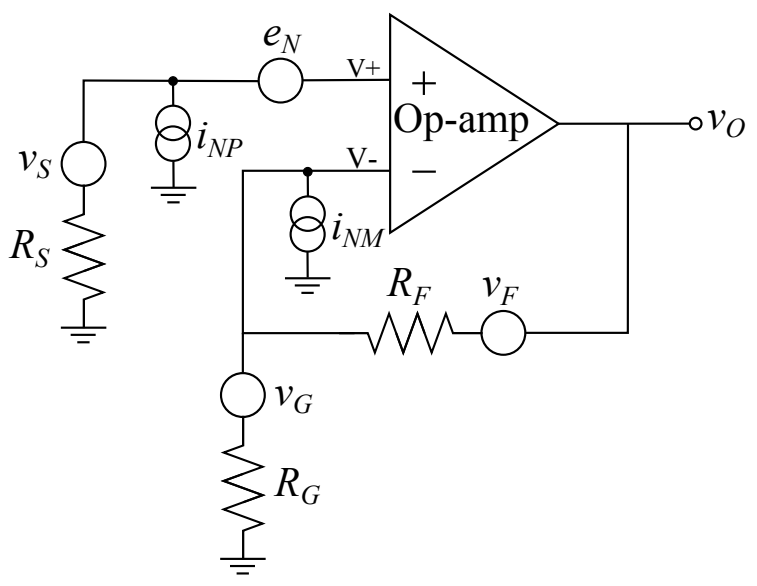

Figure 4.11: Non-inverting operational amplifier noise model.

The op-amp noise calculations are simplified by assuming that the op-amp exhibits ideal behaviour. The characteristics of an ideal op-amp are, infinite voltage gain, infinite input impedance, zero output impedance, infinite bandwidth, and zero offset voltage [47].

The op-amp noise specification presented on a data sheet usually refers the measured noise to the input of the op-amp. The input referred voltage noise $\left(e_{N}\right)$ is in units of $V / \sqrt{H z}$, and the input current noise $\left(i_{N M}\right.$ and $\left.i_{N M}\right)$ is in $A / \sqrt{H z}$ [48]. When performing 
an op-amp noise analysis, the input referred noise models are added to the circuit. Nonnoise sources are terminated in either a short or an open circuit, where voltage sources are shorted and current sources opened [35]. This procedure produces similar noise models for an inverting and a non-inverting op-amp circuit.

The non-inverting op-amp noise model is shown in Fig. 4.11, where the voltage noise gain seen from the non-inverting port is given by

$$
G_{n p}=\frac{R_{G}+R_{F}}{R_{G}}=1+\frac{R_{F}}{R_{G}} .
$$

For the noise calculations done in this section, only the uncorrelated noise sources are considered, since correlated noise contributes to a low percentage of the total noise [41].

In Fig. 4.12 all the voltage noise sources are referred to the input of a noiseless amplifier. These sources can be moved from the input to the output by multiplying by the voltage gain [35].

The rms noiseless input signal and rms noise generated by the input's equivalent resistance $R_{S}$ are represented by $\overline{v_{i}}$ and $\overline{v_{s}}$ respectively. The other noise sources depicted in Fig. 4.12 are either generated by the resistive elements in the circuit or the op-amp's own internal noise generators as illustrated in Fig. 4.11.

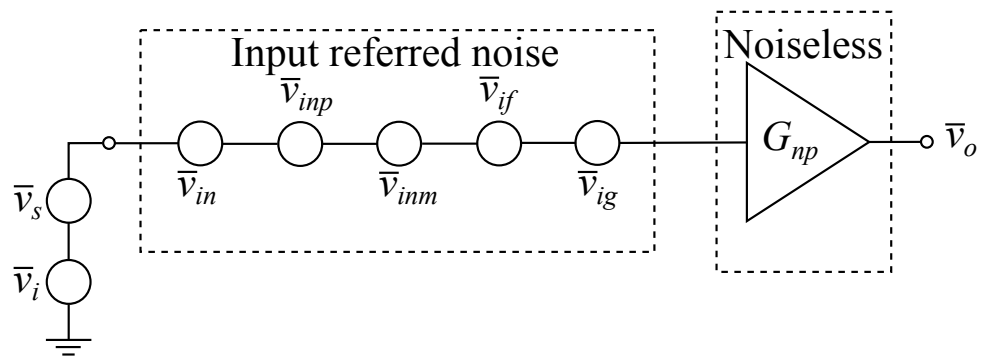

Figure 4.12: The root-mean-square input referred noise to the non-inverting operational amplifier.

The superposition principle is used to analyse the multi-source circuit and applies as long as the system remains linear. It states that the net response at a given node is the sum of the responses caused by each stimulus individually. The superposition principle is described by combining the homogeneity and additivity properties given by Eq. (4.6.13) and Eq. (4.6.14) respectively [47].

$$
\begin{gathered}
F(a x)=a F(x) \\
F\left(x_{1}+x_{2}+\cdots\right)=F\left(x_{1}\right)+F\left(x_{2}\right)+\cdots .
\end{gathered}
$$

The noise analysis is conducted in two parts; first the noise due to the resistors are considered and then the internal noise generated by the op-amp is added.

The noise generated by resistors $R_{S}, R_{G}$, and $R_{F}$ are modelled as thermal voltage noise sources [8]. Each source's mean-square noise voltage is calculated and referred to the op-amp's output ( $v_{o}$ of Figure 4.11$)$. The relevant equations are presented in Table 4.2 .

From Table 4.2 the total output rms voltage noise $\left(\overline{v_{o r}}\right)$ due to the resistors is calculated,

$$
\begin{aligned}
\bar{v}_{o r} & =\sqrt{\bar{v}_{o s}^{2}+\bar{v}_{o g}^{2}+\bar{v}_{o f}^{2}}, \\
& =\sqrt{\int\left[4 k T R_{S}\left(\frac{R_{G}+R_{F}}{R_{G}}\right)^{2}+4 k T R_{F}\left(\frac{R_{G}+R_{F}}{R_{G}}\right)\right] d f} .
\end{aligned}
$$


Table 4.2: Non-inverting op-amp circuit's feedback network thermal voltage noise

\begin{tabular}{c|c|c}
\hline \hline $\begin{array}{c}\text { Resistive } \\
\text { elements }\end{array}$ & $\begin{array}{c}\text { Source mean-square } \\
\text { voltage noise }\end{array}$ & $\begin{array}{c}\text { Output mean-square } \\
\text { voltage noise }\end{array}$ \\
\hline$R_{S}$ & $\bar{v}_{s}^{2}=\int 4 k T R_{S} d f$ & $\bar{v}_{o s}^{2}=\bar{v}_{s}^{2} G_{n p}^{2}$ \\
$R_{G}$ & $\bar{v}_{g}^{2}=\int 4 k T R_{G} d f$ & $\bar{v}_{o g}^{2}=\bar{v}_{g}^{2}\left(\frac{R_{F}}{R_{G}}\right)^{2}$ \\
$R_{F}$ & $\bar{v}_{f}^{2}=\int 4 k T R_{F} d f$ & $\bar{v}_{o f}^{2}=\bar{v}_{f}^{2}$ \\
\hline
\end{tabular}

The resistive network's noise $\left(\overline{v_{o r}}\right)$ is referred to the input $\left(\overline{v_{i r}}\right)$ by dividing Eq. (4.6.15) by the noise gain $\left(G_{n p}\right)$,

$$
\begin{aligned}
\bar{v}_{i r}^{2} & =\left(\frac{\bar{v}_{\text {or }}}{G_{n p}}\right)^{2}, \\
& =\int 4 k T\left(R_{F} \| R_{G}+R_{S}\right) d f .
\end{aligned}
$$

The parallel combination of $R_{G}$ and $R_{F}$ is set equal to the source impedance $\left(R_{S}\right)$ to minimize the offset voltage due to the input bias current [35]. This reduces Eq. (4.6.16) to

$$
\bar{v}_{i r}^{2}=\int 8 k T R_{S} d f .
$$

Next the internal noise sources associated with the op-amp are considered. The analysis proceeds as before by continuing to apply superposition and referring the noise to the op-amp's output (see Table 4.3).

Table 4.3: Output referred op-amp internal noise sources

\begin{tabular}{c|c|c}
\hline \hline $\begin{array}{c}\text { Internal noise } \\
\text { source }\end{array}$ & $\begin{array}{c}\text { Source mean-square } \\
\text { voltage noise }\end{array}$ & $\begin{array}{c}\text { Output mean-square } \\
\text { voltage noise }\end{array}$ \\
\hline$e_{n}$ & $\bar{v}_{n}^{2}=\int e_{n}^{2} d f$ & $\bar{v}_{o n}^{2}=\bar{v}_{n}^{2} G_{n p}^{2}$ \\
$i_{n p}$ & $\bar{v}_{n p}^{2}=\int\left(i_{n p} R_{S}\right)^{2} d f$ & $\bar{v}_{o n p^{2}}=\bar{v}_{n p}^{2} G_{n p}^{2}$ \\
$i_{n m}$ & $\bar{v}_{n m}^{2}=\int\left(i_{n m} R_{F}\right)^{2} d f$ & $\bar{v}_{o n m}^{2}=\bar{v}_{n m}^{2}$ \\
\hline
\end{tabular}

The resistive component and op-amp internal noise sources are combined as a sum of squares, resulting in the total output rms voltage noise generated by the op-amp circuit [35],

$$
\begin{aligned}
\bar{v}_{o} & =\sqrt{\bar{v}_{o s}^{2}+\bar{v}_{o g}^{2}+\bar{v}_{o f}^{2}+\bar{v}_{o n}^{2}+\bar{v}_{o n p}^{2}+\bar{v}_{o n m}^{2}}, \\
& =\sqrt{\int\left[4 k T R_{F} G_{n p}+4 k T R_{S} G_{n p}^{2}+\left(e_{n} G_{n p}\right)^{2}+\left(i_{n p} R_{S} G_{n p}\right)^{2}+\left(i_{n m} R_{F}\right)^{2}\right] d f} .
\end{aligned}
$$

The integral in Eq. (4.6.18) is evaluated by first moving the enclosed constant terms out of the integral. The resistors and their associated noise are constant over frequency. Therefore the first two terms of Eq. (4.6.18) are constants. The last three terms contain the input referred noise sources of the op-amp. The op-amp's internally generated noise is the combination of flicker, shot, and thermal noise, i.e. white and $1 / f$ noise [35]. 
Assuming that the amplifier's internal current noise sources are balanced, $i_{n p}=i_{n m}=$ $i_{n}$, and the frequency bandwidth $\left(\Delta f=f_{H}-f_{L}\right)$ and frequency response of the network is known. The equivalent noise bandwidth can be determined, i.e. $\Delta f=C_{E N B}\left(f_{H}-f_{L}\right)$, as discussed in section 4.5. Applying Eq. (4.6.9) results in the total mean-squared output voltage noise,

$$
\begin{aligned}
\bar{v}_{o}^{2}= & G_{n p}^{2}\left(\frac{4 k T R_{F}}{G_{n p}}+4 k T R_{S}\right) \Delta f+\left(e_{n} G_{n p}\right)^{2}\left[f_{\text {enc }} \ln \left(\frac{f_{H}}{f_{L}}\right)+\Delta f\right] \\
& +\left(i_{n} G_{n p}\right)^{2}\left[R_{S}^{2}+\left(\frac{R_{F}}{G_{n p}}\right)^{2}\right]\left[f_{i n c} \ln \left(\frac{f_{H}}{f_{L}}\right)+\Delta f\right] .
\end{aligned}
$$

As discussed in section 4.6.1, noise that has a spectral density proportional to $1 / f$ decreases with an increase in frequency. Thus, at higher frequencies noise sources that generate a constant noise spectrum start to dominate, casing Eq. (4.6.19) to reduce to

$$
\bar{v}_{o}^{2}=\left\{\frac{4 k T R_{F}}{G_{n p}}+4 k T R_{S}+e_{n}^{2}+i_{n}^{2}\left[R_{S}^{2}+\left(\frac{R_{F}}{G_{n p}}\right)^{2}\right]\right\} G_{n p}^{2} \Delta f .
$$

Noise figure is a useful figure of merit when comparing different component's noise contributions. The noise factor of a non-inverting op-amp circuit is

$$
\begin{aligned}
F_{o p-a m p} & =\frac{1}{G} \frac{N_{o}}{N_{i}}, \\
& =\frac{\bar{v}_{o}^{2}}{G_{n p}^{2} \bar{v}_{s}^{2}} .
\end{aligned}
$$

Note that the noise factor calculated by Eq. 4.6.21 is a linear quantity. The dB quantity can be calculated by $N F=10 \log (F)$.

\subsubsection{Fully differential operational amplifier noise}

The noise model for the fully differential operational amplifier (FDOA) is constructed by referring its internally generated noise sources to the op-amp input (see Fig. 4.13). The input referred noise current flowing to the FDOA's positive and negative input terminals are $i_{n p}$ and $i_{n m}$ respectively, both currents are in $A / \sqrt{H z}$. Noise sources associated with resistive lumped elements are included in the model [49].

The FDOA circuit analysis is conducted by making use of the superposition principle as described in section 4.6.2. Uncorrelated voltage noise spectral densities add to give the root sum of mean squares (see section 4.4 on adding noise sources).

In order to derive the output noise voltage, the model in Fig. 4.13 is simplified. The source impedance is transformed into its Thévenin equivalent resistance

$$
R_{T H}=\frac{R_{S} R_{T}}{R_{S}+R_{T}}
$$

where the new noise model is given in Fig. 4.14 [49]. Usually FDOA circuits have balanced differential feedback networks by selecting identical resistor values for the inverting and non-inverting feedback networks $R_{F}=R_{F 1}=R_{F 2}$ and $R_{G}=R_{G 1}=R_{G 2}$. Thus the equivalent source impedance towards the inverting and non-inverting inputs of the opamp are given by

$$
R_{G e q}=R_{G}+\frac{R_{T H}}{2} .
$$


The equivalent voltage noise gain of a balanced differential circuit, given in [49] is

$$
G_{n}=\frac{R_{F}}{R_{G e q}} .
$$

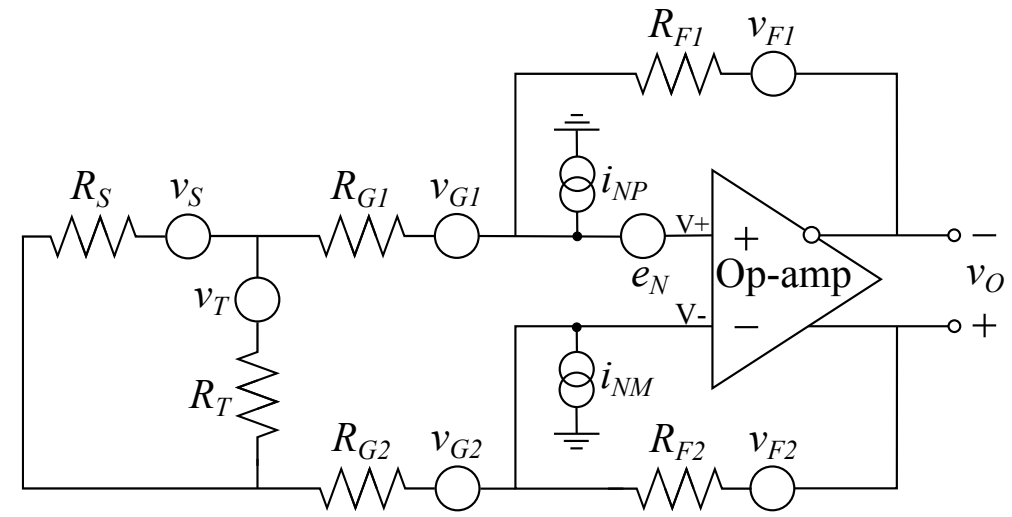

Figure 4.13: Fully differential operational amplifier circuit noise model.

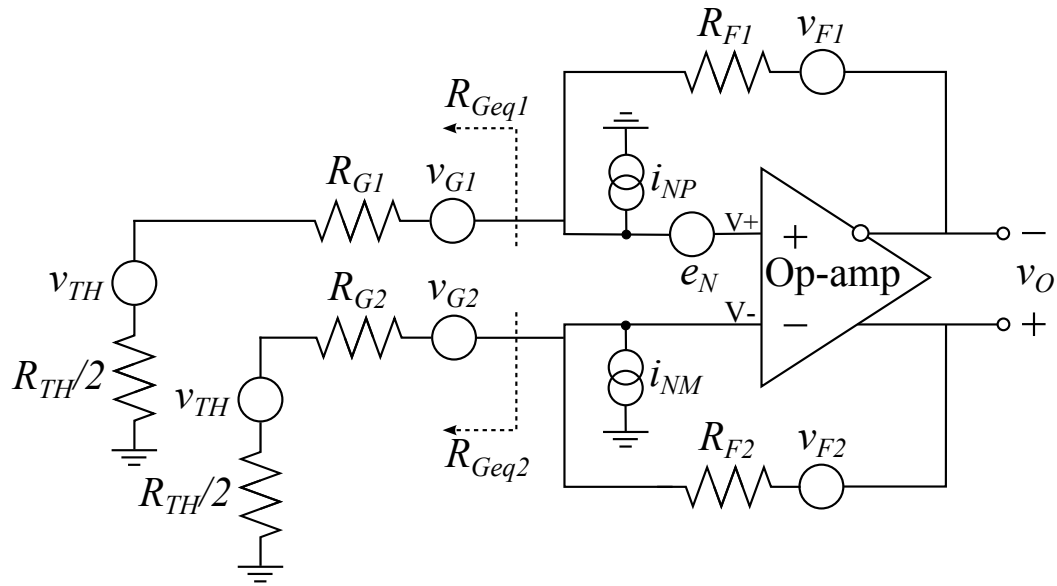

Figure 4.14: Fully differential operational amplifier circuit noise model with Thévenin equivalent input source.

The uncorrelated output referred noise voltages $\left(\bar{v}_{o}\right)$ can be broken into two parts: the thermal noise of the resistive components $\left(\bar{v}_{o r}\right)$ and the noise generated internally by the op-amp $\left(\bar{v}_{o a}\right)$,

$$
\bar{v}_{o}=\sqrt{\bar{v}_{o r}^{2}+\bar{v}_{o a}^{2}} .
$$

The noise generated by the resistive components in the feedback network in Fig. 4.14 is calculated and shown in Table 4.4. The noise generated by these resistors is referred to the output of the FDOA circuit. Adding the noise contributions from the resistive elements in the circuit result in,

$$
\bar{v}_{o r}=\sqrt{2 \bar{v}_{o t h}^{2}+2 \bar{v}_{o g}^{2}+2 \bar{v}_{o f}^{2}} .
$$

Next, the total output referred noise voltage generated internally by the op-amp is calculated, where only uncorrelated noise is taken into account. Thus the current noise $i_{n m}$ and $i_{n p}$ contributions are solely due to the feedback resistor $R_{F}$ [49]. The results are shown in Table 4.5. 
Table 4.4: Fully differential op-amp circuit's feedback network thermal voltage noise

\begin{tabular}{c|c|c}
\hline \hline $\begin{array}{c}\text { Resistive } \\
\text { elements }\end{array}$ & $\begin{array}{c}\text { Source mean-square } \\
\text { voltage noise }\end{array}$ & $\begin{array}{c}\text { Output mean-square } \\
\text { voltage noise }\end{array}$ \\
\hline$R_{T H}$ & $\bar{v}_{t h}^{2}=\int 4 k T \frac{R_{T H}}{2} d f$ & $\bar{v}_{o t h}^{2}=\bar{v}_{t h}^{2} G_{n}^{2}$ \\
$R_{G}$ & $\bar{v}_{g}^{2}=\int 4 k T R_{G} d f$ & $\bar{v}_{o g}^{2}=\bar{v}_{g}^{2} G_{n}^{2}$ \\
$R_{F}$ & $\bar{v}_{f}^{2}=\int 4 k T R_{F} d f$ & $\bar{v}_{o f}^{2}=\bar{v}_{f}^{2}$ \\
\hline
\end{tabular}

Table 4.5: The fully differential op-amp's internal noise sources referred to the output

\begin{tabular}{c|c|c}
\hline \hline $\begin{array}{c}\text { Internal noise } \\
\text { source }\end{array}$ & $\begin{array}{c}\text { Source mean-square } \\
\text { voltage noise }\end{array}$ & $\begin{array}{c}\text { Output mean-square } \\
\text { voltage noise }\end{array}$ \\
\hline$e_{n}$ & $\bar{v}_{n}^{2}=\int e_{n}^{2} d f$ & $\bar{v}_{o n}^{2}=\bar{v}_{n}^{2}\left(1+\frac{R_{F}}{R_{G e q}}\right)^{2}$ \\
$i_{n p}$ & $\bar{v}_{n p}^{2}=\int\left(i_{n p} R_{F}\right)^{2} d f$ & $\bar{v}_{o n p}^{2}=\bar{v}_{n p}^{2}$ \\
$i_{n m}$ & $\bar{v}_{n m}^{2}=\int\left(i_{n m} R_{F}\right)^{2} d f$ & $\bar{v}_{o n m}^{2}=\bar{v}_{n m}^{2}$ \\
\hline
\end{tabular}

Combining the noise sources in Table 4.5 results in the total output referred noise contribution by the FDOA's internal noise generators,

$$
\bar{v}_{o a}=\sqrt{\bar{v}_{o n}^{2}+\bar{v}_{o n m}^{2}+\bar{v}_{o n p}^{2}} .
$$

Lastly the resistive component's and the op-amp's noise sources are combined to get the total output rms noise voltage generated by the op-amp circuit, $\bar{v}_{o}$. As previously stated, it is assumed that the feedback network is balanced. Thus the op-amp's current noise at the positive and negative terminals are effectively equal, $i_{n}=i_{n p}=i_{n m}$.

$$
\begin{aligned}
\bar{v}_{o} & =\sqrt{\bar{v}_{o r}^{2}+\bar{v}_{o a}^{2}}, \\
& =\sqrt{\int\left[8 k T\left(R_{G}+\frac{R_{T H}}{2}\right) G_{n}^{2}+8 k T R_{F}+e_{n}^{2}\left(1+\frac{R_{F}}{R_{G e q}}\right)^{2}+2 i_{n}^{2} R_{F}^{2}\right] d f .}
\end{aligned}
$$

The voltage noise spectral density at the output due to the input signal noise is calculated as follows,

$$
\bar{v}_{o s}^{2}=\int\left[\sqrt{4 k T R_{s}} G_{n}\left(\frac{R_{T}}{R_{S}+R_{T}}\right)\right]^{2} d f,
$$

where $\sqrt{4 k T R_{s}}$ is the voltage noise due to the source equivalent resistor, $G_{n}$ translates the input referred noise voltage to the output, and $\frac{R_{T}}{R_{S}+R_{T}}$ is voltage division due to the $R_{T}$ and $R_{S}$ resistors at the circuit's differential input.

The integrals of Eqs. (4.6.28) and (4.6.29) are evaluated as in section 4.6.2,

$$
\begin{aligned}
\bar{v}_{o}^{2}= & 8 k T\left[R_{G e q} G_{n}^{2}+R_{F}\right] \Delta f \\
& +e_{n}^{2}\left(1+\frac{R_{F}}{R_{G e q}}\right)^{2}\left[f_{\text {enc }} \ln \left(\frac{f_{H}}{f_{L}}\right)+\Delta f\right] \\
& +2 i_{n}^{2} R_{F}^{2}\left[f_{\text {inc }} \ln \left(\frac{f_{H}}{f_{L}}\right)+\Delta f\right],
\end{aligned}
$$


where the bandwidth is either defined by a "brickwall" filter $\Delta f=f_{H}-f_{L}$ or the equivalent noise bandwidth as described in section 4.5. Assuming the op-amp is operating at a high frequency, the pink noise component is negligibly low and Eq. (4.6.30) reduces to

$$
\bar{v}_{o}^{2}=\left[8 k T R_{G e q} G_{n}^{2}+8 k T R_{F}+e_{n}^{2}\left(1+\frac{R_{F}}{R_{G e q}}\right)^{2}+2 i_{n}^{2} R_{F}^{2}\right] \Delta f .
$$

The same procedure is followed when integrating Eq. 4.6.29, resulting in

$$
\bar{v}_{o s}^{2}=\left[\sqrt{4 k T R_{s}} G_{n}\left(\frac{R_{T}}{R_{S}+R_{T}}\right)\right]^{2} \Delta f .
$$

The noise figure of the differential amplifier is calculated in Eq. 4.6.33 by using Eq. 4.3.1. Describing the amplifiers noise in terms of its noise figure is helpful when comparing the noise contributions of different components in a receiver chain.

$$
\begin{aligned}
F_{\text {diff } a m p} & =\frac{1}{G} \frac{N_{o}}{N_{i}} \\
& =\frac{\bar{v}_{o}^{2}}{\bar{v}_{o s}^{2}}
\end{aligned}
$$

\subsection{Noise analysis}

The aim of the noise analysis is to determine the receiver's noise figure, the gain required to lift the voltage noise floor to the lowest effective quantization level of the ADC, and to understand the SFC's (sensitivity frequency control's) impact on the receiver's noise figure.

The noise analysis is conducted in two parts. For the RF front-end components it is assumed that the input and output impedances are equal, thus the available power equivalent models can be used when calculating noise figure. However, this assumption does not hold for all components in the receiver chain. The relationship between the voltage gain and the available power gain of an unmatched network can be described as in Fig. 4.15.

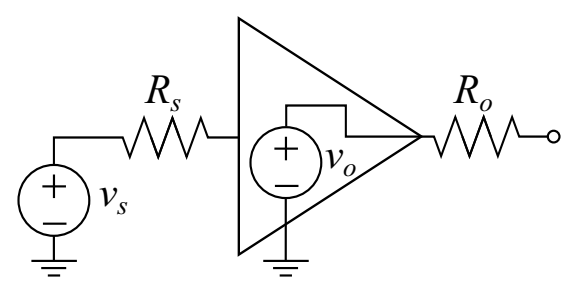

Figure 4.15: System available gain.

Figure 4.15 illustrates an amplifier with output impedance $R_{o}$, driven by a source with an impedance $R_{s}$. For this system, the available power gain can be calculated as,

$$
\begin{aligned}
A_{a} & =\frac{R_{s}}{R_{o}}\left(\frac{v_{o}}{v_{s}}\right)^{2}, \\
G_{a} & =10 \log \left(A_{a}\right) .
\end{aligned}
$$


The voltage gain is given by

$$
\begin{aligned}
A_{v} & =\frac{v_{o}}{v_{s}}, \\
G_{v} & =20 \log \left(A_{v}\right) .
\end{aligned}
$$

When the input and output impedances are equal $\left(R_{s}=R_{o}\right)$ it follows from Eqs. (4.7.1) and (4.7.2) that the voltage and power gain are equal in $\mathrm{dB}\left(G_{a}=G_{v}\right)$. In this case the available power equivalent models can be used to calculate noise figure. By extension if $R_{s} \neq R_{o}$ then $G_{a} \neq G_{v}$, therefore, the available power equivalent models can not be used. The noise figure for the components with unmatched input and output impedances are calculated using the voltage noise and gain as shown in section 4.7.2.

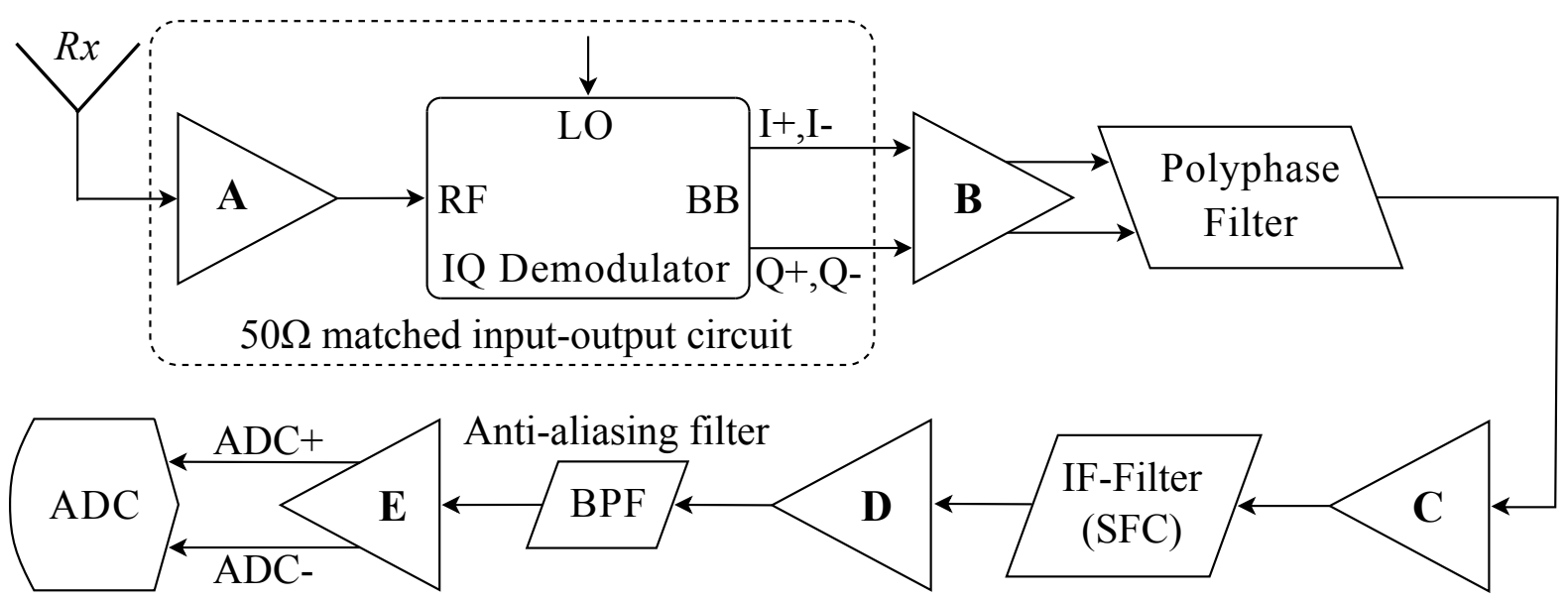

Figure 4.16: Receiver chain block diagram.

Component block A in Fig. 4.16 represents the components matched to $50 \Omega$ at their inputs and outputs. This block includes a one meter section of coaxial transmission line, an LNA, and a RF balun. Fig. 4.17 shows the expansion of block A and the IQ demodulator from Fig. 4.16.

\subsubsection{Matched network noise calculation}

The noise figure of the matched input-output RF components are calculated using the available power model and the equation for cascaded noise figure as described by Eq. 4.3.11. Figure 4.17 shows the matched input-output section of the receiver chain that includes a transmission line, LNA, and RF balun. As discussed in section 4.3.2 it is assumed that a passive component's insertion loss is equal to its noise figure. In practice a one meter long coaxial cable is used to connect the LNA to the input source. The cable has an insertion loss of $0.8 \mathrm{~dB}$ at the FMCW radar's center frequency of $600 \mathrm{MHz}$. The cascaded noise figure of the transmission line, LNA, and balun in Fig. 4.17 is calculated using the values listed in Table 4.6 and 4.7 .

$$
\begin{aligned}
F_{A} & =F_{T L}+\frac{F_{L N A}-1}{G_{T L}}+\frac{F_{\text {balun }}-1}{G_{T L} G_{L N A}}+\frac{F_{\text {demod }}-1}{G_{T L} G_{L N A} G_{\text {balun }}}, \\
& =2.375 \\
N F_{A} & =3.8 \mathrm{~dB} .
\end{aligned}
$$




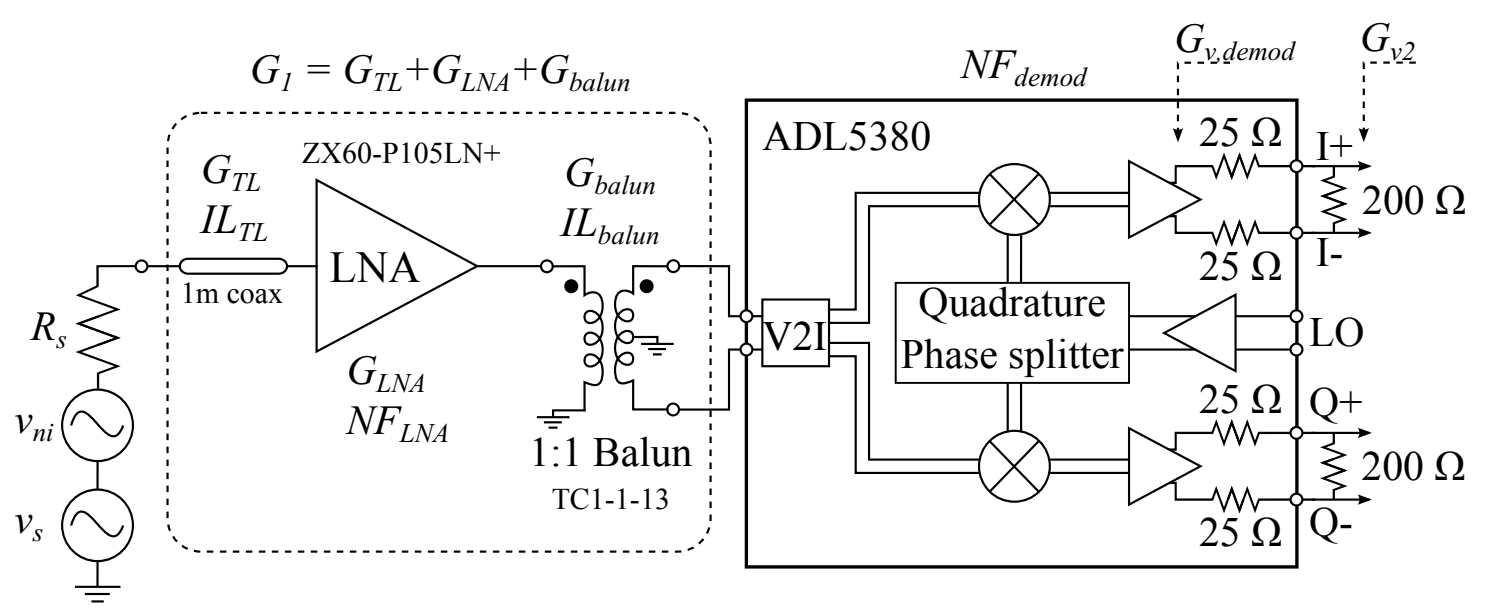

Figure 4.17: RF circuit up to the differential amplifiers at the demodulator output channels I and Q.

Table 4.6: Measured component specifications

\begin{tabular}{c|c|c|c|c}
\hline \hline Component & Symbol & $\begin{array}{c}\text { Gain } \\
{[\mathrm{dB}]}\end{array}$ & $\begin{array}{c}\mathrm{NF} \\
{[\mathrm{dB}]}\end{array}$ & Source \\
\hline One meter coaxial cable & $G_{T L}, N_{T L}$ & -0.8 & 0.8 & Appendix D \\
Analog devices ADL5380 & $G_{v, \text { demod }}$ & -3.4 & - & Section 5.5 \\
\hline
\end{tabular}

Table 4.7: Component specifications from datasheets

\begin{tabular}{c|c|c|c|c}
\hline \hline Component & Symbol & $\begin{array}{c}\text { Gain } \\
{[\mathrm{dB}]}\end{array}$ & $\begin{array}{c}\text { NF } \\
{[\mathrm{dB}]}\end{array}$ & Source \\
\hline Mini-circuits ZX60-P105LN+ & $G_{L N A}, N F_{L N A}$ & 14.5 & 2 & Appendix E, [50] \\
Mini-circuits TC1-1-13 & $G_{\text {balun }}, N F_{\text {balun }}$ & -0.5 & 0.5 & Appendix E, [51] \\
Analog devices ADL5380 & $N F_{\text {demod }}$ & - & 10.7 & {$[15]$} \\
\hline
\end{tabular}

\subsubsection{Noise calculation using voltage noise}

In this section the receiver's noise figure, and the voltage gain required to lift the noise floor to the quantization level of the ADC's least significant bit (LSB) is established.

The voltage equivalent noise model is used to add the noise of the unmatched components in Fig. 4.16 to the noise figure calculation done in the previous section. Components analysed using this model are the: IQ demodulator, differential op-amps B, passive polyphase filter (PPF), op-amp buffer C, passive IF-filter (SFC), and the high voltage gain op-amp D. The calculations are done in $\mathrm{V} / \sqrt{\mathrm{Hz}}$ and the rms voltage noise is determined by integrating over the receiver's bandwidth $(\Delta f=100 \mathrm{kHz})$. It is assumed that the pink noise contribution to the total noise is negligible at the receiver's frequencies of operation, as stated in section 4.6.1. The reactive lumped elements are assumed to have infinitely high $\mathrm{Q}$ values, thus they do not add thermal noise to the system. These assumptions simplify the noise calculation without loosing a great deal of accuracy.

First, the equivalent voltage gain of the circuit in Figure 4.17 is determined. The available power gain, $G_{1}$, is specified under matched conditions, thus in dB the equivalent voltage gain of the transmission line, LNA and balun are equal to the power gain, $G_{1}=G_{v 1}$. The demodulator's open circuit voltage gain $\left(G_{v, \text { demod }}\right)$ is measured and all baluns used during the component's characterisation measurement are de-embedded. The 
demodulator's open circuit voltage gain is defined as the gain from the differential RF input to each of the output baseband channels when terminated in an open circuit. The demodulator's voltage gain when loaded by a $200 \Omega$ resistor is determined from the open circuit gain using voltage division,

$$
\begin{aligned}
A_{v 2} & =10^{G_{v, \text { demod }} / 20} \frac{200 \Omega}{200 \Omega+50 \Omega}, \\
& =0.54 \mathrm{~V} / \mathrm{V} .
\end{aligned}
$$

Equation (4.7.5) describes the equivalent linear voltage gain of the matched components in Fig. 4.17.

$$
\begin{aligned}
A_{v 1} & =10^{G_{v 1} / 20}, \\
& =10^{-0.8 / 20} 10^{14.5 / 20} 10^{-0.504 / 20}, \\
& =4.84 \mathrm{~V} / \mathrm{V} .
\end{aligned}
$$

The total effective voltage gain at the output of the demodulator $\left(A_{v}\right)$ is calculated by multiplying Eq. (4.7.4) and Eq. (4.7.5),

$$
\begin{aligned}
A_{v} & =A_{v 1} A_{v 2}, \\
& =2.62 \mathrm{~V} / \mathrm{V} .
\end{aligned}
$$

Now that the voltage gain of the matched components are known, the equivalent noise voltage at the output of the demodulator in Fig. 4.17 can be determined. Consider the signal-to-noise ratio at the input to the system,

$$
S N R_{i n}=\frac{v_{S}^{2}}{v_{I N}^{2}},
$$

where $v_{S}$ is the input signal voltage, and $v_{N I}$ is the thermal noise voltage due the source impedance $\left(R_{s}=50 \Omega\right)$ as given by Eq. (4.7.8).

$$
\begin{aligned}
& v_{N I}^{2}=k T_{0} R_{s} \\
& v_{N I}=0.45 \mathrm{nV} / \sqrt{\mathrm{Hz}}
\end{aligned}
$$

The output signal-to-noise ratio of the matched components in Fig. 4.16 (block A and IQ demodulator) is,

$$
S N R_{\text {out }, A}=\frac{v_{S}^{\prime 2}}{e_{A}^{2}+v_{N I}^{\prime 2}},
$$

where $v_{S}^{\prime}$ is the signal voltage at the output of the system due to the input signal voltage multiplied by the systems voltage gain. The voltage $e_{A}$ is the noise contribution by the cascaded system, and $v_{N I}^{\prime}$ is the source's voltage noise contribution multiplied by the system's voltage gain as seen at the circuit's output,

$$
v_{N I}^{\prime}=A_{v} v_{N I} \text {. }
$$

The noise figure is calculated as described in section 4.3,

$$
\begin{aligned}
F_{A} & =\frac{S N R_{\text {in }}}{S N R_{\text {out }, A}}, \\
& =\frac{e_{A}^{2}+v_{N I}^{\prime 2}}{A_{v}^{2} v_{N I}^{2}}, \\
& =1+\frac{e_{A}^{2}}{A_{v}^{2} v_{N I}^{2}} .
\end{aligned}
$$


By rearranging Eq. (4.7.11) the voltage noise added by the matched and cascaded components, are given by

$$
\begin{aligned}
e_{A} & =\sqrt{A_{v}^{2} v_{N I}^{2}\left(F_{A}-1\right)}, \\
& =1.38 \mathrm{nV} / \sqrt{\mathrm{Hz}} .
\end{aligned}
$$

Next, the voltage noise at the demodulator's outputs and the noise added by the FDOAs (labelled B in Fig. 4.16) are considered. The FDOA noise analysis is described in section 4.6.3 and the op-amp and resistor values used in this analysis are determined in Chapter 3. The Linear Technologies LT1994 FDOA is analysed for feedback network resistive element values of $R_{F}=82 \Omega$ and $R_{G}=33 \Omega$, where the input Thévenin equivalent source resistor is $R_{T H}=50 \Omega \| 200 \Omega$. The input referred noise sources are illustrated in Fig. 4.18.

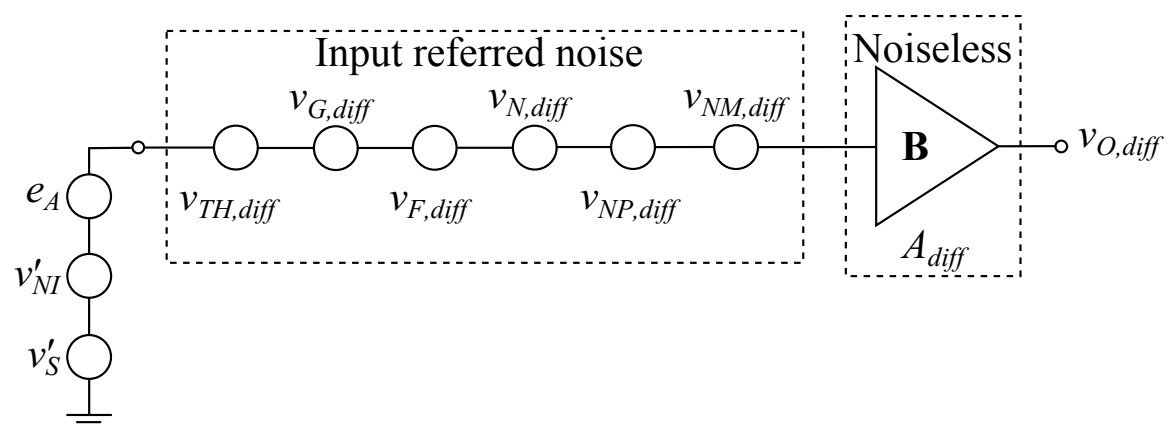

Figure 4.18: Fully differential operational amplifier input and output noise sources.

The voltage noise and signal-to-noise ratio at the op-amp's output is given by Eq. (4.7.13) and Eq. (4.7.14) respectively.

$$
\begin{aligned}
& v_{N O, \text { diff }}^{2}=A_{\text {diff }}^{2}\left(A_{v}^{2} v_{N I}^{2}+e_{A}^{2}+v_{T H, d i f f}^{2}+v_{G, \text { diff }}^{2}\right. \\
& \left.+v_{F, \text { diff }}^{2}+v_{N, \text { diff }}^{2}+v_{N P, \text { diff }}^{2}+v_{N M, d i f f}^{2}\right) \\
& S N R_{\text {out }, \text { diff }}=\frac{A_{\text {diff }}^{2} v_{S}^{\prime \prime 2}}{v_{N O, \text { diff }}^{2}}
\end{aligned}
$$

The receiver's noise figure, with the FDOA included, is determined by Eq. (4.7.15).

$$
\begin{aligned}
F_{\text {diff }} & =\frac{S N R_{\text {in }}}{S N R_{\text {out }, \text { diff }}} \\
& =\frac{v_{N O, \text { diff }}^{2}}{v_{N I}^{2} A_{v}^{2} A_{\text {diff }}^{2}} \\
N F_{\text {diff }} & =13.5 \mathrm{~dB}
\end{aligned}
$$

The influence of the PPF and buffer op-amp C of Fig. 4.16 is now considered. The differential outputs, $\Delta I_{\text {out }}$ and $\Delta Q_{\text {out }}$, are given in terms of the inputs by Eq. (4.7.16). The voltage can be calculated when each output port is loaded by an impedance $Z_{L}[9]$.

$$
\left[\begin{array}{c}
\Delta V_{\text {Iout }} \\
\Delta V_{\text {Qout }}
\end{array}\right]=\frac{Z_{L}}{R+Z_{L}+j \omega C R Z_{L}}\left[\begin{array}{cc}
1 & -j \omega C R \\
j \omega C R & 1
\end{array}\right]\left[\begin{array}{c}
\Delta V_{\text {Iin }} \\
\Delta V_{\text {Qin }}
\end{array}\right]
$$


From Eq. (4.7.16) the voltage gain for both input ports producing an output signal at port three is determined [52],

$$
\begin{aligned}
& A_{v 31}=\frac{Z_{L}}{R+Z_{L}+j \omega C R Z_{L}}, \\
& A_{v 32}=\frac{-j \omega C R Z_{L}}{R+Z_{L}+j \omega C R Z_{L}} .
\end{aligned}
$$
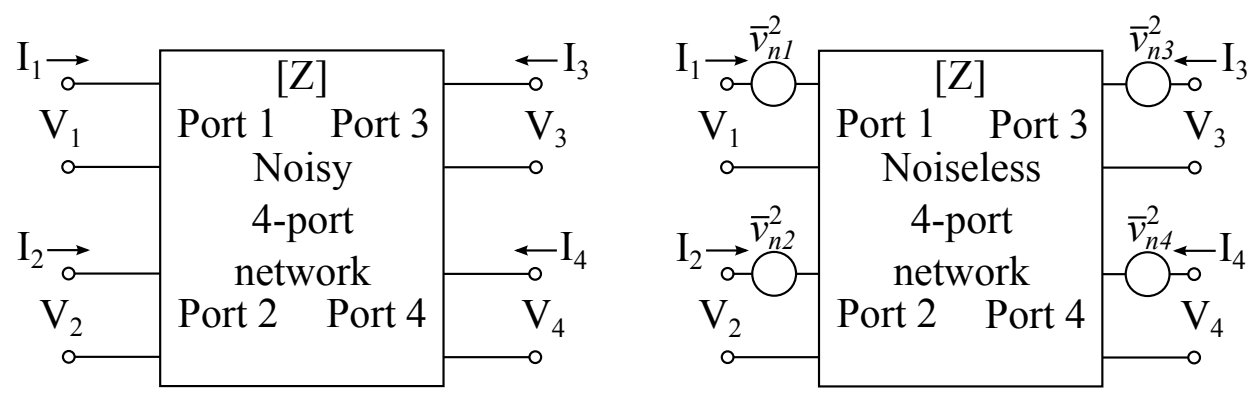

Figure 4.19: Four-port noise representation using Z-parameters.
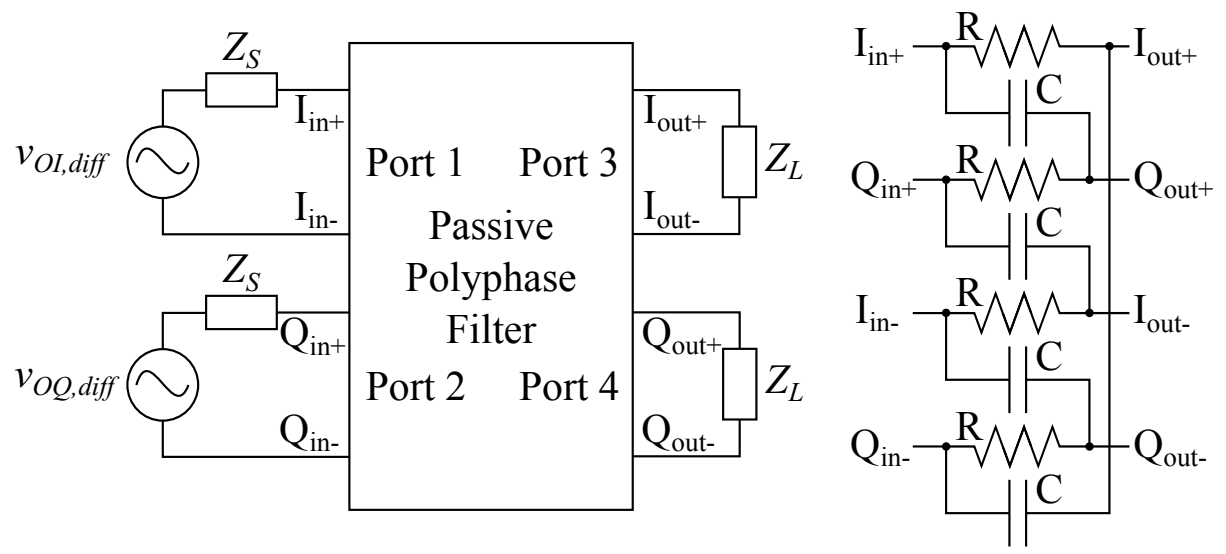

Figure 4.20: Passive polyphase filter four-port network.

Maximum image rejection is realised when the impedance network is balanced, thus the terminating impedances are assumed to be symmetrical. The PPF's input and output ports are buffered by op-amps, so the source and load impedances are approximated by short and open circuits respectively. The noise calculation is conducted at the filter's centre frequency $\omega=\frac{1}{C R}$. As a result of these approximations Eqs. (4.7.17) and (4.7.18) reduce to

$$
\begin{aligned}
A_{v 31} & =\frac{1}{1+j}, \\
\left|A_{v 31}\right| & =\frac{1}{\sqrt{2}}, \\
A_{v 32} & =\frac{-j}{1+j}, \\
\left|A_{v 32}\right| & =\frac{1}{\sqrt{2}} .
\end{aligned}
$$


The forward voltage gain of Fig. 4.19 is defined as,

$$
A_{P P F}=\left|A_{v 31}\right|+\left|A_{v 32}\right| \text {. }
$$

The passive network in Fig. 4.20 is represented by an equivalent Z-parameter matrix,

$$
[Z]=\left[\begin{array}{cccc}
R+1 /(j \omega C) & 0 & 1 /(j \omega C) & R \\
0 & R+1 /(j \omega C) & -R & 1 /(j \omega C) \\
1 /(j \omega C) & -R & R+1 /(j \omega C) & 0 \\
R & 1 /(j \omega C) & 0 & R+1 /(j \omega C)
\end{array}\right]
$$

The voltages and currents at the ports to the network in Fig. 4.19 are represented in terms of Z-parameters as

$$
\left[\begin{array}{l}
V_{1} \\
V_{2} \\
V_{3} \\
V_{4}
\end{array}\right]=[Z]\left[\begin{array}{l}
I_{1} \\
I_{2} \\
I_{3} \\
I_{4}
\end{array}\right]+\left[\begin{array}{l}
V_{n 1} \\
V_{n 2} \\
V_{n 3} \\
V_{n 4}
\end{array}\right]
$$

and the Z-correlation matrix for a four-port network is,

$$
\left[C_{Z}\right]=\left[\begin{array}{cccc}
\left\langle V_{n 1}, V_{n 1}^{*}\right\rangle & \left\langle V_{n 1}, V_{n 2}^{*}\right\rangle & \left\langle V_{n 1}, V_{n 3}^{*}\right\rangle & \left\langle V_{n 1}, V_{n 4}^{*}\right\rangle \\
\left\langle V_{n 2}, V_{n 1}^{*}\right\rangle & \left\langle V_{n 2}, V_{n 2}^{*}\right\rangle & \left\langle V_{n 2}, V_{n 3}^{*}\right\rangle & \left\langle V_{n 2}, V_{n 4}^{*}\right\rangle \\
\left\langle V_{n 3}, V_{n 1}^{*}\right\rangle & \left\langle V_{n 3}, V_{n 2}^{*}\right\rangle & \left\langle V_{n 3}, V_{n 3}^{*}\right\rangle & \left\langle V_{n 3}, V_{n 4}^{*}\right\rangle \\
\left\langle V_{n 4}, V_{n 1}^{*}\right\rangle & \left\langle V_{n 4}, V_{n 2}^{*}\right\rangle & \left\langle V_{n 4}, V_{n 3}^{*}\right\rangle & \left\langle V_{n 4}, V_{n 4}^{*}\right\rangle
\end{array}\right]
$$

By using Eq. (4.7.25) the Z-correlation matrix of a single-sideband spectrum is derived from the Z-parameters of a passive circuit with only thermal noise sources [43] [53].

$$
\left[C_{Z}\right]=4 k T_{0} \Delta f \operatorname{Re}\{[Z]\}
$$

The uncorrelated external voltage noise sources at each of the four-ports to the noiseless network in Fig. 4.19 are determined by Eq. (4.7.26) and described by the diagonal auto-correlated components of the correlation matrix, Eq. (4.7.24).

$$
\bar{v}_{n, P P F}^{2}=4 k T_{0} \Delta f R
$$

Equation (4.7.26) can be defined in units of $\mathrm{V} / \sqrt{\mathrm{Hz}}$ as,

$$
v_{N, P P F}=\sqrt{\frac{\bar{v}_{n, P P F}^{2}}{\Delta f}} .
$$

Although the thermal noise introduced by the PPF's circuitry adds little to the cascaded noise figure, the circuit's voltage gain has a notable influence on the receiver's noise figure.

Figure 4.21 depicts the voltage sources that have an influence on the buffer op-amp C's output. It is worth noting that because port three has a single ended output, the voltage at the port halves. Thus, a voltage gain of $-6 \mathrm{~dB}$ is added at the differential output and the attenuation degrades the final cascaded noise figure of the entire receiver circuit by $0.71 \mathrm{~dB}$. The use of a single output is a design decision based on the fact that implementing a differential filter requires more components and makes tuning more cumbersome.

The voltage noise contributed by the LT6200 op-amp used to buffer the PPF's output (labelled $\mathrm{C}$ in Fig. 4.21) is brought into account as explained in section 4.6.2. The source 


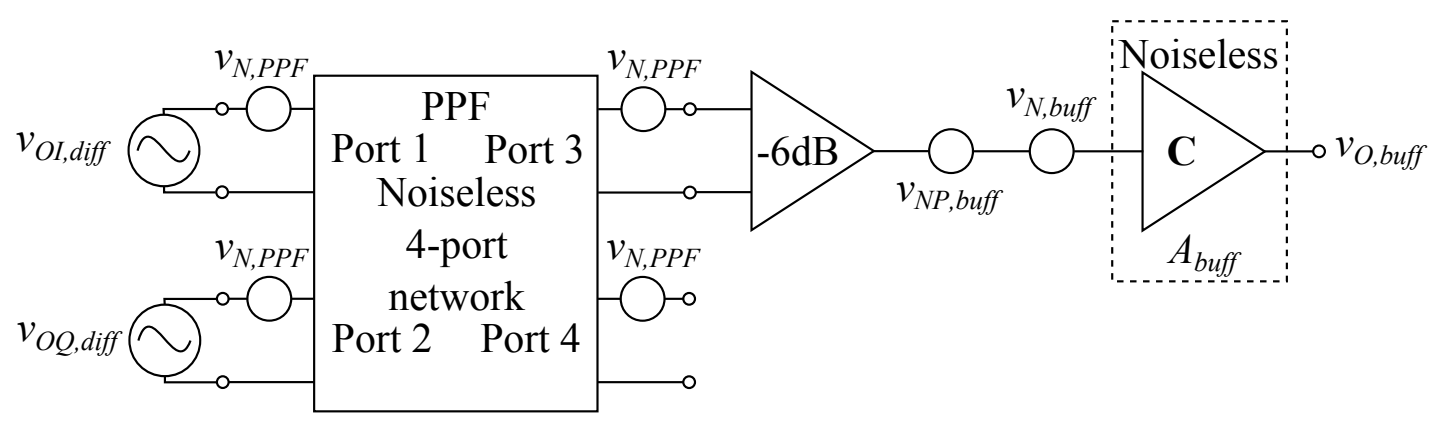

Figure 4.21: Received signal and receiver noise at the output of the buffer op-amp C.

resistance seen by the op-amp is the real part of the output impedance at port three of the four-port network in Fig. 4.20 [9],

$$
Z_{\text {out }}=\frac{j \omega C R Z_{S}+Z_{S}+2 R}{j \omega C Z_{S}+j \omega C R+1} .
$$

Assumed that $Z_{S} \approx 0$ and $Z_{L} \approx \infty$, Eq. (4.7.28) reduces to

$$
R_{S, \text { buff }}=\operatorname{Re}\left\{Z_{\text {out }}\right\}=R,
$$

where a resistance of $75 \Omega$ is used in the PPF network. The noise voltage in units of $\mathrm{V}^{2} / \mathrm{Hz}$ at the output of op-amp $\mathrm{C}$ is determined by Eq. (4.7.30).

$$
\begin{aligned}
V_{N O, b u f f}^{2}= & A_{b u f f}^{2}\left[\left(\frac{1}{2}\right)^{2}\left(A_{P P F}^{2}\left(v_{N O, d i f f}^{2}+v_{N, P P F}^{2}\right)+v_{N, P P F}^{2}\right),\right. \\
& \left.+v_{N P, b u f f}^{2}+v_{N, b u f f}^{2}\right]
\end{aligned}
$$

and the output signal to noise ratio is determined by Eq. (4.7.31).

$$
S N R_{\text {out }, \text { buff }}=\frac{\left(\frac{1}{2}\right)^{2} A_{b u f f}^{2} A_{P F F}^{2} A_{d i f f}^{2} A_{v}^{2} v_{s}^{2}}{v_{N O, b u f f}^{2}}
$$

Inclusion the PPF and buffer op-amp, the cascaded noise figure is determined as

$$
\begin{aligned}
F_{\text {buff }} & =\frac{S N R_{\text {in }}}{S N R_{\text {out }, \text { buff }}}, \\
& =\frac{v_{N O, b u f f}^{2}}{v_{N I}^{2}\left(\frac{1}{2}\right)^{2} A_{b u f f}^{2} A_{P F F}^{2} A_{d i f f}^{2} A_{v}^{2}}, \\
N F_{\text {buff }} & =13.8 \mathrm{~dB} .
\end{aligned}
$$

Now the passive IF-filter's noise contribution is addressed. The LC circuit is represented as an equivalent noiseless network with voltage noise sources at each of its ports, as shown in Fig. 4.22. The available power gain of the network from port 1 to port 2 can be expressed in terms of the scattering parameters of the network and the port mismatches,

$$
G_{21}=\frac{\left|S_{21}\right|^{2}\left(1-\left|\Gamma_{S}\right|^{2}\right)}{\left|1-S_{11} \Gamma_{S}\right|^{2}\left(1-\left|\Gamma_{\text {out }}\right|^{2}\right)}
$$




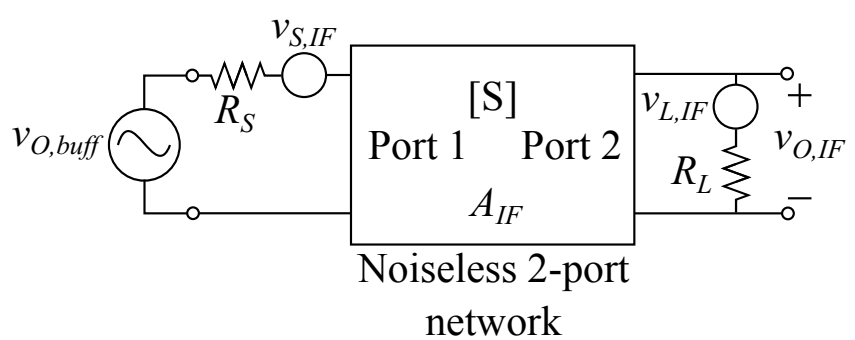

Figure 4.22: IF-filter noise contribution.

For the calculation it is assumed that a perfect input and output match is realised, in other words the insertion loss measured is assumed to be due to component losses and the available voltage gain can be represented as,

$$
A_{I F}^{2}=G_{21}=\left|S_{21}\right|^{2}
$$

The input and output is matched to a $50 \Omega$ impedance and the noise contributed by the source and load resistors are calculated in $\mathrm{V}^{2} / \mathrm{Hz}$ by Eq. (4.7.35).

$$
v_{S, I F}^{2}=v_{L, I F}^{2}=4 k T_{0} R_{50}
$$

The superposition principle is applied to add the voltage noise sources at the output of the network resulting in Eq. (4.7.36),

$$
v_{N O, I F}^{2}=A_{I F}^{2}\left(v_{O, b u f f}^{2}+v_{S, I F}^{2}\right)+v_{L, I F}^{2},
$$

The rms voltage noise in the receiver's bandwidth $(100 \mathrm{kHz})$ at the IF-filter's output is

$$
\begin{aligned}
v_{n o, I F} & =\sqrt{v_{N O, I F}^{2} \Delta f}, \\
& =1.15 \mathrm{uV} .
\end{aligned}
$$

At this point it is possible to determine the voltage gain required to lift the rms voltage noise so that it triggers the ADC's LSB. First, the 12-bit ADC's lowest quantization level is determined at a reference voltage of $3.3 \mathrm{~V}$,

$$
\begin{aligned}
v_{L S B} & =\frac{v_{r e f}}{2^{n}}=\frac{3.3}{2^{12}}, \\
& =0.81 \mathrm{mV} .
\end{aligned}
$$

The minimum voltage gain required from the amplifier, represented by block $\mathrm{D}$ seen in Fig. 4.16, is determined from the ratio between Eqs. (4.7.37) and (4.7.38). Taking into account that the $5 \mathrm{~V}$ supply rail is scaled down to the ADC's reference voltage of $3.3 \mathrm{~V}$, the gain is calculated as

$$
\begin{aligned}
A_{D} & =\frac{v_{L S B}}{v_{n o, I F}} \frac{5 \mathrm{~V}}{3.3 \mathrm{~V}}, \\
& =1063, \\
& =60.5 \mathrm{~dB} .
\end{aligned}
$$

The voltage gain calculated in Eq. (4.7.39) is realised in the receiver circuit by two opamp's in a non-inverting configuration. Each op-amp stage provides a minimum voltage gain of $30.3 \mathrm{~dB}$. The noise analysis on these components is conducted as in section 4.6.2. 
The high gain added at this stage prevents the cascaded noise figure from notable deterioration due to consecutive components in the receiver chain. Consequently the noise figure analysis is conducted up to the output of the high gain stage.

The design voltage gain selected for amplifier D in Fig. 4.16 is $1.9 \mathrm{~dB}$ higher than the value calculated in Eq. (4.7.39). Thus the noise floor is effectively at bit 11.69 of the ADC. Fractional bits are not realisable in practice, but serve as an indication of the rms voltage noise level relative to the ADC's available quantization range. The reason for the additional gain is that the ADC has a finite SNR and also has a signal to noise and distortion (SINAD) ratio. SINAD is converted to effective number-of-bits (ENOB) using the relationship of the theoretical SNR for an ideal $n$-bit ADC [54],

$$
S N R=6.02 n+1.76 \mathrm{~dB} .
$$

Solving for $n$, and substituting the value of SINAD for SNR [54], results in

$$
E N O B=\frac{S I N A D-1.76}{6.02},
$$

where ENOB is a measure of the dynamic performance of an ADC.

The ENOB of the ATMEL SAM3X microcontroller has a typical value of 10.3 bits when used in the differentially mode. Thus, by applying Eq. (4.7.39) it is shown that a voltage gain of $74.6 \mathrm{~dB}$ is required from amplifier D at an ENOB of 10.3 bits. Setting the gain to this value would decrease the receiver's full scale RF input power by $8.4 \mathrm{~dB}$ and utilize less of the ADC's available SNR. (The dynamic range calculation are available in section 5.2.)

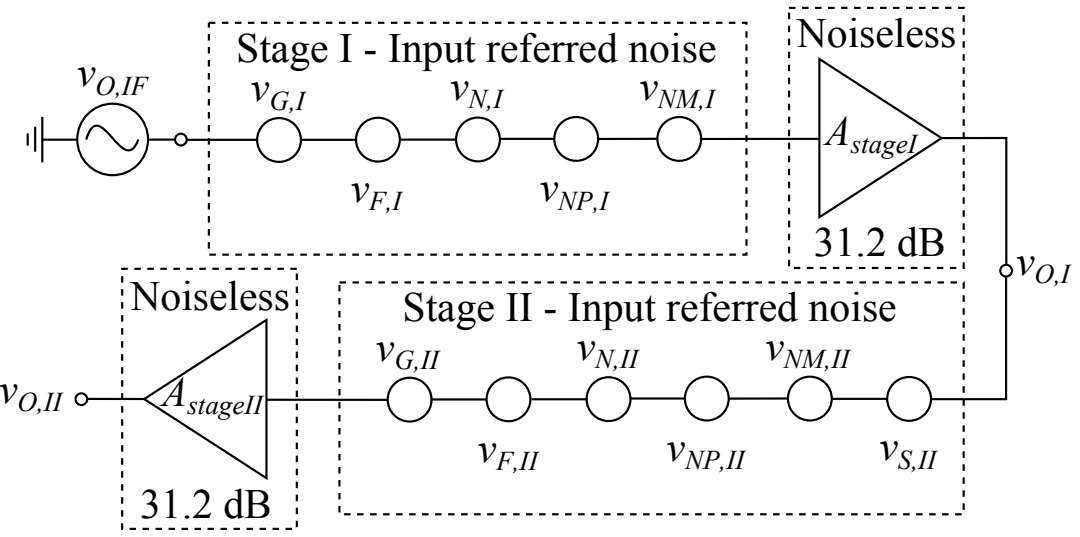

Figure 4.23: The high voltage gain amplifier's noise sources, labelled D in Fig. 4.16.

Amplifier D is constructed using two cascaded LT6200 op-amps, the circuit's noise sources are illustrated in Fig. 4.23. However, the source impedance noise is not shown for the first op-amp stage because its contribution is already included in $v_{O, I F}$. The second op-amp stage has a source resistance of $220 \Omega$ and a Thévenin equivalent input impedance of $194 \Omega$. The input impedance is the source resistance in parallel with resistors $R_{F}$ and $R_{G}$ from stage one's feedback network.

The voltage noise at the output of amplifier D in Fig 4.16 is calculated using Eqs. (4.7.42) and (4.7.43).

$$
\begin{gathered}
v_{N O, I}^{2}=A_{\text {stageI }}^{2}\left(v_{N O, I F}^{2}+v_{G, I}^{2}+v_{F, I}^{2}+v_{N, I}^{2}+v_{N P, I}^{2}+v_{N M, I}^{2}\right) \\
v_{N O, I I}^{2}=A_{\text {stageII }}^{2}\left(v_{N O, I}^{2}+v_{S, I I}^{2}+v_{N M, I I}^{2}+v_{N P, I I}^{2}+v_{N, I I}^{2}+v_{F, I I}^{2}+v_{G, I I}^{2}\right)
\end{gathered}
$$


The output signal-to-noise ratio is

$$
S N R_{\text {out }, \mathrm{cas}}=\frac{\left(\frac{1}{2}\right)^{2} A_{\text {stageII }}^{2} A_{\text {stageI }}^{2} A_{I F}^{2} A_{b u f f}^{2} A_{P F F}^{2} A_{\text {diff }}^{2} A_{v}^{2} v_{s}^{2}}{v_{N O, I I}^{2}}
$$

and the receiver's cascaded noise figure is calculated at the centre frequency of the IFfilter's passband,

$$
\begin{aligned}
F_{\text {cas }} & =\frac{S N R_{\text {in }}}{S N R_{\text {out }, \text { cas }}}, \\
& =\frac{v_{N O, I I}^{2}}{v_{N I}^{2}\left(\frac{1}{2}\right)^{2} A_{\text {stageII }}^{2} A_{\text {stage }}^{2} A_{I F}^{2} A_{\text {buff }}^{2} A_{P F F}^{2} A_{\text {diff }}^{2} A_{v}^{2}}, \\
N F \text { cas } & =14.5 \mathrm{~dB} .
\end{aligned}
$$

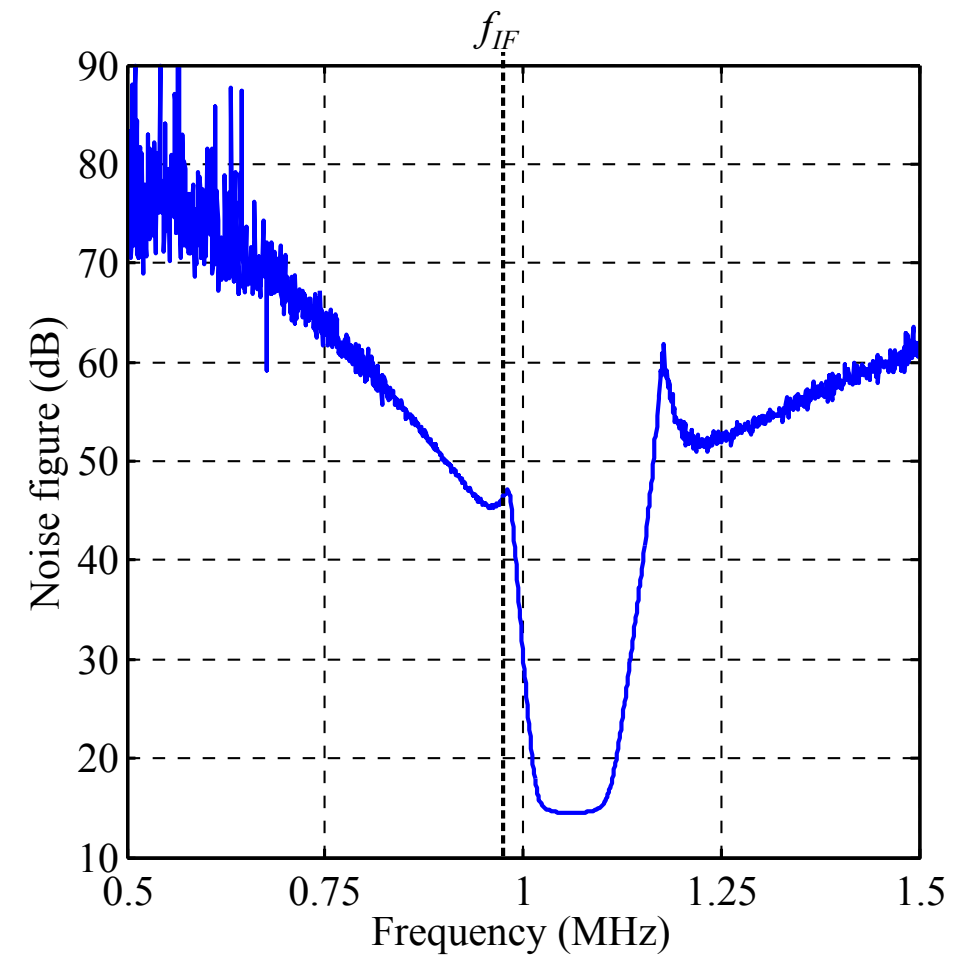

Figure 4.24: Calculated cascaded noise figure versus frequency.

Figure 4.24 illustrates that the cascaded noise figure increases with an increase in IFfilter attenuation. The SFC has a narrow band of operation, $0.976 \mathrm{MHz}$ to $1.036 \mathrm{MHz}$, and the receiver sensitivity is decreased at these operating frequencies. The measured noise figure is presented in section 7.1 and compared to the calculated values.

\subsection{Conclusion}

This chapter considers the effect noise has on an IF FMCW radar receiver. The cascaded gain required to lift the rms voltage noise above the ADC's LSB was determined. The cascaded noise figure was calculated up to the point in the receiver chain where subsequent 
components do not have a notable effect on the noise figure. Finally, the impact SFC has on the receiver's noise figure is investigated. Although the noise figure increases in the SFC bandwidth, the received signals are large and detectable even with decreased receiver sensitivity. 


\section{Chapter 5}

\section{Receiver non-linearity}

In this chapter the representation of non-linearities in RF systems is discussed. Receiver non-linearity produces additional undesired frequency components. To effectively design $\mathrm{RF}$ systems that prevent and remove distortions, the magnitude and frequency of spurious target signals should be predicted [6]. It is proposed that an IF receiver can be designed so that most harmonics and intermodulations products (IMPs), generated due to component non-linearity, are moved beyond the receiver's frequency band of interest.

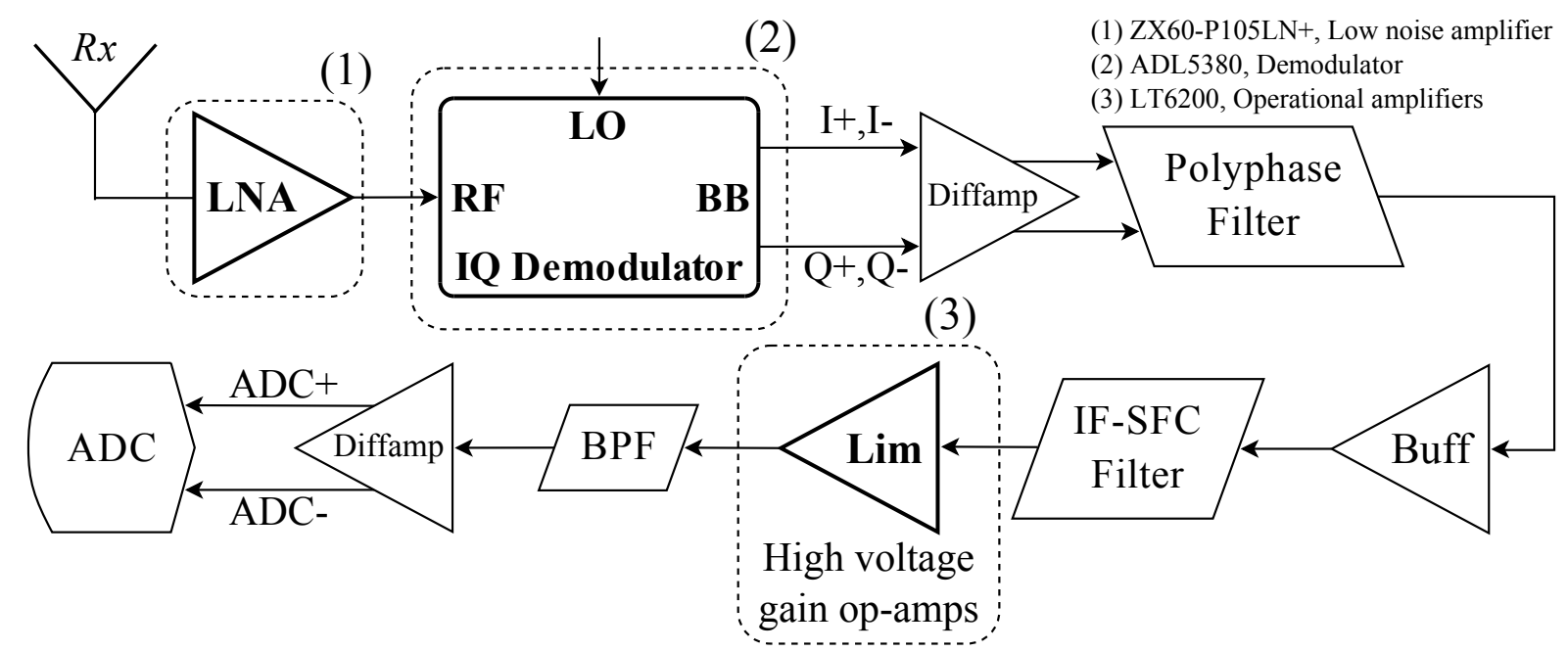

Figure 5.1: FMCW radar IF receiver block diagram.

Receiver saturation can be avoided by knowing the exact nature of the received signals and the characteristics of the receiver. However, in most practical situations there are uncertainties with regards to the received signals.

As it stands there are many methods used to avoid component saturation. Automatic gain control (AGC) can be used to ensure saturation does not occur. Usually for monostatic FMCW radars the designer has control over the transmitter and receiver. Thus, if large reflections are received that cause the receiver to saturation, it is possible to turn down the transmission power. This is especially helpful when targets are close to the radar.

Note that this study is not concerned with the prevention of non-linear behaviour but rather the management of spurious target signals after they occur.

The study is focused on three components in the receiver chain: the low noise amplifier (LNA), quadrature (IQ) demodulator, and the receiver's high gain operational amplifiers 
(op-amps). Assuming a large reflection is received these components are at risk of saturation, they are numbered 1 to 3 in Fig. 5.1.

\subsection{Linearity figure of merit}

An ideal linear device is characterised by it's output signal being exactly proportional to the signal at its input. In reality, even seemingly linear electronic devices exhibit some non-linear behaviour. It is possible that the output non-linearities exist below the minimum detectable level of the measurement tool. Therefore, the harmonics and IMPs are undetected and the device appears linear. In addition, at high input signal levels devices become non-linear and the output signal saturates causing gain compression. The noise floor and the onset of gain compression, sets a minimum and maximum bound to the input and output power for which a device is seemingly linear [55].

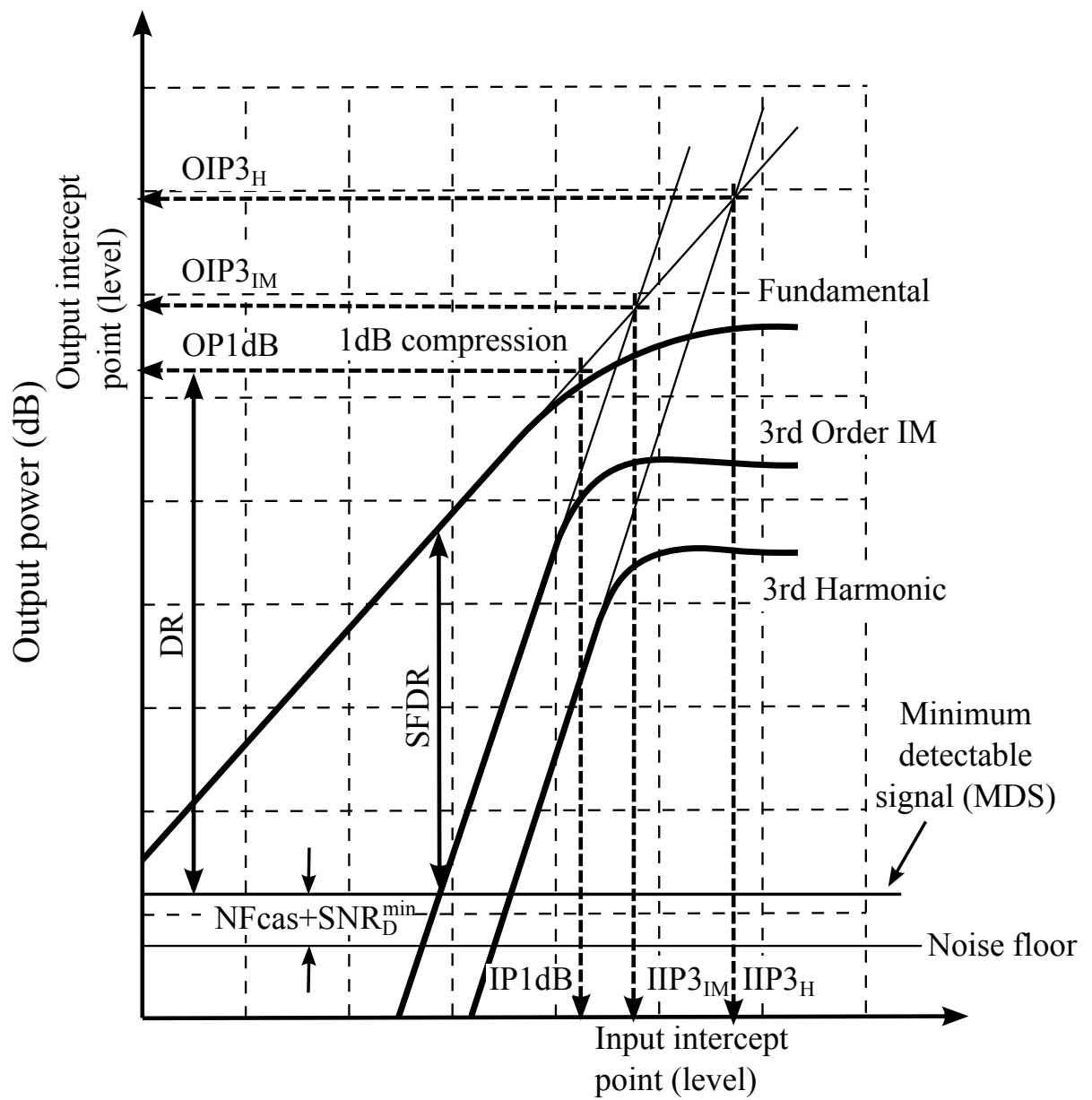

Fundamental input power $(\mathrm{dB})$

Figure 5.2: Graphical representation of dynamic range definitions.

The linearity of a device operating over a narrow frequency band is illustrated in Fig. 5.2, where:

- The input $1 \mathrm{~dB}$ compression point (IP1dB) is defined as the input power at which the power gain decreases by $1 \mathrm{~dB}$ from it's small signal value. OP1dB is the fundamental component's output power corresponding to the input $1 \mathrm{~dB}$ compression point. 
- The input third order intercept point (IIP3) is the power level at which the fundamental signal and the third order intermodulation components (IM3s) would have equal power if no gain compression occurred. (OIP3 is the output third order intercept point corresponding to IIP3.)

- The minimum detectable signal (MDS) level is equal to the input noise floor added to the receiver's cascaded noise figure $\left(N F_{c a s}\right)$ and the minimum required signalto-noise ratio (SNR) at the detector's input. $S N R_{D}^{\min }$ is the SNR that must be exceeded at the ADC's input for a sufficiently accurate quantisation to occur. The MDS level defines the receiver's sensitivity (see Eq. 5.2.4).

- The spurious free dynamic range (SFDR) is defined as the difference between the output power of the fundamental signal and that of the third order IMP when the output IMP crosses the MDS level [55].

- The receiver's dynamic range (DR) is the difference between the output $1 \mathrm{~dB}$ compression point $(\mathrm{OP} 1 \mathrm{~dB})$ and the minimum detectable signal level.

\subsection{Receiver dynamic range}

The last component in the receiver chain is the ADC. An ADC's dynamic range is the difference between the maximum distortion free signal (MDFS) and the minimum detectable signal (MDS) it can reliably quantise at its input.

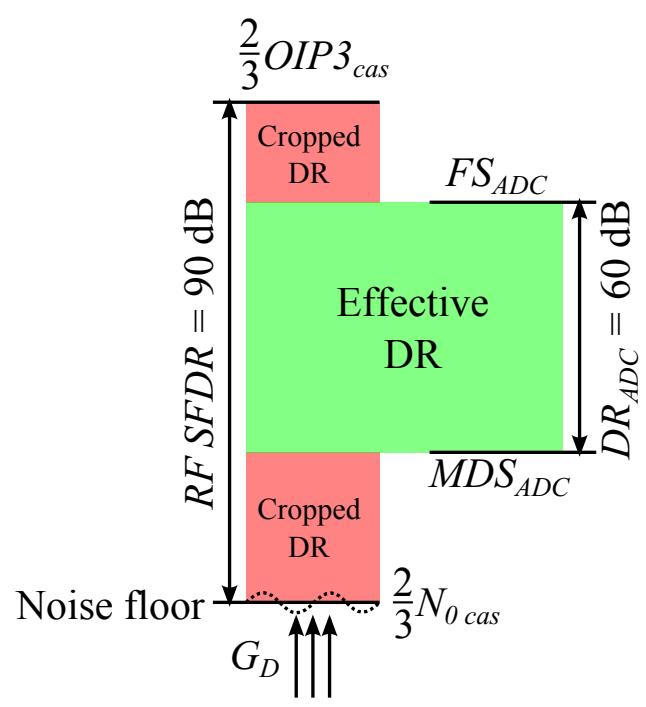

Figure 5.3: Effective dynamic range.

Assuming the cascaded RF front-end component's SFDR (calculated in Chapter 2) is greater than the ADC's dynamic range as in Fig. 5.3. It is found that the ADC truncates the receiver's RF dynamic range. Therefore the ADC's full scale range dictates the largest detectable signal. The smallest signal the ADC can quantise determines the receiver's MDS.

It is desired that after a signal is quantised that the total noise is dominated by the RF front-end's noise contribution. Therefore the first few ADC quantisation steps are occupied by the analog circuit's noise floor. The noise floor is raised by adding gain to the receiver chain [56]. The gain is provided by the high voltage gain op-amps shown in 
Fig. 5.1. The required gain is calculated in section 4.7. The added gain moves the effective dynamic range relative to the total RF SFDR. By adding gain the receiver's sensitivity is increased but its maximum detectable input level is decreased. Consequently, signals that barely rise above the receiver's noise floor are detected and the receiver is more likely to saturate.

Equation (5.2.1) determines the maximum detectable signal at the RF front-end that does not cause the ADC to saturate.

$$
F S_{R X}=F S_{A D C}-G_{c a s}
$$

The ADC's full-scale input power is given by

$$
F S_{A D C}=10 \log _{10}\left(\frac{v_{F S}^{2}}{R_{\text {in }}}\right)+30,
$$

and the full-scale input root mean square (rms) voltage is

$$
v_{F S}=\frac{v_{r e f}}{2 \sqrt{2}}
$$

where $F S_{R X}$ is the full scale RF input power, $G_{c a s}$ is the receiver's conversion gain, $R_{\text {in }}$ is the ADC's input impedance, and $v_{r e f}$ is the ADC's reference voltage.

The minimum detectable signal at the receiver's RF input $\left(M D S_{R X}\right)$ and the ADC's input $\left(M D S_{A D C}\right)$ is determined as follows

$$
\begin{aligned}
M D S_{R X} & =N_{0}+N F_{c a s}+S N R_{D}^{\min } \\
M D S_{A D C} & =M D S_{R X}+G_{c a s}
\end{aligned}
$$

where $N_{0}$ is the thermal noise floor at the RF input in $\mathrm{dBm}$,

$$
N_{0}=10 \log _{10}\left(k T_{o} \Delta f_{R X}\right)+30,
$$

$k$ is the Boltzmann constant, $T_{o}$ the equivalent noise temperature in Kelvin, $\Delta f_{R X}$ is the receiver's frequency bandwidth, and $N F_{c a s}$ is the receiver's cascaded noise figure. The actual noise floor is $3 \mathrm{~dB}$ below the value calculated by Eq. (5.2.5) because the lower sideband is suppressed by the Hartley image rejection architecture. Thus only half the noise power is mixed down to the IF,

$$
N_{0, \text { single sideband }}=N_{0}-3 .
$$

The receiver's detection threshold $\left(S N R_{D}^{\min }\right)$ is the minimum SNR required by the ADC for accurate signal quantisation to occur,

$$
\begin{aligned}
S N R_{D}^{\text {min }} & =10 \log _{10}\left[\frac{\left(\frac{v_{\text {ref }}}{2^{E N O B}}\right)^{2}}{2 R_{\text {in }}}\right]-10 \log _{10}\left[\frac{\left(\frac{v_{r e f}}{2^{n}}\right)^{2}}{2 R_{\text {in }}}\right] \\
& =20 \log _{10}\left[2^{(n-E N O B)}\right] \\
& =1.9 \mathrm{~dB}
\end{aligned}
$$

It is assumed that the noise floor presented to the ADC is equal to the ADC's least significant bit (LSB). The minimum required input signal is dictated by the ADC's effective 
number of bits (ENOB). The difference between Eq. (5.2.1) and Eq. (5.2.4) is equal to the receiver's dynamic range,

$$
\begin{aligned}
D R & =F S_{R X}-M D S_{R X}, \\
& =F S_{A D C}-M D S_{A D C} .
\end{aligned}
$$

Received signals are discretized by the Atmel ATSAM3X8E microcontroller's 12-bit differential ADC. The results shown in Table 5.1 are calculated according to the ADC's specifications as shown in Table 5.2.

The receiver is designed that the high gain op-amp (labelled 3 in Fig. 5.1) saturates before the ADC. The ADC has a quantisation margin of 400 steps (or $0.32 \mathrm{~V}$ ). This prevents non-linearity caused by constructive interference from multi-tone excitation. By comparing the full-scale RF input signal $\left(F S_{R X}=-51.9 \mathrm{dBm}\right.$ see Table 5.1) and the RF front-end's cascaded $1 \mathrm{~dB}$ compression point (IP1dB $=-3.9 \mathrm{dBm}$ see Table 2.5) it is clear that the high gain op-amp is the first device to saturate in the receiver chain.

Table 5.1: Calculated receiver dynamic range

\begin{tabular}{c|c|c|c}
\hline \hline Description & Symbol & Power $[\mathrm{dBm}]$ & Voltage [dBV] \\
\hline Upper sideband noise floor & $N_{0, \text { sideband }}$ & -127 & -140 \\
Full-scale RF signal level & $F S_{R X}$ & -51.9 & -64.9 \\
Full-scale ADC signal level & $F S_{A D C}$ & 5.4 & 1.3 \\
MDS at RF input & $M D S_{R X}$ & -110.1 & -123.1 \\
MDS at ADC input & $M D S_{A D C}$ & -52.8 & -56.8 \\
\hline \hline Dynamic range & $D R$ & \multicolumn{2}{|c}{$58.2 \mathrm{~dB}$} \\
\hline
\end{tabular}

Table 5.2: Variables used in dynamic range calculation

\begin{tabular}{c|c|c|c}
\hline \hline Description & Symbol & Value & Unit \\
\hline Power gain & $G_{c a s}$ & 57.3 & $\mathrm{~dB}$ \\
Voltage gain & $G_{v, c a s}$ & 66.3 & $\mathrm{~dB}$ \\
Noise figure & $N F_{c a s}$ & 14.5 & $\mathrm{~dB}$ \\
ADC resolution & $n$ & 12 & $\mathrm{bits}$ \\
Typical ENOB & $E N O B$ & 11.69 & $\mathrm{bits}$ \\
ADC reference voltage & $v_{r e f}$ & 3.3 & $\mathrm{~V}$ \\
IF bandwidth & $\Delta f_{R X}$ & 100 & $\mathrm{kHz}$ \\
Noise temperature & $T_{0}$ & 290 & $\mathrm{~K}$ \\
ADC input impedance & $R_{i n}$ & 390 & $\Omega$ \\
RF input impedance & $R_{0}$ & 50 & $\Omega$ \\
\hline
\end{tabular}

Figure 5.4 illustrates the IF filter's effect on the receiver's sensitivity and dynamic range. The SFC does not significantly alter the receiver's effective dynamic range (green area in fig. 5.4). However, the receiver's sensitivity decreases and its full scale RF input power increases at low beat frequencies. This allows the FMCW radar to receive large reflections caused by close range targets without saturating. The LNA and IQ demodulator's cascaded $1 \mathrm{~dB}$ compression point ( $\mathrm{IP} 1 \mathrm{~dB}$ cas $)$, calculated in section 2.1.3, is the upper limit to the RF component's SFDR. 


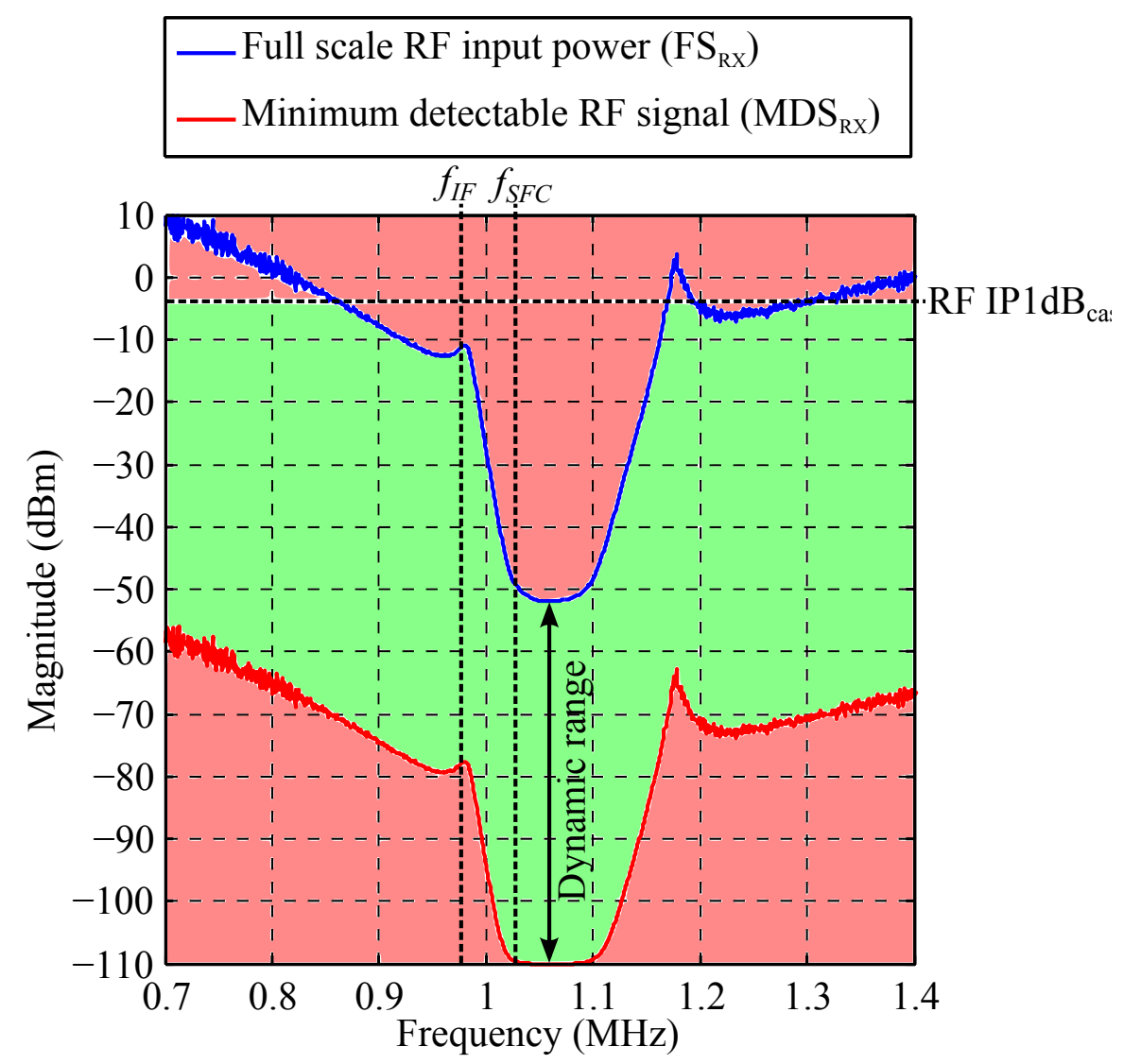

Figure 5.4: Full scale RF input power and minimum detectable RF signal level versus frequency. The cascaded RF $1 \mathrm{~dB}$ compression point $\left(\mathrm{IP} 1 \mathrm{~dB}_{\text {cas }}\right)$ is the upper limit to the cascaded RF component's spurious free dynamic range. The effective dynamic range is the green area between the $F S_{R X}$, RF IP1dB cas and $M D S_{R X}$ lines.

The FS signal level, MDS level, and dynamic range are calculated for a single ideal carrier signal. In reality the dynamic range could be shared amongst multiple carrier signals, i.e. when an FMCW radar receives reflections from multiple targets. Due to constructive interference the full-scale power of the converter is shared among the received signals. Thereby the largest possible singular input for distortion free reception is reduced. Assuming all the received signals are at the same power level, each time the number of carriers double, the largest any of the signals can be is reduced by $3 \mathrm{~dB}$. This is a rough estimate of the adjustment to the FS signal level caused by addition carriers [56]. For example, if four signals are present, each signal must be $6 \mathrm{~dB}$ below the clipping point.

Similar mechanisms exist that cause the noise floor and by extension the MDS to increase. One example is receiver blocking. Blocking is defined as the degradation in a receiver's sensitivity in the presence of a much stronger (blocking) signal. Assuming a desired target signal is monitored at a defined SNR, and a much stronger signal is introduced spectrally near to the desired signal, the effective SNR of the target would degrade. Blocking occurs when the interfering signal is strong enough to cause the receiver's LNA to reach its gain compression point. Causing a decrease in gain and potentially raising the amplifier's noise figure.

Usual in response to a strong input signal the receiver's front-end gain is decreased. However, lowering the gain at the beginning of the receiver chain degrades the receiver's noise figure. The power level of the largest possible reflection is decreased by lowering the transmission power which in turn decreases the radar's maximum range. Range 
performance is directly related to transmission power as shown by Eq. (5.2.9) [1].

$$
R_{\max }=\left[\frac{P_{t} G^{2} \lambda^{2} \sigma}{(4 \pi)^{3} S_{\min }}\right]^{\frac{1}{4}}
$$

Equation (5.2.9) is a simplified form of the radar equation, where $P_{t}$ is transmitted power (watts), $G$ is the antenna gain, $\lambda$ signal wave length, $\sigma$ is a targets radar cross section $\left(m^{2}\right)$, and $S_{\text {min }}$ the minimum detectable signal (watts).

\subsection{Representing non-linear responses}

Device non-linearity can be described by a set of non-linear equations where the output is disproportional to its input. Non-linear equations contain variables with exponents other than one. In other words a device's output versus input plot would ideally result in a straight line extending indefinitely [6]. In practice this graph has some curvature that represents saturation, as illustrated in Fig. 5.5.

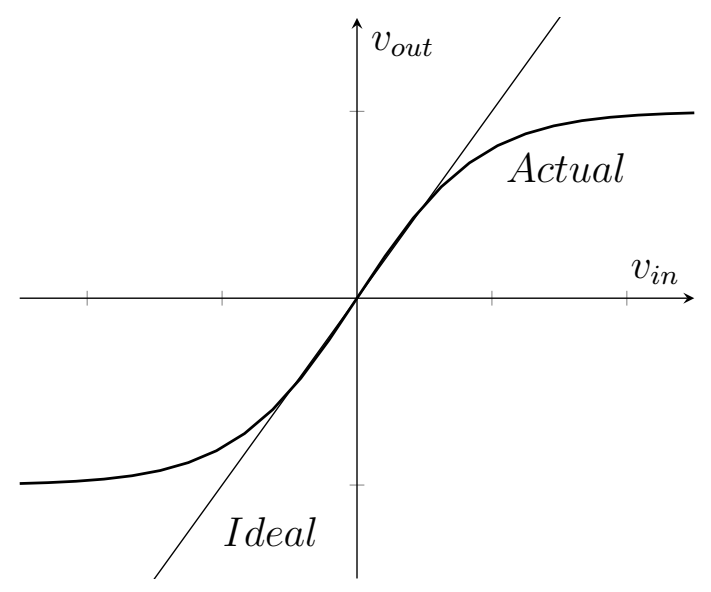

Figure 5.5: Voltage transfer curve with idealized straight line approximation.

An input-output curve can be represented by a Taylor series

$$
f(x)=f\left(x_{0}\right)+f^{\prime}\left(x_{0}\right)\left(x-x_{0}\right)+\frac{f^{\prime \prime}\left(x_{0}\right)}{2 !}\left(x-x_{0}\right)^{2}+\ldots+\frac{f^{n}\left(x_{0}\right)}{n !}\left(x-x_{0}\right)^{n},
$$

or Volterra series depending on the need to capture memory effects. Equation (5.3.1) is the general Taylor series where the variable $x$ is the changing input about the operating point. The operating point is defined as the point about which the series is written. A special case where the series is considered about $x_{0}=0$ is known as the Maclaurin series. The series expansion's coefficients are defined by the expression

$$
a_{n}=\frac{f^{n}\left(x_{0}\right)}{n !} .
$$

In Eq. (5.3.3), $v_{\text {in }}$ is the change in input voltage from the operating point, and the Taylor series coefficients $a_{n}$ are real valued. The first term is a direct current (DC) bias term and is not of interest here. The second term is the desired linear term, where $a_{1}$ 
represents a device's linear gain. The remaining higher order terms are the undesirable components that represent the curvature of the gain curve. If only one signal is present at the input the undesirable components will be harmonics of the fundamental frequency. However, if there are more than one input carrier additional signals at frequencies that are mathematical combinations of the fundamental frequencies of the input carriers are produced. These combinations are called intermodulation products (IMPs) [6]. To gain further insight without complicating the algebra a two input carrier case is considered.

$$
v_{\text {out }}=a_{0}+a_{1} v_{\text {in }}+a_{2} v_{\text {in }}^{2}+a_{3} v_{\text {in }}^{3}+\ldots+a_{n} v_{\text {in }}^{n}
$$

We write the general input signal as

$$
\begin{aligned}
v_{i n}(t) & =A_{1} \cos \left(\omega_{1} t+\theta_{1}\right)+\cdots+A_{M} \cos \left(\omega_{M} t+\theta_{M}\right), \\
& =A_{1} \cos \left[\phi_{1}(t)\right]+\cdots+A_{M} \cos \left[\phi_{M}(t)\right] \quad \text { for } M \geq 1, M \in \mathbb{N},
\end{aligned}
$$

and the two carrier input is represented as

$$
v_{i n}(t)=A_{1} \cos \left[\phi_{1}(t)\right]+A_{2} \cos \left[\phi_{2}(t)\right]
$$

To keep the equations succinct the explicitly written time dependencies may be dropped, writing $\phi$ for $\phi(t)$.

\subsubsection{Odd order terms}

The odd order terms in Eq. (5.3.3) add to the third order IMPs and harmonic. These spurious components are of interest in the analysis of system non-linearities because they have large magnitudes and are spectrally near to the fundamental signals, making it difficult to remove them by filtering. If these unwanted signals are generated and have great enough magnitude they are discretized by the ADC and consequently interpreted as false targets.

To gain further insight into the character of non-linear devices we truncate the infinite Taylor series to only the third term $\left(a_{3} v_{i n}^{3}\right)$ in Eq. (5.3.3). The third term describes the frequency interaction of the fundamental components and adds to the magnitude of the third order distortions and fundamental components. At this point we substitute Eq. (5.3.5) into the third term of Eq. (5.3.3) resulting in a trigonometric polynomial. Employing the appropriate trigonometric identities Eq. (5.3.8) is obtained from Eq. (5.3.7) [6],

$$
\begin{gathered}
a_{3} v_{i n}^{3}=a_{3}\left[A_{1} \cos \left(\phi_{1}\right)+A_{2} \cos \left(\phi_{2}\right)\right]^{3} \\
=a_{3}\left\{\begin{array}{c}
A_{1}^{3} \cos ^{3} \phi_{1}+A_{1}^{2} \cos ^{2} \phi_{1} A_{2} \cos \phi_{2} \\
+2 A_{1}^{2} \cos ^{2} \phi_{1} A_{2} \cos \phi_{2}+2 A_{1} \cos \phi_{1} A_{2}^{2} \cos ^{2} \phi_{2} \\
+A_{2}^{3} \cos ^{3} \phi_{2}+A_{2}^{2} \cos ^{2} \phi_{2} A_{1} \cos \phi_{1}
\end{array}\right\} \\
=\frac{a_{3}}{4}\left\{\begin{array}{c}
\left(3 A_{1}^{3}+6 A_{1} A_{2}^{2}\right) \cos \phi_{1}+\left(3 A_{2}^{3}+6 A_{1}^{2} A_{2}\right) \cos \phi_{2} \\
+3\left[A_{1}^{2} A_{2} \cos \left(2 \phi_{1}-\phi_{2}\right)+A_{1} A_{2}^{2} \cos \left(2 \phi_{2}-\phi_{1}\right)\right] \\
+3\left[A_{1}^{2} A_{2} \cos \left(2 \phi_{1}+\phi_{2}\right)+A_{1} A_{2}^{2} \cos \left(2 \phi_{2}+\phi_{1}\right)\right] \\
+A_{1}^{3} \cos \left(3 \phi_{1}\right)+A_{2}^{3} \cos \left(3 \phi_{2}\right)
\end{array}\right\}
\end{gathered}
$$

where $\phi_{1} \equiv \phi_{1}(t)$ and $\phi_{2} \equiv \phi_{2}(t)$. 
The first line in Eq. (5.3.8) contain signals at the fundamental frequencies, but their amplitudes are non-linear functions of the input amplitudes. They will add to the nonlinear shape of the fundamental gain at high input levels. The second and third lines are the intermodulation terms while the last line in Eq. (5.3.8) adds to the third order harmonics.

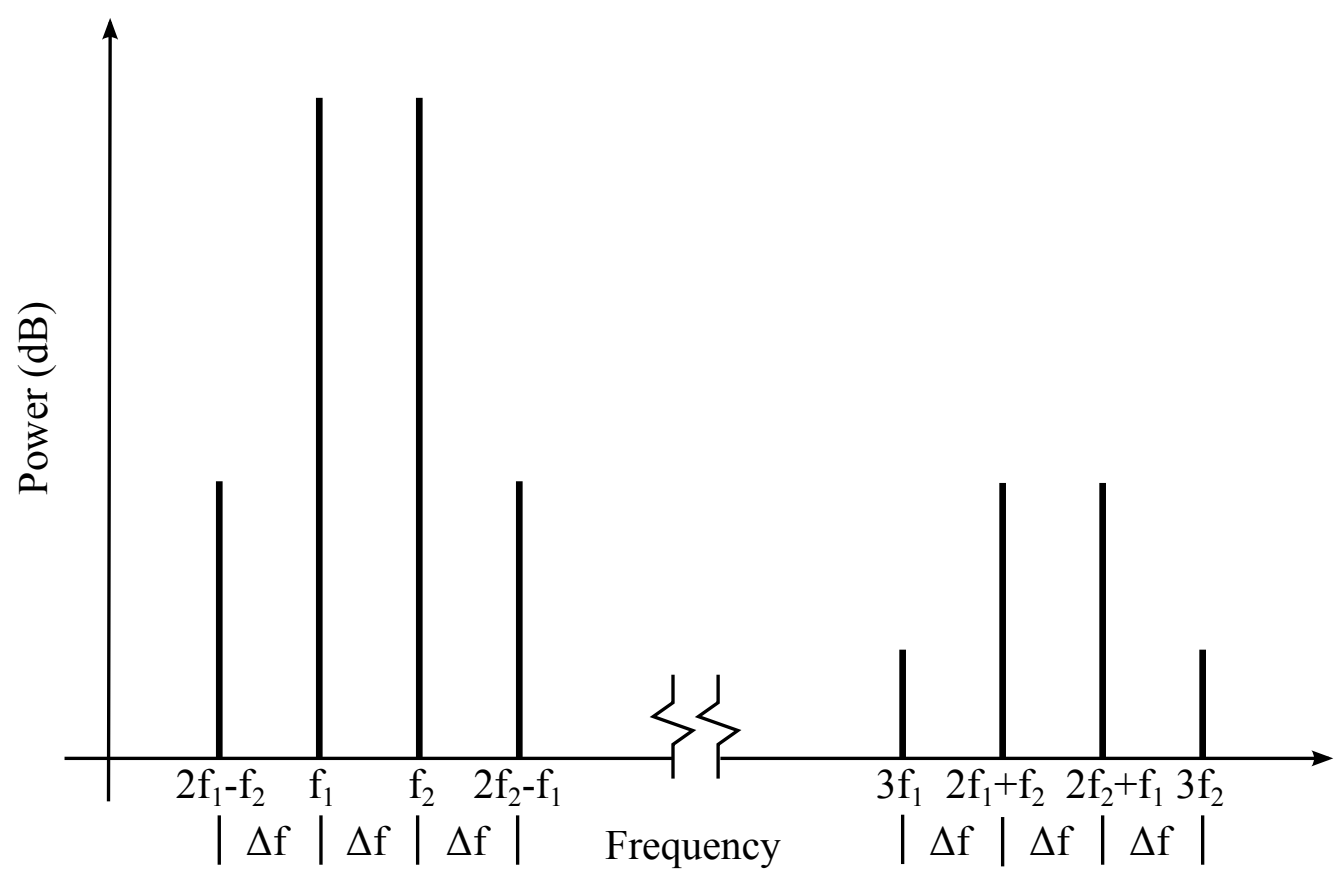

Figure 5.6: Spectrum of third order products from two equal-power inputs.

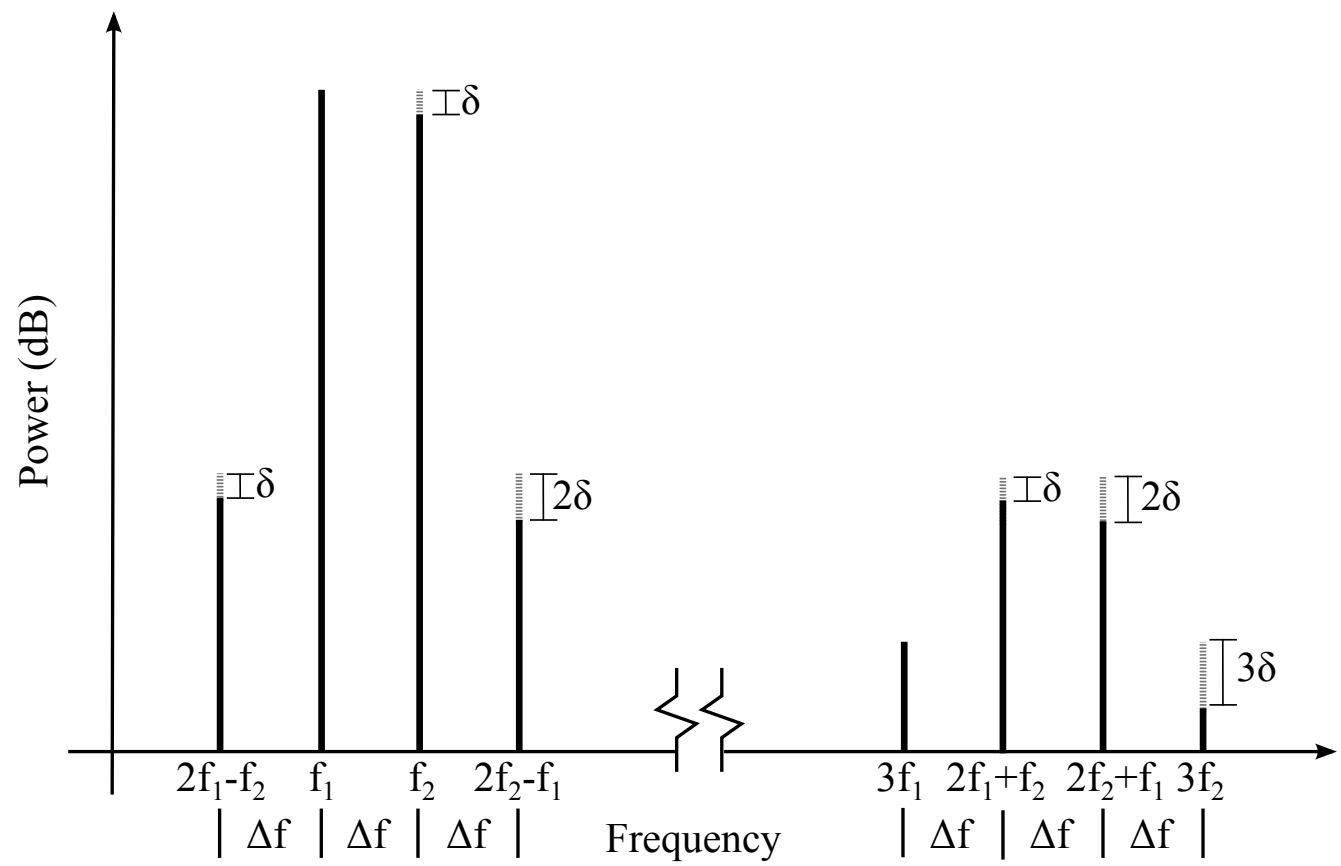

Figure 5.7: Spectrum of third order products from two unequal-power inputs, where $\delta$ represents the difference in amplitude of the fundamental components. 
At low input power levels IMPs and harmonics that contain $n$ times a frequency have amplitudes that are proportional to the $n$th power of the corresponding fundamental amplitude. At high input power levels, other terms with powers of $n+2 k$, where $n$ and $k$ are integers, become appreciable and produce curvature in the response plot [6]. In other words the device under test (DUT) becomes more non-linear with greater input power. Thus more terms of the series expansion is required to accurately approximate the device non-linearity when the device is deeper in saturated.

Figure 5.6 shows the truncated third order frequency spectrum with two inputs of the same magnitude $\left(A_{1}=A_{2}\right)$. The change in the spectrum due to the difference in input level of the fundamental signals is illustrated by Fig. 5.7.

\subsubsection{Intercept points}

The deviation by the fundamental component from its ideal straight-line output is represented by Fig. 5.2. At low input levels the output harmonics and IMPs have a linear progression. Consider the ideal case with no output compression, the third order products increases thrice as fast as the desired fundamental. At some point these straight lines cross and the spurious-and-fundamental signals have equal output power. Since the slopes of the straight lines are known, these crossing points, called intercept points (IPs), define the third order products at low levels. The power out at the third order IP is called the third order intermodulation output intercept point and is represented by the shorthand OIP3 ${ }_{I M}[6]$. For the input power where the harmonic curve crosses the fundamental, the term would be IIP $3_{H}$. Since the IP lies on the linear response curve, an OIP is higher than the corresponding IIP by the linear gain.

From Eq. (5.3.8) the harmonic output power $\left(p_{\text {out }, H 3}\right)$ is determined [6], by relating the fundamental component's output power $\left(p_{\text {out }, F}\right)$ and the third order harmonic output intercept point $\left(\mathrm{OIP} 3_{H}\right)$ as follows

$$
p_{\text {out }, H 3}=\frac{p_{\text {out }, F}^{3}}{p_{O I P 3, H}^{2}} .
$$

The IMP's output power $\left(p_{\text {out }, I M 3}\right)$ at frequency $2 f_{1} \pm f_{2}$ is related to the power of the two fundamental outputs $\left(p_{\text {out }, F 1}\right.$ at $f_{1}, p_{\text {out }, F 2}$ at $\left.f_{2}\right)$ and the third order IM intercept point $\left(\mathrm{OIP} 3_{I M}\right)[6]$,

$$
p_{\text {out }, I M 3}\left(2 f_{1} \pm f_{2}\right)=\frac{p_{\text {out }, F 1}^{2} p_{\text {out }, F 2}}{p_{\text {OIP } 3, I M}^{2}} .
$$

The same holds for the IMP at $2 f_{2} \pm f_{1}$,

$$
p_{\text {out }, I M 3}\left(2 f_{2} \pm f_{1}\right)=\frac{p_{\text {out }, F 2}^{2} p_{\text {out }, F 1}}{p_{\text {OIP } 3, I M}^{2}} .
$$

From Eqs. (5.3.10) and (5.3.11) it is seen that if the two fundamental signal's power levels $P_{\text {out }, F 1}$ and $P_{\text {out }, F 2}$ are equal the third order IMPs are also equal.

\subsection{Low noise amplifier non-linearity}

The Mini-Circuits ZX60-P105LN+ LNA's non-linear behaviour is measured by applying a range of signal power levels at its input while measuring its output port. 
For the measurement a Rohde \& Schwarz (R\&S) SMIQ04B signal generator applies input signals ranging from -90 to $16 \mathrm{dBm}$ at a fixed frequency of $400 \mathrm{MHz}$. The output signal's spectral content is measured by an R\&S FSEK30 spectrum analyser. The measurement is set-up as shown in Fig. 5.8.

Note that signal generators are not ideal linear signal sources. The R\&S SMIQ04B signal generator produces harmonics up to $-30 \mathrm{dBc}$ at output levels of $\leq 7 \mathrm{dBm}$ [57]. However, for accurate measurements a more linear input signal is required that only distortions generated by the LNA is detected by the spectrum analyser. The Mini-Circuits SLP-450 low pass filter is used to suppress unwanted harmonics before the RF signal is applied to the LNA's input.

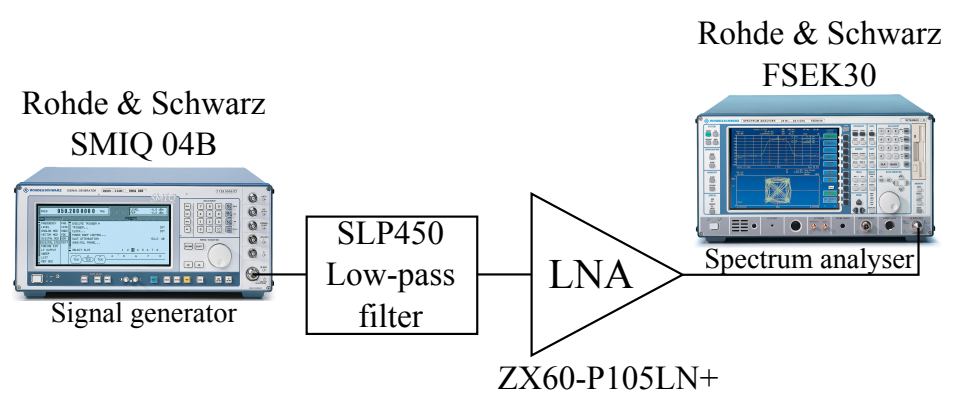

Figure 5.8: LNA measurement set-up.

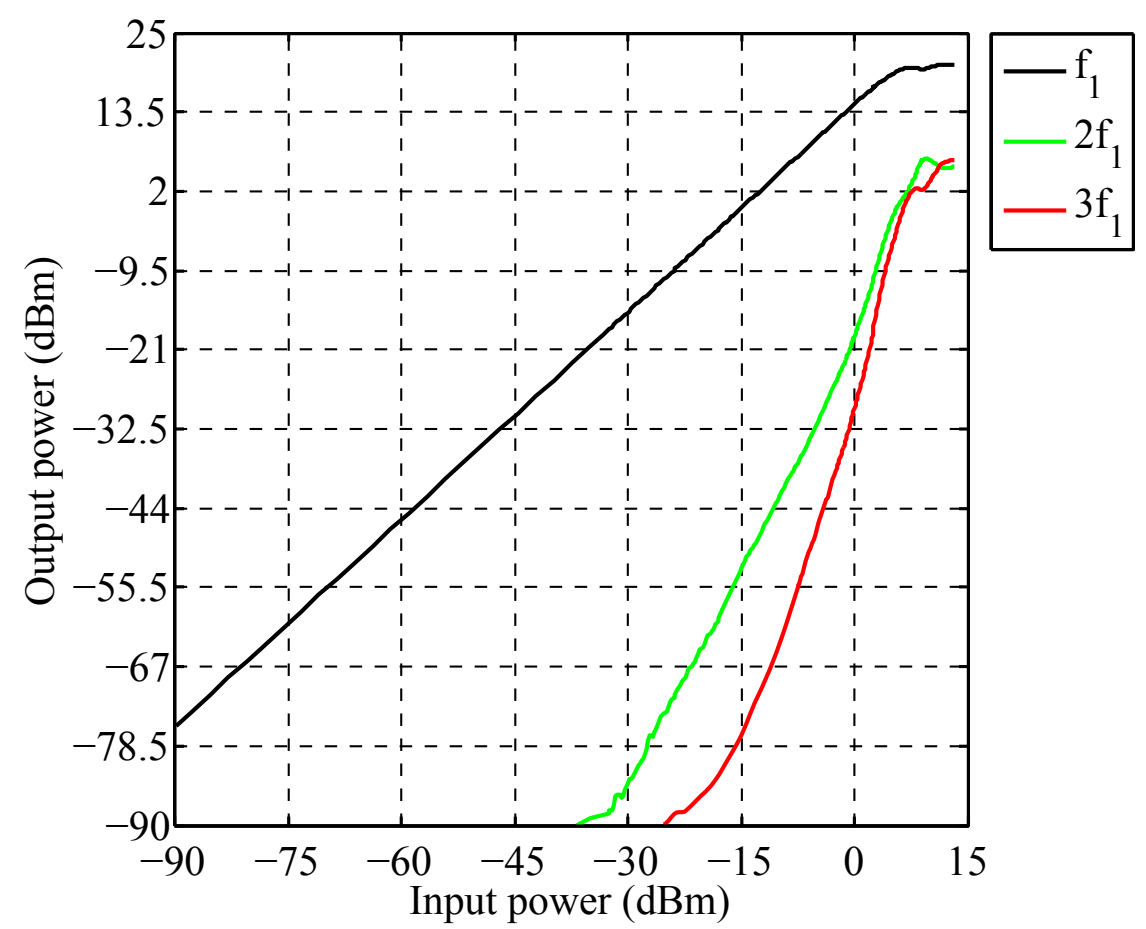

Figure 5.9: The Mini-Circuits ZX60-P105LN+ LNA's output harmonics versus input power.

From Fig. 5.9 the LNA's input $1 \mathrm{~dB}$ compression point is determined after deembedding all cable and filter losses from the data. Its S-parameters are measured using a vector network analyser and presented in Fig. 5.10. The linear power gain is calculated by taking the mean value of $\left|S_{21}\right|$. The results are presented in Table 5.3. 
At this point in the receiver chain the harmonics generated by an LNA can be removed by both the baseband (BB) and IF receiver topologies. Thus no advantage is gained from using an IF receiver at this stage.

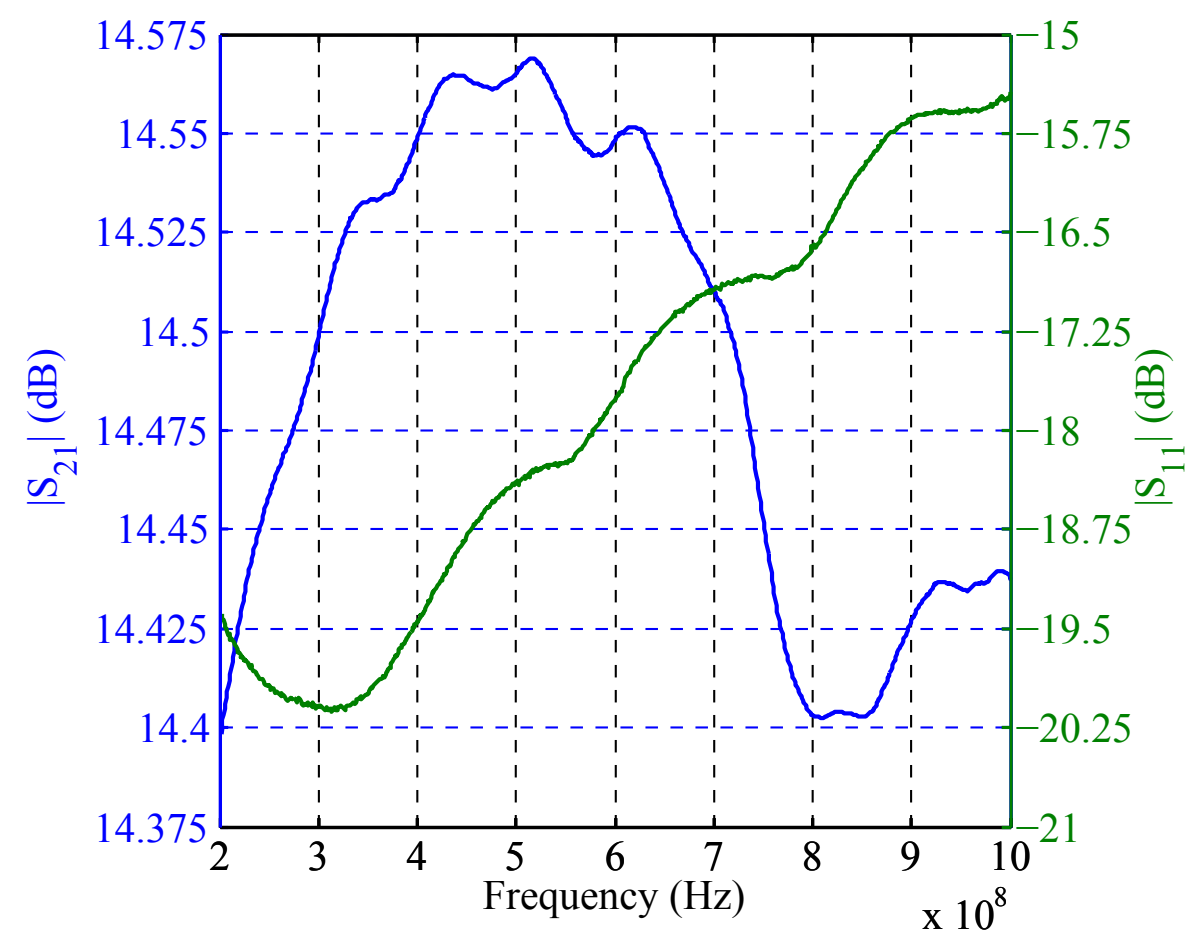

Figure 5.10: Mini-Circuits ZX60-P105LN+ LNA s-parameters.

Table 5.3: Measured ZX60-P105LN+ LNA parameter

\begin{tabular}{c|c|c}
\hline \hline Parameter description & Symbol & Value \\
\hline Input 1 dB compression point & IP1dB & $5.7 \mathrm{dBm}$ \\
Linear power gain & $G_{L N A}$ & $14.4 \mathrm{~dB}$ \\
\hline
\end{tabular}

\subsection{Quadrature demodulator non-linearity}

The Analog Devices ADL5380 quadrature demodulator's non-linearity is accessed by measuring the harmonics generated by a single $\mathrm{RF}$ input tone at a range of power levels.

The measurement is set-up as illustrated in Fig. 5.11, where the R\&S SML03 signal generator supplies a fixed LO of $-3 \mathrm{dBm}$ at $399 \mathrm{MHz}$. The RF input power level (supplied by the R\&S SMIQ 04B signal generator) ranges from -80 to $14 \mathrm{dBm}$ at $400 \mathrm{MHz}$. Therefore, the down converted IF carrier signal is at $1 \mathrm{MHz}, f_{I F}=f_{R F}-f_{L O}$.

The Mini-Circuit's TCM9-1 transformer presents a single ended output from the baseband I-channel to the spectrum analyser's $50 \Omega$ input. The Q-channel is also terminated through a balun in a $50 \Omega$ load. A $50 \Omega$ single ended load results in a $450 \Omega$ differential impedance at the I and $\mathrm{Q}$ channels when using a 9:1 impedance transformer. A decoupling capacitor $(0.1 \mathrm{uF})$ is connected to the centre tap of the transformer to reject noise at the demodulator output. 


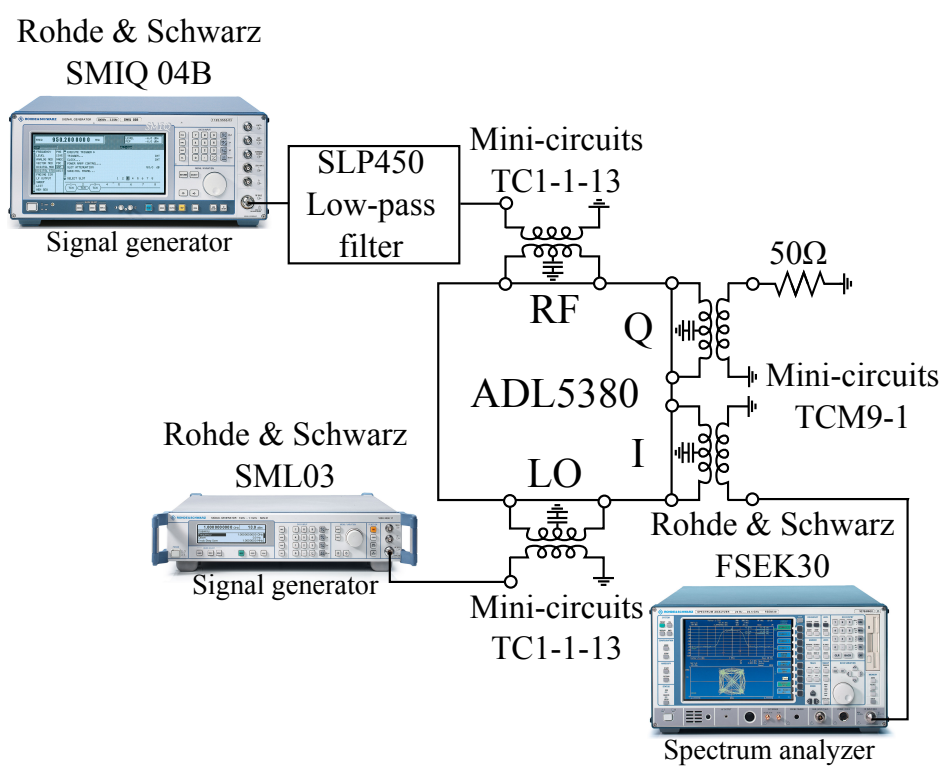

Figure 5.11: The set-up used to measure the Analog Devices ADL5380 quadrature demodulator's non-linear behaviour.

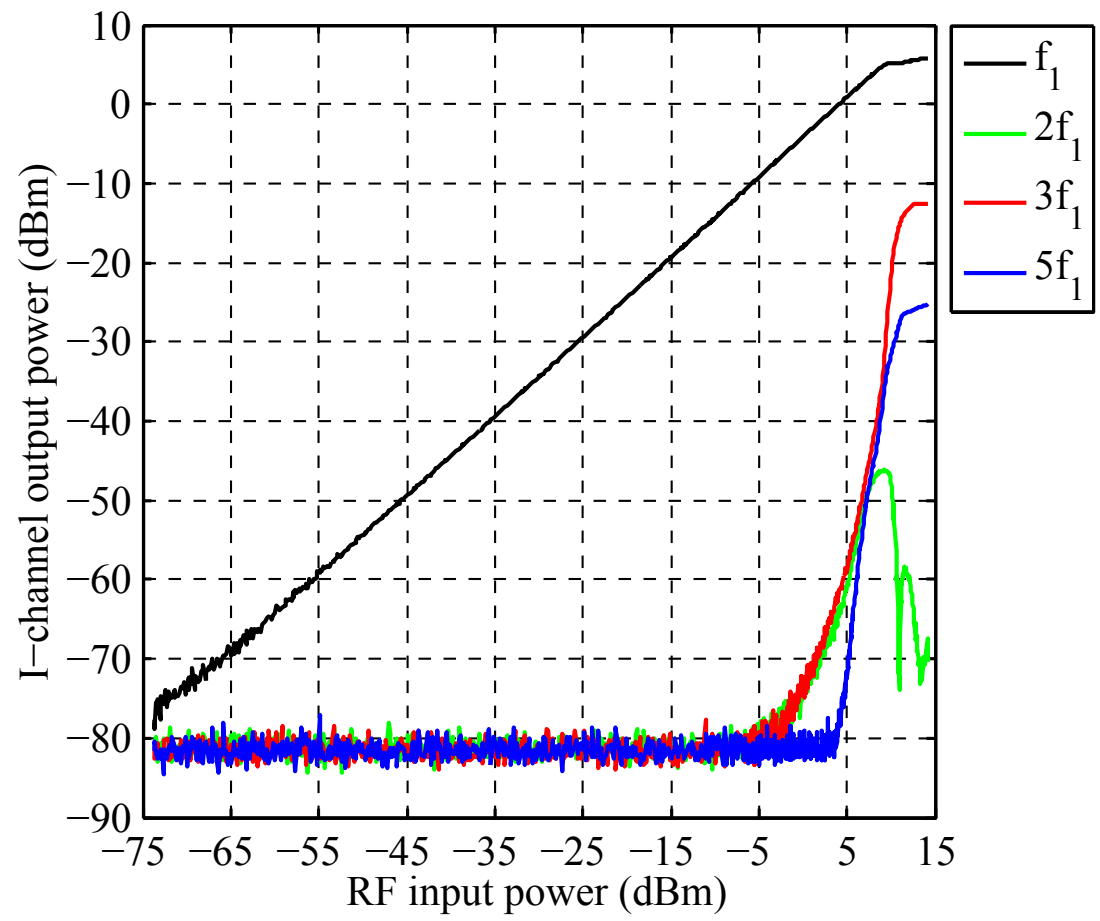

Figure 5.12: The ADL5380 quadrature demodulator's measured I-channel output harmonics versus input power.

The down converted signal at the in-phase channel is measured with a R\&S FSEK30 spectrum analyser. For the measurement the insertion losses of the low-pass filter, coaxial cable, and balun are de-embedded. The results are shown in Fig. 5.12, where the fundamental frequency $f_{1}$ is at $1 \mathrm{MHz}$ and the second, third and fifth harmonics are found at $2 \mathrm{MHz}, 3 \mathrm{MHz}$, and $5 \mathrm{MHz}$ respectively. By analysing the fundamental frequency component, the $1 \mathrm{~dB}$ compression point and linear power gain is determined. The results are shown in Table 5.4.

The strongest and thus most problematic harmonics are depicted in Fig. 5.12. In the 
case of a baseband receiver the harmonics are detected as false targets while it is possible to remove the spurious signals when using an IF receiver. This is assuming that the IF is at least greater than the receiver's baseband bandwidth

$$
f_{I F}>\Delta f
$$

Therefore the harmonics generated by the fundamental signal fall outside the receiver's bandwidth

$$
n f_{1}>f_{I F}+\Delta f, \quad n=2,3, \cdots .
$$

Table 5.4: Measured ADL5380 demodulator parameters with the I and Q output channels differentially loaded by $450 \Omega$

\begin{tabular}{c|c|c}
\hline \hline Parameter description & Symbol & Value \\
\hline Input 1 dB compression point & OP1dB & $10.405 \mathrm{dBm}$ \\
Linear power gain & $G_{\text {demod }}$ & $-4.377 \mathrm{~dB}$ \\
\hline
\end{tabular}

Equation (5.5.2) shows that the IF receiver can recover from single tone harmonic distortions. However, it is not desirable that the demodulator should saturate because large signal excitation degrades the device's noise figure. This is partly due to the reciprocal mixing of the LO's phase noise onto the unwanted blocking signal. Therefore, small target signals, that are spectrally near to the blocker, could be masked.

The demodulator's linear open circuit voltage gain $\left(G_{v, \text { demod }}\right)$ used in the receiver chain noise analysis in Chapter 4 is determined from the linear power gain $\left(G_{\text {demod }}\right)$ by applying transformer theory and voltage division. Figure 5.13 is the circuit equivalent model of the measurement set-up shown in Fig. 5.11.

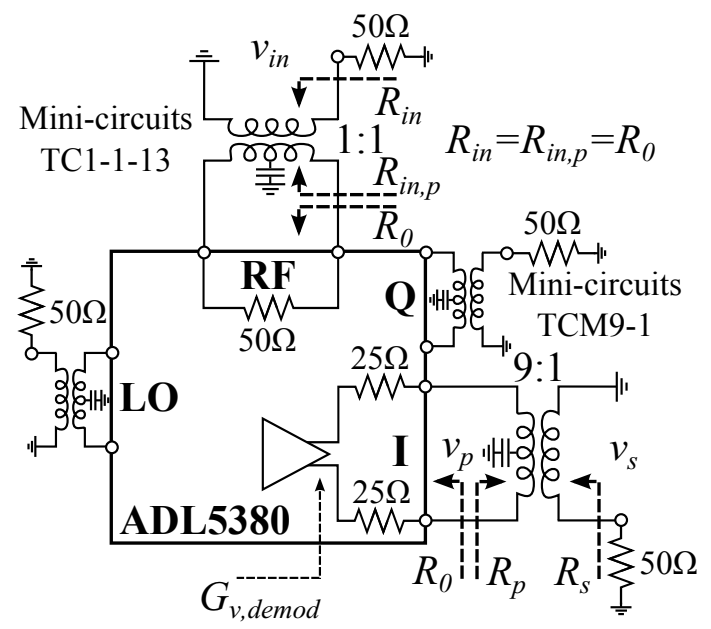

Figure 5.13: The ADL5380 IQ-demodulator measurement set-up circuit model representation used to determine the devices open circuit voltage gain $G_{v, \text { demod }}$.

The Mini-Circuits TCM9-1 9:1 impedance transformer used as a baseband balun has a turn ratio of

$$
n=\frac{N_{p}}{N_{s}}=3,
$$


where $N_{p}$ is the number of turns on the primary side and $N_{s}$ on the secondary. The impedance seen on the primary side of the transform $\left(R_{p}\right)$ when related to the turn ration and impedance on the secondary port $\left(R_{s}\right)$ is calculated using Eq. (5.5.4).

$$
R_{p}=n^{2} R_{s}
$$

The voltage gain from the input to the secondary port of the TCM9-1 transformer $\left(G_{v, s}\right)$ is equal to the measured power gain $\left(G_{\text {demod }}\right)$ because both the input and output impedances are matched, $R_{s}=R_{\text {in }}=R_{0}=50 \Omega$,

$$
\begin{aligned}
G_{\text {demod }} & =10 \log \left(\frac{v_{s}^{2} / R_{s}}{v_{i n}^{2} / R_{i n}}\right), \\
& =20 \log \left(\frac{v_{s}}{v_{i n}}\right), \\
& =G_{v, s}
\end{aligned}
$$

where $v_{i n}$ is the input voltage at the demodulator's RF port. The transformer's insertion loss is already taken into account, thus, ideal transformer behaviour is assumed. The power gain from the input to the primary and secondary ports of the 9:1 transform are equal because $v_{p}=n v_{s}$ and

$$
\begin{aligned}
G_{v, p} & =10 \log \left(\frac{v_{p}^{2} / R_{p}}{v_{i n}^{2} / R_{i n}}\right) \\
& =10 \log \left[\frac{\left(n v_{s}\right)^{2} /\left(n^{2} R_{s}\right)}{v_{i n}^{2} / R_{i} n}\right], \\
& =G_{\text {demod }}
\end{aligned}
$$

The impedance seen on the primary side of the TCM9-1 transformer is $R_{p}=450 \Omega$ and the demodulator's differential I and $\mathrm{Q}$ channel output impedances are $R_{0}=50 \Omega$. The demodulator's open circuit voltage gain is calculated using voltage division,

$$
\begin{aligned}
G_{v, \text { demod }} & =10 \log \left[\frac{\left(v_{p} \frac{450 \Omega+50 \Omega}{450 \Omega}\right)^{2} / R_{p}}{\frac{v_{i n}^{2}}{R_{\text {in }}}}\right], \\
& =G_{\text {demod }}+20 \log \left(\frac{450 \Omega+50 \Omega}{450 \Omega}\right), \\
& =-3.4 \mathrm{~dB} .
\end{aligned}
$$

\subsection{Approximate device non-linearity}

A device's non-linear behaviour can be described by different models that have varying degrees of accuracy and complexity. For the case under consideration two FMCW radar receiver implementations are compared. Therefore it is assumed that the same components with similar specifications and non-linear behaviour are used in both implementations. Thus the radar topologies are under scrutiny and not component performance.

The goal in this section is to produce an algebraic expression that allows easy calculation of any non-linear component generated by multi-tone excitation. This approach 
avoids the need for numerical simulation techniques such as harmonic balance analysis or time domain transient analyses. The time domain analyses can be time consuming because numerous periods of the steady-state output is required to produce high enough spectral resolution. This technique is memory and time intensive if the circuit has large time constants and slow transient behaviour.

There are many challenges in modelling an amplifier but it is dependent on the level of abstraction allowed. An op-amp can be modelled by a complex circuit of many transistors, resistors and capacitors that closely replicate the inner workings of the device. But due to the high complexity, a simplified model that approximates the non-linear behaviour of the op-amp is found (see section 5.6.2).

\subsubsection{Operational amplifier non-linearity}

A non-linear model for the Linear Technology LT6200 op-amp, labelled 3 in Figs. 5.1 and 5.14 , is proposed for the multi-carrier input excitation and the time domain interferometric investigation. Figure 5.14 is a simplified receiver chain flow diagram that illustrates the components at risk of saturating.

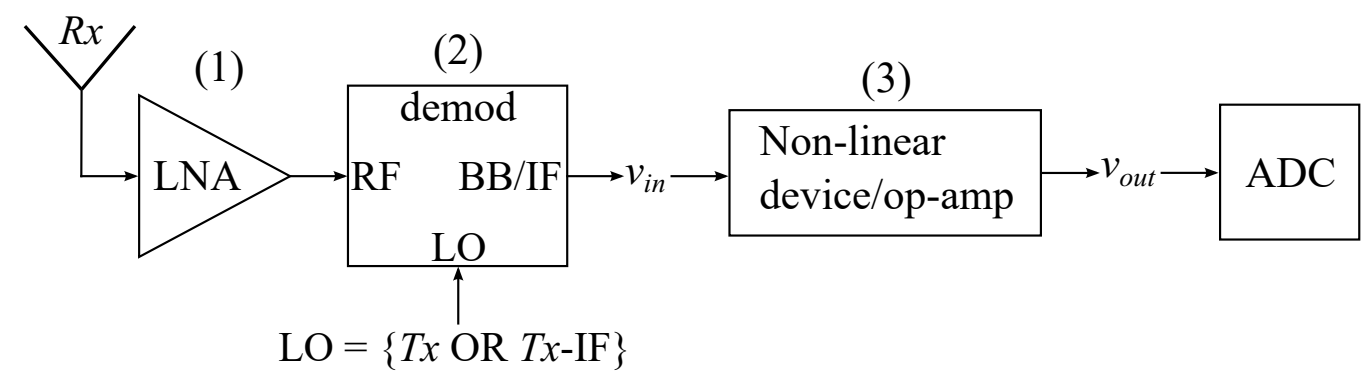

Figure 5.14: Receiver chain flow diagram.

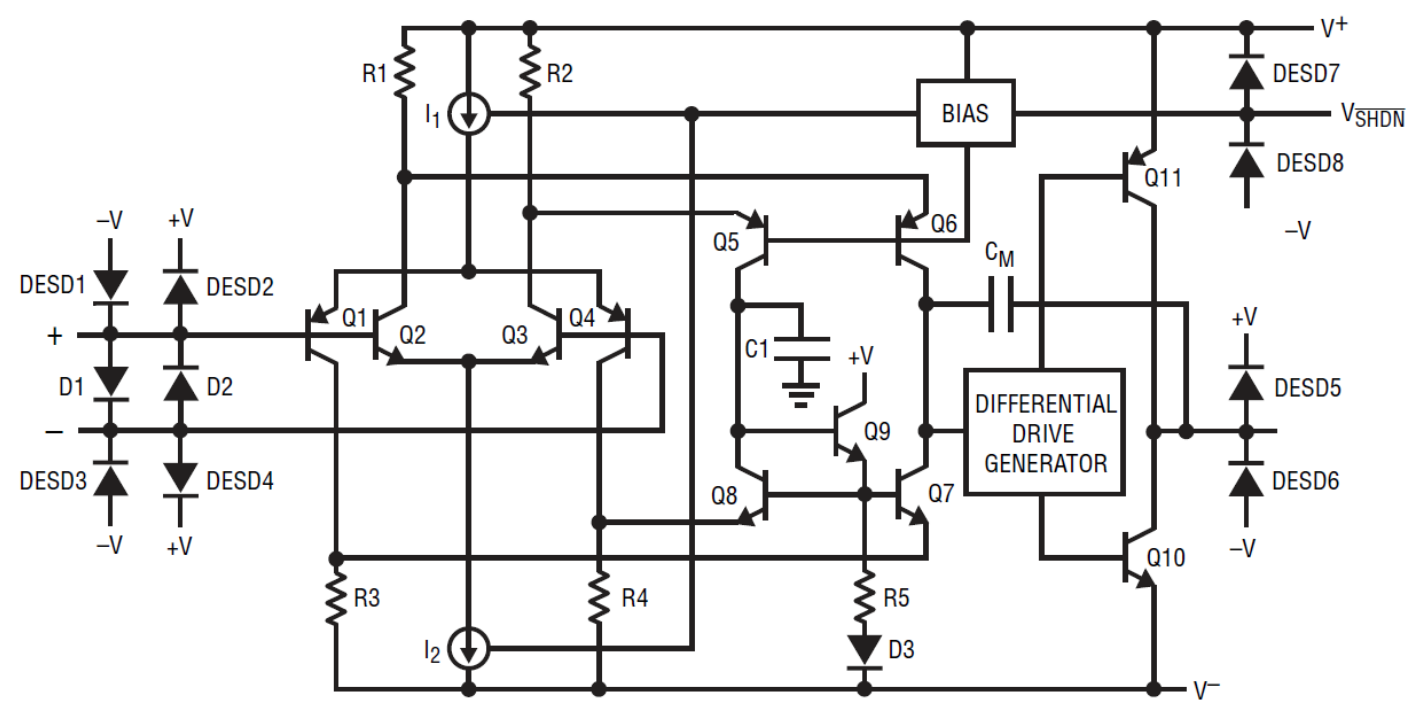

Figure 5.15: Simplified Linear Technology LT6200 schematic [46].

Consider the case where a large initial reflection is received from an object or surface close to the antenna with subsequent smaller reflections coming from objects that are 
further afield. This multi-carrier input signal is described by Eq. (5.6.1), where the signal amplitudes are $A_{1}>A_{2}>\cdots>A_{M}$ and the beat frequencies $\omega_{1}<\omega_{2}<\cdots<\omega_{M}$.

$$
v_{i n}=A_{1} \cos \left(\omega_{1} t\right)+A_{2} \cos \left(\omega_{2} t\right)+\cdots+A_{M} \cos \left(\omega_{M} t\right)
$$

The behaviour of an ideal non-linear op-amp with unity gain is similar to a diode voltage limiter circuit. The voltage limiting circuit consists of two diodes with fixed DCoffset voltages. This model describes the non-linear behaviour of the LT6200 op-amp used in the receiver circuit. The op-amp schematic in Fig. 5.15 shows the op-amp's input and output pins with back-to-back diodes that protect the circuit by limiting the voltage swing.

The diode voltage limiter model derived in section 5.6.2 is programmed in MATLAB using the algorithm described in section 5.6.3. The simulated results are then compared to measurements to empirically verify the model (see section 5.6.4).

\subsubsection{Diode voltage limiter model}

A diode voltage limiter model is proposed to describe the input versus output voltage curve of an op-amp circuit. The input-output behaviour is then approximated by a power series expansion. The algorithm described in section 5.6.3 calculates the amplitude of selected harmonics and IMPs. It allows for high generality with regards to the character and number of input carrier signals.

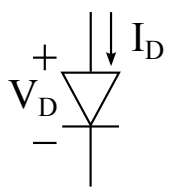

Figure 5.16: Ideal diode representation.

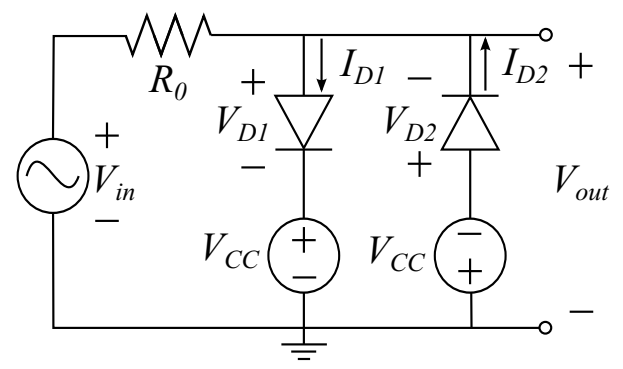

Figure 5.17: Diode voltage limiter circuit.

For the diode circuit analysis it is important to note that Kirchhoff's circuit laws apply to both non-linear and linear circuits [47]. With reference to Fig. 5.16, the diode voltage $V_{D}$ and current $I_{D}$ are related by the ideal diode equation

$$
I_{D}=I_{S}\left[\exp \left(\frac{V_{D}}{n V_{T}}\right)-1\right]
$$

where $I_{S}$ is the diode's reverse-saturation current, $n$ is the emission coefficient or ideality factor, and $V_{T}$ is the thermal voltage,

$$
V_{T}=\frac{k T}{q}
$$


In Eq. (5.6.3) the parameter $k$ is the Boltzmann's constant, $T$ is the diode's absolute temperature, and $q$ is the magnitude of the electron charge.

Eq. (5.6.2) is applied to analyse the diode voltage limiter circuit shown in Fig. 5.17. The output voltage is described as follows

$$
\begin{gathered}
V_{\text {out }}=V_{D 1}+V_{C C}=-V_{D 2}-V_{C C}, \\
V_{\text {out }}=V_{\text {in }}-R_{0}\left[I_{D 1}-I_{D 2}\right],
\end{gathered}
$$

where the input signal is defined by Eq. (5.6.1). The voltages across the diodes are given by

$$
\begin{gathered}
V_{D 1}=V_{\text {out }}-V_{c c}, \\
V_{D 2}=-V_{\text {out }}-V_{c c},
\end{gathered}
$$

and the current through the diodes are

$$
\begin{aligned}
& I_{D 1}=I_{S}\left[\exp \left(\frac{V_{D 1}}{n V_{T}}\right)-1\right], \\
& I_{D 2}=I_{S}\left[\exp \left(\frac{V_{D 2}}{n V_{T}}\right)-1\right] .
\end{aligned}
$$

Substituting Eq. (5.6.6) into (5.6.8) and Eq. (5.6.7) into (5.6.9), and subtracting the latter resultant equation from the former produces Eq. (5.6.10).

$$
I_{D 1}-I_{D 2}=I_{S} \exp \left(-\frac{V_{C C}}{n V_{T}}\right)\left[\exp \left(\frac{V_{\text {out }}}{n V_{T}}\right)-\exp \left(-\frac{V_{\text {out }}}{n V_{T}}\right)\right] .
$$

The trigonometric identity for the hyperbolic sinusoidal equation is identified, reducing Eq. (5.6.10) to

$$
I_{D 1}-I_{D 2}=2 I_{S} \exp \left(-\frac{V_{C C}}{n V_{T}}\right) \sinh \left(\frac{V_{\text {out }}}{n V_{T}}\right) .
$$

Substituting Eq. (5.6.11) into Eq. (5.6.5) results in the output voltage

$$
V_{\text {out }}=V_{\text {in }}-2 R_{0} I_{S} \exp \left(-\frac{V_{C C}}{n V_{T}}\right) \sinh \left(\frac{V_{\text {out }}}{n V_{T}}\right) .
$$

Equation (5.6.12) is transcendental because $V_{\text {out }}$ is contained within a trigonometric function and cannot be solved algebraically in terms of $V_{i n}$. To find a solutions to this kind of equation one can use either a graphical or numerical method. The graphical approach sets each side of a single variable transcendental equation equal to a dependent variable. A solutions is found at the point of intersection when both variables are graphed on the same axes. The numerical solution extends from the graphical method by finding the point at which the intersection occurs using some kind of numerical calculation.

Eq. (5.6.12) can be approximated by truncating the Taylor series. The number of terms in the Taylor series required to approximate a solution is depended on the desired accuracy. However, more terms are needed when the output signal becomes more nonlinear.

A root finding algorithm can be used to identify an approximate answer to Eq. (5.6.12), e.g. Newton's method (also known as the Newton-Raphson method). The MATLAB function called $f$ zero is a root finding algorithm and is used to solve Eq. (5.6.13).

$$
0=V_{\text {in }}-2 R_{0} I_{S} \exp \left(-\frac{V_{C C}}{n V_{T}}\right) \sinh \left(\frac{V_{\text {out }}}{n V_{T}}\right)-V_{\text {out }}
$$


MATLAB's $f$ zero function is based on the Brent's algorithm which uses the bisection, secant and inverse quadratic interpolation methods to realize the approximation [58].

By fitting the simulated results to measurements made in section 5.6.4 the parameters that describe the model's input-to-output voltage curve are determined (see Table 5.5).

\subsubsection{Model implementation}

The op-amp's non-linear behaviour is computed by a piecewise polynomial approximation of the voltage input versus output curve given by Eq. (5.6.13). As in Eq. (5.3.3) the non-linear device's output is expressed as a power series expansion of the input. The input signal is a sum of cosine waves with different frequencies and amplitudes.

Determining the complete expansion of the non-linear output signal is problematic. But it is possible to calculate the amplitudes of specific harmonics or IMPs of interest. Given that the input to a non-linear two-port device is excited by an arbitrary sum of cosine waves, where the device's input-to-output characteristics are described by a power series. An algorithm derived by Sea and Vacroux in [59] is used to determine the amplitude of any frequency component at the device's output port.

A mathematical expansion provided in Appendix A.2 is used to implement a piecewise application of the algebraic formulation described in [59] and [60]. The implementation is scripted in MATLAB and the code is provided in Appendix B.6.

The input versus output voltage curve that describes the model defined in section 5.6.2 is approximated by a truncated Maclaurin series. The Maclaurin series coefficients are used to solve for a specific frequency component by assigning values to the $\alpha$ 's that indicate the IMP or harmonic of interest. The order of the selected component is defined by Eq. (A.2.14). Equations (A.2.20) and (A.2.21) are applied to solve Eq. (A.2.17) producing the desired frequency component's amplitude.

\subsubsection{Operational amplifier test set-up}

The parameters required for the Linear Technology LT6200 op-amp's non-linear model (discussed in section 5.6.2) are determined from measurements. For the measurements the op-amp is set-up in a summing configuration as shown in Fig. 5.18. The op-amp has a voltage gain of $15 \mathrm{~dB}$ to ensure sufficient saturation of its output. The load impedance consists of the spectrum analyser's $50 \Omega$ input impedance and a $330 \Omega$ resistor. The 330 $\Omega$ resistor is added to reduce the op-amp's output current. The circuit's output spectrum is measured using the R\&S FSEK30 spectrum analyser. By applying voltage division the signal amplitude at the op-amp's output is determined.

As discussed in section 5.5 the signal generator produces spurious harmonics. These harmonics must be below $-70 \mathrm{dBm}$ to allow the op-amp harmonics to dominate at low saturation levels.

By measuring the signal generator's output the required harmonic rejection is determined. At a carrier frequency of $0.95 \mathrm{MHz}$ the second and third harmonics are $-50 \mathrm{dBc}$ and $-69 \mathrm{dBc}$ respectively. Therefore, when the carrier's output power level is $0 \mathrm{dBm}$, a fifth-order Chebyshev $3 \mathrm{~dB}$ equal-ripple low-pass filter (LPF) will provide sufficient attenuation. The low pass filter prototype's lump element values are documented in [8].

The filter is matched to $50 \Omega$ and has a cut-off frequency of $1.082 \mathrm{MHz}$, its ladder network is shown in Fig. 5.19. In reality the filter's load impedance is the parallel combination of resistors $R_{L}(51 \Omega)$ and $R_{G 1}(1 \mathrm{k} \Omega)$ producing a value close to $50 \Omega$. As 
presented in Fig. 5.20 the filter has more than $40 \mathrm{~dB}$ attenuation at the signal generator's second harmonic $(1.9 \mathrm{MHz})$.

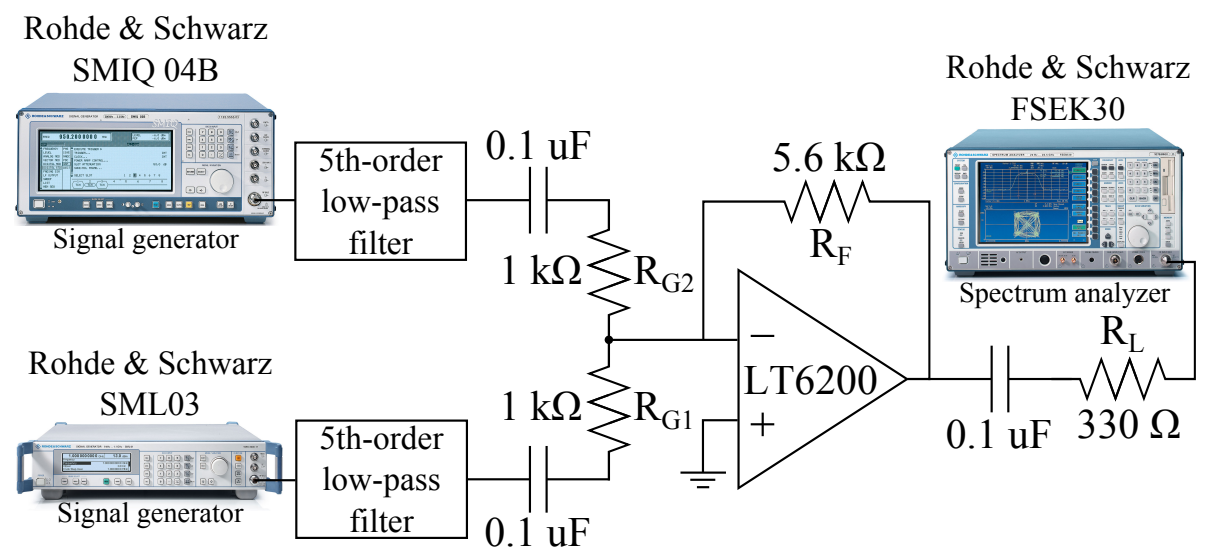

Figure 5.18: Linear Technologies LT6200 op-amp harmonic and intermodulation measurement setup.

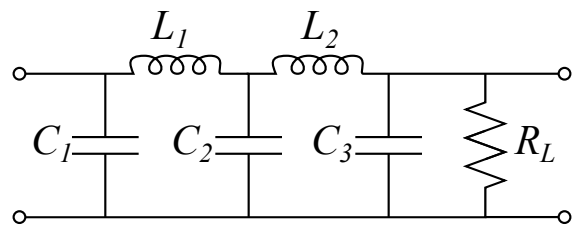

Figure 5.19: Fifth-order low-pass filter ladder network.

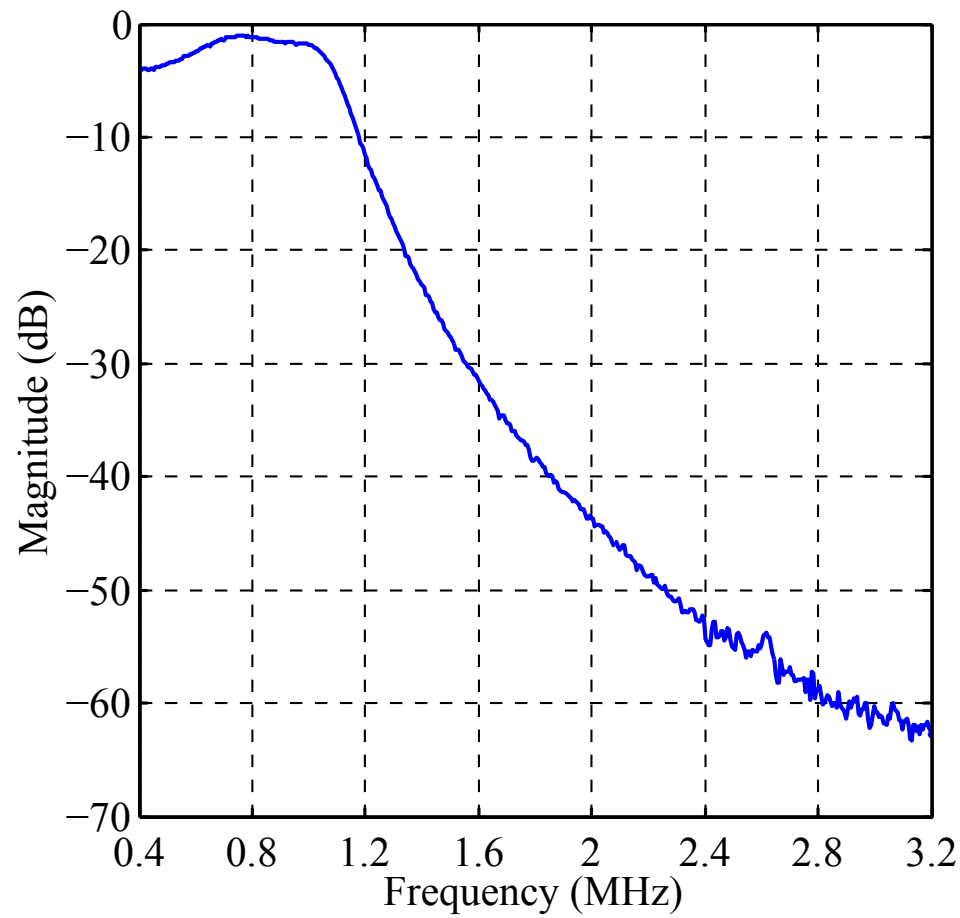

Figure 5.20: Fifth-order 3 dB equal-ripple low-pass filter amplitude response. 


\section{Operational amplifier measurement results and model parameters}

Using the measurement set-up presented in Fig. 5.18 the third order harmonic is measured at different two tone input excitations. For each measurement the first signal generator's output power $\left(P_{1}\right)$ is fixed, while the second signal generator's power $\left(P_{2}\right)$ is increased until both outputs are equal, $P_{2} \rightarrow P_{1}$. The ratio between the fixed and increasing input signals is known as the input amplitude ratio $\left(A_{2} / A_{1}\right)$. The op-amp model parameters, presented in Table 5.5, are determined by minimising the error between the measured and simulated third order harmonic $\left(f_{H 3}=3 f_{1}\right)$ as presented in Fig. 5.21. Due to the spectrum analyser's noise floor there is a large error at low input power levels [e.g. at $\left(A_{2} / A_{1}\right)_{d B}<-40 \mathrm{~dB}$ for $P_{1}=3.3 \mathrm{dBm}$ in Fig 5.21].

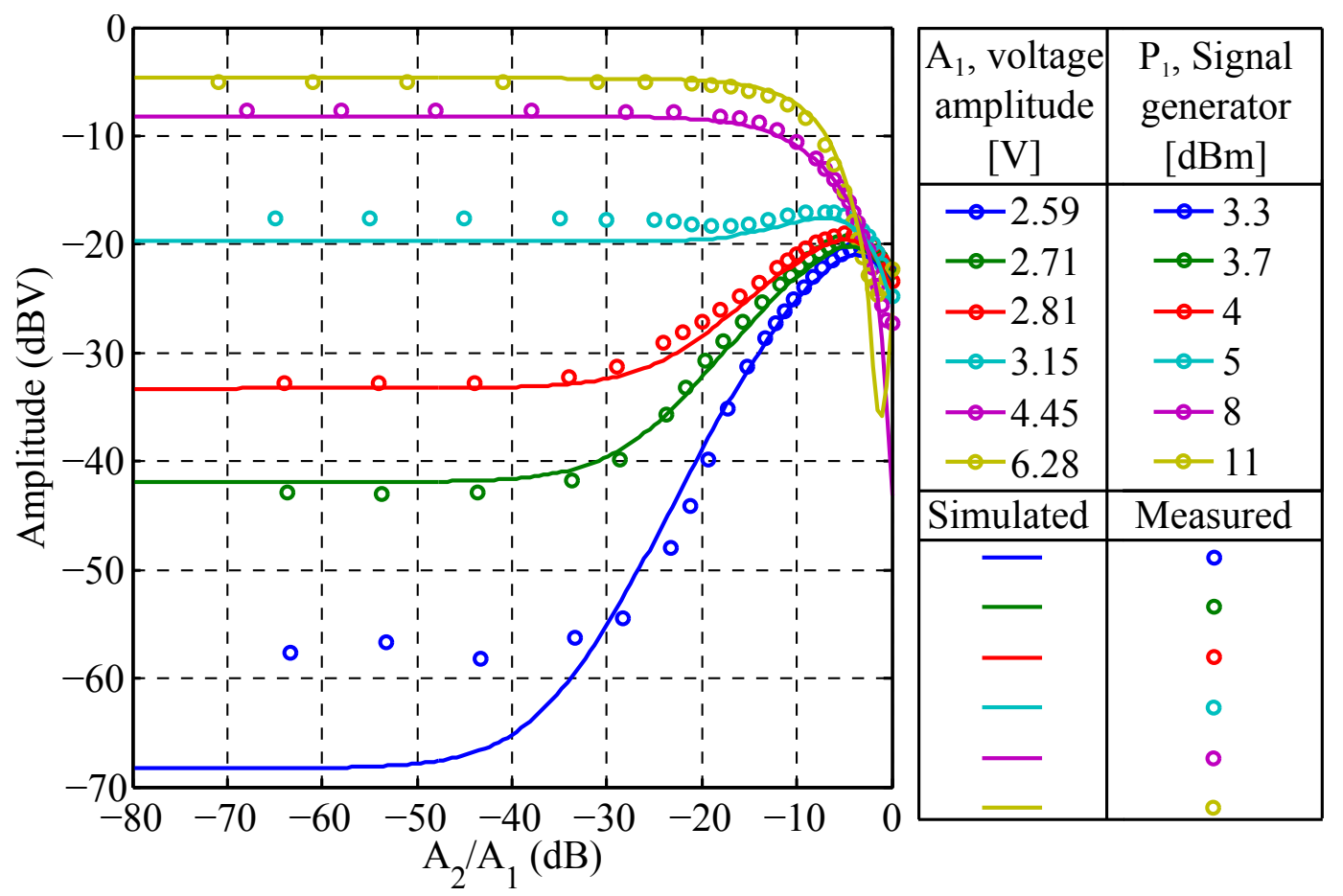

Figure 5.21: Measured and simulated 3rd order harmonic distortion $\left(f_{H 3}=3 f_{1}\right)$ versus the input amplitude ratio.

Figure 5.22 illustrates the spectral location of the most prominent IMPs and harmonics when $P_{1}=P_{2}=3.7 \mathrm{dBm}$. The 3 rd order IMPs at $f_{I M P 3, A}=2 f_{1}-f_{2}$ and $f_{I M P 3, B}=$ $2 f_{2}-f_{1}$ [shown in Fig. 5.22 (a)] are measured and compared to the simulated results in Figs. 5.23 and 5.24 respectively. The level of agreement held between the simulated and measured results allow the non-linear behaviour of the zero-IF and IF receiver topologies to be compared using the proposed model. It is assumed that both receiver topologies are constructed using the same components.

The model describes symmetrical signal distortion, therefore, only odd order harmonics and IMPs are generated by the simulation as seen in Fig. 5.22 (b). In reality both even and uneven order distortions are generated. The 3rd order distortions are the most problematic and are detected as false targets before the 2 nd order components. Therefore the simulation is useful in assessing the worst case scenarios. 


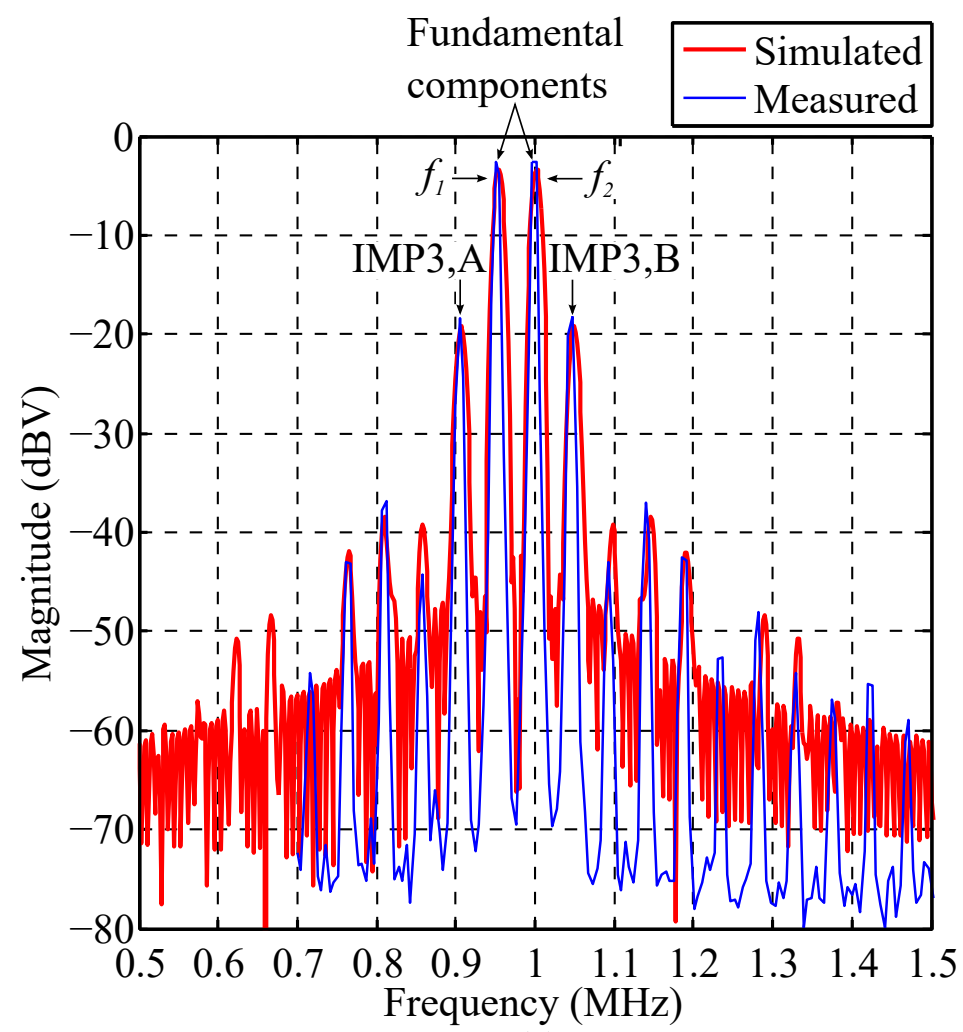

(a)

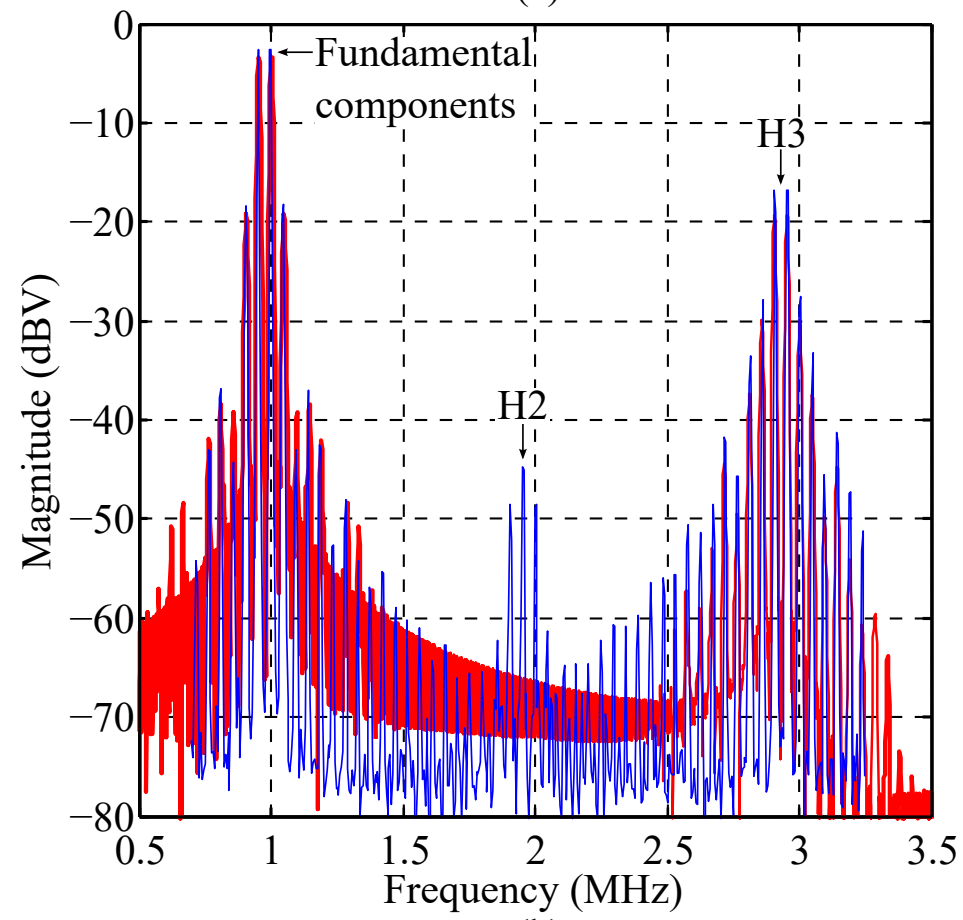

(b)

Figure 5.22: Measured and simulated spectrum when $P_{1}=P_{2}=3.7 \mathrm{dBm}$. 

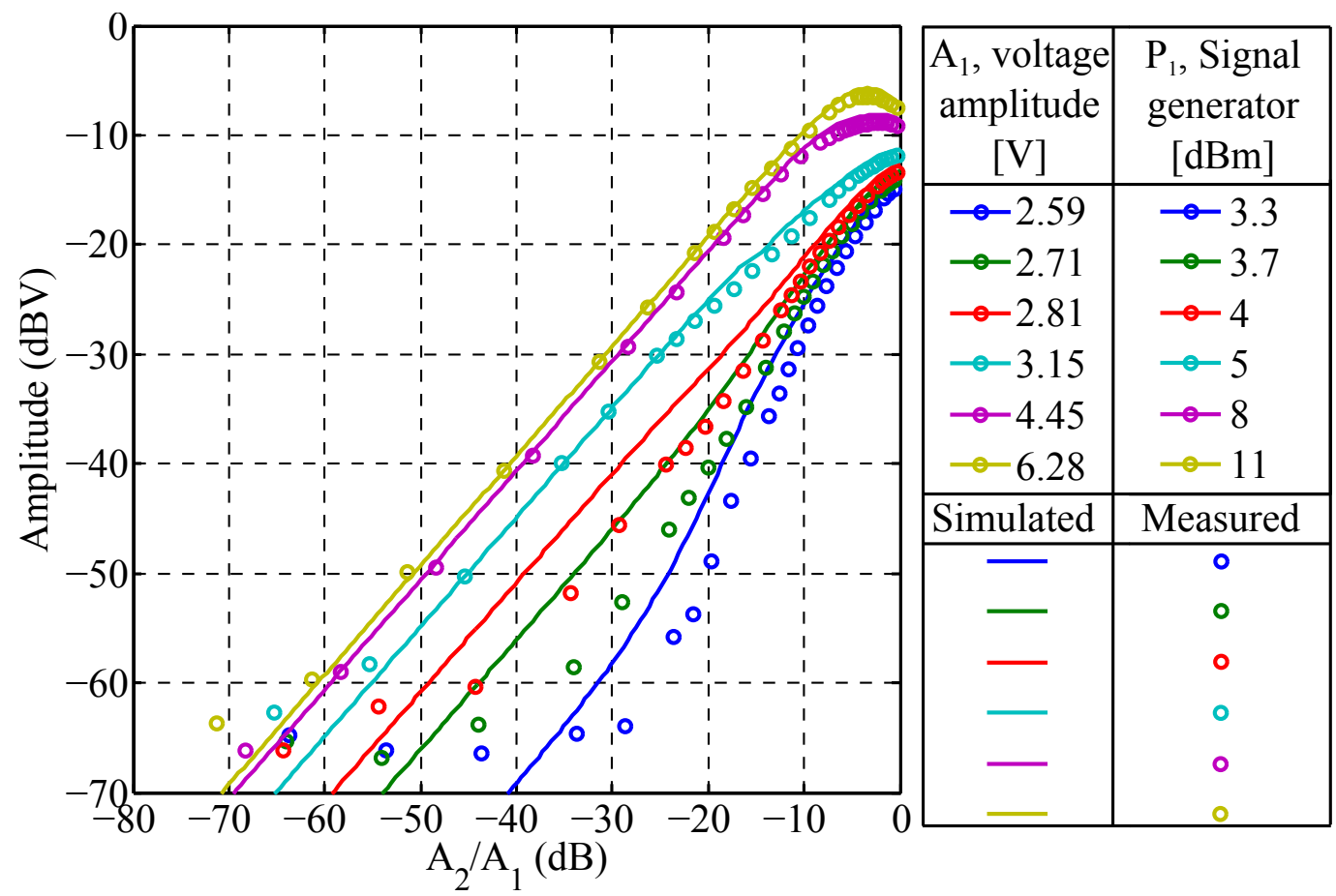

Figure 5.23: Measured and simulated IMP3,A $\left(f_{I M P 3, A}=2 f_{1}-f_{2}\right)$ versus the input amplitude ratio.
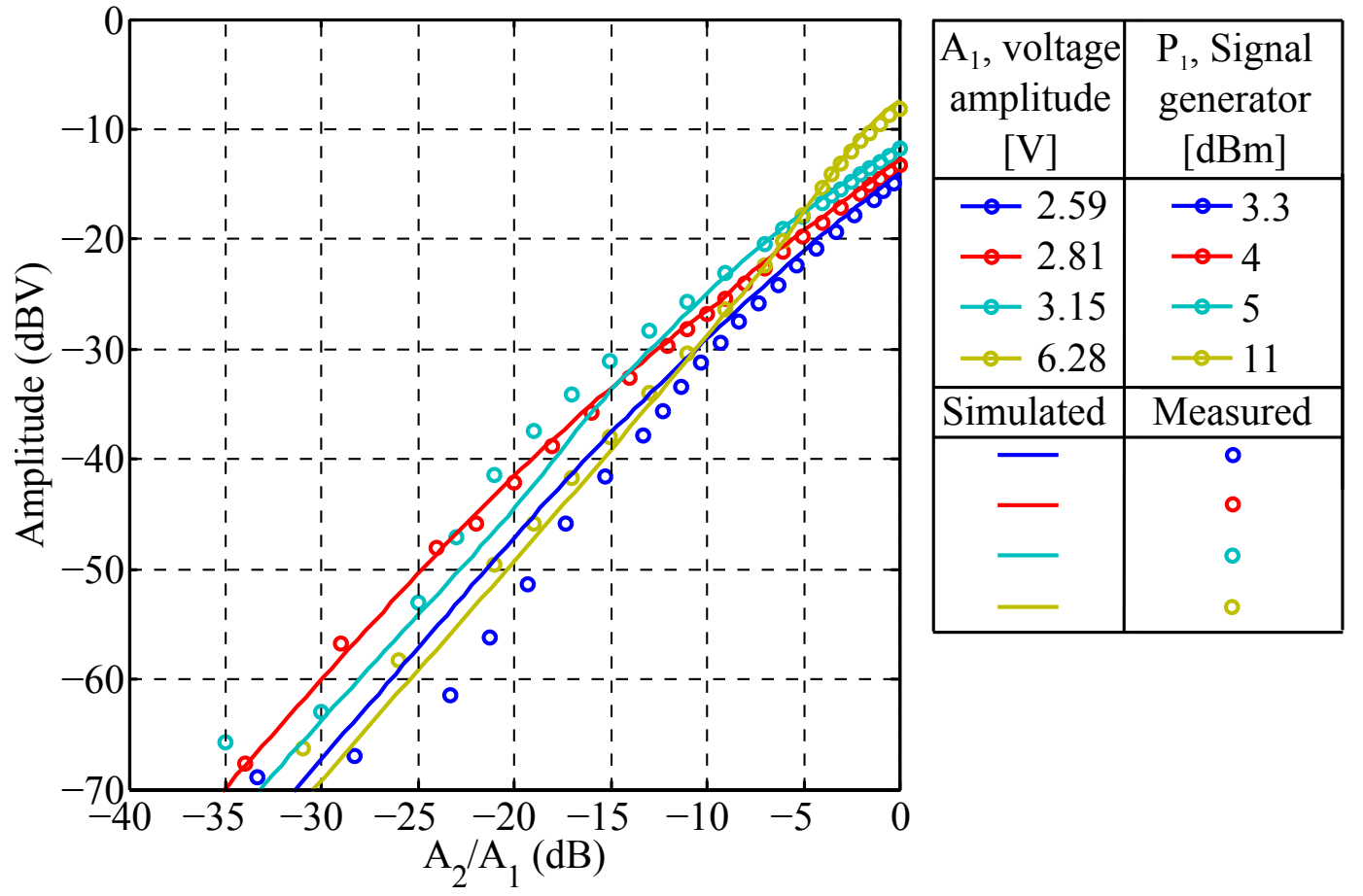

Figure 5.24: Measured and simulated IMP3,B $\left(f_{I M P 3, B}=2 f_{2}-f_{1}\right)$ versus the input amplitude ratio. 
Substituting the parameters in Table 5.5 into Eq. (5.6.13) produces the non-linear model's input-to-output voltage curve presented in Fig. 5.25.

Table 5.5: LT6200 op-amp model parameters

\begin{tabular}{c|c}
\hline \hline Parameter & Value \\
\hline$I_{S}$ & $1 \mathrm{e}-10$ \\
$V_{C C}$ & 2.18 \\
$V_{T}$ & 0.026 \\
$n$ & 1.15 \\
$R_{0}$ & 50 \\
$R_{2} / R_{1}$ & 0.66 \\
\hline
\end{tabular}

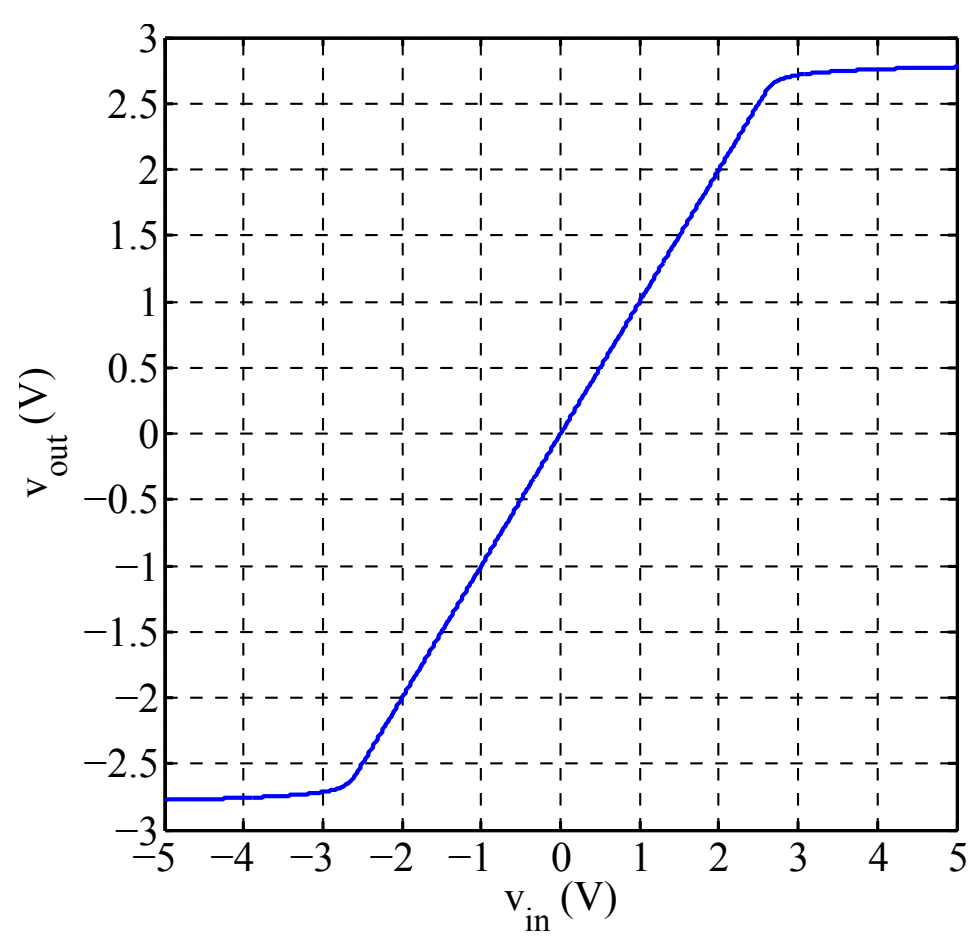

Figure 5.25: The non-linear model's input-to-output voltage curve.

\subsubsection{Non-linear model analysis}

In this section the results from the diode voltage limiter non-linear model are presented. The intention is to analyse the impact multiple carrier's have on the IF receiver when it presents non-linear behaviour.

For specific sets of input signal amplitudes it is possible that the voltage level of intermodulation distortions (IMDs) are lower than that of the harmonic distortions (HDs). It is assumed that the $\operatorname{IF}\left(f_{I F} \approx 1 \mathrm{MHz}\right)$ is at least an order of magnitude greater than the receiver's baseband bandwidth $(\Delta f=100 \mathrm{kHz})$. Therefore, as shown by Eq. (5.6.14), if the second order harmonic $\left(f_{H_{2}}\right)$ is generated by a signal at zero beat frequency. All the 


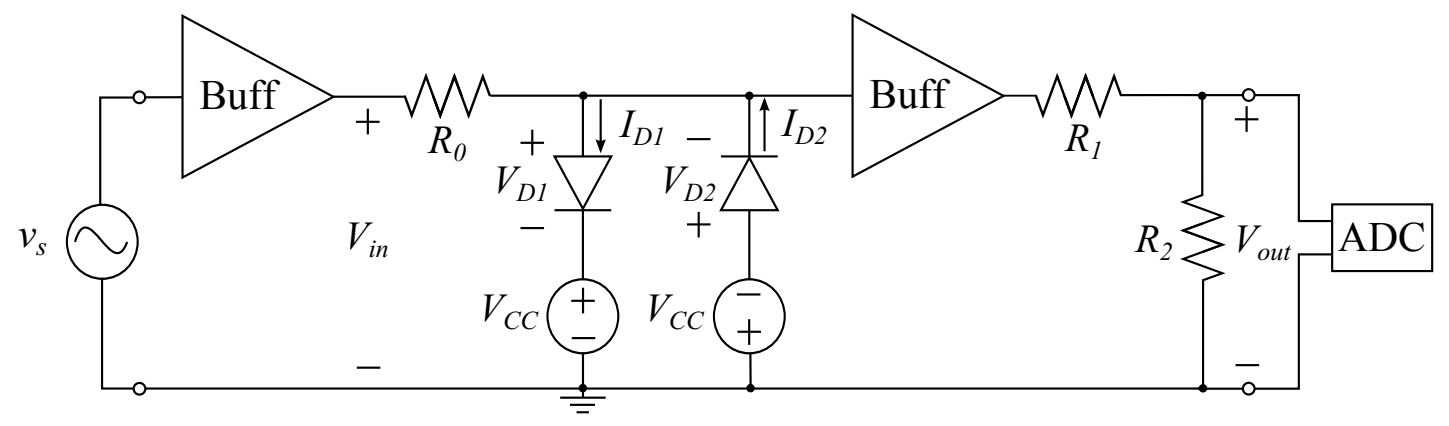

Figure 5.26: Simulated diode voltage limiter circuit.

harmonics are located outside the receiver's bandwidth, subsequently, they are suppressed by the anti-aliasing filter.

$$
f_{H n}>\cdots>f_{H 3}>f_{H 2}=2 f_{I F}>f_{I F}+\Delta f
$$

However, some IMPs fall in the receiver's passband and could be detected as false targets. For specific multi-tone excitations the IF receiver is capable of removing any detectable spurious signals. A spurious signal is assumed to be detectable if its power level is above the MDS level.

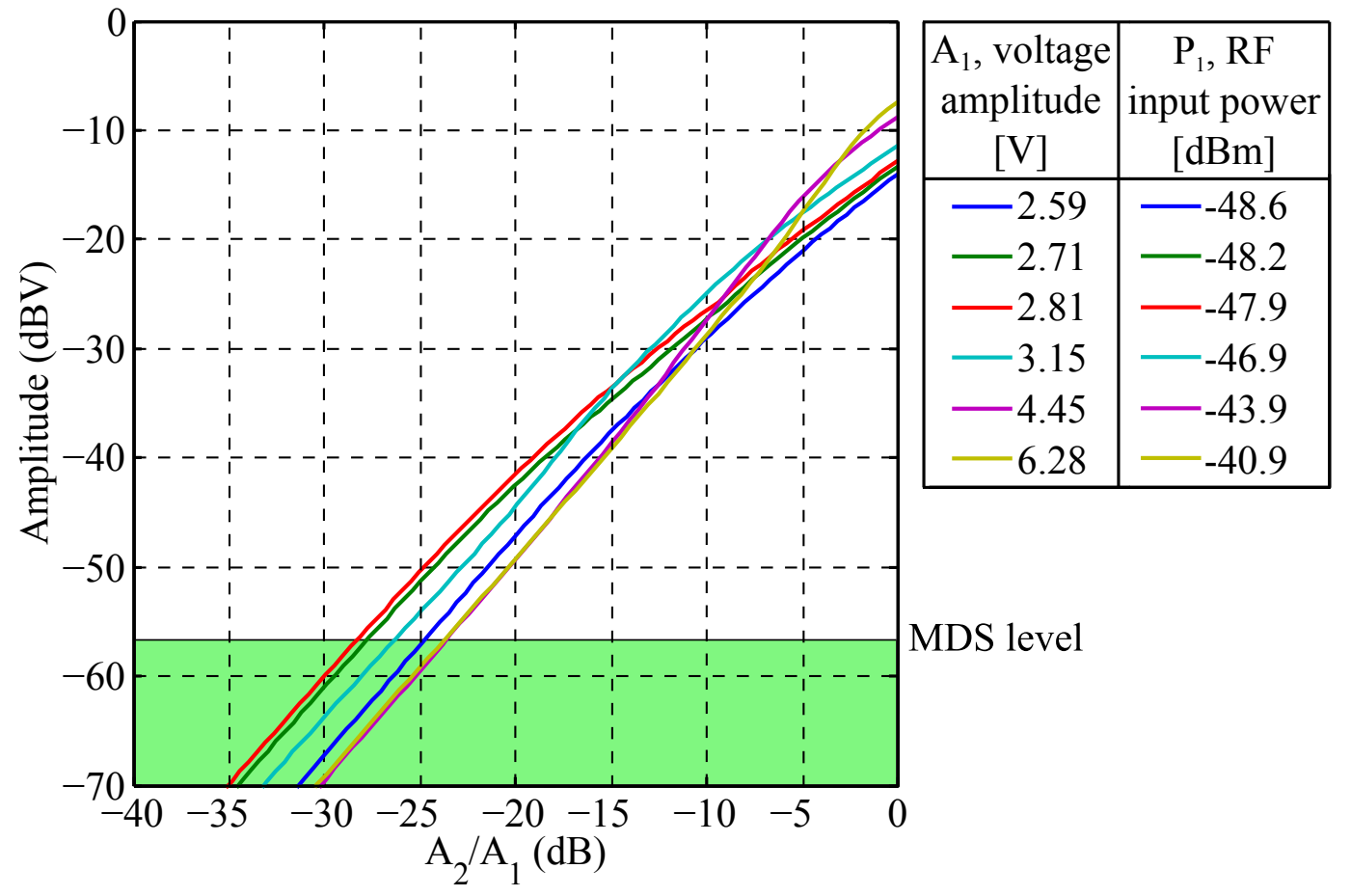

Figure 5.27: Third-order intermodulation product's voltage level at the ADC's input $\left(f_{I M P 3, B}=2 f_{2}-f_{1}\right)$ versus the input amplitude ratio of the fundamental components.

An IF receiver has a greater SFDR compared to an equivalent baseband receiver if it is allowed to operate close to or above its compression point without detecting distortions. The IF receiver's ability to suppress distortions is leveraged to remove false targets.

Figure 5.26 depicts the circuit solved by the algorithm described in section 5.6.3, the results are shown in Figs. 5.27 to 5.29. Voltage division is applied to adjust the $5 \mathrm{~V}$ opamp rail-to-rail voltage to the $3.3 \mathrm{~V}$ input reference to the ADC. The voltage adjustment 
fits the simulated results to the Atmel ATSAM3X8E microprocessor's full-scale ADC input voltage.

As a point of comparison the third order harmonics and IMPs are considered as they are usually strong spurious components that are spectrally near to the fundamental signals. The IF receiver is designed to select the upper sideband. Therefore any signals below the IF are considered to be outside the receiver's bandwidth.

The fundamental signals at frequencies $f_{1}$ and $f_{2}$ are the first and second reflection's beat frequencies, therefore $f_{1}<f_{2}$. It is assumed that the first reflection's amplitude $\left(A_{1}\right)$ is great enough to cause the receiver to saturate and that the third order IMPs (at $f_{I M P 3, A}=2 f_{1}-f_{2}$ and $\left.f_{I M P 3, B}=2 f_{2}-f_{1}\right)$ are located within the receiver's detection bandwidth

$$
f_{I F}<f_{I M P 3, A}<f_{I M P 3, B}<f_{I F}+\Delta f .
$$

For the simulation the first target's amplitude $\left(A_{1}\right)$ is fixed at a value that saturates the op-amp. The second reflection's amplitude $\left(A_{2}\right)$ is increased until $A_{1}=A_{2}$ i.e. the amplitude ratio $\left(A_{2} / A_{1}\right)_{d B}=0 \mathrm{~dB}$. Spurious signals with magnitudes above the MDS level are seen as false targets. The green areas in Figs. 5.27, 5.28 and 5.29 indicate voltage levels at the ADC's input that are below the MDS level.

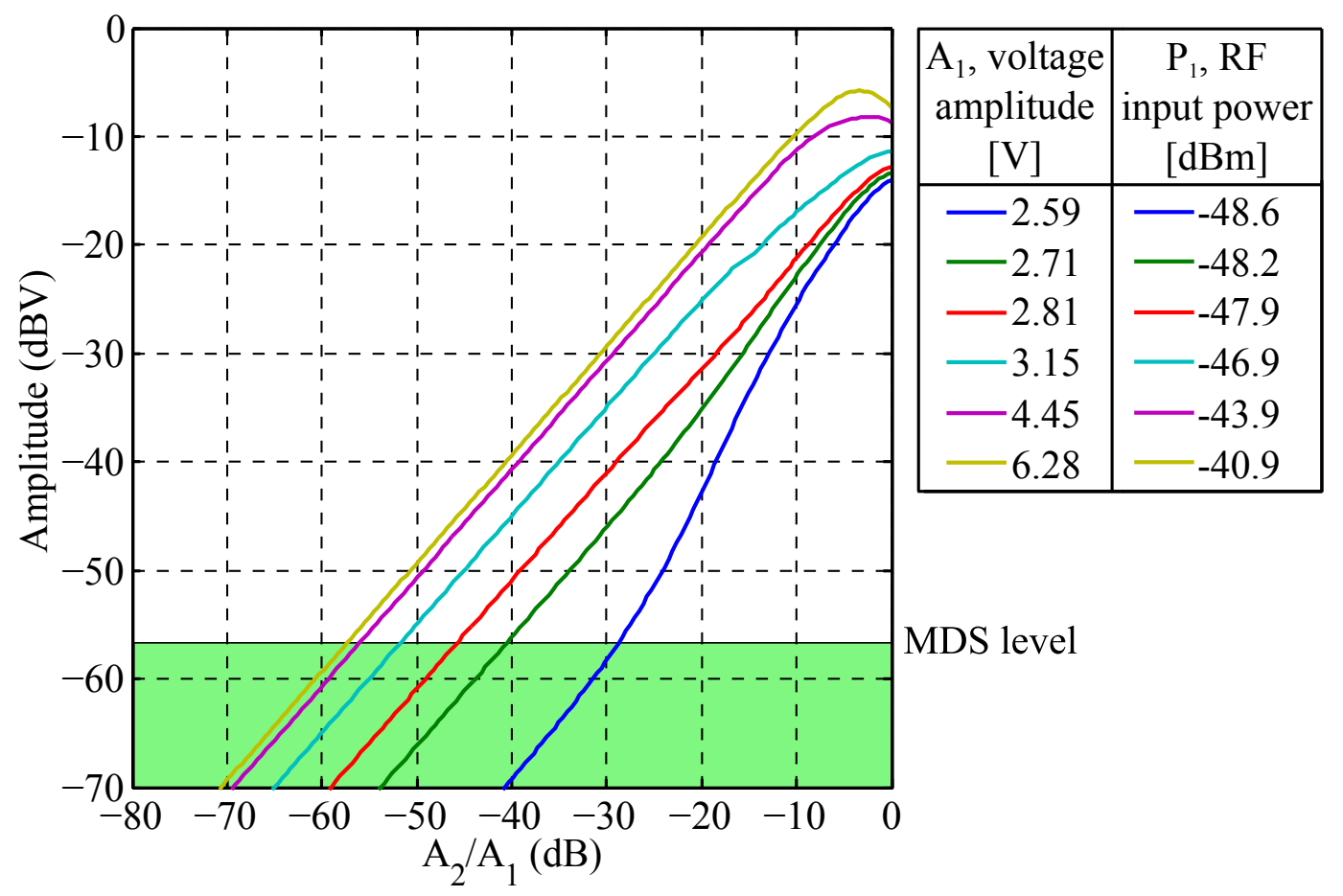

Figure 5.28: Third-order intermodulation product's voltage level at the ADC's input $\left(f_{I M P 3, A}=2 f_{1}-f_{2}\right)$ versus the input amplitude ratio of the fundamental components.

By comparing Figs. 5.27 and 5.28 it is clear that for the same $\left(A_{2} / A_{1}\right)_{d B}$ ratio, the IMP at $f_{I M P 3, A}$ has a greater magnitude than the component at $f_{I M P 3, B}$. Illustrating that IMP3,A is a stronger function of the first fundamental signal than IMP3,B. The magnitude of both IMP's are equal at $\left(A_{2} / A_{1}\right)_{d B}=0 \mathrm{~dB}$. This result corroborates the conclusion made in section 5.3.2.

To assess the worst case scenario, the IMP at $f_{I M P 3, A}$ is considered because it is a strong function of the dominant fundamental component and falls within the receiver's passband. Figure 5.30 illustrates the amplitude ratio between the third order harmonic 
at $3 f_{1}$ (Fig. 5.29) and IMP3,A (Fig. 5.28). For values above $0 \mathrm{~dB}$ in Fig. 5.30 the third order harmonic is greater than the IMP.

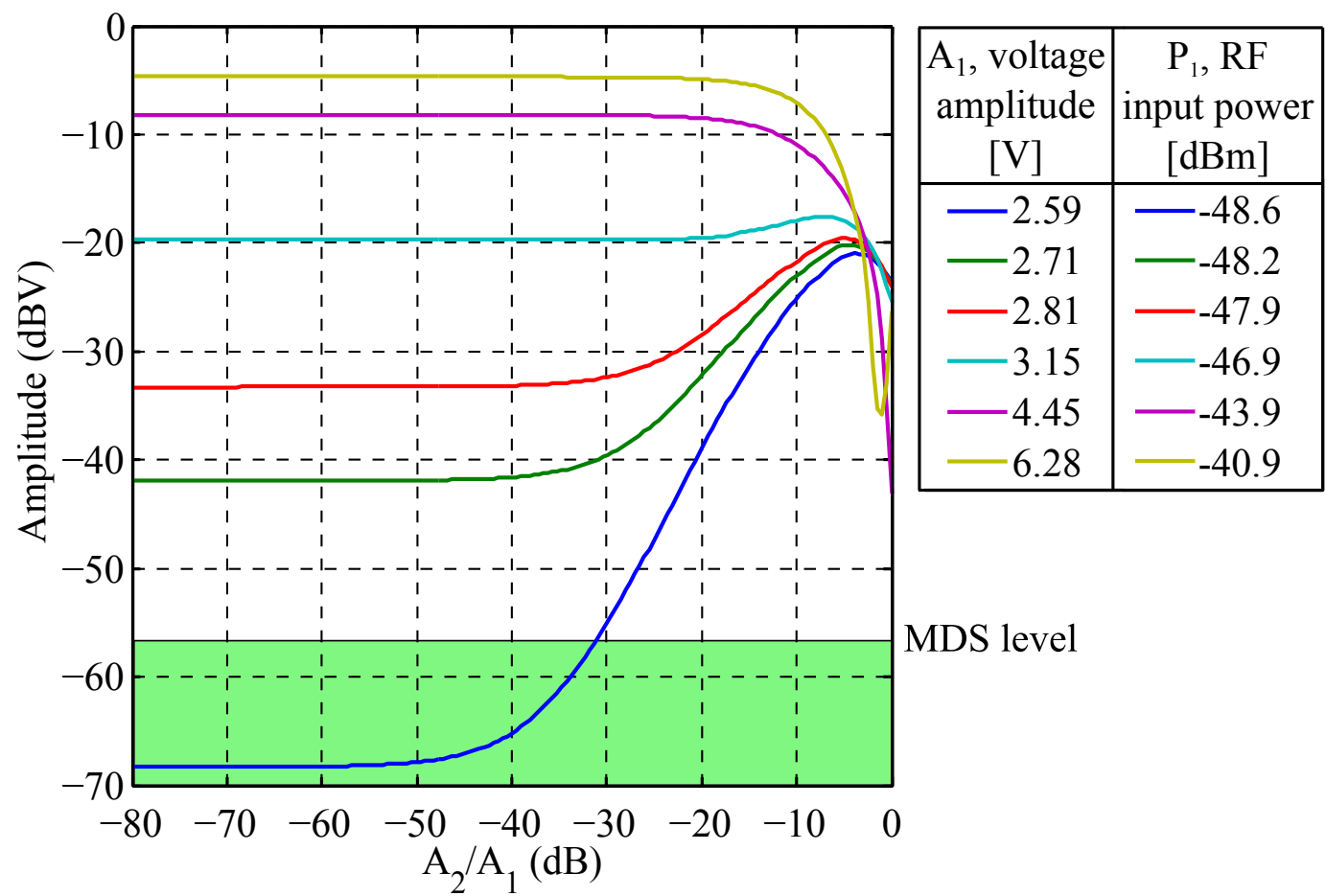

Figure 5.29: Third-order harmonic's voltage level at the ADC's input $\left(f_{H 3}=3 f_{1}\right)$ versus the input amplitude ratio of the fundamental components.

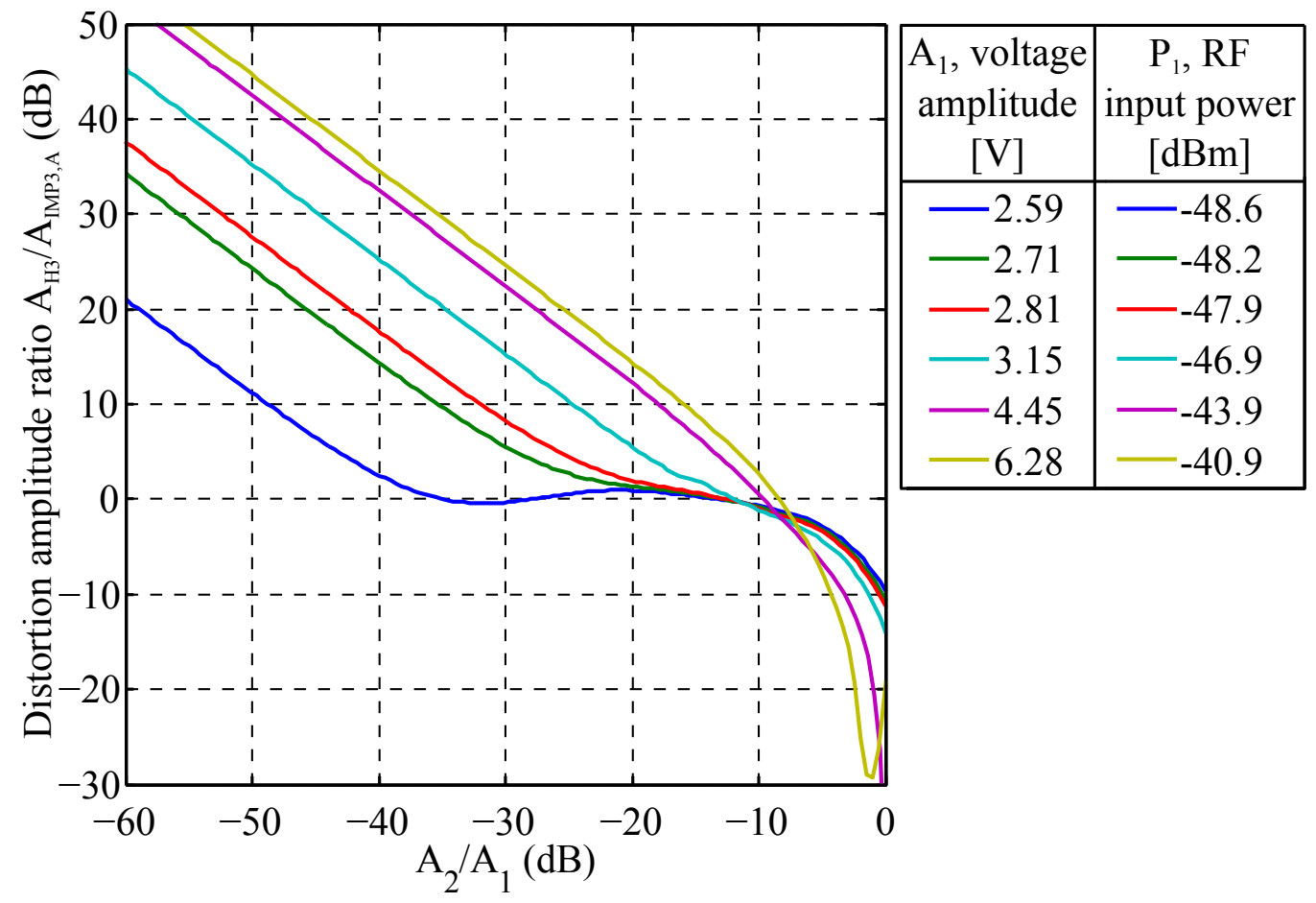

Figure 5.30: Amplitude ratio between the third order harmonic $\left(f_{H 3}=3 f_{1}\right)$ and third order IMP $\left(f_{I M P 3, A}=2 f_{1}-f_{2}\right)$ versus the input amplitude ratio of the fundamental components. 
If the IMP in Fig. 5.28 is below the MDS level while the third order harmonic is greater than both the IMP and MDS level. The IF receiver is capable of removing distortions the baseband receiver can not. In this scenario the IF receiver has a greater SFDR compared to an equivalent zero-IF receiver.

As an example, the first carrier's RF input power is set to $-48.2 \mathrm{dBm}$. The IF filter's $\mathrm{SFC}$ is not taken into account and a flat filter response is assumed.

In Figs. 5.28 and 5.32 it is seen that (for $P_{1}=-48.2 \mathrm{dBm}$ ) the IMP is below the MDS level given that the second fundamental component's magnitude $A_{2}$ is $40 \mathrm{~dB}$ below $A_{1}$. The harmonics and IMPs outside the band of interest, illustrated in Fig. 5.31, are removed by the anti-aliasing filter and no spurious signals are detected by the IF receiver.

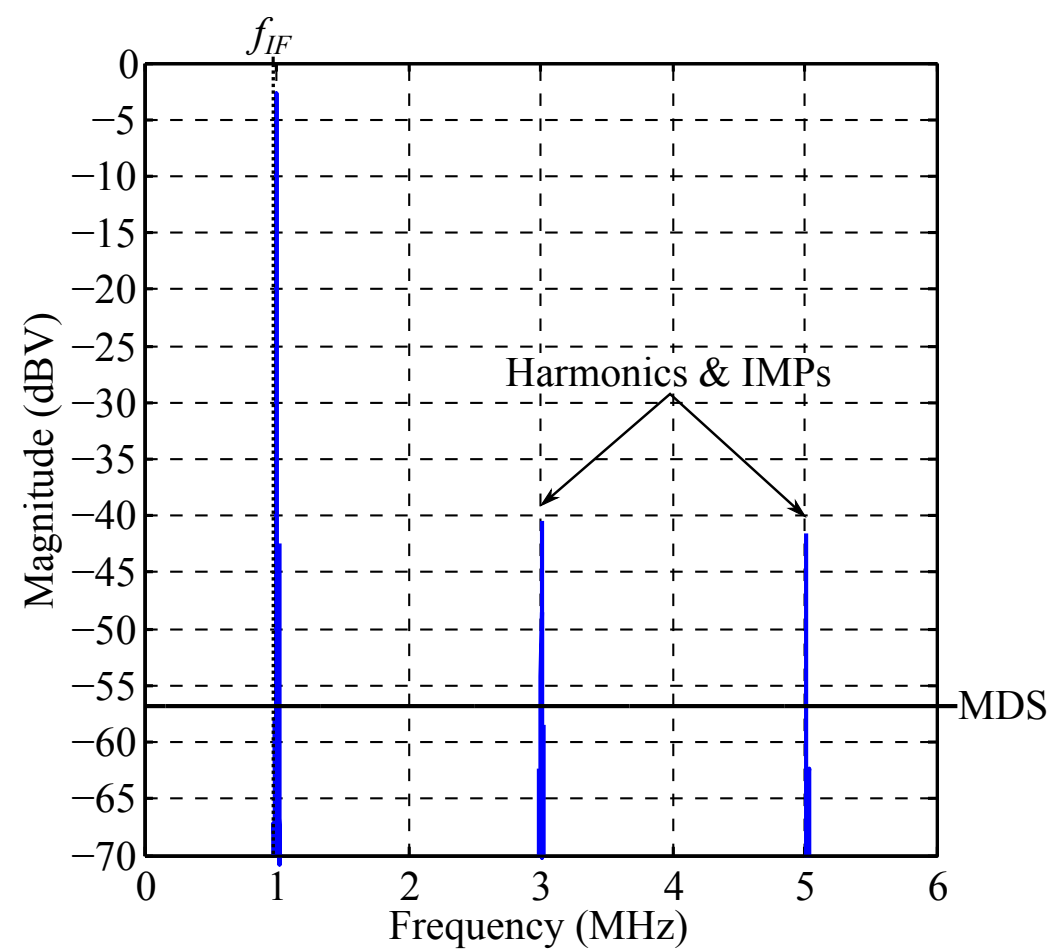

Figure 5.31: IF receiver spectrum, where the harmonics and IMPs fall outside the band of interest.

The distortion amplitude ratio $\left(A_{H 3} / A_{I M P 3, A}\right)_{d B}$ illustrated in Fig. 5.30 is above $0 \mathrm{~dB}$ at input ratios below $-12 \mathrm{~dB}$. Therefore the third harmonic is greater than the IMP when $\left(A_{2} / A_{1}\right)_{d B}<-12 \mathrm{~dB}$. The IF receiver's down converted spectrum is presents in Fig. 5.32. As shown in Fig. 5.33, by applying the same excitation to a baseband receiver results in the detection of the third order harmonic as a false target.

The full scale RF input power $\left(F S_{R X}\right)$ calculated in section 5.2 is $-51.9 \mathrm{dBm}$. An input signal of $-48.2 \mathrm{dBm}$ is received without the detection of false targets. Therefore, in the example, the IF receiver's ability to receive target signals without detecting distortions is $3.7 \mathrm{~dB}$ greater than an equivalent baseband receiver.

The IF receiver is effective at removing harmonic distortions. However, it is still susceptible to low order IMPs that are spectrally near to the fundamental signals. The magnitude and spectral location of IMPs are entirely dependent on the received signals causing the distortion. 


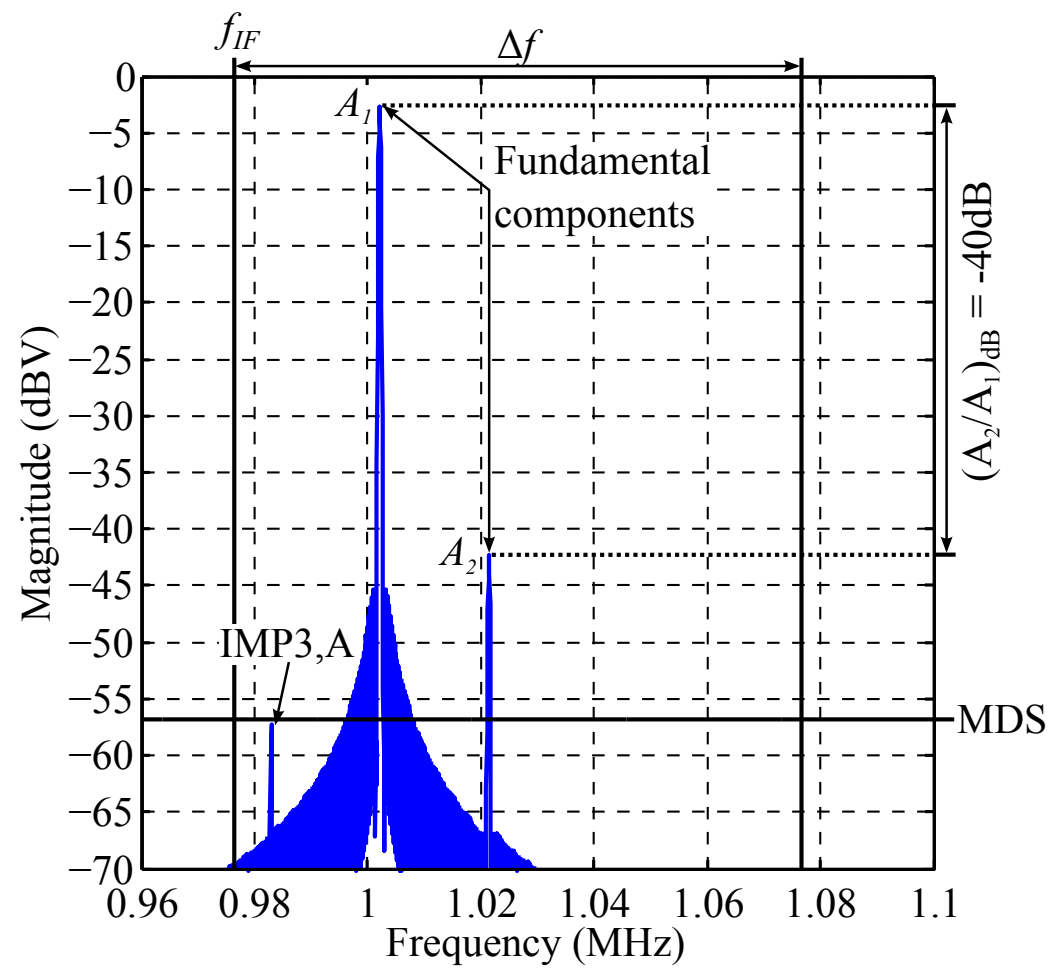

Figure 5.32: IF receiver spectrum, where the fundamental components are detected and the IMP is below the MDS level.

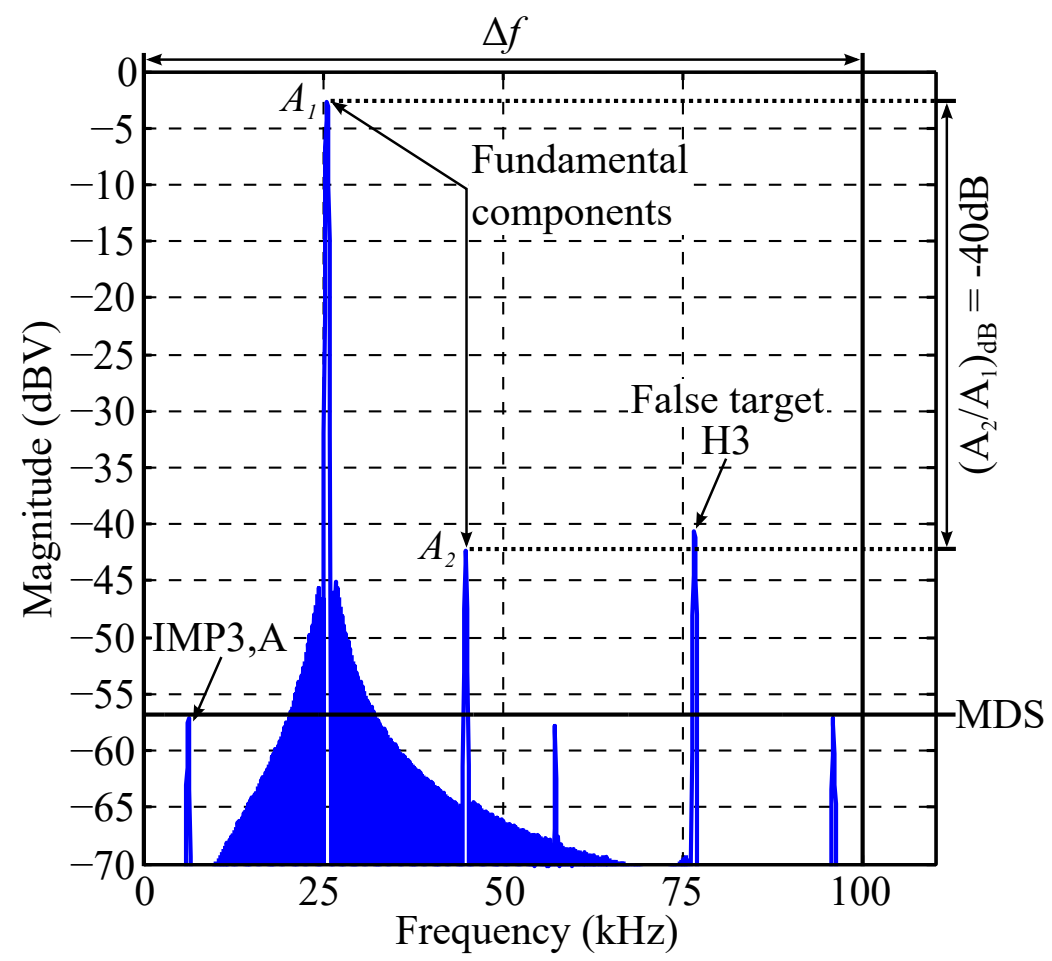

Figure 5.33: Baseband receiver spectrum, where the third order harmonic (H3) is detected as a false target.

\subsection{Conclusion}

It is shown that the RF front-end's dynamic range is truncated by the ADC's dynamic range. Therefore, the IF receiver's effective dynamic range is dictated by the ADC's 
performance.

Three components are investigated in the chapter: the LNA, IQ demodulator, and high-voltage gain op-amp. Due to the op-amp's high gain, it is first to saturate when a large reflection is received.

For multi-tone excitation a method is presented to predict the op-amp's non-linear behaviour. A model is used to investigate the effect multiple reflections and close range targets have on the FMCW radar receiver. It is concluded that the IF receiver is effective at removing harmonic distortions and that low order IMPs that are spectrally near to the fundamental components could be detected as false targets. The position of the IMPs are depend on the received signals causing the non-linearity. It is shown that the baseband receiver is susceptible to distortions the IF receiver can suppress. 


\section{Chapter 6}

\section{Receiver characteristics and dynamic range}

By comparing measured results to calculations from previous chapters the FMCW radar is characterised in terms of sensitivity, noise, dynamic range and selectivity.

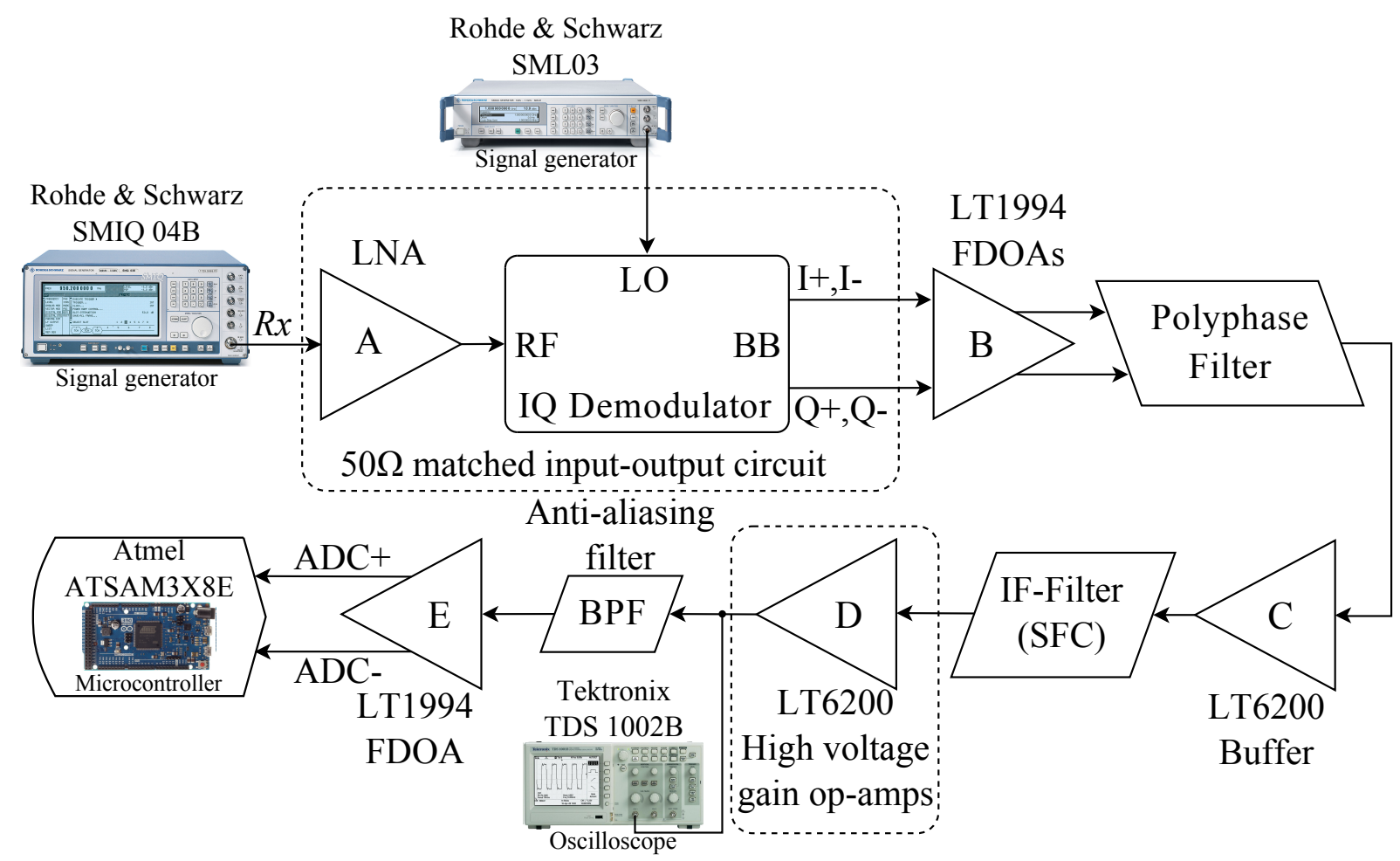

Figure 6.1: Receiver measurement set-up.

The measurement set-up used to characterise the receiver is shown in Fig. 6.1. A single tone is excited by the Rohde \& Schwarz (R\&S) SMIQ 04B signal generator at 601.045 MHz, while the R\&S SML03 generates a $600 \mathrm{MHz}$ LO. Therefore, the down converted I-and Q-channel signals, at an intermediate frequency (IF) of $1.045 \mathrm{MHz}$ are in the receiver's passband. 


\subsection{Windowing of discrete signals}

The spectrum of a quantised signal is generated using the FFT algorithm. For the algorithm it is assumed that the quantised signal repeats exactly throughout all time. Signals measured in the time domain are periodic at intervals that correspond to the length of the recorded time. If the sampled signal is not an integer number of cycles, the assumption is violated and spectral leakage occurs [61].

Spectral leakage distorts measurements because energy from a given frequency component is spread over adjacent frequency bins. Windowing is used to minimise the effect of spectral leakage [61].

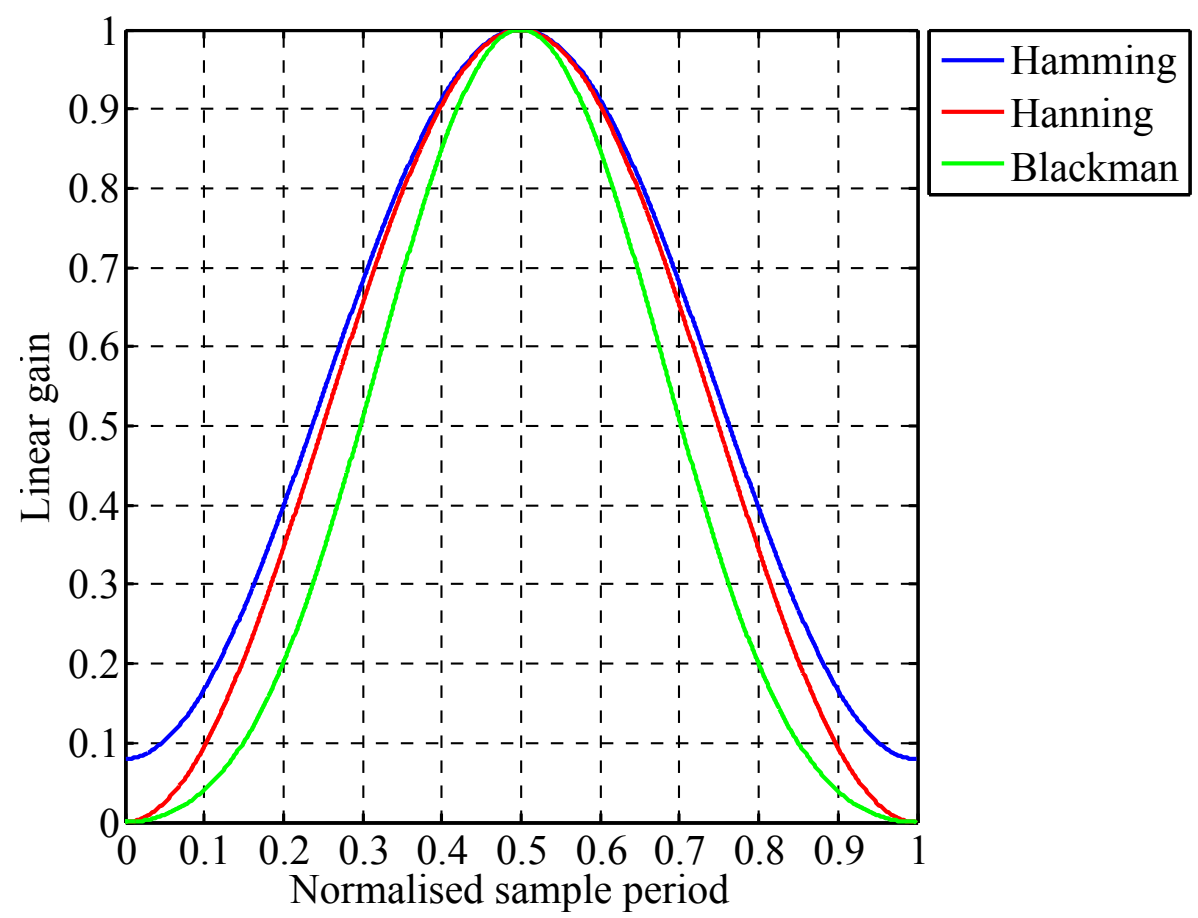

Figure 6.2: Three common windows to reduce spectral leakage.

The spectrum of a rectangular windowed cosine signal takes the from of a $\operatorname{sinc}(x)=$ $\frac{\sin (x)}{x}$ function according to Fourier theory. The largest side lobe of the uniform windowed signal is $13.2 \mathrm{~dB}$ below the main lobe. This causes problems when targets are close together because the side lobes of larger targets can conceal smaller targets. Figure 6.2 shows three commonly used windows, the Hamming, Hanning and Blackman. Their characteristics are presented in Table 6.1.

Table 6.1: Characteristics of common non-uniform windows

\begin{tabular}{c|c|c|c|c|c}
\hline \hline Window & Unit & Rectangular & Hamming & Hanning & Blackman \\
\hline Greatest side lobe & $\mathrm{dB}$ & -13.2 & -42.8 & -31.4 & -58 \\
3 dB lobe width & FFT-bins & 0.89 & 1.3 & 1.44 & 1.62 \\
SNR loss & $\mathrm{dB}$ & 0 & 1.34 & 1.76 & 2.37 \\
Main lobe width & FFT-bins & 2 & 4 & 4 & 6 \\
Side lobe roll-off rate & $\mathrm{dB} /$ decade & 20 & 20 & 60 & 60
\end{tabular}


Different windows are suited to different applications. For FMCW radar applications frequency resolution is important, so typically either the rectangular or Hamming windows are used. To reduce spectral leakage, the Hamming window is selected even though it degrades the FFT's resolution by a factor of 1.3 and the SNR by $1.34 \mathrm{~dB}$.

A windowing function is applied by multiplying its response with the acquired timedomain signal. This process introduces distortion because the window alters the overall amplitude of the signal. Windows are scaled by dividing the windowed array with the window's coherent gain [61].

Each window increases the effective bandwidth of an FFT bin by an amount known as the equivalent noise power bandwidth. The power of a given frequency peak is computed by adding the adjacent frequency bins around the peak. This inflates the result and is taken into account by dividing with the window's noise power bandwidth. The summation should span wider than the window's main lobe width [61]. The scaling factors and noise power bandwidths of commonly-used windowing functions are provided in Table 6.2.

Table 6.2: Window correction factors

\begin{tabular}{c|c|c|c|c}
\hline \hline Window & Rectangular & Hamming & Hanning & Blackman \\
\hline Scaling factor (coherent gain) & 1 & 0.54 & 0.5 & 0.42 \\
Noise power bandwidth & 1 & 1.36 & 1.5 & 1.71
\end{tabular}

\subsection{Receiver characterisation}

In this section measurements are presented to validate the receiver's characteristics as calculated in section 5.2 .

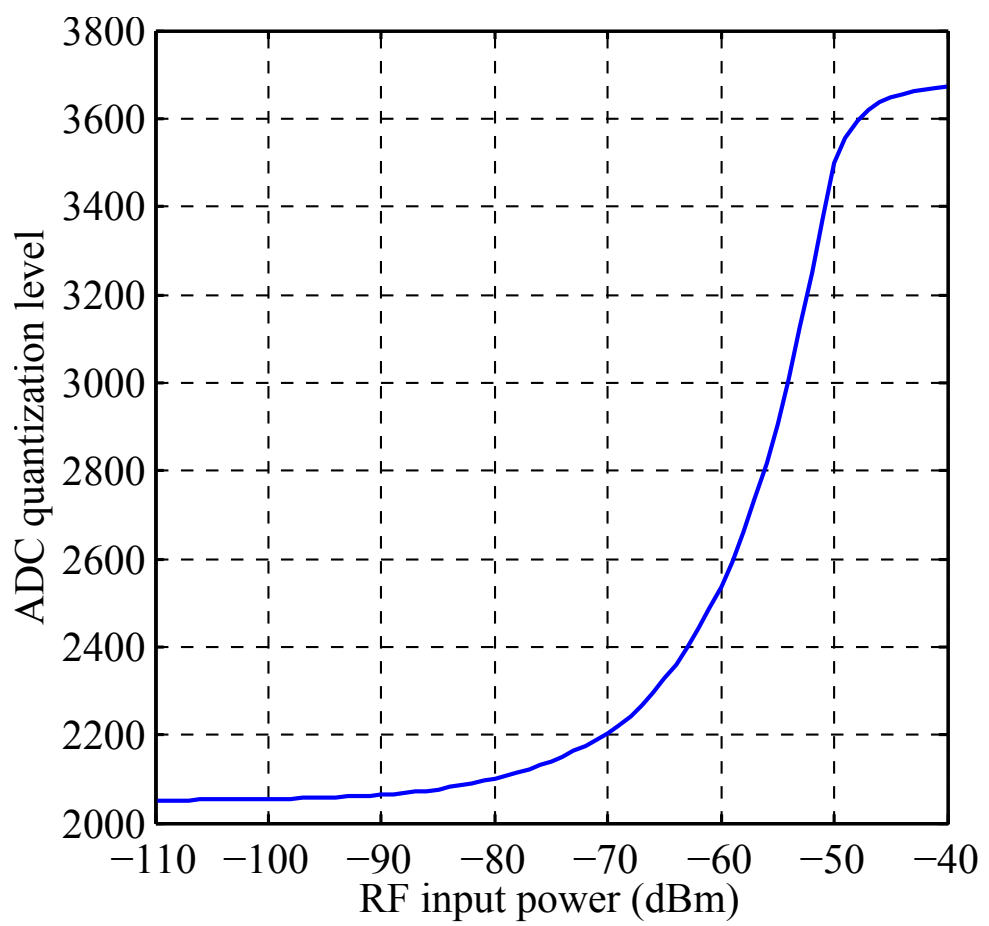

Figure 6.3: Signal amplitude in ADC quantisation levels versus RF input power in dBm. 


\subsubsection{Quantized signal linearity}

Figure 6.3 shows measured voltage amplitudes in terms of the ADC's quantisation levels for a single tone excitation at the receiver's RF front end. Due to receiver saturation, the quantised signal amplitude plateaus at RF input power levels above $-53 \mathrm{dBm}$. The signal compression point is clearly illustrated in Fig. 6.4. The 12-bit quantisation range is not entirely utilised. A safety margin exists to prevent the introduction of non-linearities when constructive interference is experienced under multi-tone excitation. Further optimisation and experimentation with regards to the optimal quantisation margin would yield better receiver performance and dynamic range.

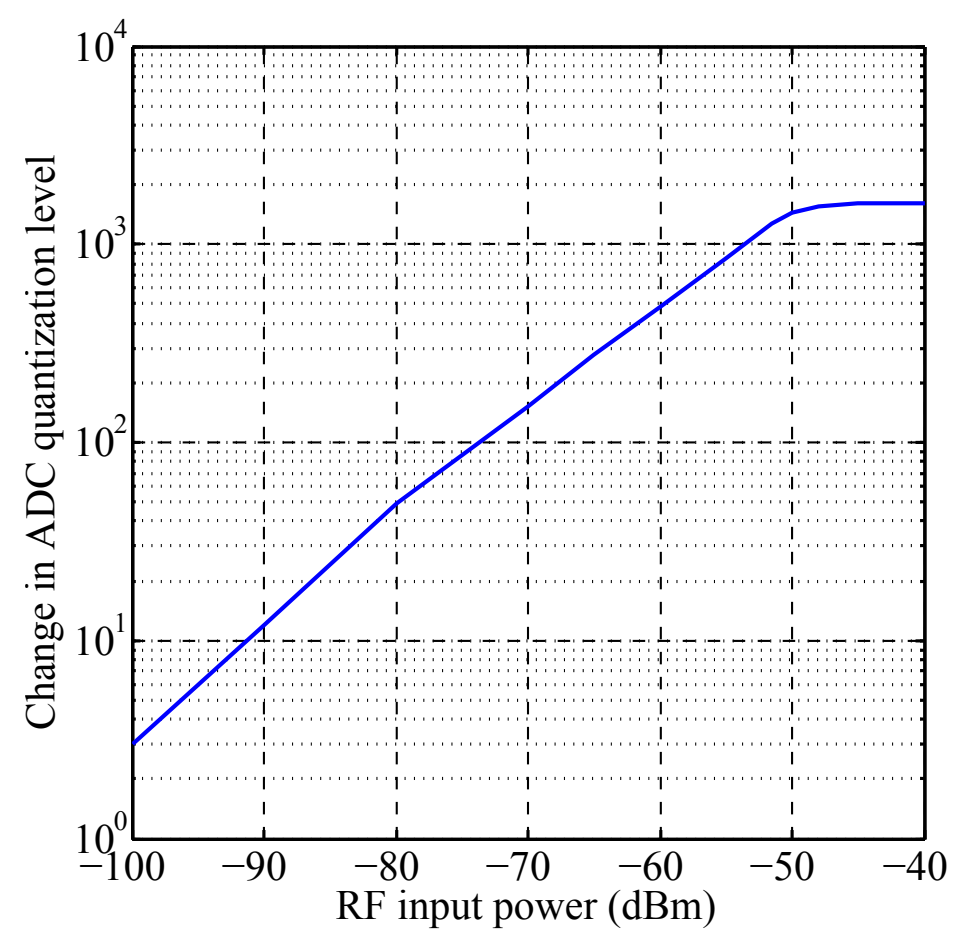

Figure 6.4: Change in the ADC's quantisation level on a logarithmic scale versus RF input power in $\mathrm{dBm}$.

\subsubsection{Receiver frequency response}

The receiver's amplitude response is measured by stepping the RF input signal's frequency from $0.8 \mathrm{MHz}$ to $1.4 \mathrm{MHz}$ while the R\&S FSEK30 spectrum analyser's maximum hold function is active, producing Fig. 6.5.

By knowing the receiver's passband gain and amplitude response, a quantised signal's RF input power level can be determined. Figure 6.5 illustrates the receiver's bandwidth, selectivity, and the total combined rejection by the IF and anti-aliasing filters. The effect the cascaded receiver network has on a signal's magnitude is described by the measurement. However, because of the spectrum analyser's finite dynamic range the normalised rejection is not measured below $-68 \mathrm{~dB}$. 


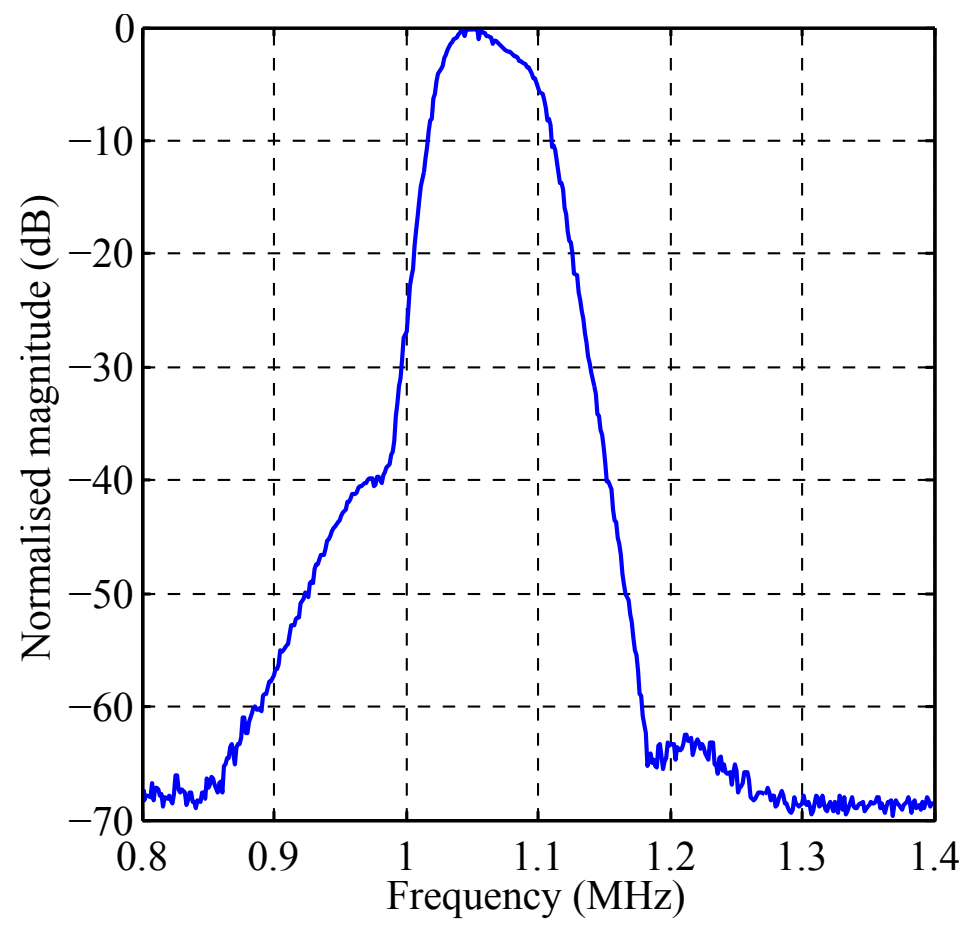

Figure 6.5: The receiver's normalised amplitude response.

\subsubsection{Measured receiver gain}

The receiver's voltage and power gain is calculated from the data depicted in Fig. 6.3. The MATLAB code used in this calculation is provided in Appendix B.7. Equation (6.2.2) describes the method used to translate the ADC's measured quantisation level to an equivalent input voltage amplitude,

$$
\begin{aligned}
L & =2^{12}=4096, \\
v_{A D C} & =\left(\frac{q}{L}-0.5\right) v_{r e f},
\end{aligned}
$$

where $q$ is the measured signal's amplitude in quantisation levels, $L$ is the full scale quantisation range, and $v_{r e f}$ is the ADC's reference voltage.

Each of the ADC's single ended channels quantise a voltage across a load impedance of $390 \Omega$. The equivalent power at the ADC's input is calculated from the discretised signals. The power gain is determined by calculating the ratio between the known RF input power and the input power measured by the ADC.

The voltage gain is determined by converting the applied RF power to an equivalent voltage across a $50 \Omega$ load. The gain plots seen in Fig. 6.6 are produced by taking the ratio between the measured input and output signals.

At RF input power levels above $-53 \mathrm{dBm}$, the measured gain decreases (see Fig. 6.6) because the receiver's high voltage gain amplifier ( $D$ in Fig. 6.1) begins to saturate. At RF excitations below $-90 \mathrm{dBm}$ the ADC's resolution is too low for accurate gain measurements. The average gain is calculated in the linear region, i.e. for measurements made at RF input power levels between $-90 \mathrm{dBm}$ and $-53 \mathrm{dBm}$. The measured and calculated gain (from section 5.2) are presented in Table 6.3. 


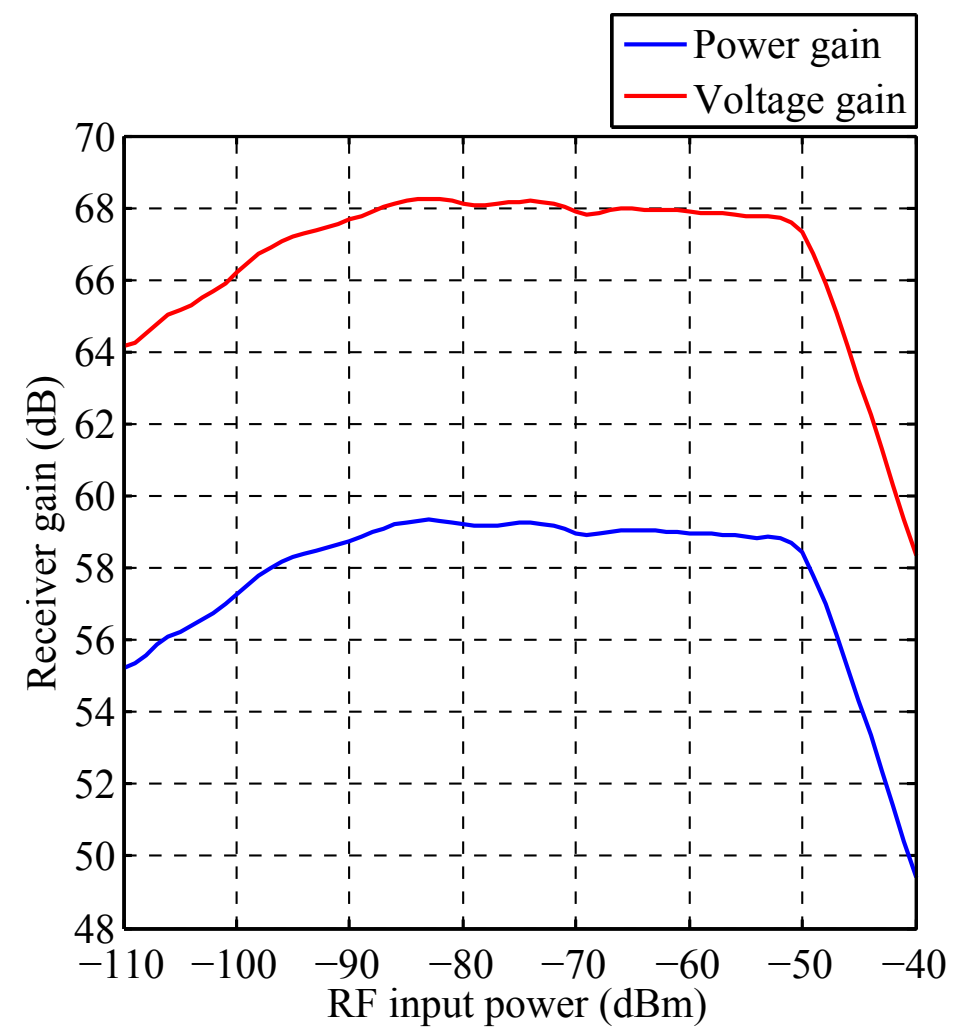

Figure 6.6: The measured voltage and power gain.

\subsubsection{Full scale input signal}

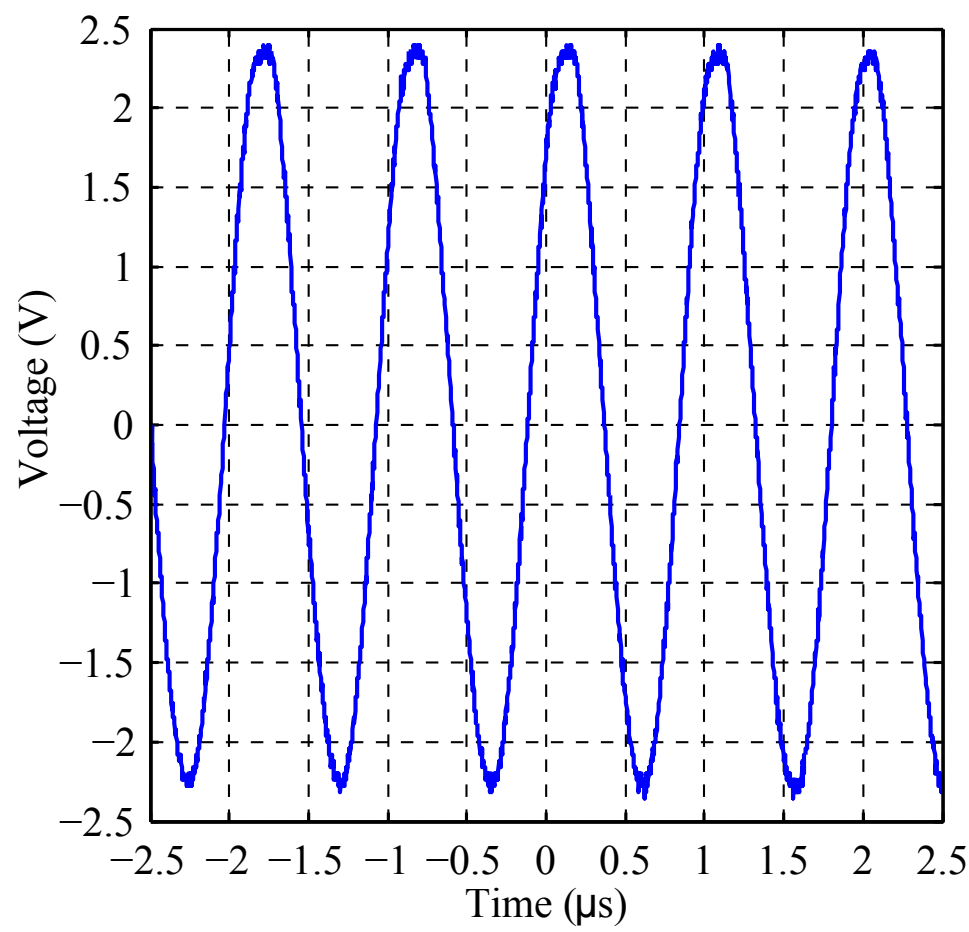

Figure 6.7: Time domain measurement of the LT6200 op-amp in saturation.

The full scale input power is measured at the receiver's $1 \mathrm{~dB}$ compression point. The first component to saturate is the high voltage gain op-amp, labelled D in Fig. 6.1. The 
Linear Technologies LT6200 op-amp limits the output signal's voltage swing at its positive and negative supply rails, generating undesired harmonics. The receiver's measured $1 \mathrm{~dB}$ compression point is at a single tone input power of $-53 \mathrm{dBm}$.

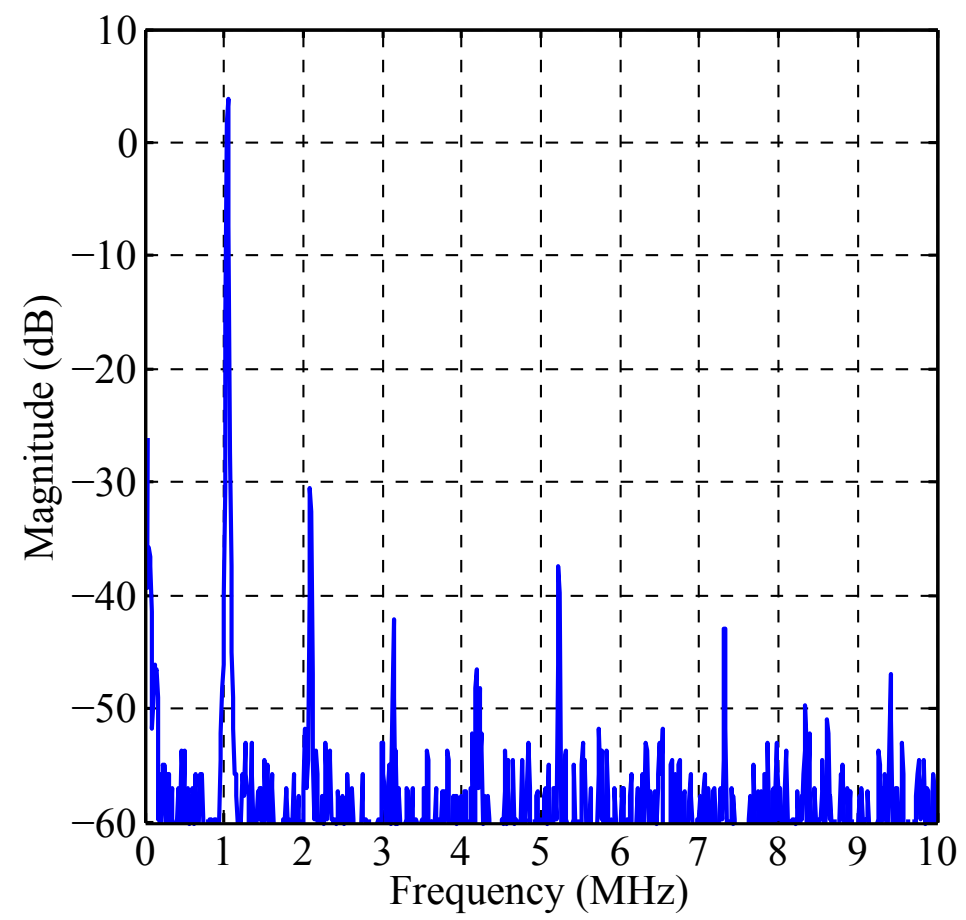

Figure 6.8: Frequency domain measurement that shows non-linearity at the LT6200 opamp's output.

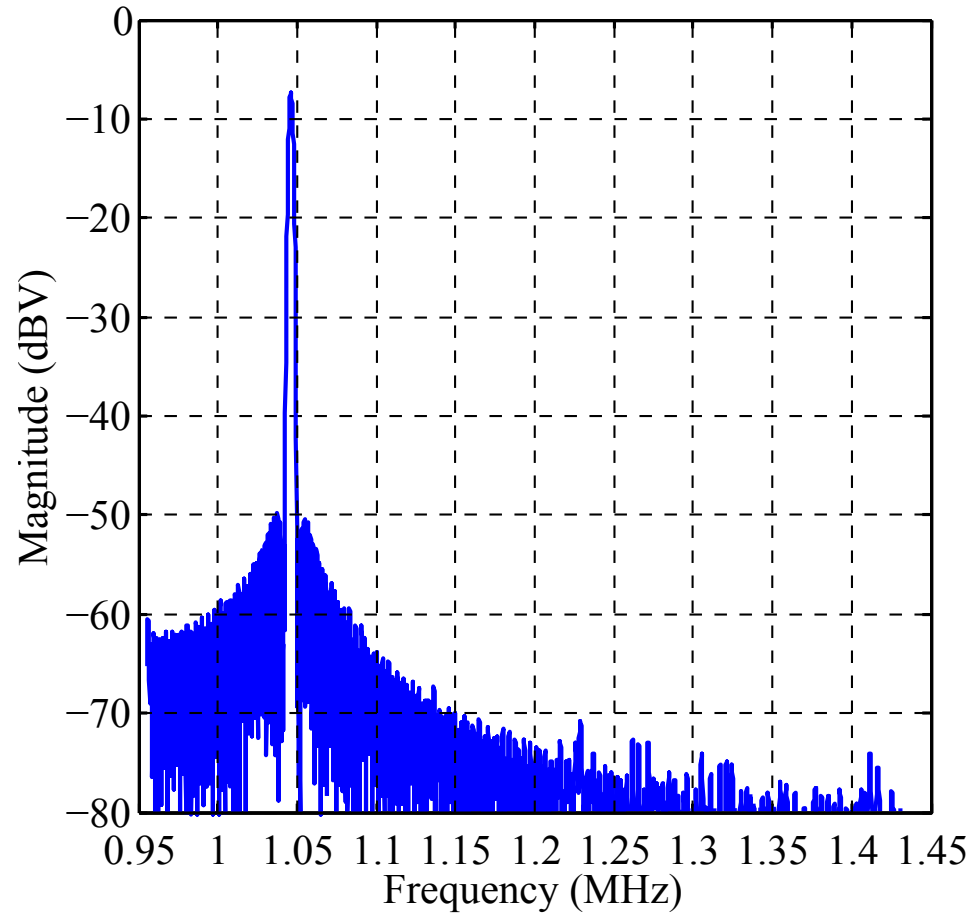

Figure 6.9: The undesired harmonics are physically removed by the anti-aliasing filter and a spurious free spectrum is digitised by the ADC. 
An RF signal of $-51.5 \mathrm{dBm}$ is applied to the radar front-end, causing the receiver to saturate. Time and frequency domain measurements are made using a Tektronix TDS1002B oscilloscope, the results are shown in Figs. 6.7 and 6.8 respectively. The harmonics are clearly visible in the frequency domain. The largest of the spurious signals is the second harmonic (at $2 f_{1}=2.09 \mathrm{MHz}$ ) that is $35 \mathrm{~dB}$ below the fundamental component (at $f_{1}=1.045 \mathrm{MHz}$ ).

For a zero-IF receiver the harmonics shown in Fig. 6.8 are detected as false targets. However, in the case of the IF receiver the harmonics are out-off band and thus suppressed by the anti-aliasing filter. The spectrum shown in Fig. 6.8 is filtered by the anti-aliasing filter and discretised by the ADC producing the spurious free spectrum in Fig. 6.9.

\subsubsection{Minimum detectable signal}

The MDS is defined as the smallest possible RF signal that results in an unambiguous detection by the ADC. The receiver's noise floor is measured by the MCU's ADC producing Fig. 6.10. The RF noise floor is calculated by subtracting the measured average power gain from the quantised spectrum.

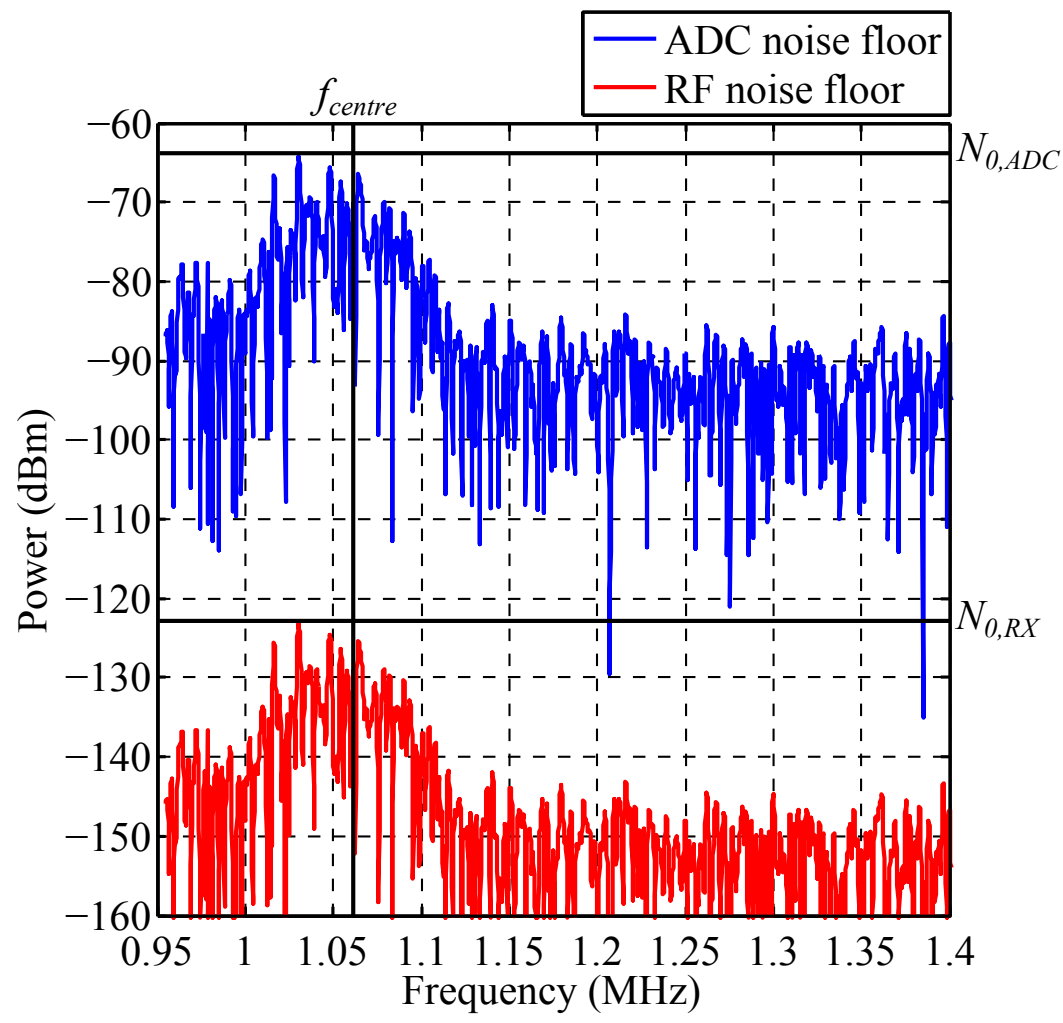

Figure 6.10: Measured noise floor.

The receiver's passband noise floor is

$$
\begin{aligned}
N_{0, A D C} & =-64.3 \mathrm{dBm}, \\
N_{0, R X} & =-123.3 \mathrm{dBm} .
\end{aligned}
$$

The IF filter's centre frequency is indicated in Fig. 6.10. The MDS is determined by adding the measured passband noise figure of $14.5 \mathrm{~dB}$ to the RF noise floor

$$
M D S_{R X}=N_{0, R X}+N F=-108.8 \mathrm{dBm} .
$$




\subsubsection{Receiver dynamic range}

The receiver's dynamic range is calculated as described in section 5.2 by using Eq. (6.2.5) and the measurement results from sections 6.2.4 and 6.2.5.

$$
D R=F S_{R X}-M D S_{R X}
$$

Table 6.3: Receiver characteristics - measured and calculated results

\begin{tabular}{c|c|c|c|c}
\hline \hline Description & Symbol & $\begin{array}{c}\text { Measured } \\
\text { value }\end{array}$ & $\begin{array}{c}\text { Calculated } \\
\text { value }\end{array}$ & Unit \\
\hline Power gain & $G_{c a s}$ & 59 & 57.3 & $\mathrm{~dB}$ \\
Voltage gain & $G_{v, c a s}$ & 68 & 66.3 & $\mathrm{~dB}$ \\
Full scale RF input & $F S_{R X}$ & -53 & -51.9 & $\mathrm{dBm}$ \\
ADC full scale input & $F S_{A D C}$ & 0.4 & 1.3 & $\mathrm{dBV}$ \\
RF MDS & $M D S_{R X}$ & -108.8 & -110.1 & $\mathrm{dBm}$ \\
ADC MDS & $M D S_{A D C}$ & -55.4 & -56.8 & $\mathrm{dBV}$ \\
\hline Dynamic range + window SNR loss & $D R_{w i n}$ & 55.8 & 58.2 & $\mathrm{~dB}$ \\
Dynamic range & $D R$ & 57.1 & 58.2 & $\mathrm{~dB}$ \\
\hline
\end{tabular}

The variables that characterise the receiver are shown in Table 6.3 and both the measured and calculated values are presented. To compensate for the loss in signal-tonoise ratio caused by the Hamming window, $1.34 \mathrm{~dB}$ is added to the measured dynamic range.

\subsubsection{Averaging over multiple modulation periods}

By averaging the received signals over multiple modulation periods the signal-to-noise ratio is improved. Averaging lowers the signal strength of uncorrelated signals from one period to the next. Therefore, noise is reduced because the noise floor and quantisation noise is mostly the product of uncorrelated noise sources [62].

With regards to averaging, the modular design presented in this study is not ideally suited to the undersampling application used by the receiver. Synchronization issues are experienced due to the MCU and DDS running from separate clocks. The MCU triggers the DDS to generate a chirp, but the point at which the MCU's ADC starts to sample and the DDS chirp signal begins is different from one modulation cycle to the next. SuperNyquist sampling exacerbates the synchronisation problem, making it virtually impossible to average over multiple modulation periods.

The solution is further system integration by running the ADC and DDS from a single clock source.

\subsection{Demodulator LO to RF leakage}

A demodulator has limited isolation between its RF and LO ports. Consequently, some of the LO signal leaks to the RF port and visa versa. The LO to RF leakage of the Analog Devices ADL5380 is measured and the results shown in Table 6.4. For the measurement the RF balun (Mini-circuits TC1-1-13M+) and cable losses are de-embedded. 
Table 6.4: LO leakage to RF port of the ADL5380 demodulator

\begin{tabular}{c|c}
\hline \hline LO to RF leakage $(\mathrm{dBm})$ & LO frequency $(\mathrm{MHz})$ \\
\hline-53.6 & 400 \\
-52.9 & 500 \\
-52.0 & 600 \\
-51.5 & 700 \\
-50.8 & 800 \\
\hline
\end{tabular}

The measured LO to RF leakage has a flat response within the bandwidth of interest (400 MHz to $800 \mathrm{MHz}$ ). The demodulator has internal amplifiers that buffer the LO signal before it is applied to the device's internal fully-balanced mixers. The leakage level does not notability vary with a change in LO power level because the LO signal amplitude is limited by the internal $\mathrm{LO}$ amplifiers.

If the RF port is connected to a poorly matched source (e.g. antenna, LNA) some of the leaked signal energy is reflected back to the demodulator's RF port and mixed down to baseband as illustrated in Fig. 6.11 . The baseband beat frequency $\left(f_{L O, 1}\right)$ of the resultant spurious signal depends on the length of cable between the mismatched ports.

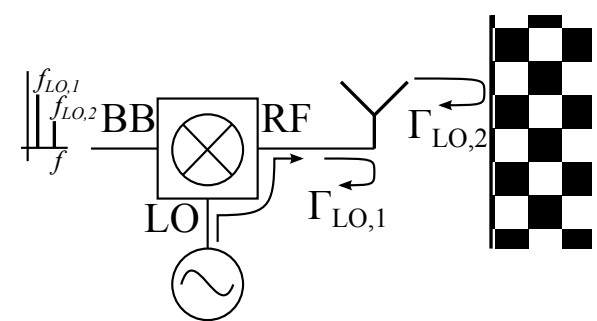

Figure 6.11: False targets caused by LO to RF leakage.

Assume that a matched antenna is connected to the demodulator's RF port (i.e. no LNA to increase the isolation between the antenna and demodulator) and the antenna is held close to a target surface. The signal leakage from the LO to RF port is transmitted and then received by the same antenna at the demodulator's RF port. Due to the time delay caused by signal propagation, the reflection is mixed down to baseband at a beat frequency related to the delay $\left(f_{L O, 2}\right)$.

This scenario is problematic for receivers mixing down to baseband because these spurious signals are not mixed to the IF, therefore, an IF receiver is immune to this issue.

\subsection{Conclusion}

Good agreement is obtained between the calculated and measured results presented in Table 6.3. It is found that the receiver has the potential for greater dynamic range with further optimisation and experimentation. Section 6.2.4 shows that it is possible to remove unwanted harmonics when a single tone RF input causes the receiver's high gain op-amp to saturate.

The effect of the demodulator's LO to RF leakage on the FMCW radar's performance is discussed in section 6.3 and it is argued that using an IF receiver negates these effects. 


\section{Chapter 7}

\section{Measurements and results}

The procedure followed to measure the receiver's noise figure is discussed in this chapter. Measurement results are presented to illustrate the IF receiver's ability to reject harmonics and IMPs.

\subsection{Receiver noise figure measurement}

Noise is usually measured at such low power levels that it is assumed that the components in the receiver chain behave in an entirely linear manner. Consider the case where the applied input signal is zero but the device under test (DUT) is loaded by a source impedance. The power presented to the DUT is the thermal noise generated by the source impedance. If the DUT is linear, the output power characteristic versus source temperature has a linear relationship, as illustrated by Fig. 7.1.

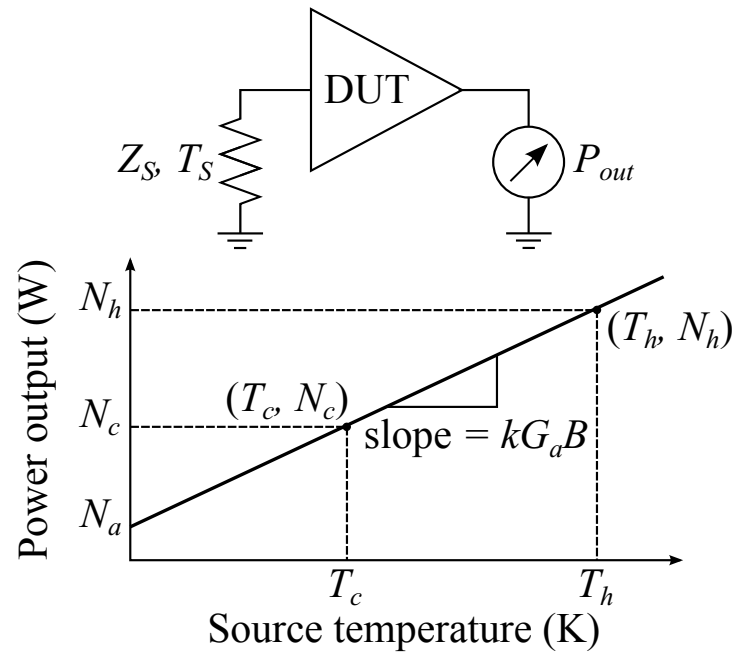

Figure 7.1: Output noise power versus noise source temperature.

For a source temperature of zero Kelvin (i.e. at the origin of the x-axis in Fig. 7.1), the only noise measured at the device output is the noise generated by the DUT and is represented by the symbol $N_{a}$. At any other source temperature $T_{s}$, an input noise power density $k T_{s}$ (in $\mathrm{W} / \mathrm{Hz}$ ) is generated by electron vibration in the source. The input noise power is amplified by the DUT's gain $\left(G_{a}\right)$ producing additional output noise, resulting 
in a total output noise power of

$$
N_{t}=N_{a}+k T_{s} G_{a} \Delta f
$$

where $\Delta f$ is the DUT's bandwidth.

By knowing two points along the noise characteristic curve the noise figure and effective input noise temperature are determined. In Fig. 7.1 two temperatures are indicated, the cold measurement $T_{c}$ with a corresponding noise power $N_{c}$ and the hot measurement $T_{h}$ producing an output noise power of $N_{h}$. The ratio $N_{h} / N_{c}$ is called the Y-factor [8].

\subsubsection{Y Factor method}

The IF receiver's noise figure is measured using the $\mathrm{Y}$ factor method. For this method, a noise source with a hot and cold temperature $\left(T_{h}\right.$ and $T_{c}$ respectively) are required. Noise source manufacturers usually specify the noise temperature in terms of the excess noise ratio (ENR),

$$
\mathrm{ENR}_{d B}=10 \log \left(\frac{T_{h}-T_{c}}{T_{0}}\right)
$$

where $T_{0}$ and $T_{c}$ are assumed to be $290 \mathrm{~K}$ for a calibrated noise source. ENR is the ratio expressed in $\mathrm{dB}$ of the temperature change between the noise source's on $\left(T_{h}\right)$ and off $\left(T_{c}\right)$ states divided by $T_{0}$ [63].

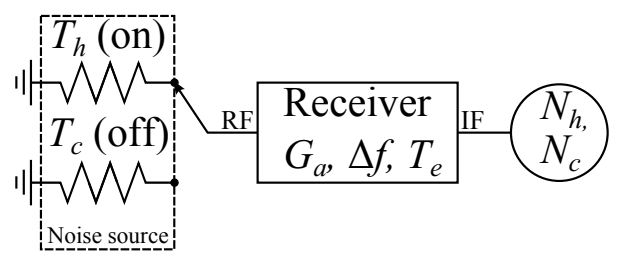

Figure 7.2: Test set-up for the $\mathrm{Y}$ factor noise figure measurement method.

Figure 7.2 illustrates the set-up for the $\mathrm{Y}$ factor noise figure (NF) measurement [8]. The noise figure is determined as follows

$$
N F=10 \log \left[\frac{\operatorname{ENR}-Y\left(\frac{T_{c}}{T_{0}}-1\right)}{Y-1}\right],
$$

and $Y=\frac{N_{h}}{N_{c}}$ [63]. An Agilent Technologies 346B noise source is used during the measurement. The ENR values supplied by the manufacturer is provided in Table 7.1. These values are used to determine $T_{h}$ at the receiver's IF and $T_{c}$ is taken at room temperature $\left(T_{0}\right)$, simplifying Eq. (7.1.3) to

$$
N F=10 \log \left(\frac{\mathrm{ENR}}{Y-1}\right) .
$$

In most cases the noise source is used with an accompanying noise figure meter. However, the noise figure meter is not capable of measurements below $10 \mathrm{MHz}$ and the IF receiver operates around $1 \mathrm{MHz}$ after down converting. Therefore a different approach is followed to determine the receiver's noise figure. 
Table 7.1: ENR values for the Agilent Technologies 346B noise source.

\begin{tabular}{c|c}
\hline \hline Frequency $(\mathrm{MHz})$ & ENR $(\mathrm{dB})$ \\
\hline 10 & 14.94 \\
1000 & 14.95 \\
2000 & 14.85 \\
3000 & 14.75 \\
4000 & 14.69 \\
5000 & 14.68 \\
\hline
\end{tabular}

An alternative to using the noise figure meter is measuring the noise power at the DUT's output using an ADC as illustrated by Fig. 7.3. The Tektronix TDS 1002B oscilloscope is an 8-bit ADC with a 2500 word buffer. The oscilloscope's data output is read into MATLAB where the sampled noise power is averaged at each frequency point. The receiver output is applied to an amplifier with $50 \mathrm{~dB}$ voltage gain. It ensures that the measured noise power is higher than the noise floor of the oscilloscope. The $50 \mathrm{~dB}$ amplifier is implemented by connecting two $25 \mathrm{~dB}$ decoupled op-amp stages in cascade. It is assumed that the receiver gain $\left(G_{a}\right)$ is high enough that the $50 \mathrm{~dB}$ amplifier's noise figure contribution is negligible (see section 4.3.1).

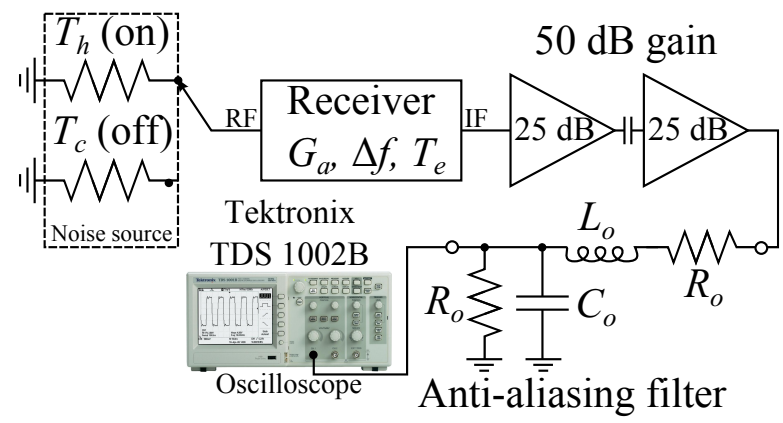

Figure 7.3: Test set-up for the Y factor noise figure measurement using an oscilloscope.

The frequency resolution of the FFT calculated from the averaged oscilloscope measurements is determined by dividing the sampling frequency by the oscilloscope's buffer size $\left(\frac{f_{s}}{2500}\right)$. The oscilloscope is set-up to sample at $5 \mathrm{MHz}$, thus the spectral resolution is $2 \mathrm{kHz}$ and the Nyquist frequency is $2.5 \mathrm{MHz}\left(\frac{f_{s}}{2}\right)$. Signal aliasing is prevented by a first order low pass filter designed with a $1.2 \mathrm{MHz}$ cut-off frequency.

The measured noise figure is shown in Fig. 7.4 alongside the noise figure calculated in Chapter 4 . The measured and calculated results show good agreement for noise figures below $20 \mathrm{~dB}$. During the calculation the IF filter's frequency response is taken into account, but the bandwidth limitations of the op-amps, peripheral RC filters, and the anti-aliasing filter are not included in the calculation. The measurement's resolution is limited by the oscilloscope's ADC. The measurement becomes less reliable at higher noise figure values because the receiver's attenuation is high and the signals are below the ADC's LSB. Using the noise figure measurement set-up discussed in this section it is possible to distinguish noise figure values up to $20 \mathrm{~dB}$. 


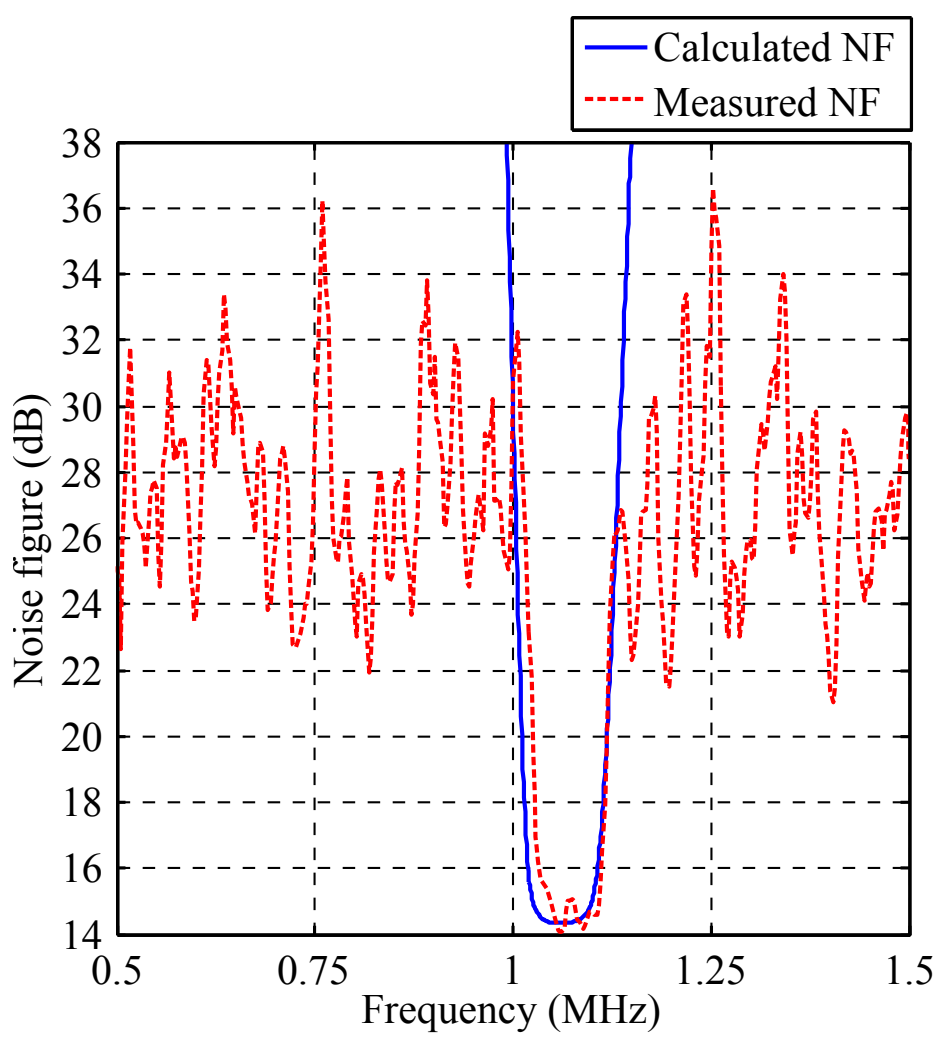

Figure 7.4: Calculated and measured cascaded noise figure versus frequency.

\subsection{Time domain interferometry measurements}

To assess the FMCW radar's non-linear behaviour and range finding capability a $50 \Omega$ coaxial transmission line is connected between the transmitter $(\mathrm{Tx})$ and the receiver $(\mathrm{Rx})$. The transmission power, modulation scheme and cable characteristics are known, consequently, the received signals are well defined. The interpretation of results are simplified by this controlled set-up.

The transmission line characteristics are: insertion loss $(\alpha)$, relative dielectric constant $\left(\epsilon_{r}\right)$ and length $(R)$. For the measurements a sawtooth modulation scheme with a modulation period $\left(T_{m}\right)$ of $1.1 \mathrm{~ms}$ and $400 \mathrm{MHz}$ bandwidth $(\Delta f)$ is generated by the DDS. Equation (1.3.5) from Chapter 1 is adjusted to calculate the beat frequency for the propagation delay in a coaxial transmission line in one direction from the transmitter to receiver,

$$
f_{b}=\frac{R \Delta f}{v_{p} T_{m}} .
$$

The propagation velocity $\left(v_{p}\right)$ in a coaxial line with an inner dielectric insulator made of Teflon $\left(\epsilon_{r}=2.1\right)$ is

$$
v_{p}=\frac{c_{0}}{\sqrt{\epsilon_{r}}}
$$

where $c_{0}$ is the speed of light in a vacuum.

For a chirp duration of $T_{m}$, the spectrum of the beat signal is resolved to an accuracy of

$$
\begin{aligned}
\delta f_{b} & =\frac{1}{T_{m}}, \\
& =909 \mathrm{~Hz},
\end{aligned}
$$


where $\delta f_{b}$ is the resolution bandwidth [5]. Substituting $\delta f_{b}$ into Eq. (7.2.1) produces the range resolution for the measurements

$$
\begin{aligned}
\delta R & =\frac{v_{p}}{\Delta f}, \\
& =0.52 \mathrm{~m} .
\end{aligned}
$$

The FFT's bin resolution is related to the ADC's sample frequency and the number of samples collected during the modulation period by

$$
\begin{aligned}
\delta f_{\text {bin }} & =\frac{f_{s}}{2} \frac{T_{s}}{T_{m}}, \\
& =455 \mathrm{~Hz} / \text { bin. }
\end{aligned}
$$

As discussed in section 6.1 a Hamming window is used to limit spectral leakage. This windowing function is applied to the measurements presented in this section.

\subsubsection{Single target}

A single target is emulated by connecting a 14 meter length of coaxial transmission line between the IF FMCW radar's transmitter and receiver as illustrated in Fig. 7.5. The DDS produces $0 \mathrm{dBm}$ at the transmitters output and by including the losses due to the cable and tunable attenuator the received signal power is determined. The tunable attenuator is used to set-up different test conditions.

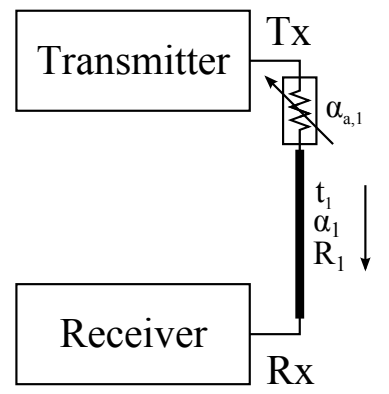

Figure 7.5: Single coaxial transmission line connected between the transmitter and receiver.

The received signal power is given by

$$
P_{R x}=P_{T x}+\alpha_{t}
$$

where $P_{T x}$ is the transmission power of $0 \mathrm{dBm}$ and the total insertion loss is

$$
\alpha_{t}=\alpha_{1}+\alpha_{a, 1}=-23 \mathrm{dBm},
$$

thus $P_{R x}=-23 \mathrm{dBm}$. The received power is below the RF front-end's $1 \mathrm{~dB}$ compression point (of $-3.9 \mathrm{dBm}$ calculated in section 2.1.3). Figure 7.6 (b) illustrates where the received signal is located on the IF filter's SFC. Due to SFC the down converted signal is attenuated by $25 \mathrm{~dB}$ relative to the IF filter's passband. By including the effect of SFC the received signal magnitude relates to $-48 \mathrm{dBm}$ in the IF filter's passband. 


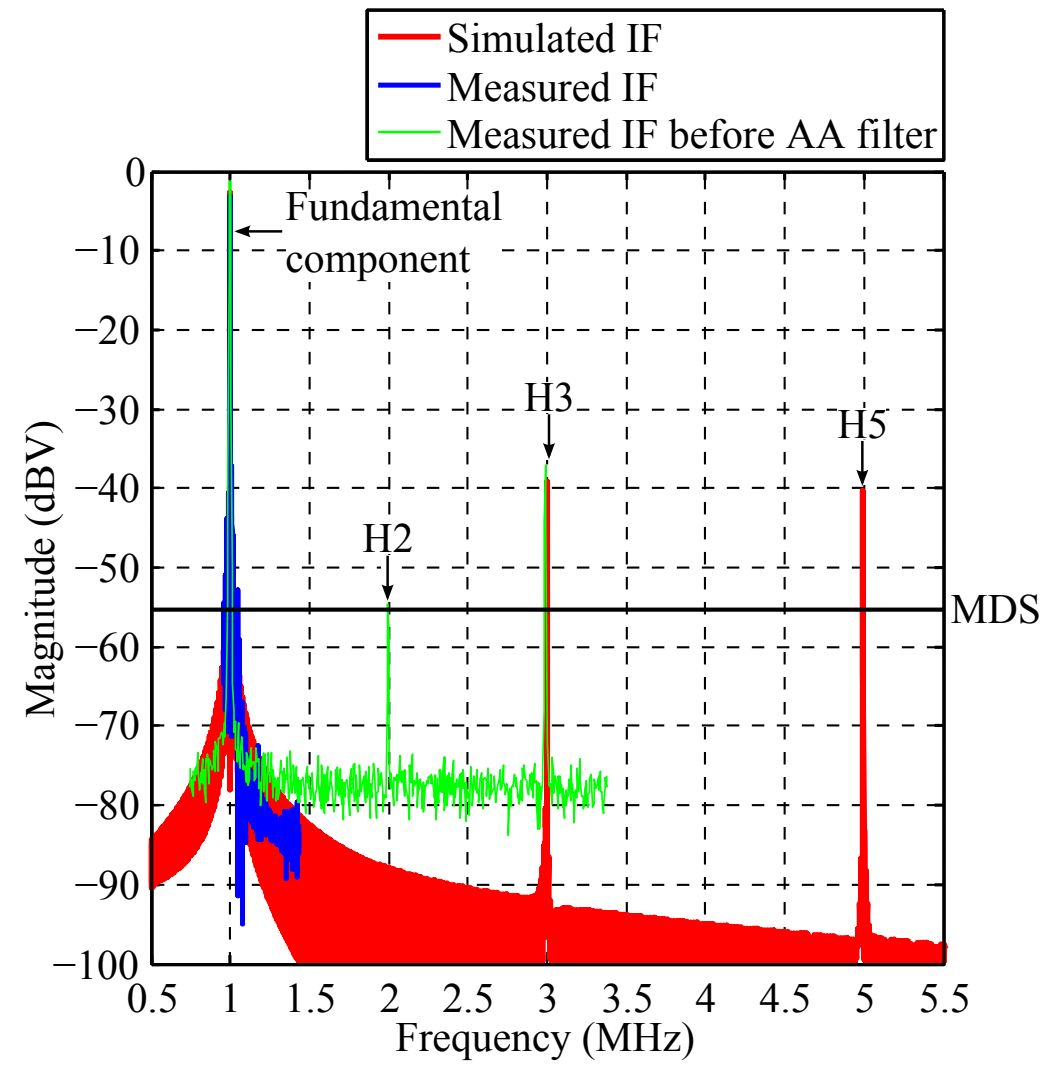

(a)

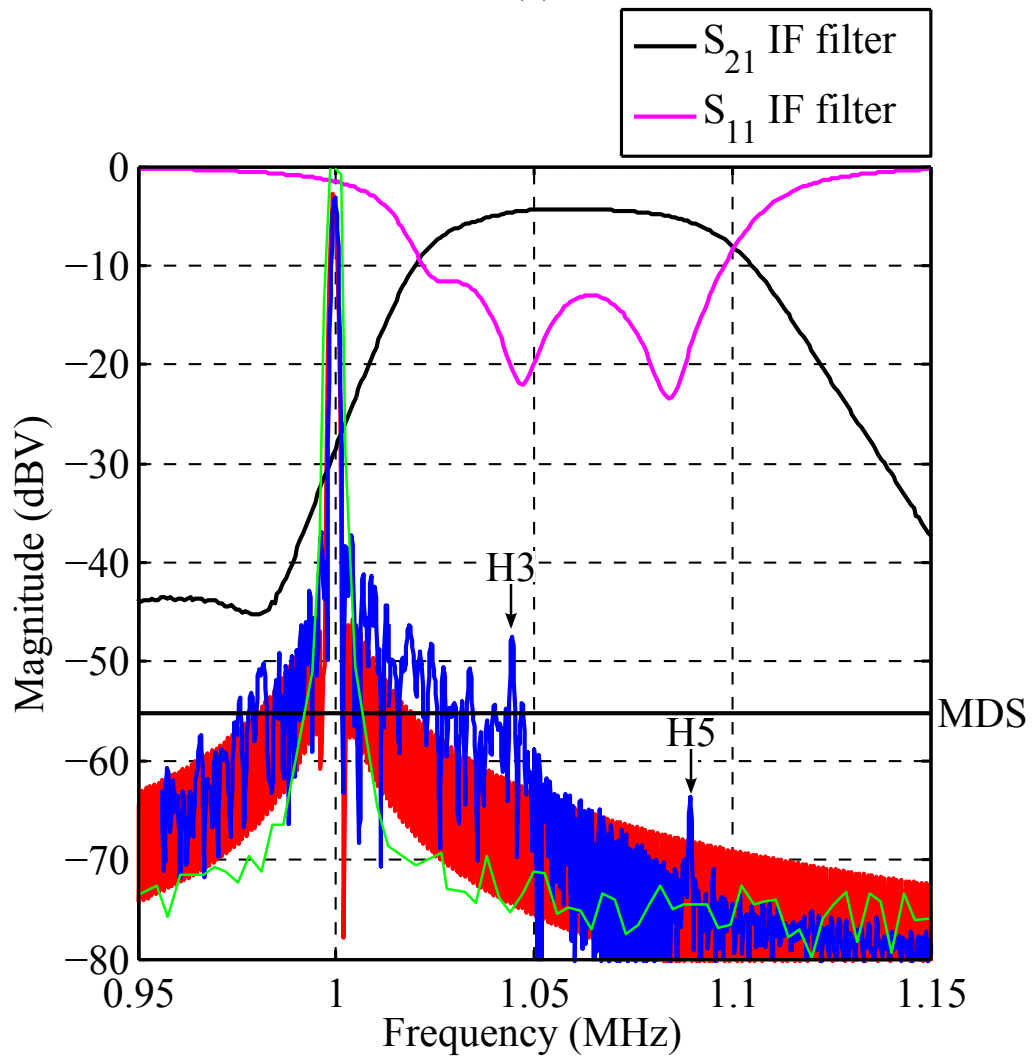

(b)

Figure 7.6: (a) Measured and simulated spectrum due to a single tone excitation $\left(P_{R x}=-23 \mathrm{~dB}\right)$. (b) Close-up spectral view of the measurements, simulation and IF filter response. 


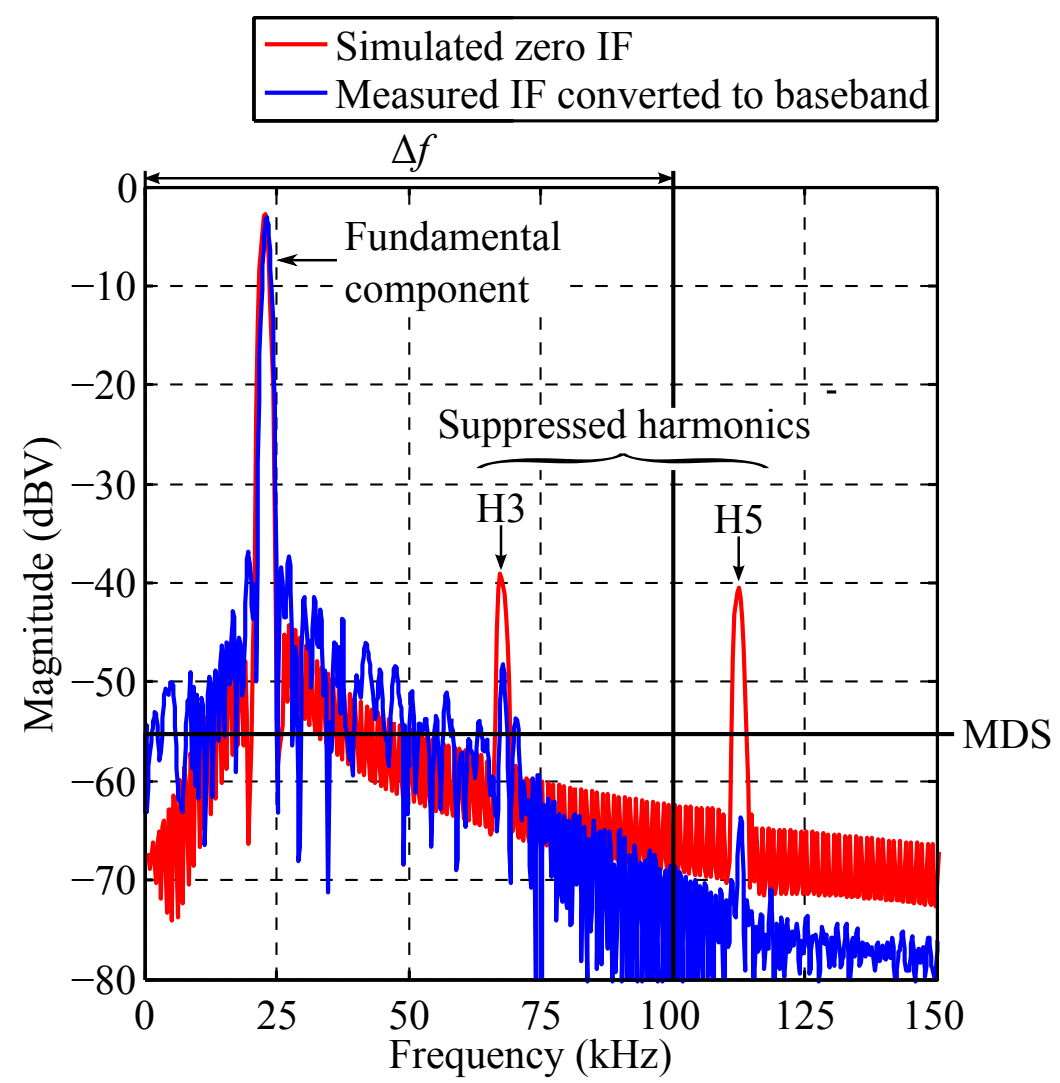

Figure 7.7: A single tone $\left(P_{R x}=-23 \mathrm{~dB}\right)$ zero-IF receiver simulation is shown along side a measurement made using the IF FMCW radar. The measured spectrum is transposed to baseband by subtracting the IF.

The dynamic range calculations in Chapter 5 show that a signal of $-48 \mathrm{dBm}$ in the receiver's passband will cause the receiver to saturate. The measured and simulated results for the IF receiver exposed to this single tone excitation is presented in Fig. 7.6. The figure shows the IF receiver saturating causing the generation of harmonics, where the $2 \mathrm{nd}$, 3rd and 5th harmonics exist around $2 \mathrm{MHz}, 3 \mathrm{MHz}$ and $5 \mathrm{MHz}$ respectively. The receiver's saturation is measured before and after the anti-aliasing (AA) filter respectively. The simulated IF receiver only produces uneven harmonics because it simulates symmetrical saturation. (The simulation and non-linear model used in this section is described in Chapter 5).

The IF receiver's ability to suppress harmonics is best illustrated at baseband to compare its performance to the zero IF receiver. The measured spectrum is transposed to baseband by subtracting the IF. This is compared to the zero-IF receiver simulation, as shown in Fig. 7.7, where both receivers are excited by the same input signal. From Fig. 7.7 it is clear that the harmonics are suppressed by the IF receiver, where the zero-IF receiver detects the third order harmonic (H3) as a false target. For the zero-IF receiver the third order harmonic's magnitude is above the MDS level while the distortion is in the receiver's passband.

In Figs. 7.6 (b) and 7.7 a 3rd and 5th harmonic is seen in the measurement made after the anti-aliasing filter. These harmonics are due to the ADC saturating and can be prevented by increasing the ADC's quantisation margin.

By applying Eq. (7.2.1) the beat frequency $\left(f_{b, 1}\right)$ due to the propagation delay in the 14 meter coaxial line is calculated, producing $f_{b, 1}=24.6 \mathrm{kHz}$. The baseband spectrum 
shown in Fig. 7.7 is equal to the beat frequency, i.e. the fundamental signal at $24 \mathrm{kHz}$ is also a result of the propagation delay in the transmission line. Given that the resolution bandwidth is almost $1 \mathrm{kHz}$ the difference in the calculated and measured beat frequency is acceptable.

\subsubsection{Two targets}

Two coaxial transmission lines are connected between the transmitter and receiver, as illustrated in Fig. 7.8, where the shorter coaxial line is 14 meters $\left(R_{1}\right)$ and the longer line 25 meters $\left(R_{2}\right)$. Due to the difference in the cable lengths each signal path has an insertion loss $\left(\alpha_{1}, \alpha_{2}\right)$ and propagation delay $\left(t_{1}, t_{2}\right)$ that results in two beat frequencies $\left(f_{b, 1}, f_{b, 2}\right)$. A power splitter and combiner are used to connect the coaxial cables in parallel, adding an additional 0.5 meters of cabling to each signal path.

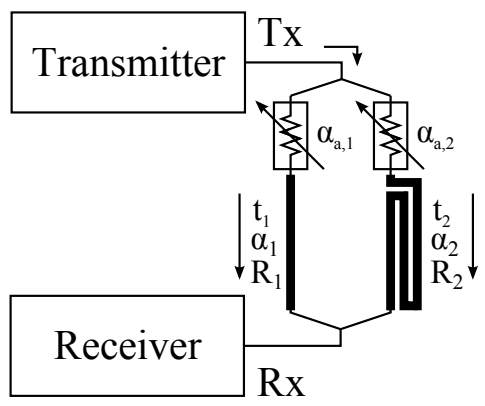

Figure 7.8: Parallel transmission lines connected between the transmitter and receiver.

Equation (7.2.1) is used to predict the beat frequency of each signal path. Resulting in $f_{b, 1}=25.5 \mathrm{kHz}$ and $f_{b, 2}=44.8 \mathrm{kHz}$ for the short and long transmission lines respectively. The two dominant frequency components at $25 \mathrm{kHz}$ and $43 \mathrm{kHz}$, seen in Figs. 7.10 (a) and 7.10 (b), are the measured beat frequencies. Acceptable agreement is held between the measured and calculated results when accounting for the radar's resolution bandwidth.

A two tone measurement is set-up that presents two target signals to the receiver, with power levels of $P_{R x, 1}=-23 \mathrm{dBm}$ and $P_{R x, 2}=-48 \mathrm{dBm}$ respectively $\left(\alpha_{t, 1}=-23 \mathrm{~dB}\right.$ and $\alpha_{t, 2}=-48 \mathrm{~dB}$ ). Taking SFC into account, the received signal strength is $A_{1}=-45$ $\mathrm{dBm}$ and $A_{2}=-55 \mathrm{dBm}$ when related to the IF filter's passband.

Figure 7.9 (a) illustrates the position of harmonics and IMPs for the saturated IF receiver. The filtered and quantised signals are shown in Fig. 7.9 (b). Two prominent 3rd order IMPs are located at $f_{I M P 3, A}=984 \mathrm{kHz}$ and $f_{I M P 3, B}=1.038 \mathrm{MHz}$ respectively.

As discussed in Chapter 5, the IMPs are a product of the mixing action between the two fundamental signals caused by the receiver's non-linearity during saturation. The 3rd order IMPs are spectrally near to the fundamental signals and would be detected as false targets by both the IF and zero-IF receivers. These components are located at

$$
\begin{aligned}
& f_{I M P 3, A}=2 f_{1}-f_{2}, \\
& f_{I M P 3, B}=2 f_{2}-f_{1},
\end{aligned}
$$

where $f_{1}$ and $f_{2}$ are the frequencies of the received echoes. The 5 th order IMP indicated at $f_{I M P 5, A}=3 f_{1}-2 f_{2}$ in Fig. 7.9 (b) is below the IF and outside the receiver's passband. 


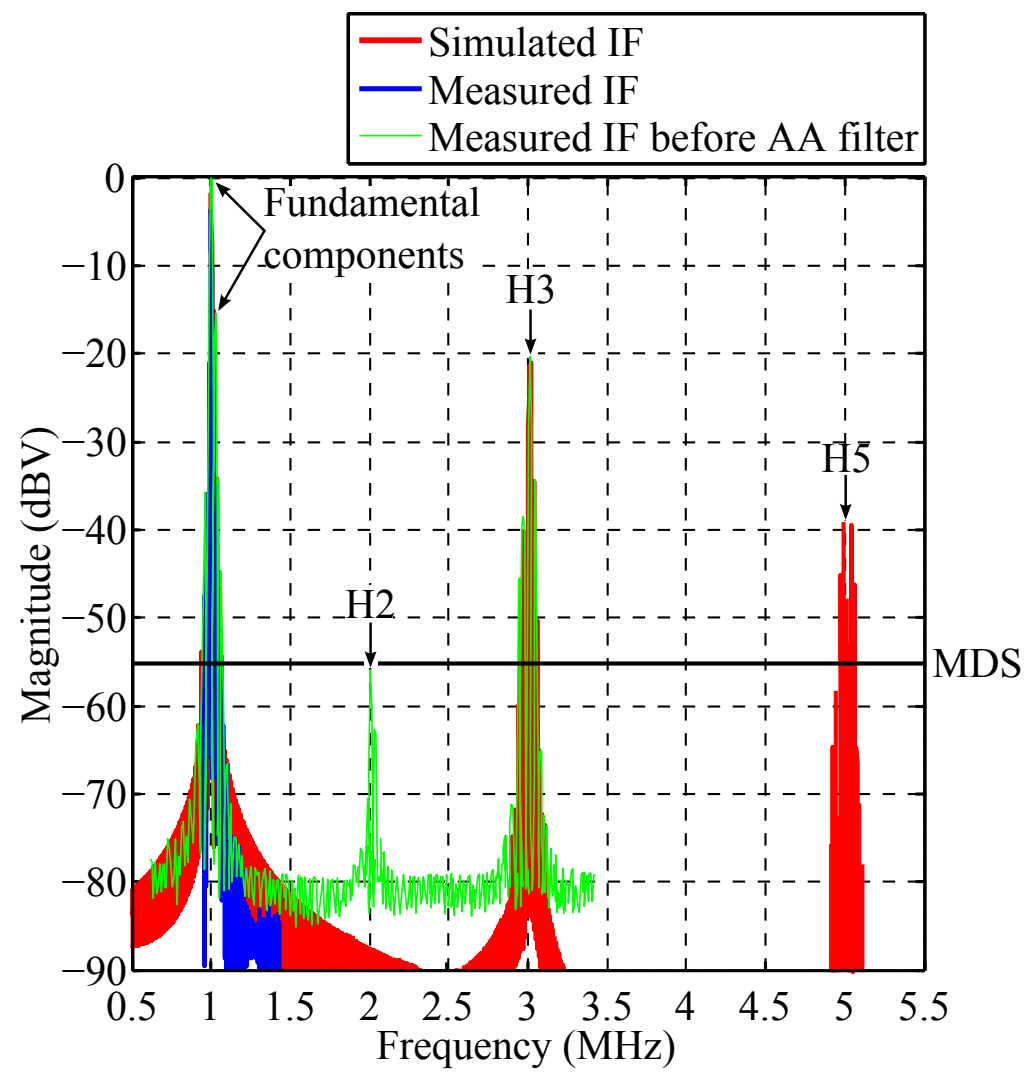

(a)

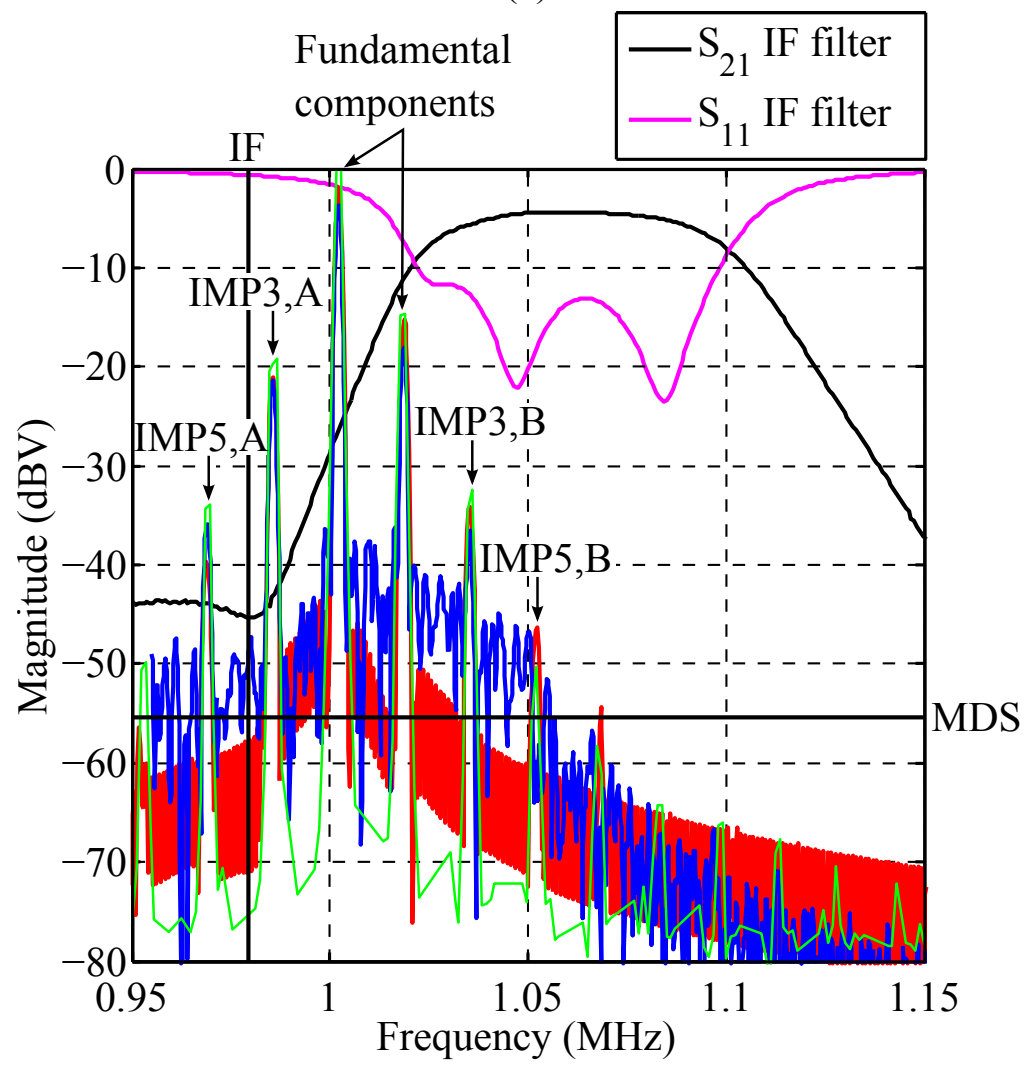

(b)

Figure 7.9: (a) The simulated and measured spectrum due to a two tone excitation $\left(P_{R x, 1}=-23 \mathrm{dBm}\right.$ and $\left.P_{R x, 2}=-48 \mathrm{dBm}\right)$. (b) Close-up spectral view of the measurements, simulation and IF filter response. 


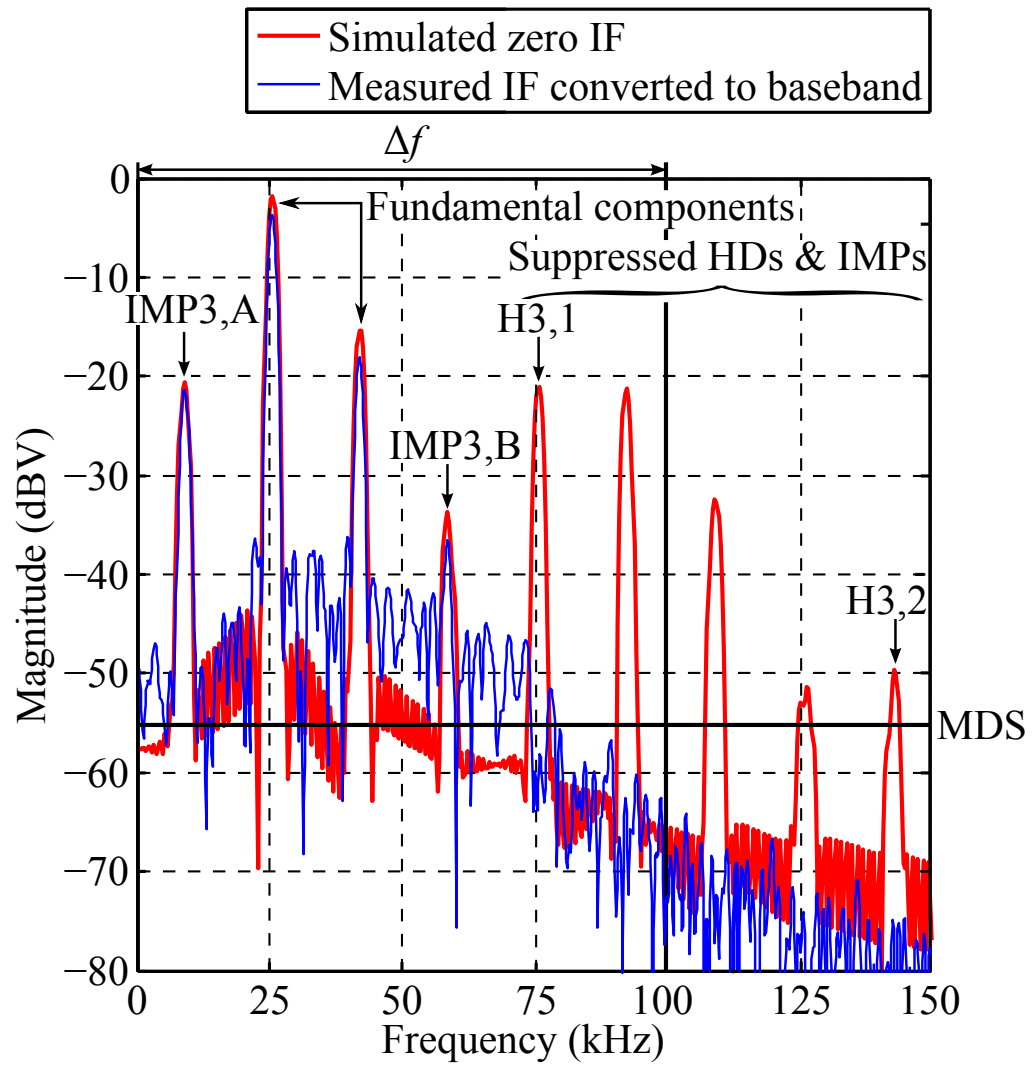

(a)

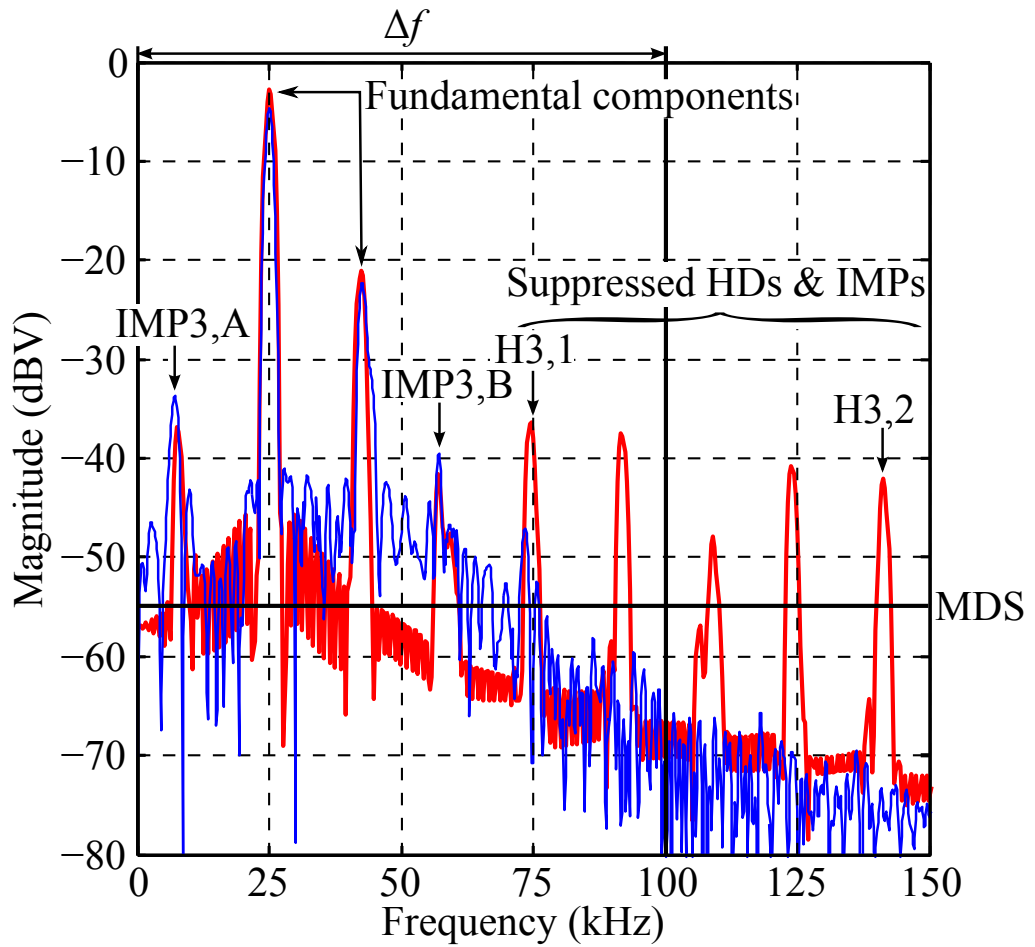

(b)

Figure 7.10: Two tone excitation with: (a) $P_{R x, 1}=-23 \mathrm{dBm}$ and $P_{R x, 2}=-48 \mathrm{dBm}$, (b) $P_{R x, 1}=-23 \mathrm{dBm}$ and $P_{R x, 2}=-58 \mathrm{dBm}$. Comparing the simulated spectrum for a zero-IF receiver to a measurement made using the IF receiver. The measured spectrum is transposed to baseband by subtracting the IF. 
Figure 7.10 (a) presents a baseband comparison between the simulated zero-IF receiver and a transposed measurement made using the IF receiver. All the harmonics and IMPs except IMP3,A and IMP3,B are suppressed by the IF receiver's anti-aliasing filter.

A second measurement is presented with $P_{R x, 1}=-23 \mathrm{dBm}$ and $P_{R x, 2}=-58 \mathrm{dBm}$, where the spectral locations of distortions are the same as in Fig. 7.9. The zero-IF and IF receivers are compared at baseband in Fig. 7.10 (b). Due to the lower saturation level the third order IMPs at $7 \mathrm{kHz}$ and $61 \mathrm{kHz}$ are lower than the same components in Fig. 7.10 (a). However, these IMPs are still detected as false targets by both the IF and zero-IF receivers.

When a large first reflection is received with much smaller subsequent reflections, a situation can arise where the low order IMPs are below the MDS level (see section 5.6.5). If the difference between the initial and subsequent reflections becomes so large that the subsequent reflection magnitudes become negligible, the best case scenario is achieved where a single large reflection remains that only generates harmonic distortions.

\subsection{Conclusion}

In this chapter, the noise figure is measured using the $\mathrm{Y}$ factor method. The measurement is presented in Fig. 7.4, where it is compared to the calculated result from Chapter 4. For the time domain interferometry measurements a controlled environment is created by using coaxial cables as the transmission medium. The measurements show that a zero-IF receiver is susceptible to harmonics and IMPs that an IF receiver can suppresses. However, both the IF and zero-IF receivers are susceptible to low order IMPs that fall in the receiver's passband. 


\section{Chapter 8}

\section{Conclusions and recommendations}

This study consists of the analysis, design and evaluation of an IF FMCW radar. The IF FMCW radar receiver is compared to an equivalent baseband (or zero-IF) receiver in the context of receiver noise, demodulator leakage, component non-linearity, and the problem of close range reflections.

\subsection{Research}

The objective of this study is to research the advantages to using an FMCW radar receiver with an IF offset. The IF topology is compared to a simulated baseband receiver implementation.

The radar design is outlined in Chapter 2, where the transmitter, IF offset LO, and receiver are discussed. Presented in Chapter 3 is a detailed description of the IF receiver design that includes a discussion on image rejection, signal quantization, anti-aliasing, and SFC. An IF filter (acting as SFC) is designed to suppress large reflections from targets close to the radar. If the receiver saturates, undesired harmonics and IMPs are generated. An anti-aliasing filter attenuates the out off band spurious signals. To understand the effect SFC has on the receiver's dynamic range a noise analysis is undergone in Chapter 4. The modelling and representation of non-linearities in receivers are discussed in Chapter 5 and the spurious free dynamic range is calculated. The LNA, demodulator and high voltage gain op-amps are investigated to determine which component is the limiting factor with regards to receiver saturation. A diode voltage limiter model is presented to represent the non-linear behaviour of a saturated op-amp. The amplitude of harmonics and IMPs are determined using an algebraic approximation of the non-linear model. This model is applied to predict under which conditions false echoes will be detected. The constructed IF receiver characteristics are measured in Chapter 6 and compared to calculations.

\subsection{Findings and recommendations}

From the measurements presented in Chapter 7, it is found that a large number of harmonics and IMPs generated by the IF receiver's non-linearity are suppressed. As discussed in Chapter 5 this application has limitations with regards to the received signal characteristics. However, it is clear that a zero-IF receiver is susceptible to undesired distortions an IF receiver can suppress. When more than one reflection is received and the receiver saturates, detectable low order IMPs could arise. The best case scenario is achieved when the difference between the initial and subsequent reflections becomes so large that the 
subsequent reflection magnitudes become negligible. Thus, resulting in a single large reflection that only generates harmonic distortions.

The dynamic range calculations in Chapter 5 show that the ADC dictates the receiver's effective dynamic range because it truncates the RF front-end's dynamic range.

The IQ demodulator is the main contributor to the receiver's noise figure. The noise performance of an IF receiver is potentially greater than an equivalent baseband receiver because pink noise (or $1 / f$ noise) reduces with an increase in operating frequency (see Chapter 4).

In Chapter 6 it is shown that the IF receiver is immune to the detection of false targets caused by a demodulator's LO to RF leakage.

For the radar design presented in this study, system integration and clock synchronisation between the DDS and MCU is recommended (see section 6.2.7).

The IF filter design presented in Chapter 3 decreases the receiver's sensitivity at low beat frequencies making the FMCW radar receiver less susceptible to close range targets that could cause receiver saturation.

Future work would include: integrating the system, designing antennas, investigating the non-linear noise characteristics of saturating components and the effect of gain compression on the receiver's dynamic range.

\subsection{Conclusion}

An FMCW radar receiver can saturate due to large reflections from close range targets. The study presents favourable results that show the IF receiver can suppress harmonics and IMPs a zero-IF receiver can not. However, some lower order IMPs still have the potential to fall within the IF receiver's band of interest. Depending on the nature of the excitation, the IF receiver can recover from different levels of saturation. Though the application has limitations with regards to the received signal characteristics, the IF receiver has better or equivalent performance when compared to a baseband receiver. 


\section{Appendix A}

\section{Receiver theory}

\section{A.1 Down conversion}

The process of down conversion is illustrated in Fig. A.1 by an idealized mixer that operates as a signal multiplier. The desired output component is the difference frequency term in Eq. (A.1.1) that it is selected by filtering the output signal $\left(v_{\text {out }}\right)$. The desired output frequency component is often referred to as the beat frequency, i.e. $f_{b}=f_{R F}-f_{L O}$ $[1]$.

$$
\begin{aligned}
v_{\text {out }}(t) & =v_{R F}(t) v_{L O}(t), \\
& =A_{R F} \cos \left(2 \pi t f_{R F}\right) A_{L O} \cos \left(2 \pi t f_{L O}\right), \\
& =\frac{A_{R F} A_{L O}}{2}\left\{\cos \left[2 \pi t\left(f_{R F}-f_{L O}\right)\right]+\cos \left[2 \pi t\left(f_{R F}+f_{L O}\right)\right]\right\} .
\end{aligned}
$$

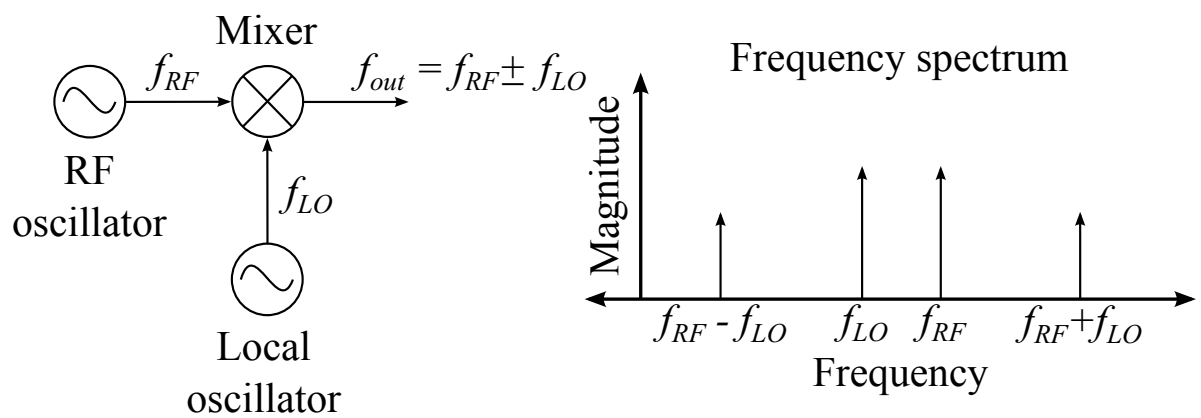

Figure A.1: The symbolic functional diagram of a signal multiplier (idealized mixer) illustrating frequency down conversion.

\section{A.2 Algebraic formula to determine the amplitudes of intermodulation products and harmonics caused by an arbitrary number of carriers}

The following mathematical expansion is described in [59] and [60] and referred to in section 5.6.3. 
As discussed in section 5.3 a non-linear device with a continue relationship between its input and output can be described by an infinite power series as follows

$$
v_{\text {out }}(t)=\sum_{k=0}^{\infty} a_{k} v_{\text {in }}(t)^{k}
$$

where $v_{\text {in }}(t)$ and $v_{\text {out }}(t)$ are respectively the input and output signals, and $a_{k}$ represents the coefficients of the power series [59].

To keep the equations succinct the explicitly written time dependencies are dropped, e.g. writing, $v_{i n}(t)$ as $v_{i n}$, etc. The input signal can consist of many cosine's with different amplitudes and frequencies as in Eq. (A.2.2),

$$
v_{\text {in }}=v_{1}+\cdots+v_{M},
$$

Therefore, Eq. (A.2.1) is rewritten as

$$
v_{\text {out }}=\sum_{k=0}^{\infty} a_{k}\left(\sum_{m=1}^{M} v_{m}\right)^{k} .
$$

The input signal is the sum of $M$ terms raised to an arbitrary power of $k$ and can be expressed as a multinomial expansion as follows

$$
v_{i n}^{k}=\left(\sum_{m=1}^{M} v_{m}\right)^{k}=\sum_{n_{1}, n_{2}, \cdots, n_{M}} \frac{k !}{n_{1} ! n_{2} ! \cdots n_{M} !} \prod_{i=1}^{M} v_{i}^{n_{i}},
$$

where the $i$-th cosine is $v_{i}=A_{i} \cos \theta_{i}$ and $\theta_{i}=\omega_{i}$. Equation (A.2.4) holds for positive integer values of $m$ and $k$. The sum is taken over all possible combinations of the integer indices $n_{1}$ through $n_{m}$ such that the sum of each combinations always adds up to $k$, thus

$$
k=n_{1}+n_{2}+\cdots+n_{M} .
$$

Note that the binomial formula is a specific case of the more general multinomial equation. In the binomial case the expansion of two terms is considered, thus $M=2$.

The product operator in Eq. (A.2.4) is expanded to

$$
\prod_{i=1}^{M} v_{i}^{n_{i}}=\left[\prod_{i=1}^{M} A_{i}^{n_{i}}\right]\left[\prod_{i=1}^{M}\left(\cos \theta_{i}\right)^{n_{i}}\right] .
$$

Applying Euler's identity to the factor containing the product of cosines in Eq. (A.2.6) results in

$$
\prod_{i=1}^{M}\left(\cos \theta_{i}\right)^{n_{i}}=\prod_{i=1}^{M}\left(\frac{e^{j \theta_{i}}+e^{-j \theta_{i}}}{2}\right)^{n_{i}} .
$$

The binomial expansion of Eq. (A.2.7) gives the following expression

$$
\prod_{i=1}^{M}\left(\frac{e^{j \theta_{i}}+e^{-j \theta_{i}}}{2}\right)^{n_{i}}=\prod_{i=1}^{M}\left(\frac{1}{2}\right)^{n_{i}} \sum_{k_{i}=0}^{n_{i}} \frac{n_{i} !}{k_{i} !\left(n_{i}-k_{i}\right) !} e^{j\left(2 k_{i}-n_{i}\right) \theta_{i}} .
$$

Equation (A.2.8) can be rearranged into independent factors to allow for easier manipulation. It follows from Eq. (A.2.5) and

$$
\prod_{i=1}^{M}\left(\frac{1}{2}\right)^{n_{i}}=\frac{1}{2^{k}}
$$


that the power product of cosines described by Eq. (A.2.8) can be expressed as

$$
\begin{aligned}
\prod_{i=1}^{M}\left(\cos \theta_{i}\right)^{n_{i}}= & \frac{1}{2^{k}} \sum_{k_{1}=0}^{n_{1}} \cdots \sum_{k_{M}=0}^{n_{M}}\left[\prod_{p=1}^{M} \frac{n_{p} !}{k_{p} !\left(n_{p}-k_{p}\right) !}\right] \\
& \cdot \exp \left[j \sum_{i=1}^{M}\left(2 k_{i}-n_{i}\right) \theta_{i}\right]
\end{aligned}
$$

Note that $\prod_{p=1}^{M} n_{p} !=n_{1} ! n_{2} ! \cdots n_{m}$ ! then the sum of input signals $v_{i n}^{k}$ is written in its expanded form by plugging Eqs. (A.2.6) and (A.2.10) into Eq. (A.2.4) resulting in Eq. (A.2.11).

$$
\begin{aligned}
v_{i n}^{k}= & \sum_{n_{1}, n_{2}, \cdots, n_{M}} \frac{k !}{2^{k}}\left[\prod_{i=1}^{M} A_{i}^{n_{i}}\right] \\
& \cdot \sum_{k_{1}=0}^{n_{1}} \cdots \sum_{k_{M}=0}^{n_{M}}\left[\prod_{p=1}^{M} \frac{1}{k_{p} !\left(n_{p}-k_{p}\right) !}\right] \\
& \cdot \exp \left[j \sum_{i=1}^{M}\left(2 k_{i}-n_{i}\right) \theta_{i}\right]
\end{aligned}
$$

From Eq. (A.2.11) it is clear that all the possible combinations of input carriers involve frequencies of the form $\theta=\alpha_{1} \theta_{1}+\alpha_{2} \theta_{2}+\cdots+\alpha_{M} \theta_{M}$, where each $\alpha_{i}$ is an integer.

From Eq. (A.2.11) a formula is determined that gives the amplitude $V$ for a particular IMP at frequency $\theta$ that is selected by assigning values to $\alpha_{1}, \alpha_{2}, \cdots, \alpha_{M}$. Applying Euler's identity cosines are formed from Eq. (A.2.11) by allowing terms with $\alpha_{i}=2 k_{i}-n_{i}$ and $\alpha_{i}=-\left(2 k_{i}-n_{i}\right)$ to contribute to the chosen intermodulation component for all possible values of $i$. Let $V_{k}$ * denote the part of Eq. (A.2.11) that remains when only these two sets of $k_{i}$ 's are used, a factor two is included as part the Euler identity for nonzero frequencies, $\theta \neq 0$ then $\varepsilon_{N}=2$ [59]. Thus a specific cosine term $V_{k} *=V_{k} \cos \left(\alpha_{1} \theta_{1}+\right.$ $\left.\alpha_{2} \theta_{2}+\cdots+\alpha_{M} \theta_{M}\right)$ is selected from all the combinations generated by the $k$-th term in the power series expansion of Eq. (A.2.3) where

$$
V_{k}=\varepsilon_{N} \sum_{n_{1}, n_{2}, \cdots, n_{M}} \frac{k !\left[A_{1}^{n_{1}} \cdots A_{M}^{n_{M}}\right]}{2^{k}}\left[\prod_{p=1}^{M} \frac{1}{\left(\frac{n_{p}+\alpha_{p}}{2}\right) !\left(\frac{n_{p}-\alpha_{p}}{2}\right) !}\right] .
$$

For the DC component at $\theta=0$ when $\varepsilon_{N}=1$ all values of $\alpha$ are zero and only one set of $k_{i}$ 's contribute to $V_{k} *$.

The only terms in Eq. (A.2.12) that contribute to the chosen IMP are those for which $n_{i}$ are expressed as $n_{i}=\left|\alpha_{i}\right|+2 q_{i}$, where $q_{i}$ is a positive integer

$$
q_{i}=\frac{n_{i}+\left|\alpha_{i}\right|}{2}
$$

The order of the selected IMP is defined as

$$
N=\left|\alpha_{1}\right|+\left|\alpha_{2}\right|+\cdots+\left|\alpha_{M}\right|,
$$

because the component's spectral location is related to the fundamental input carriers that contribute to its amplitude. Therefore Eq. (A.2.12) and its summation rule is redefined 
to only include the contributing terms resulting in, $q_{1}+q_{2}+\cdots+q_{M}=\frac{1}{2}(k-N)$, and $V_{k}$ as

$$
V_{k}=\varepsilon_{N} \sum_{q_{1}, q_{2}, \cdots, q_{M}} \frac{k !\left[A_{1}^{\left|\alpha_{1}\right|+2 q_{1}} \cdots A_{M}^{\left|\alpha_{M}\right|+2 q_{M}}\right]}{2^{k}}\left[\prod_{p=1}^{M} \frac{1}{q_{p} !\left(\left|\alpha_{p}\right|+q_{p}\right) !}\right] .
$$

From the newly defined summation rule, $\sum_{q_{1}, q_{2}, \cdots, q_{M}}$, it is seen that $V_{k}$ is zero if, $k-N$ is not an even positive integer. This is because it is impossible to sum over a fractional or negative number of terms. Therefore IMPs of order $N$ is produced by terms in Eq. (A.2.1) for which the degree $k$ is greater than or equal to $N$. Also, the value of $k$ must be odd or even as $N$ is odd or even [59]. With this knowledge the total intermodulation amplitude can be determined by

$$
V=a_{N} V_{N}+a_{N+2} V_{N+2}+a_{N+4} V_{N+4}+\cdots .
$$

Each contributing term is included in Eq. (A.2.16) and their amplitudes are calculated using Eq. (A.2.15), thus an IMP's total amplitude is determined by

$$
\begin{aligned}
V & =\varepsilon_{N} \sum_{L=0}^{\infty} \frac{a_{N+2 L}(N+2 L) !}{2^{(N+2 L)}} \sum_{q_{1}, q_{2}, \cdots, q_{M}} \prod_{p=1}^{M} \frac{A_{p}^{\left|\alpha_{p}\right|+2 q_{p}}}{q_{p} !\left(\left|\alpha_{p}\right|+q_{p}\right) !}, \\
& =\varepsilon_{N}\left[\prod_{p=1}^{M} A_{p}^{\left|\alpha_{p}\right|}\right] \sum_{L=0}^{\infty} \frac{a_{N+2 L}(N+2 L) !}{2^{(N+2 L)}} \Phi(M, L) .
\end{aligned}
$$

In Eq. (A.2.17) the multiple summation is over all non-negative integers $q_{p}$ such that $q_{1}+q_{2}+\cdots+q_{M}=L$,

$$
\Phi(M, L)=\underbrace{\sum_{q_{1}, q_{2}, \cdots, q_{M}}}_{q_{1}+q_{2}+\cdots+q_{M}=L} \prod_{p=1}^{M} \frac{A_{p}^{2 q_{p}}}{q_{p} !\left(\left|\alpha_{p}\right|+q_{p}\right) !} .
$$

The summation in Eq. (A.2.18) can be represented as

$$
\sum_{q_{1}, q_{2}, \cdots, q_{M}}=\sum_{q_{M}=0}^{L} \sum_{q_{M-1}=0}^{L_{M-1}} \cdots \sum_{q_{i}=0}^{L_{i}} \cdots \sum_{q_{2}=0}^{L_{2}},
$$

where $L_{i}=L-q_{M}-q_{M-1}-\cdots-q_{i+1}$ for $i=1,2, \cdots, M-1$ and $q_{1}=L_{1}$.

To solve for Eq. (A.2.18) a iterative method derived by Sea and Vacroux in [60] is used. The method is based on the well-known technique whereby $a_{0}+a_{1} x+a_{2} x^{2}+\cdots+a_{K} x^{K}$ can be evaluated recursively by a computer as $a_{0}+x\left(a_{1}+x\left(a_{2}+\cdots+x\left(a_{K-1}+x a_{K}\right) \cdots\right)\right)$. Equation (A.2.18) is redefined in a recursive format as

$$
\Phi(r, q)=\sum_{q_{r}=0}^{q} \frac{A_{r}^{2 q_{r}}}{q_{r} !\left(\left|\alpha_{r}\right|+q_{r}\right) !} \Phi\left(r-1, q-q_{r}\right), \quad r=2, \cdots, M,
$$

where the first iteration is calculated as follows

$$
\Phi(1, q)=\frac{A_{1}^{2 q}}{q !\left(\left|\alpha_{1}\right|+q\right) !} .
$$

If $V$ is calculated by Eq. (A.2.17) and a truncated power series representation of Eq. (A.2.1) is used, $L$ has a maximum value represented by $L_{0}$. Thus $\Phi(M, L)$ is computed 
for $L=0,1, \cdots, L_{0}$, where $L_{0}=\frac{k_{0}-N}{2}$ and $k_{0}$ is equal to the order of the highest order polynomial in the truncated power series. The recursive calculation is implemented in MATLAB by first computing $\Phi(1, q)$ for $q=0,1, \cdots, L_{0}$ using Eq. (A.2.21). Then applying Eq. (A.2.20) recursively where $r=2,3, \cdots, M$ and $\Phi(r, q)$ is computed for the truncated $q$, i.e. $q=0,1, \cdots, L_{0}$. 


\section{Appendix B}

\section{Matlab code}

\section{B.1 Synthesis of a general Chebyshev filter function}

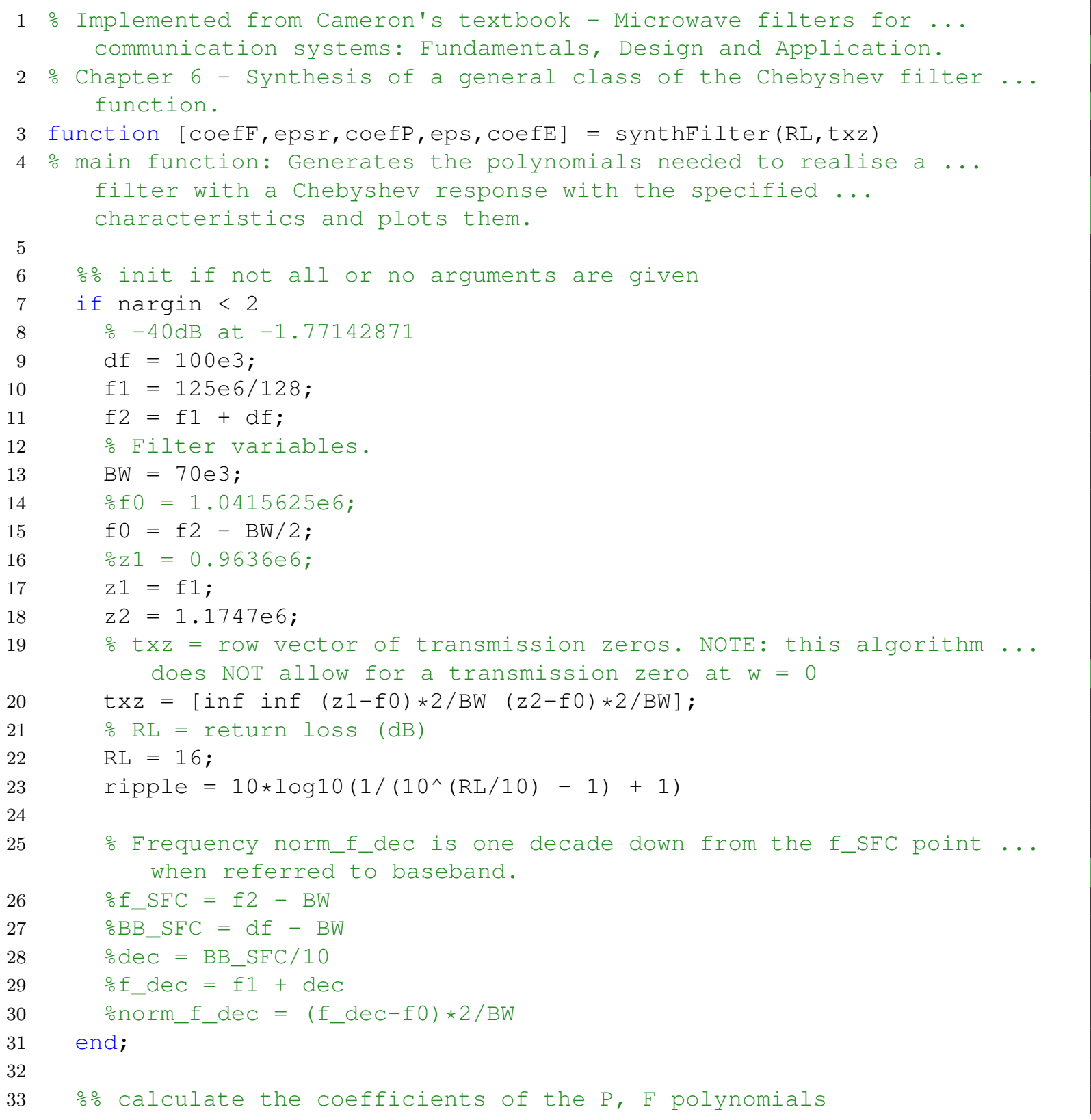




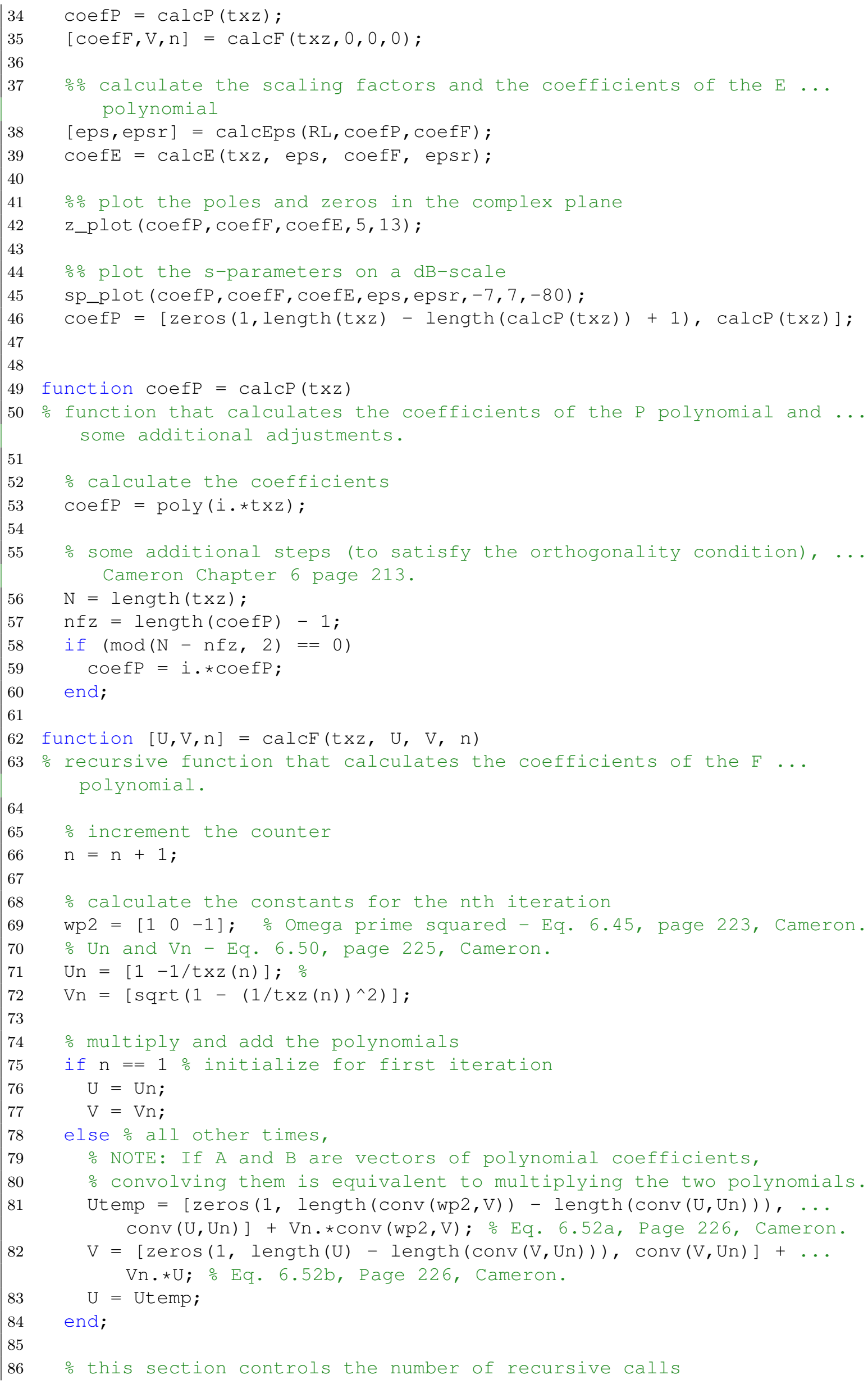




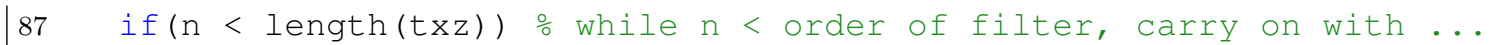

88

89

90

91

92

93

94 function [eps, epsr] = calcEps (RL, coefP, coefF)

95

96

97

98

99

100

101

102

103

104

105

106

107

108 
$134 \operatorname{plot}(\operatorname{real}(\operatorname{roots}(\operatorname{coefF})), \operatorname{imag}(\operatorname{roots}(\operatorname{coefF})), \operatorname{crx})$;

135 plot (real(roots (coefP)), imag(roots (coefP)), 'bo');

136 hold off;

137 warning off;

138 legend('Roots of E','Roots of F', 'Roots of P');

139 warning on;

140 otitle('The roots of the E, F and $P$ polynomials');

141 ylabel('Im');

142 xlabel ('Re');

143 grid on;

144 axis([-Re_range/2 Re_range/2 -Im_range/2 Im_range/2]);

145

146 function sp_plot (coefP, coefF, coefE, eps, epsr,min_w, max_w, min_dB)

47 function that plots S11 and S21 on a dB-scale

148

149 o calculate s11 and s21

$150 \quad \mathrm{~W}=$ min_w: $\left(\max \_w-m i n \_w\right) / 20000: \max \_w$;

$151 \mathrm{~s} 11=$ polyval $(\operatorname{coefF}, i \cdot \star w) \cdot /$ epsr./polyval (coefE, i.*w);

152 s21 = polyval (coefp, i.*w) ./eps./polyval (coefE, i.*w);

153

154 \% plot the parameters

$155 \mathrm{fig} 2=$ figure (21);

156 clf(fig2,'reset');

157 hold on;

158 otitle('The magnitudes of S_\{11\} and S_\{21\} versus frequency');

159 ylabel ('|S| [dB]') ;

$160 \mathrm{xlabel}$ ('\omega $[\mathrm{rad} / \mathrm{s}]$ ') ;

161 warning off;

$162 \operatorname{plot}(w, 20 * \log 10(\operatorname{abs}(\mathrm{s} 11)), \quad$ 'r');

$163 \operatorname{plot}\left(\mathrm{w}, 20 * \log 10(\mathrm{abs}(\mathrm{s} 21)), \mathrm{b}^{\prime}\right)$;

164 oplot (norm_f_dec, $20 * \log 10(\operatorname{abs}(\mathrm{s} 21))$, ' g')

165 warning on;

166 legend ('S_\{11\}', 'S_ $\{21\}$ ');

167 hold off;

168 grid on;

169 axis ([min_w max_w min_dB 0 ]);

\section{B.2 Synthesis of the fully-canonical coupling matrix}

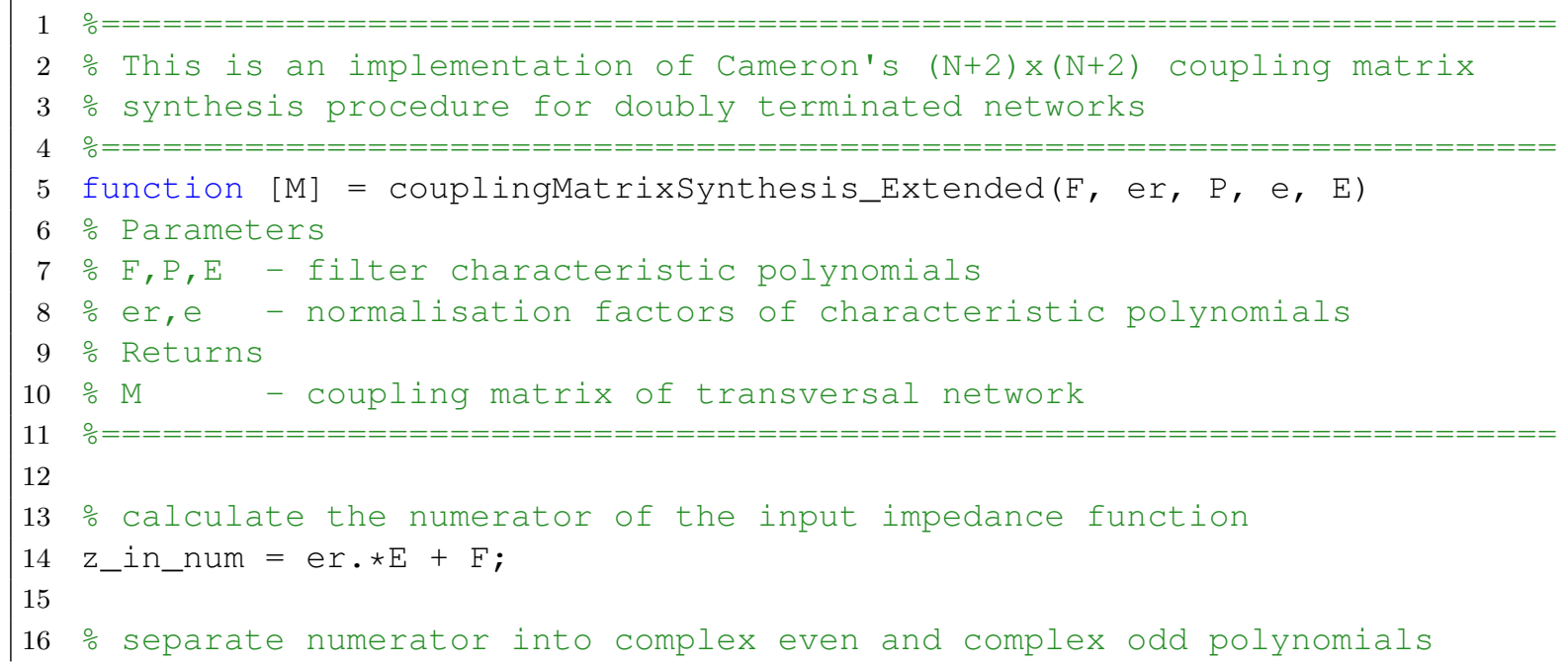




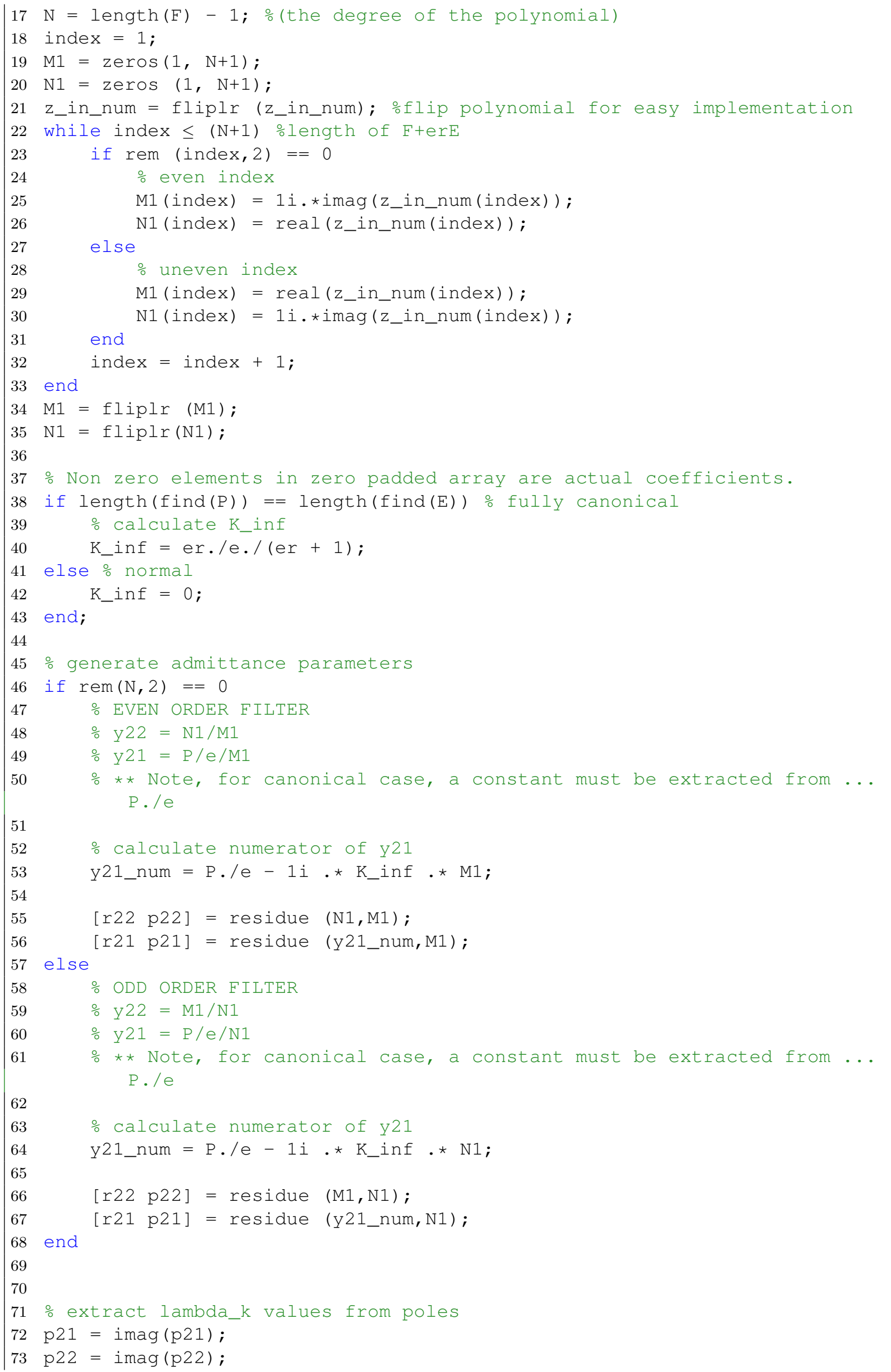




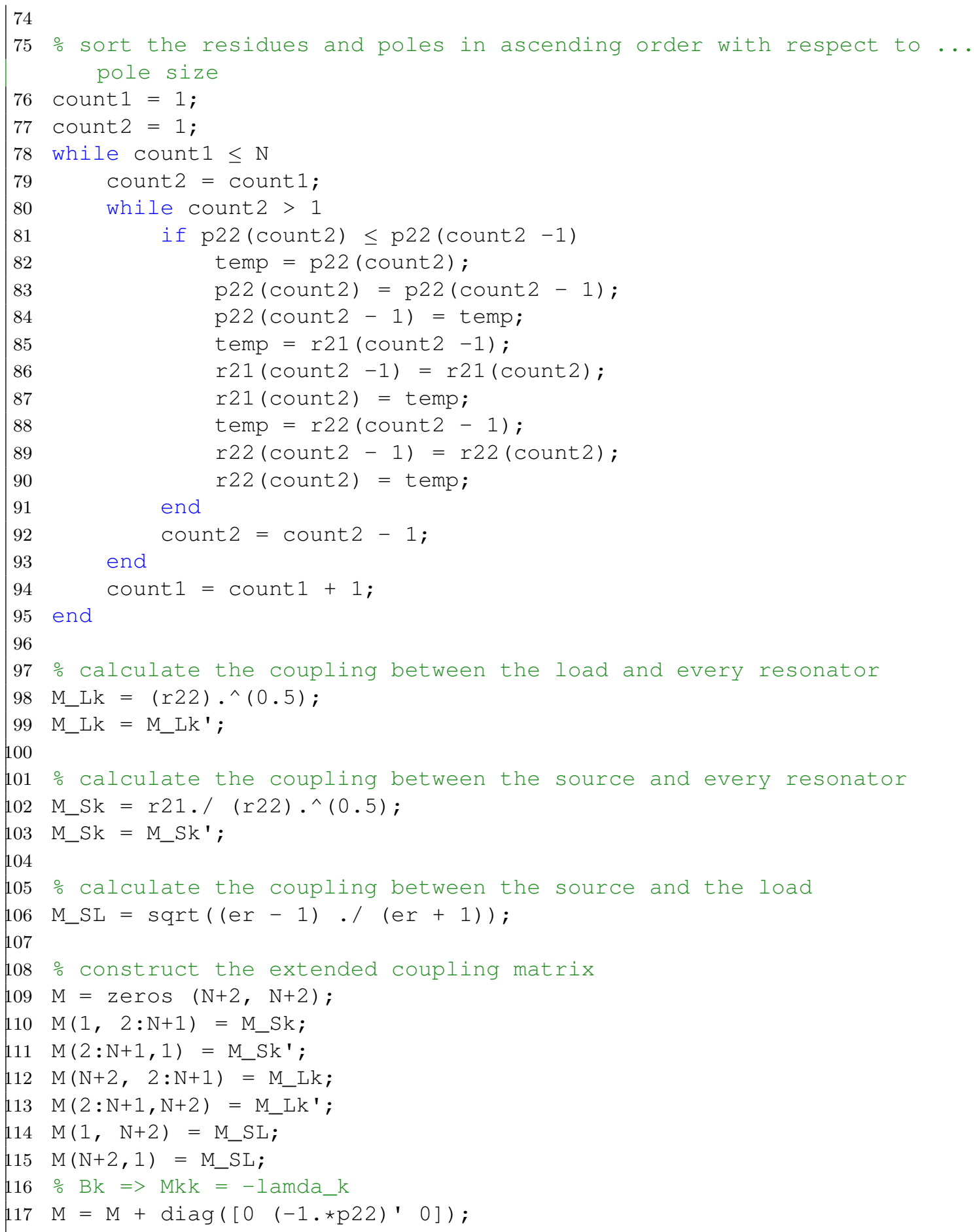

\section{B.3 Similarity transformation and matrix reconfiguration}

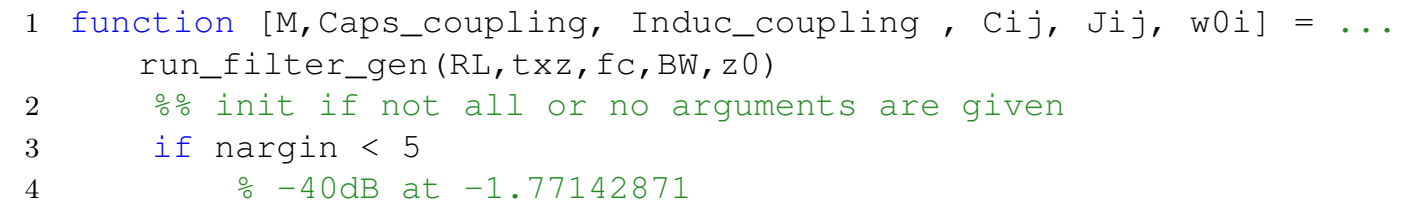




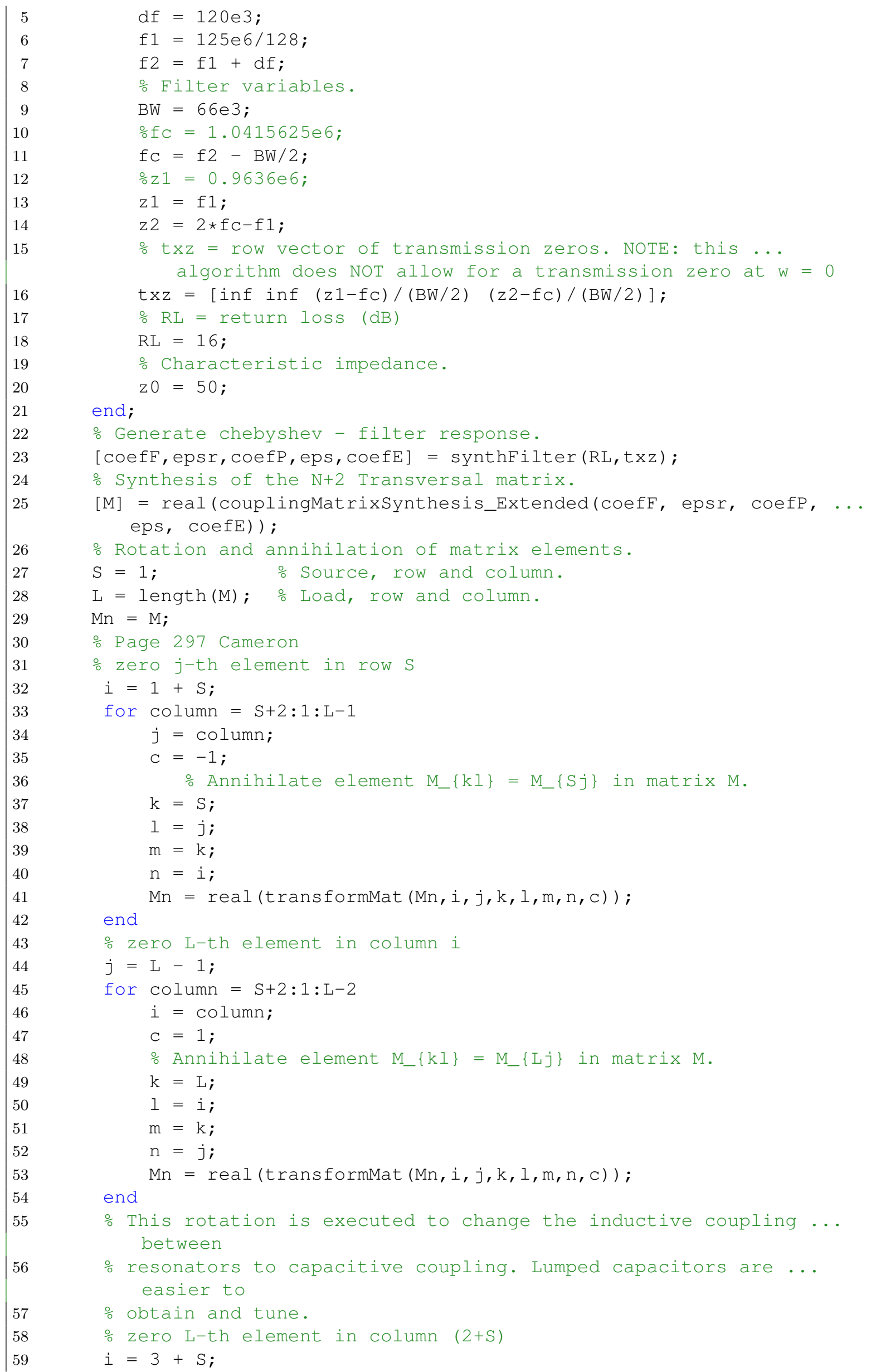




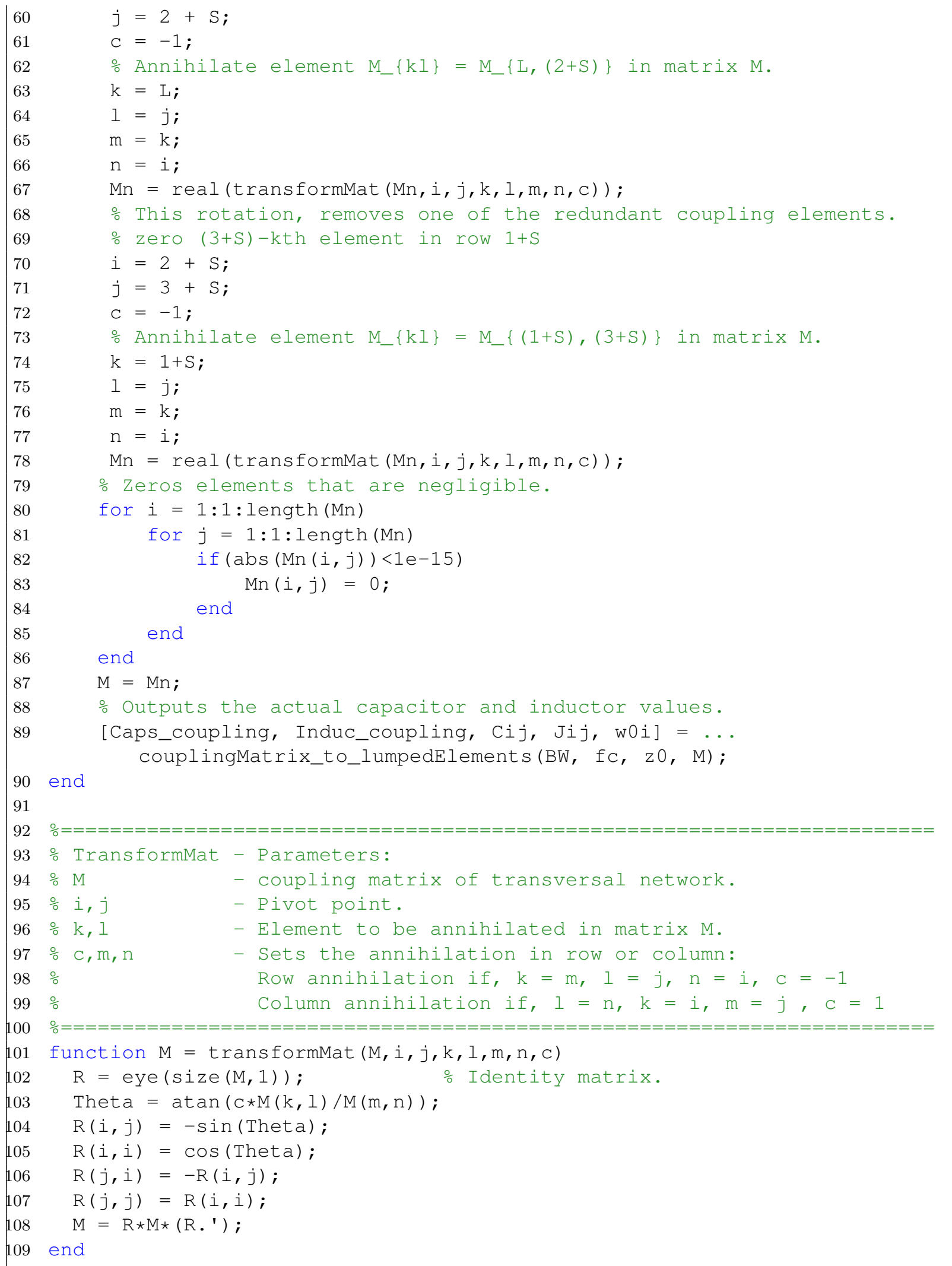




\section{B.4 Coupling matrix to lumped elements}

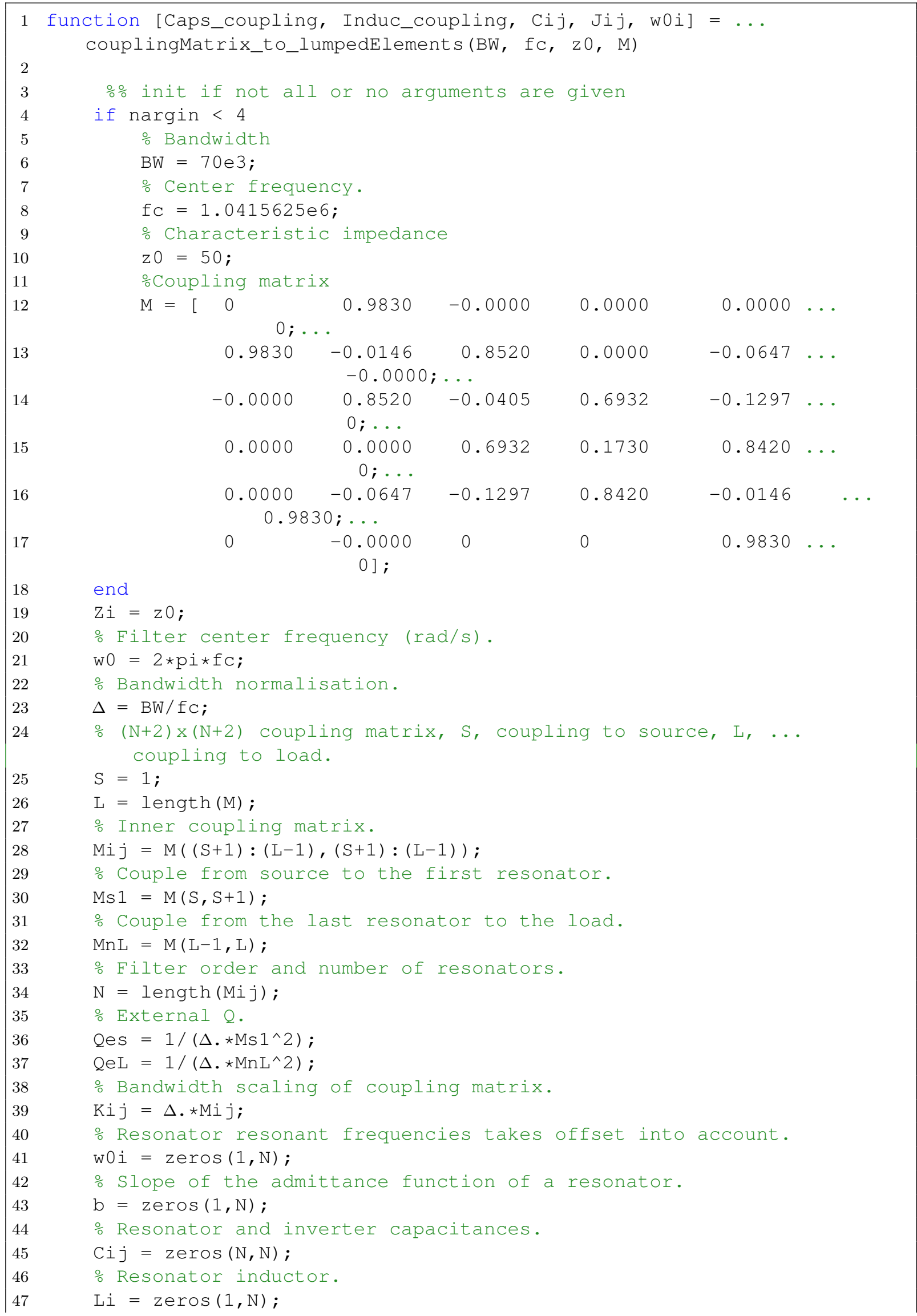




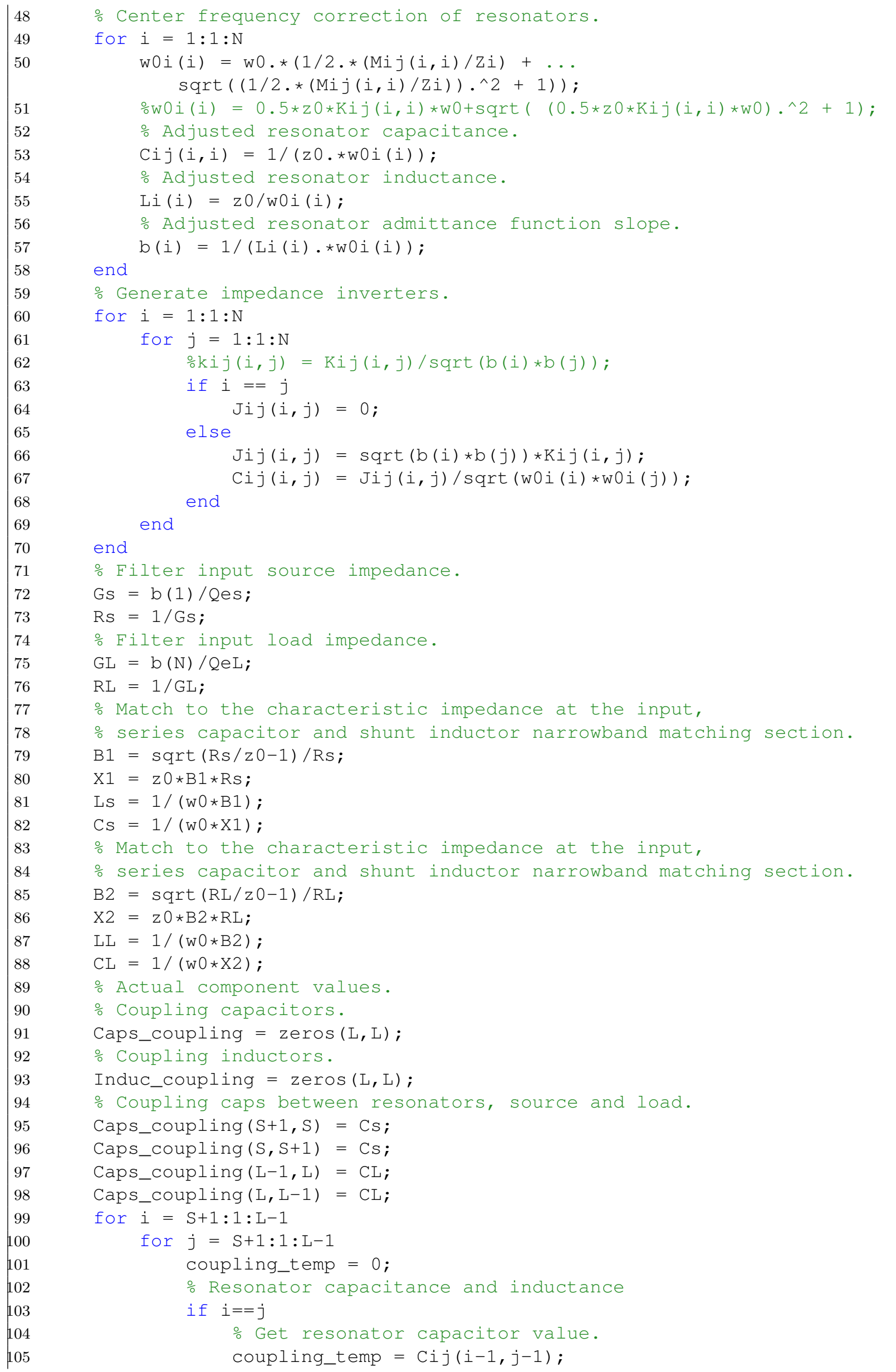




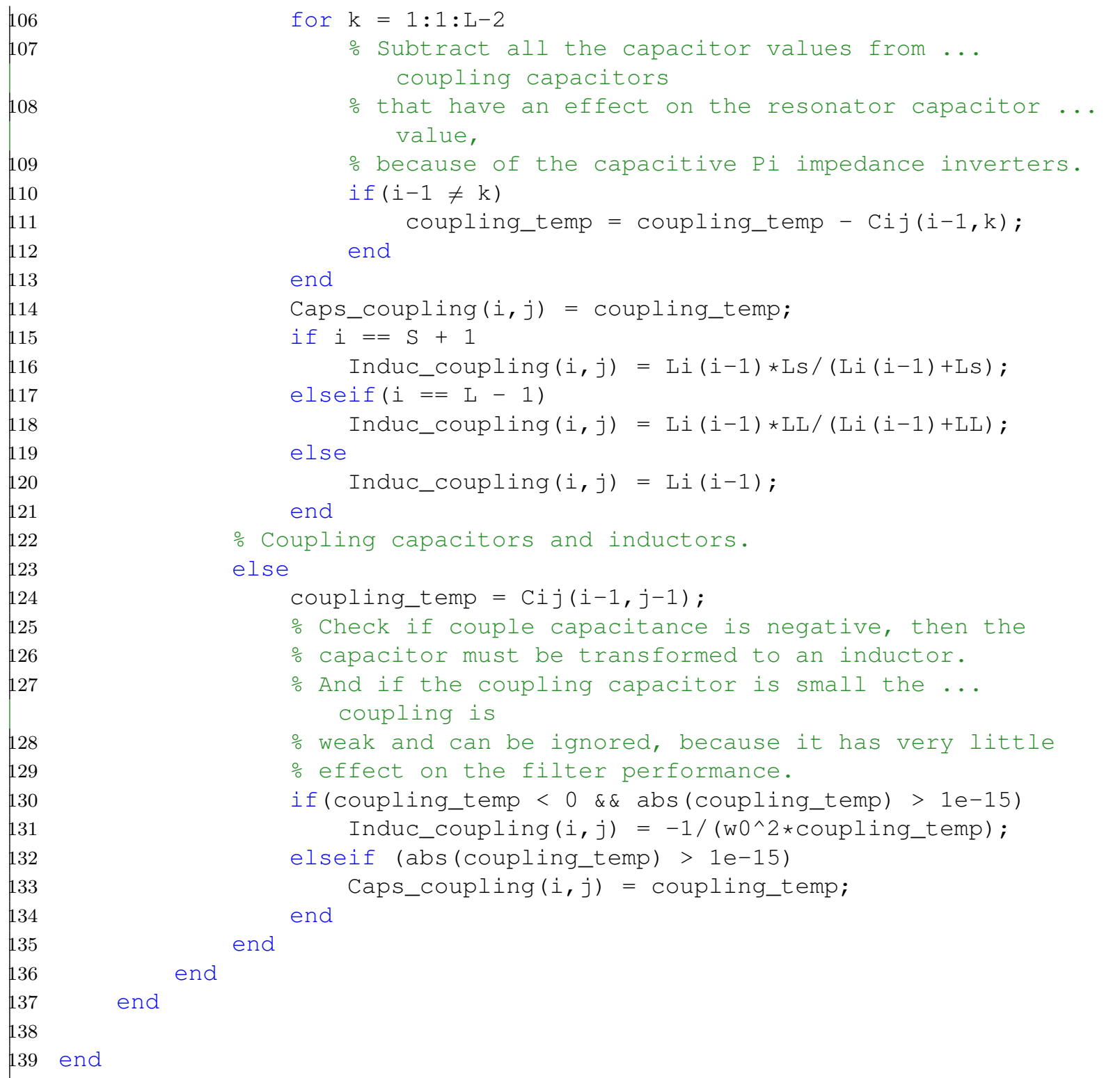

\section{B.5 Noise figure and Dynamic range calculation code}

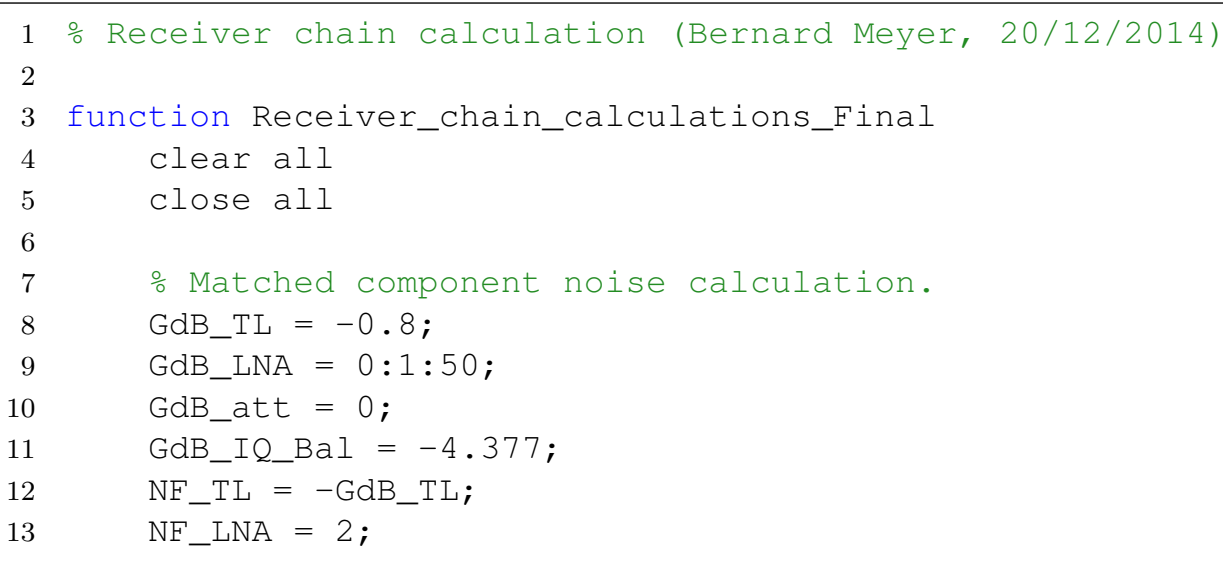




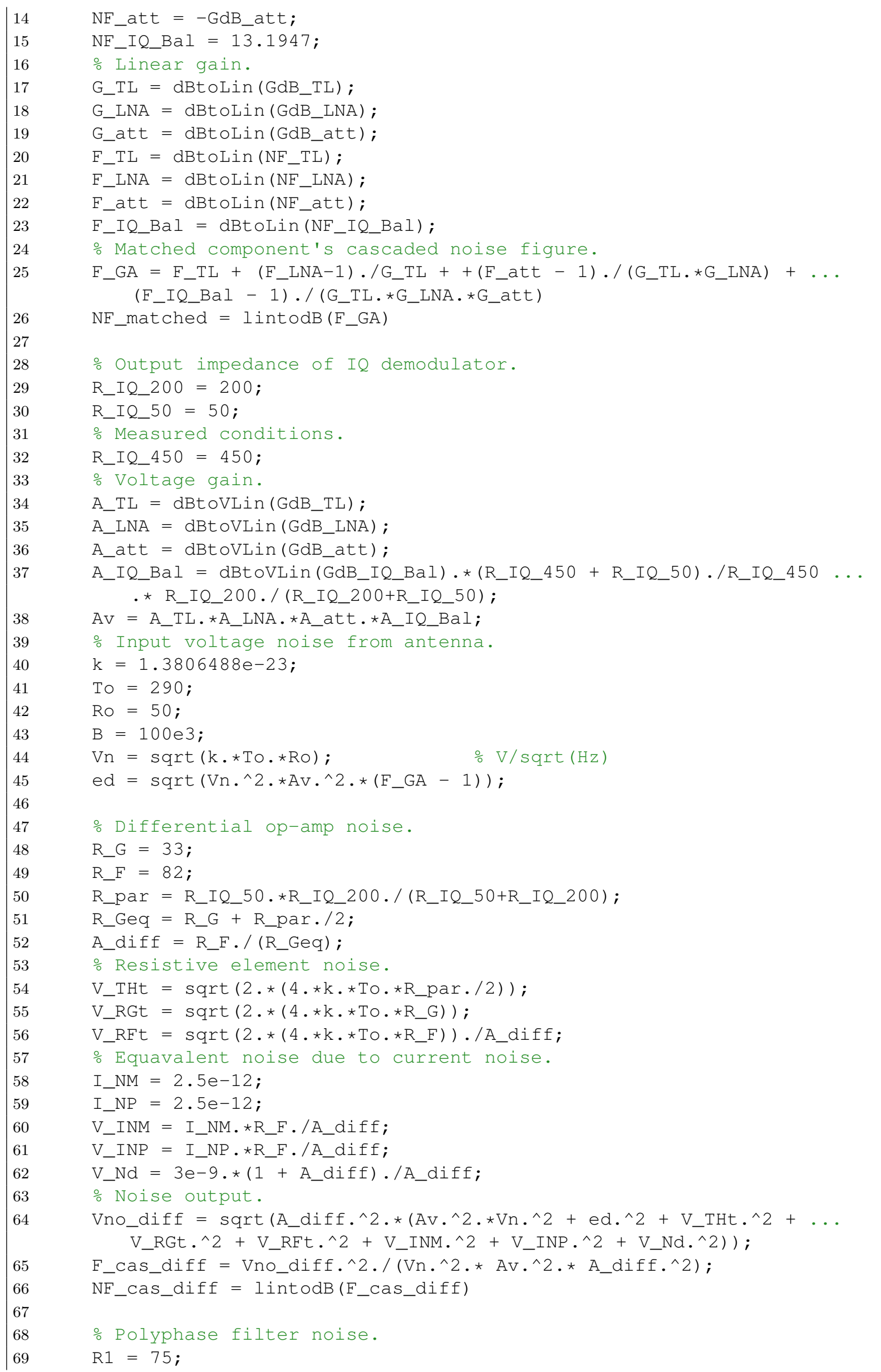




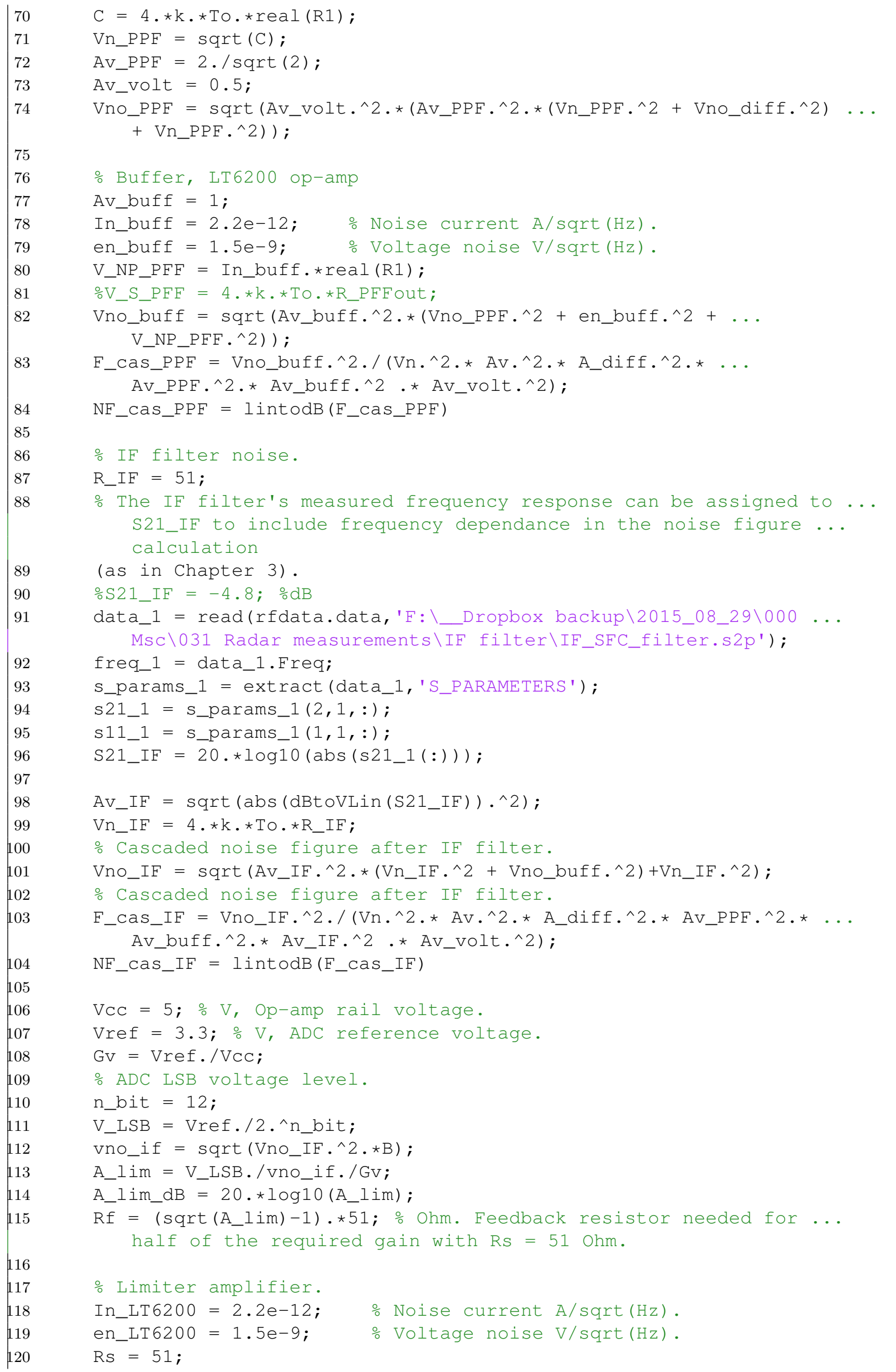




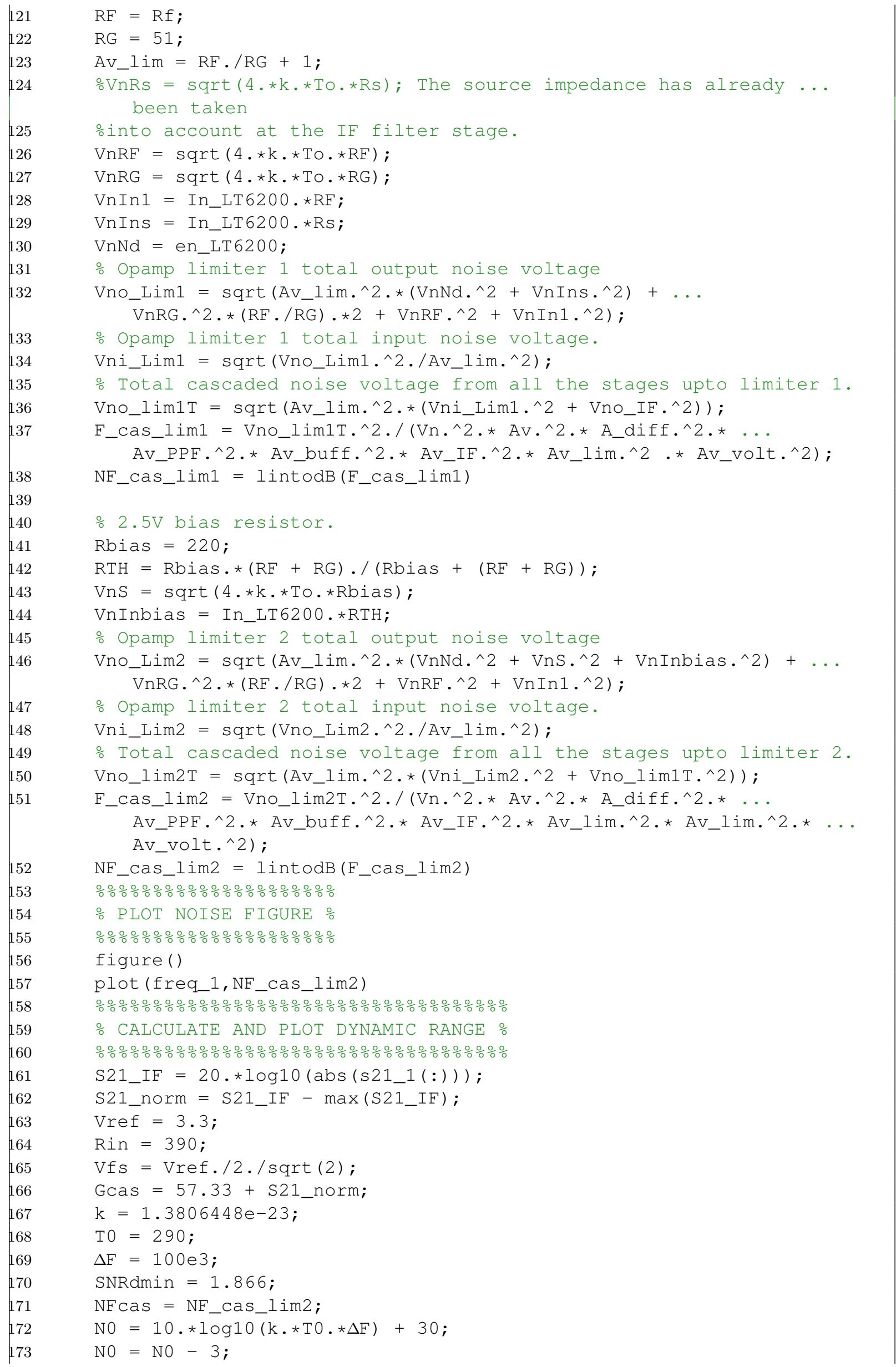




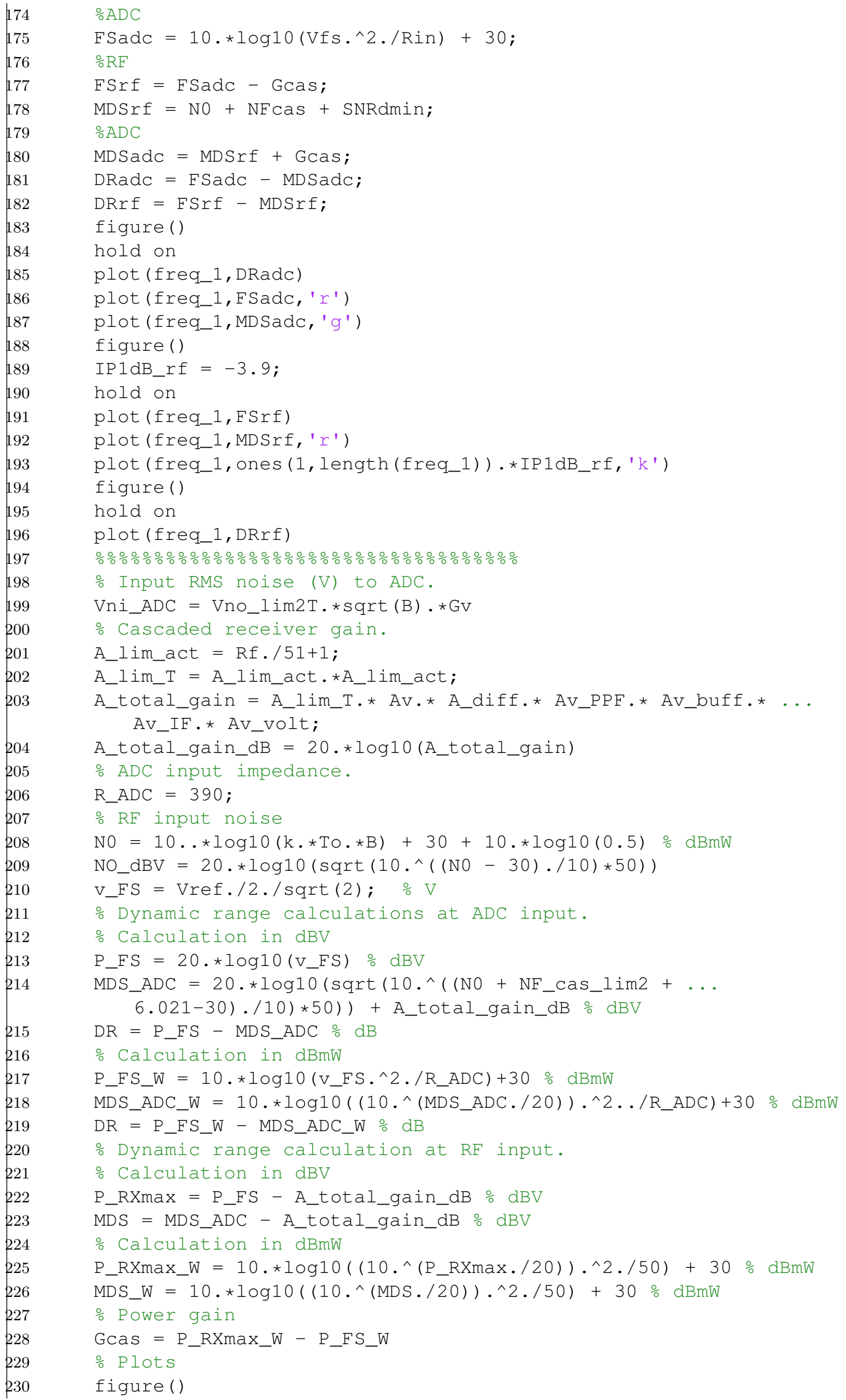




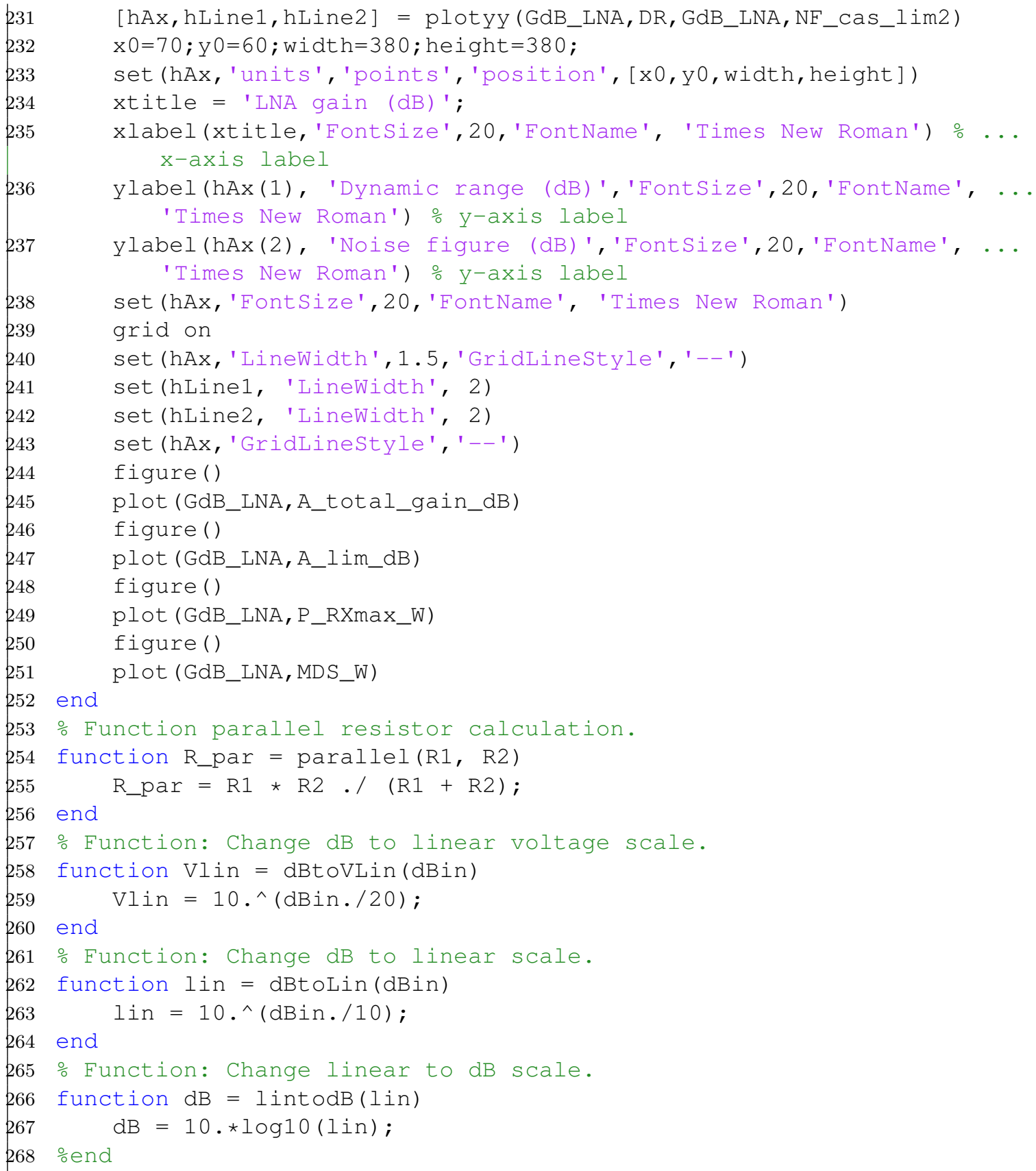

\section{B.6 LT6200 non-linear simulation code}

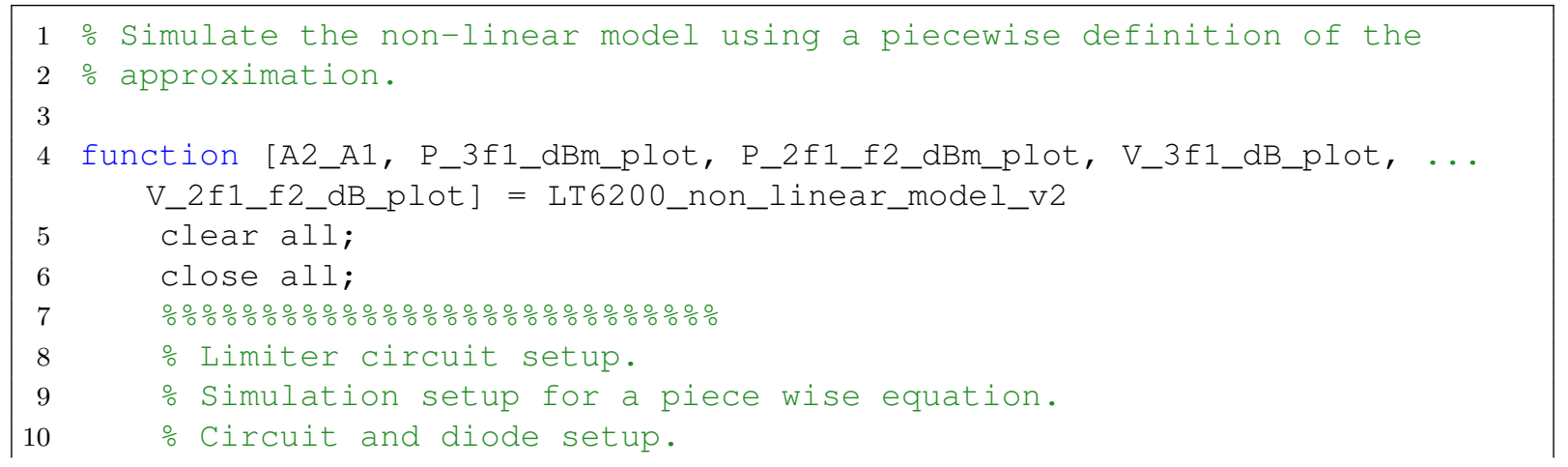




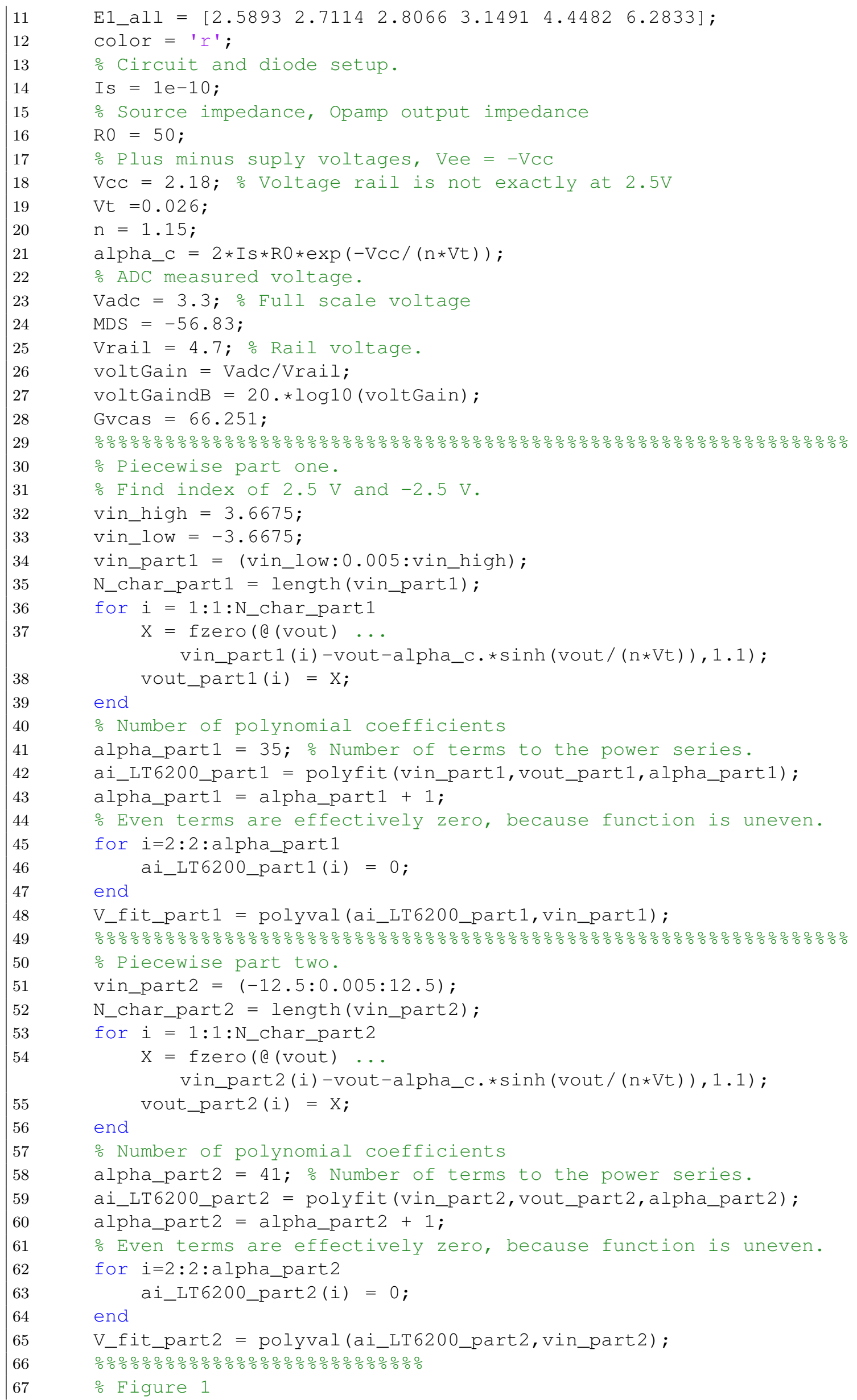




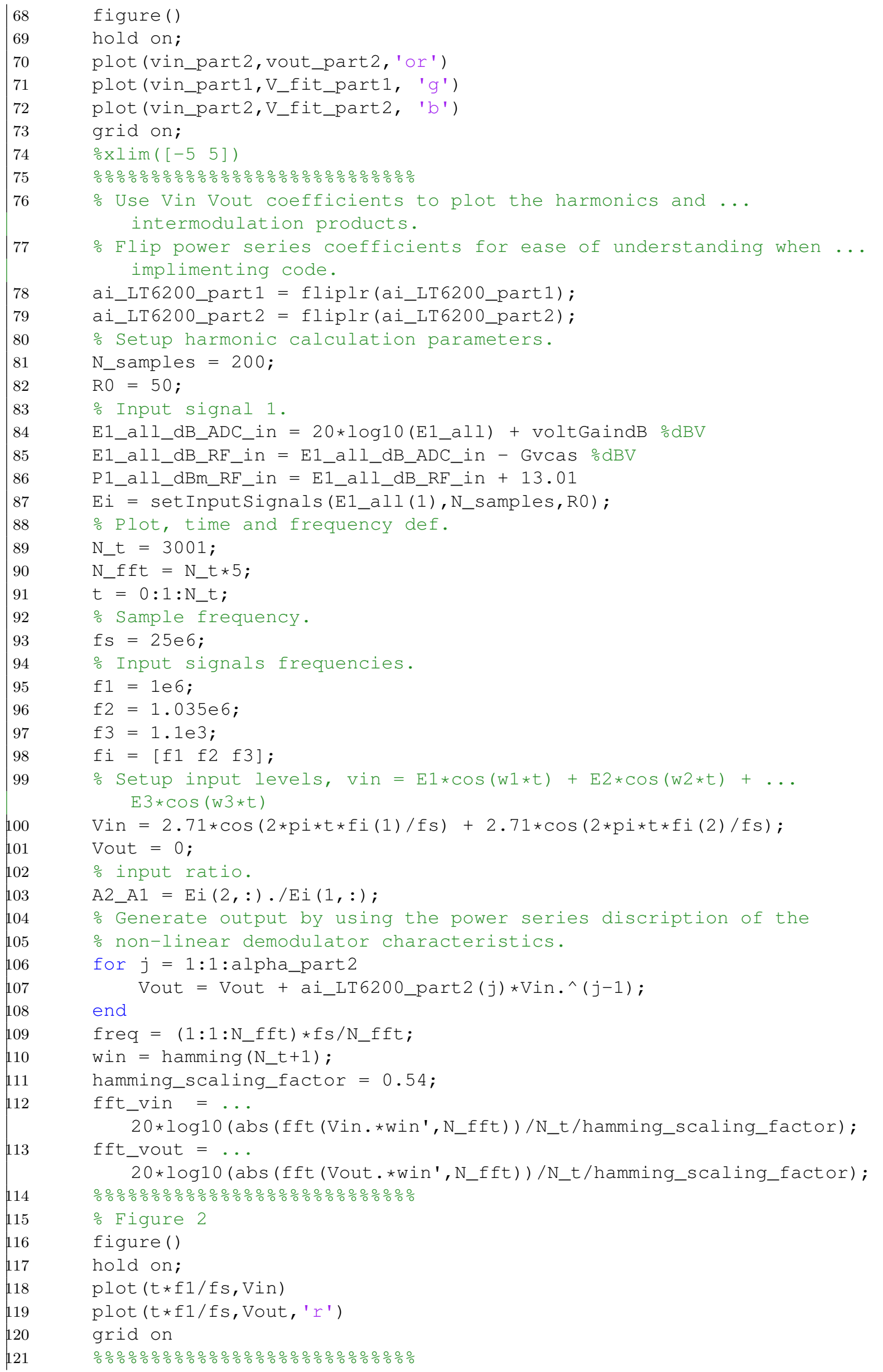




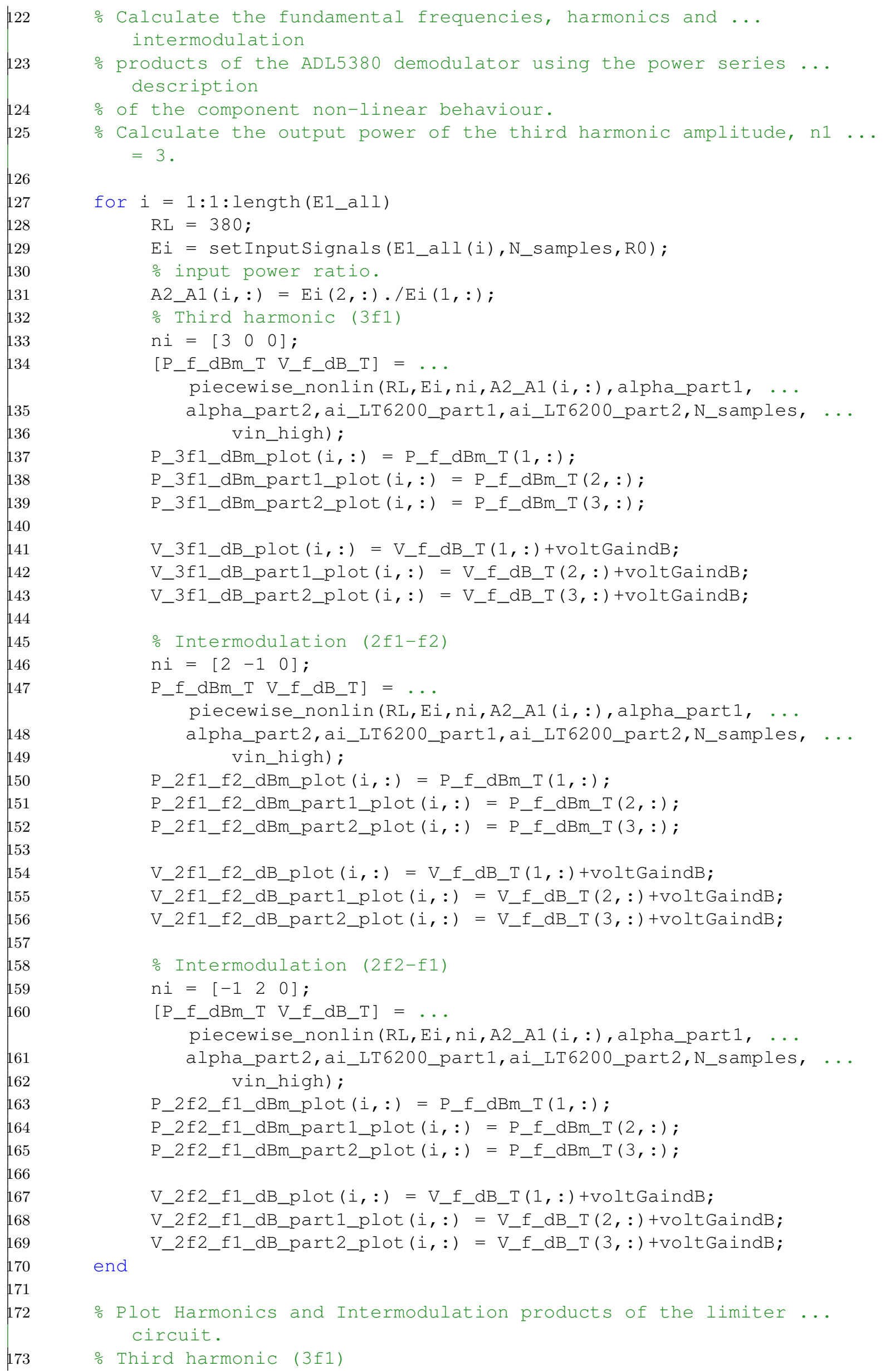




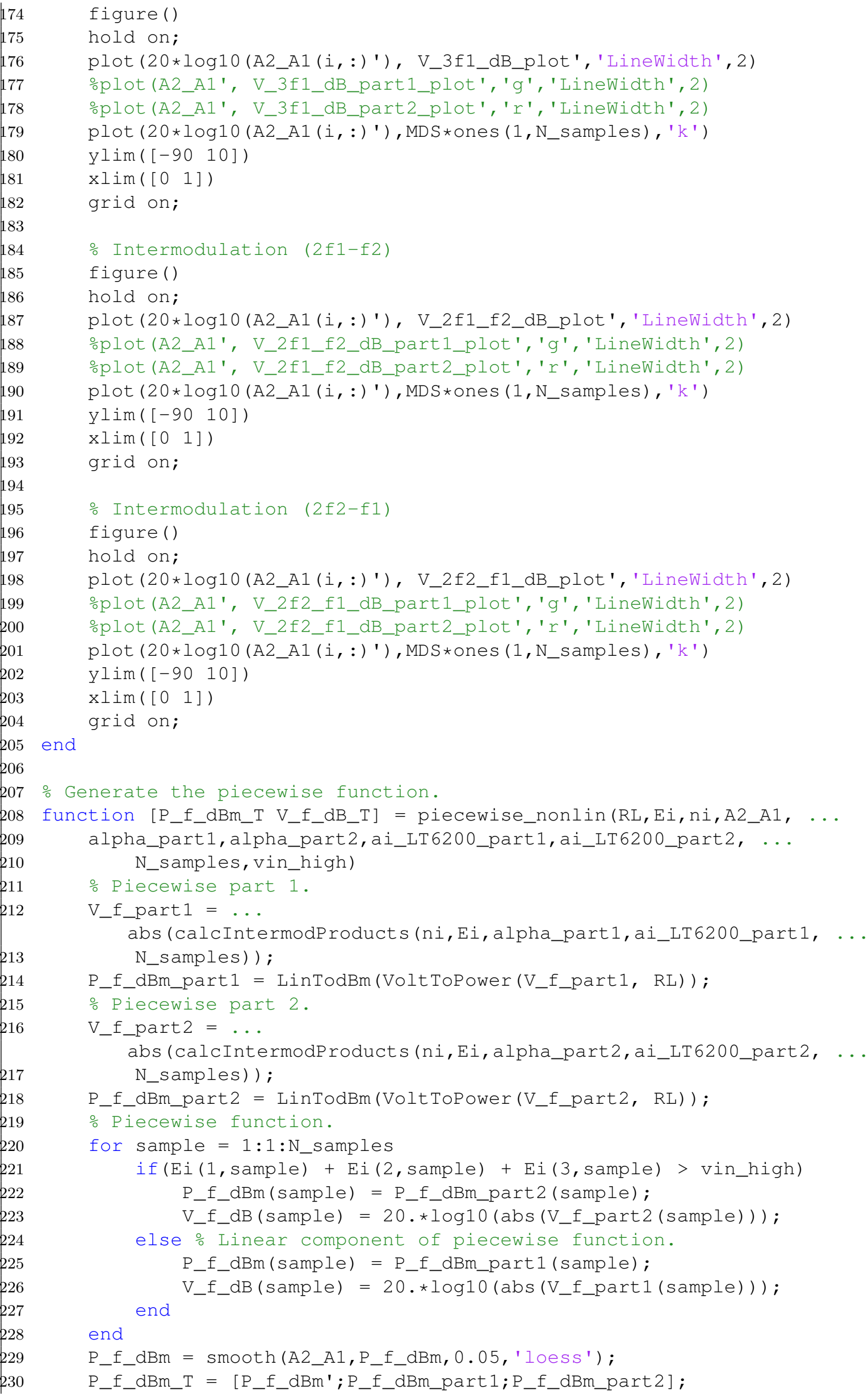


23

232

233

234 end

235

236 function [Ei] = setInputSignals (E1,N_samples, R0)

$237 \quad \operatorname{EminDB}=-80$;

$238 \quad \operatorname{EmaxDB}=20 . * \log 10(\mathrm{E} 1)$;

239 DeltaE $=(\operatorname{EmaxDB}-\operatorname{EminDB}) /($ N_samples -1$)$;

240

241

242

243

244

245

246

247

248

249

250

251

252

253

254

255

260

261

262

263

264

265

266

267

268

269

270

271

272

273

274

275

276

277

278

279

280

281

282

283

284

285

286

287

$\operatorname{Ei}(1,:)=10 . \wedge((\operatorname{EminDB}: \operatorname{DeltaE}: \operatorname{EmaxDB}) . / 20)$; Volt

$\operatorname{Ei}(1,:)=\operatorname{El}$ *ones $(1$, N_samples $) ;$ Volt

P1dBm = LinTodBm (VoltToPower $(\operatorname{Ei}(1,:)$, R0) $)$;

- Input signal 2 .

$\operatorname{Ei}(2,:)=10 \cdot \wedge((\operatorname{EminDB}: \operatorname{DeltaE}: \operatorname{EmaxDB}) \cdot / 20)$; $\frac{\circ}{\mathrm{V}} \mathrm{Volt}$

$\mathrm{E} 2$ = Ei $(2$, N_samples $)$;

$\therefore \mathrm{P} 2 \mathrm{dBm}=\operatorname{LinTodBm}(\operatorname{VoltToPower}(\mathrm{Ei}(2,:), \mathrm{RO}))$;

\% Input signal 3 .

$\mathrm{P} 3 \mathrm{dBm}=-300 ; \% \mathrm{dBm}$

$\mathrm{E} 3=$ PowerToVolt (dBmToLin $(\mathrm{P} 3 \mathrm{dBm}), \mathrm{R} 0)$;

$\operatorname{Ei}(3,:)=$ E3*ones $\left(1, N \_s a m p l e s\right)$;

end

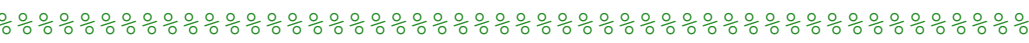

Sea, R.G.

On the computation of intermodulation products $\%$

for a power series nonlinearity.

(

o spectral component to calculate.

$\mathrm{N}=\operatorname{sum}(\operatorname{abs}(\mathrm{ni}))$;

$\mathrm{k}=\mathrm{alpha}$;

for sample = 1:1:N_samples

\% Sellect input set.

Ei $=$ Ei_set $(:$, sample);

$\div \mathrm{Eq} .8$ from proceeding letter

if $(\mathrm{N}==0)$

epsilon_N = 1 ;

else

epsilon_N $=2$;

end

o Dimension of calculation.

$\mathrm{M}=$ length (abs (ni)); amplitude

\% terms.

product_term $=1$;

for $i=1: 1: \mathrm{M}$

if $(\mathrm{ni}(\mathrm{i}) \neq 0$ ) end

end calculate the

\% Phi $(\mathrm{M}, \mathrm{L})$ component of Eq. 5.

$\div$ alpha $=\mathrm{N}+2 \mathrm{Lm}, \mathrm{Lm}=\mathrm{LO}$.

$\mathrm{LO}=\operatorname{floor}(\operatorname{abs}((\mathrm{k}-\mathrm{N}) / 2))$;

$\div \operatorname{Phi}(r, q)==\operatorname{Phi}(\mathrm{M}, \mathrm{L})$

Phi $=\operatorname{zeros}(\mathrm{M}, \mathrm{LO})$;

function V_prod_out = calcIntermodProducts (ni,Ei_set,alpha, ai, N_samples)

\% Evaluate Eq. 5 from Sea's proceeding letter.

\% Calculate first product of a sequence of input...

product_term $=$ product_term $* \operatorname{Ei}(i) \cdot \wedge a b s(n i(i)) ;$

\% Use the iterative method discribed by sea to ... 


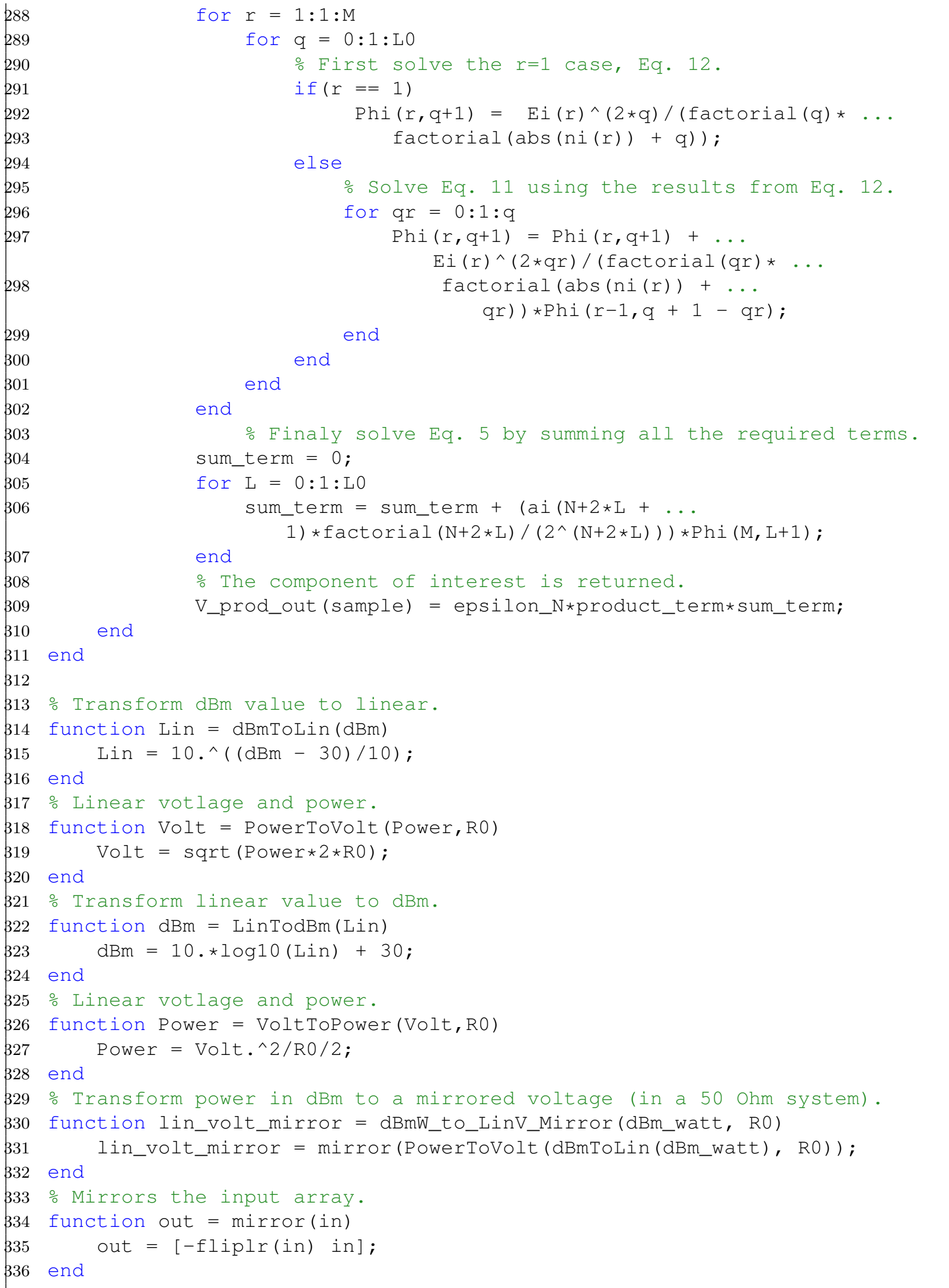




\section{B.7 Receiver calibration code}

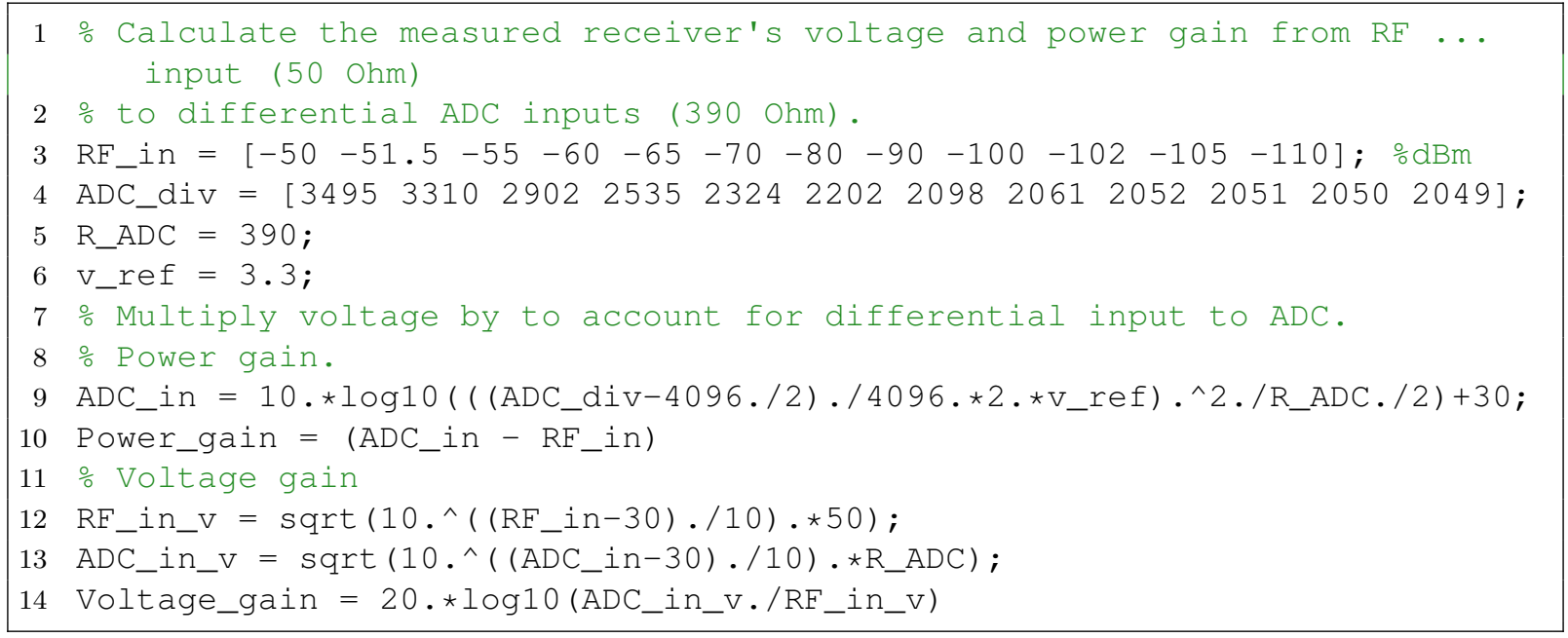




\section{Appendix C}

\section{Notation}

This appendix describes some of the notation conventions used in the thesis. The notations listed here are used as a guideline and can be found in [64] Appendix F.

\section{Voltage and current}

- Constant voltages and currents (i.e., DC voltages and currents) are designated with upper case letters and upper case indices (e.g., $V_{G S}$ or $I_{O}$ ).

- Total instantaneous voltages and currents are designated with lower case letters and upper case indices (e.g., $v_{G S}$ or $\left.i_{O}\right)$.

- Small-signal voltages and currents in the time domain are designated with lower case letters and lower case indices (e.g., $v_{g s}$ or $i_{o}$ ). These small-signal voltages and currents represent a small change in the total instantaneous value, and thus also are written as such with a $\Delta$ prefix (e.g., $\Delta v_{f s}$ or $\Delta i_{o}$ ).

- Phasers for voltages and currents (complex quantities that describe the amplitude and phase of a small-signal sinusoid) are designated with upper case letters and lower case indices (e.g., $V_{g s}$ or $I_{d}$ ).

- The peak-to-peak value (swing) of a voltage or current signal is designated with a lower case letter and $p p$ subscript (e.g., $v_{p p}$ or $i_{p p}$ ).

- The average value of a voltage or current signal is designated with a lower case letter and a horizontal bar (e.g., $\bar{v}$ or $\bar{i}$ ).

\section{Noise}

- Instantaneous noise voltages and currents in the time domain are designated with lower case letters and the index $n$ (e.g., $e_{n}, v_{n}, i_{n}$, etc.).

- The root-mean-square (rms) noise voltages and currents that have the units of volt per square hertz $(\mathrm{V} / \sqrt{\mathrm{Hz}})$ are represented by a lower case letter and upper case subscript (e.g., $v_{N}, i_{N}$, etc.).

- Mean-square noise voltages and currents, measured in a given bandwidth, are designated with $\overline{v_{n}^{2}}$ and $\overline{i_{n}^{2}}$, respectively. These quantities also are referred to as noise powers for short. The mean indicates either the time average (for stationary noise) or the ensemble average (for non-stationary noise), as appropriate. These noise 
quantities can be calculated as the integral of a spectral density over the given bandwidth.

- Root-mean-square noise voltages and currents, measured in a given bandwidth, are designated with $\overline{v_{n}}$ and $\overline{i_{n}}$, respectively. These quantities are the square roots of the mean-square noise quantities.

- The power spectral densities for voltages and currents are designated with $V_{n}^{2}$ and $I_{n}^{2}$, respectively. These quantities also are referred to as power spectra for short. Usually the power spectral densities are one sided, unless specified otherwise, and thus directly represent the noise power in a $1 \mathrm{~Hz}$ bandwidth. Power spectral densities can be calculated as the Fourier transform of the autocorrelation function of the time domain signal.

- The root spectral densities for voltages and currents are designated with $V_{n}$ and $I_{n}$, respectively. These quantities are the square roots of the power spectral densities and represent the rms noise voltage or current in a $1 \mathrm{~Hz}$ bandwidth. 


\section{Appendix D}

\section{Miscellaneous measurements}

\section{Coaxial cable insertion loss}

The insertion loss of a one meter long coaxial cable was measured using a Rohde \& Schwartz : $Z V B-8$ vector network analyser.

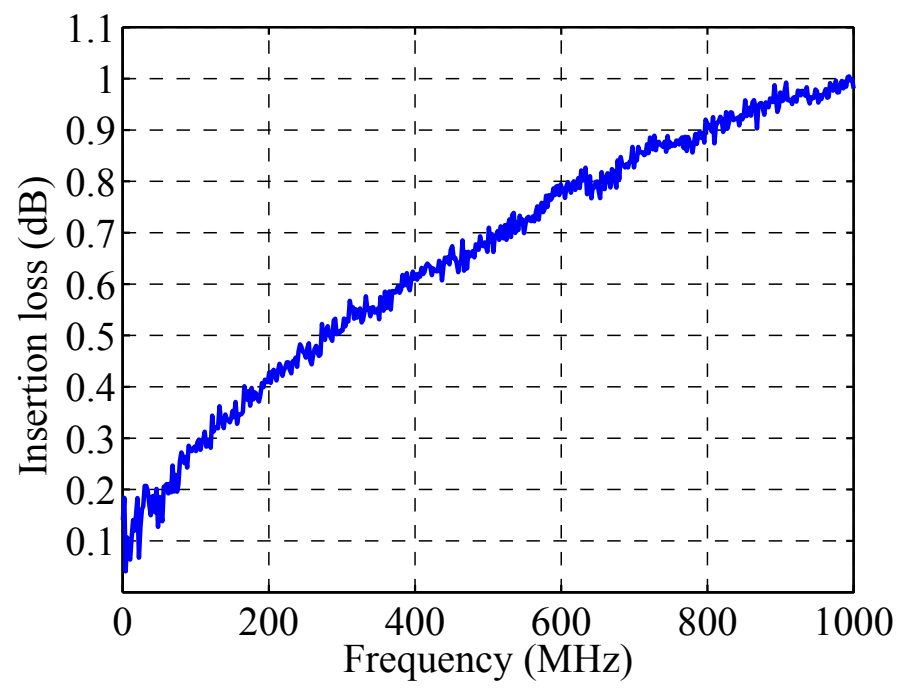

Figure D.1: One meter coaxial cable insertion loss vs frequency. 


\section{Appendix E}

\section{Datasheet information}

\section{Balun}

Tables, E.1, E.2 and E.3, contain relevant datasheet information on the Mini-Circuits RF transformer's, TCM1-63AX+, TC1-1-13M+, and TCM9-1+.

Table E.1: TCM1-63AX+, Mini-circuits RF 1:1 Impedance transformer - 10 to $6000 \mathrm{MHz}$, insertion loss data [65]

\begin{tabular}{c|c}
\hline \hline Insertion loss $[\mathrm{dB}]$ & frequency $[\mathrm{MHz}]$ \\
\hline 1.5944 & 300 \\
1.5667 & 400 \\
1.5389 & 500 \\
1.5111 & 600 \\
1.4833 & 700 \\
1.4556 & 800 \\
1.4278 & 900 \\
1.4 & 1000 \\
\hline
\end{tabular}

Table E.2: TC1-1-13M+, Mini-circuits RF 1:1 Impedance transformer - 4.5 to $3000 \mathrm{MHz}$, insertion loss data [51]

\begin{tabular}{c|c}
\hline \hline Insertion loss $[\mathrm{dB}]$ & frequency $[\mathrm{MHz}]$ \\
\hline 0.372 & 300 \\
0.416 & 400 \\
0.46 & 500 \\
0.504 & 600 \\
0.548 & 700 \\
0.592 & 800 \\
0.636 & 900 \\
0.68 & 1000 \\
\hline
\end{tabular}


Table E.3: TCM9-1+, Mini-circuits RF 9:1 Impedance transformer - 2 to $280 \mathrm{MHz}$, insertion loss data [66]

\begin{tabular}{c|c}
\hline \hline Insertion loss $[\mathrm{dB}]$ & frequency $[\mathrm{MHz}]$ \\
\hline 1.09 & 2 \\
0.87 & 5 \\
0.82 & 10 \\
0.87 & 30 \\
0.90 & 50 \\
\hline
\end{tabular}




\section{Appendix F}

\section{Schematics and PCB layouts}

The schematics and PCB layouts are done using Altium's PCB design tools.

\section{F.1 Demodulator baseband circuit}

\section{F.1.1 Schematics}

Figure F.1 shows the circuitry connected to the demodulator's baseband ports. This circuit forms part of the receiver after down conversion to the IF. The sub-circuit schematics, represented by dark grey blocks in Fig. F.1 are provided in Figs. F.2 to F.6.

\section{F.1.2 PCB}

The demodulator's baseband circuit PCB layout is illustrated in Figs. F.7 and F.8.

\section{F.2 Intermediate frequency offset local oscillator circuit}

\section{F.2.1 Schematics}

The circuitry connected between the modulator's baseband ports and the $125 \mathrm{MHz}$ oscillator are shown in Fig. F.9. This circuit introduces the IF offset to the LO signal. The sub-circuit schematics are illustrated in Figs. F.10 to F.12.

\section{F.2.2 PCB}

The top and bottom views to the IF offset LO PCB layout is presented in Fig. F.13. 


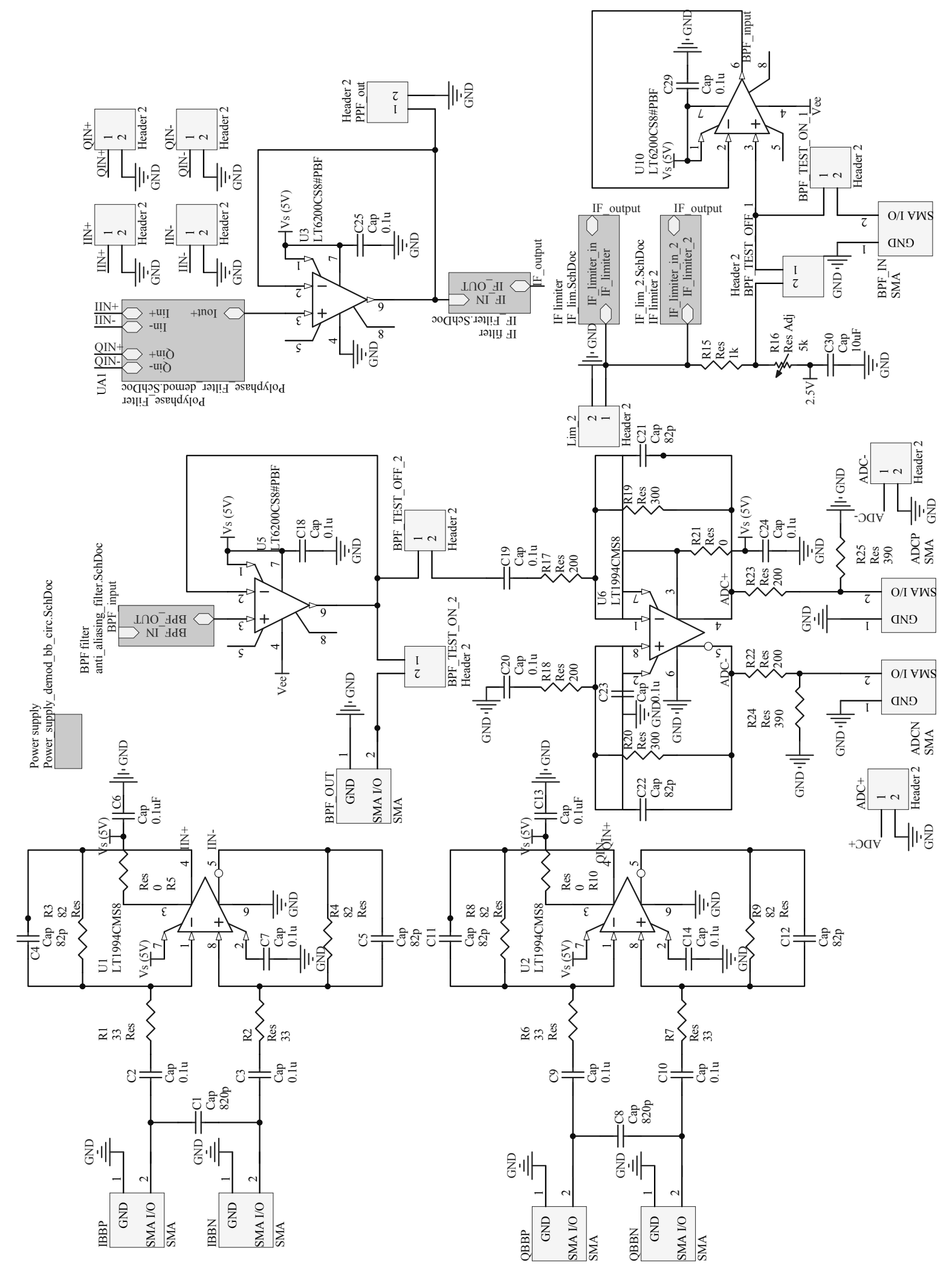

Figure F.1: The demodulator's baseband output circuitry with sub-circuit blocks. 


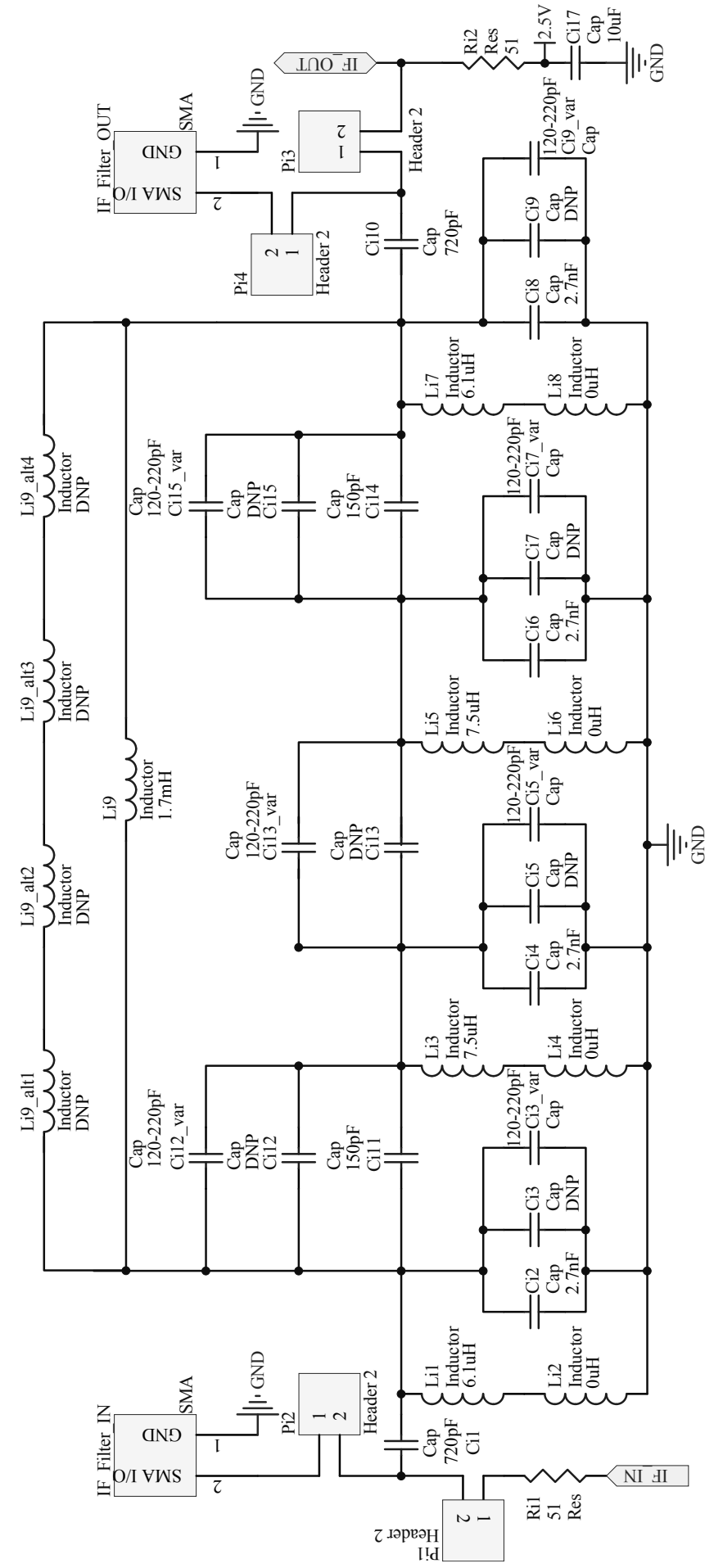

Figure F.2: IF-filter schematic. 


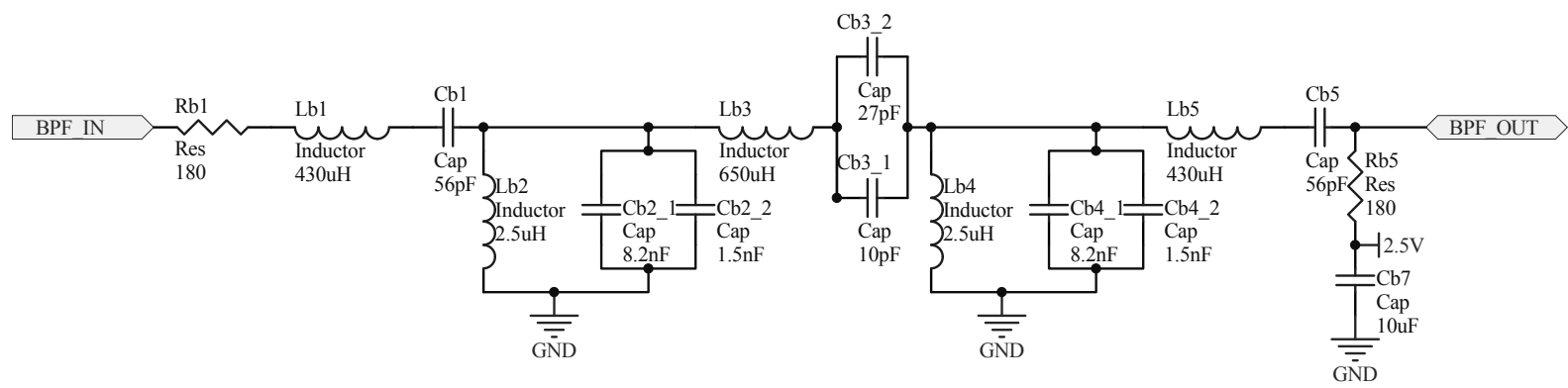

Figure F.3: Anti-aliasing filter schematic.

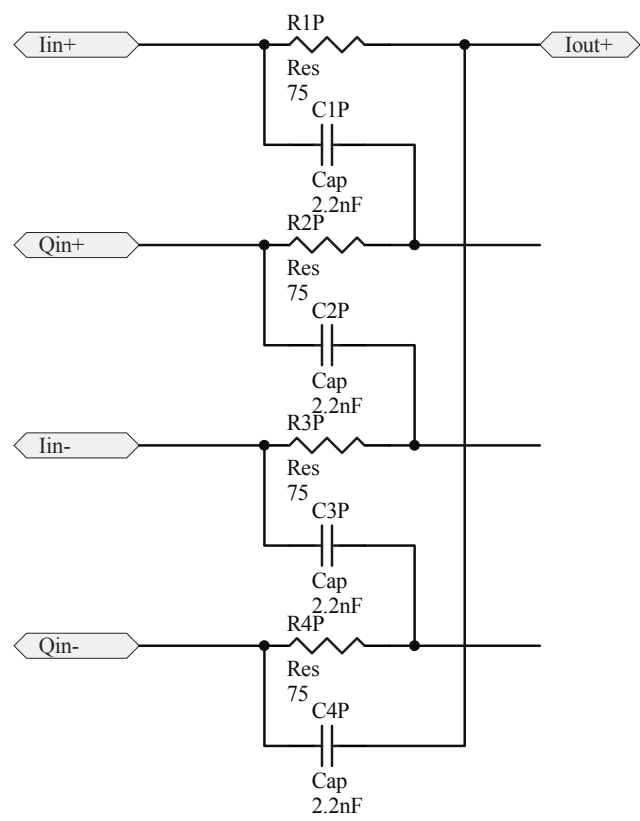

Figure F.4: Passive polyphase filter schematic. 


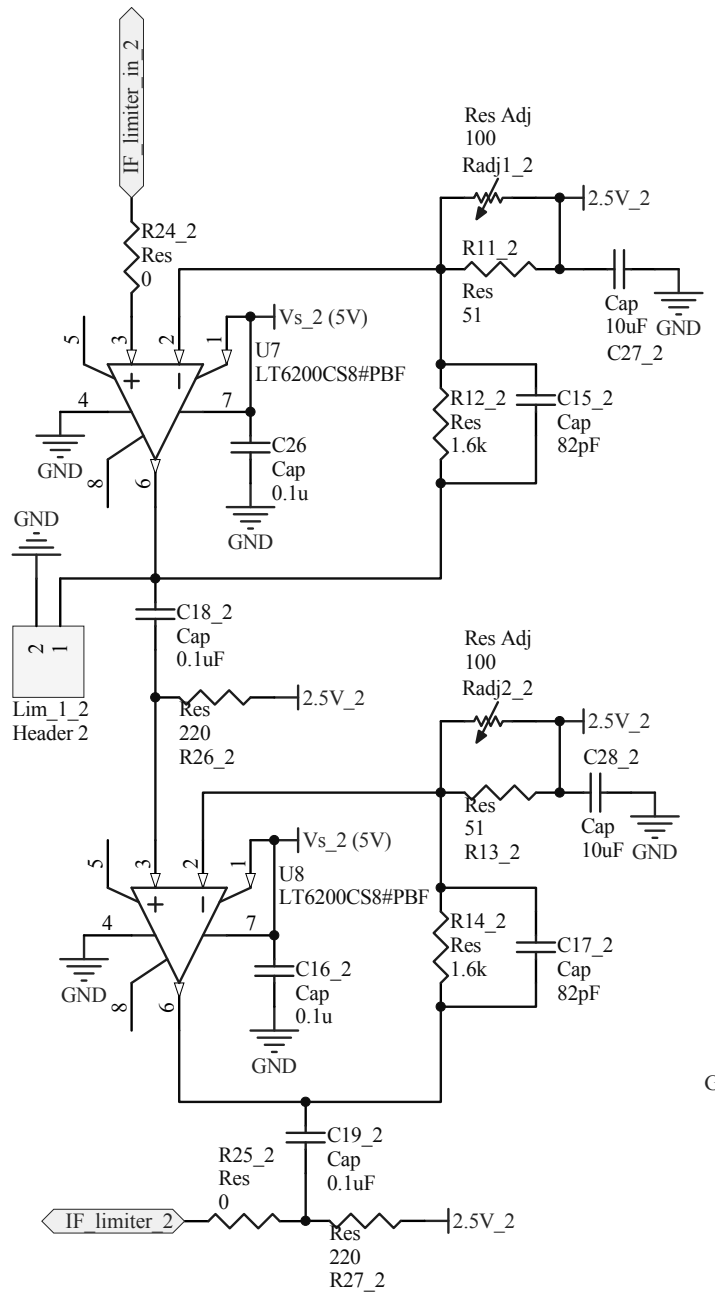

(a)

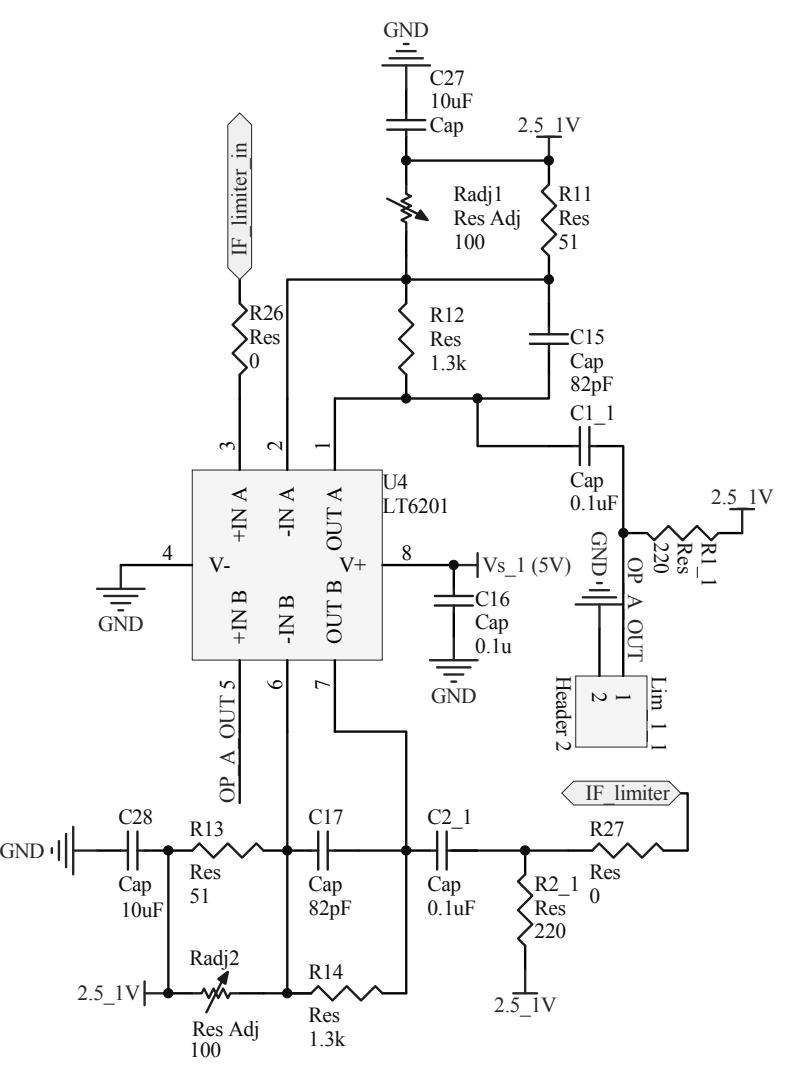

(b)

Figure F.5: Two high voltage gain operational amplifier schematics (a) and (b) are presented, but only (b) is populated on the PCB. 


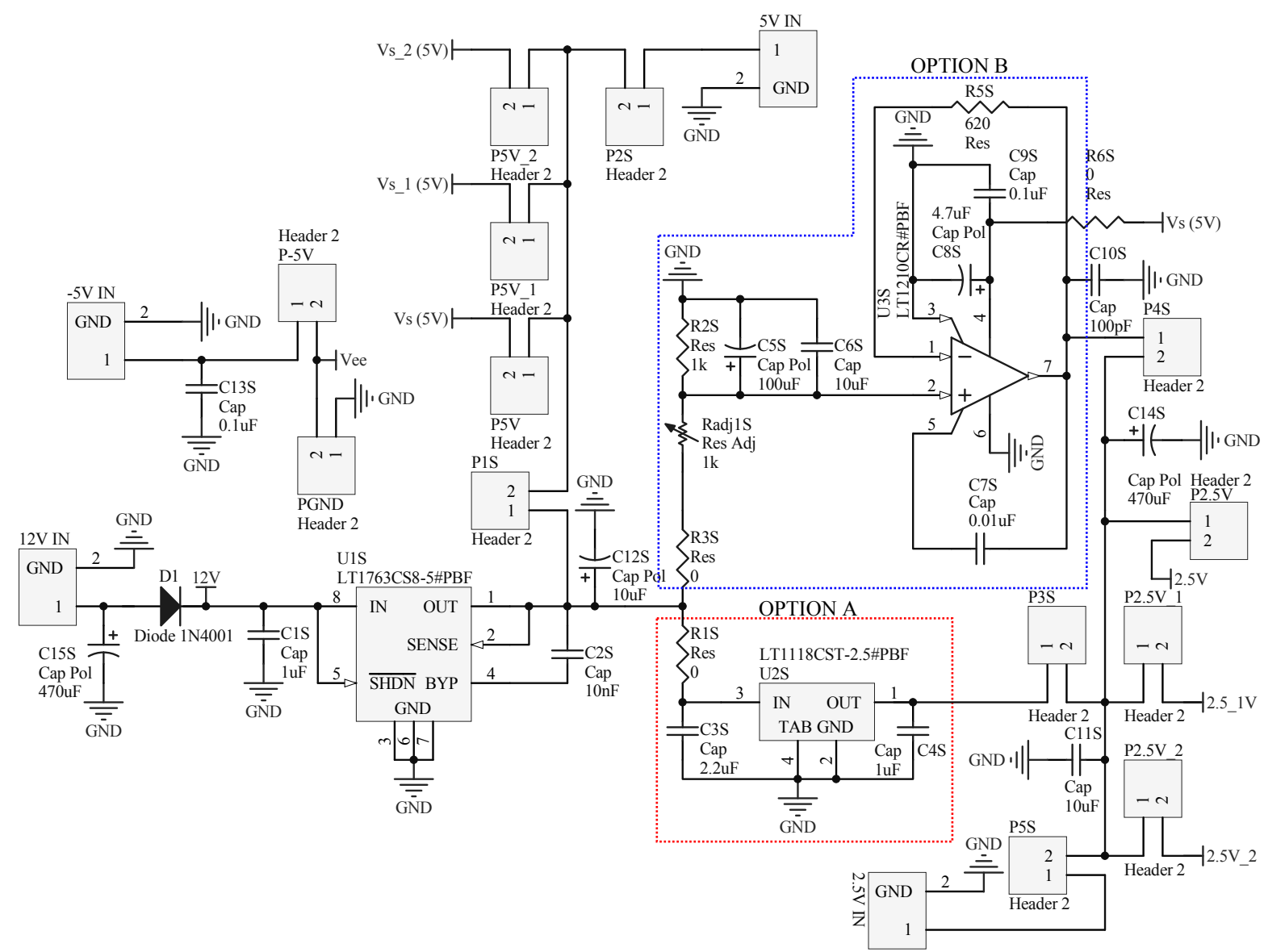

Figure F.6: Two regulator options are illustrated on the schematic, but option A is populated on the PCB. 


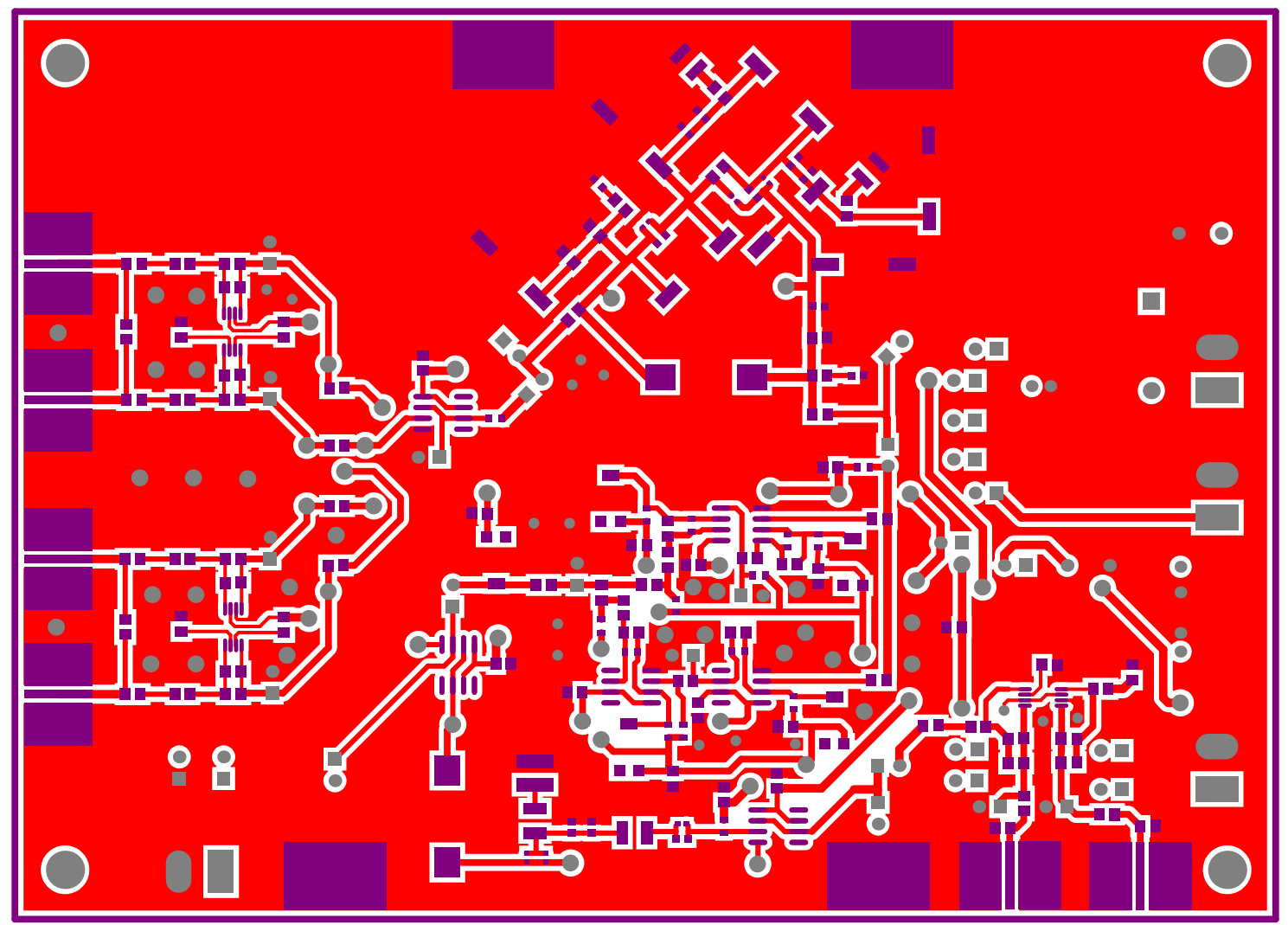

Figure F.7: Demodulator baseband circuit PCB top layer.

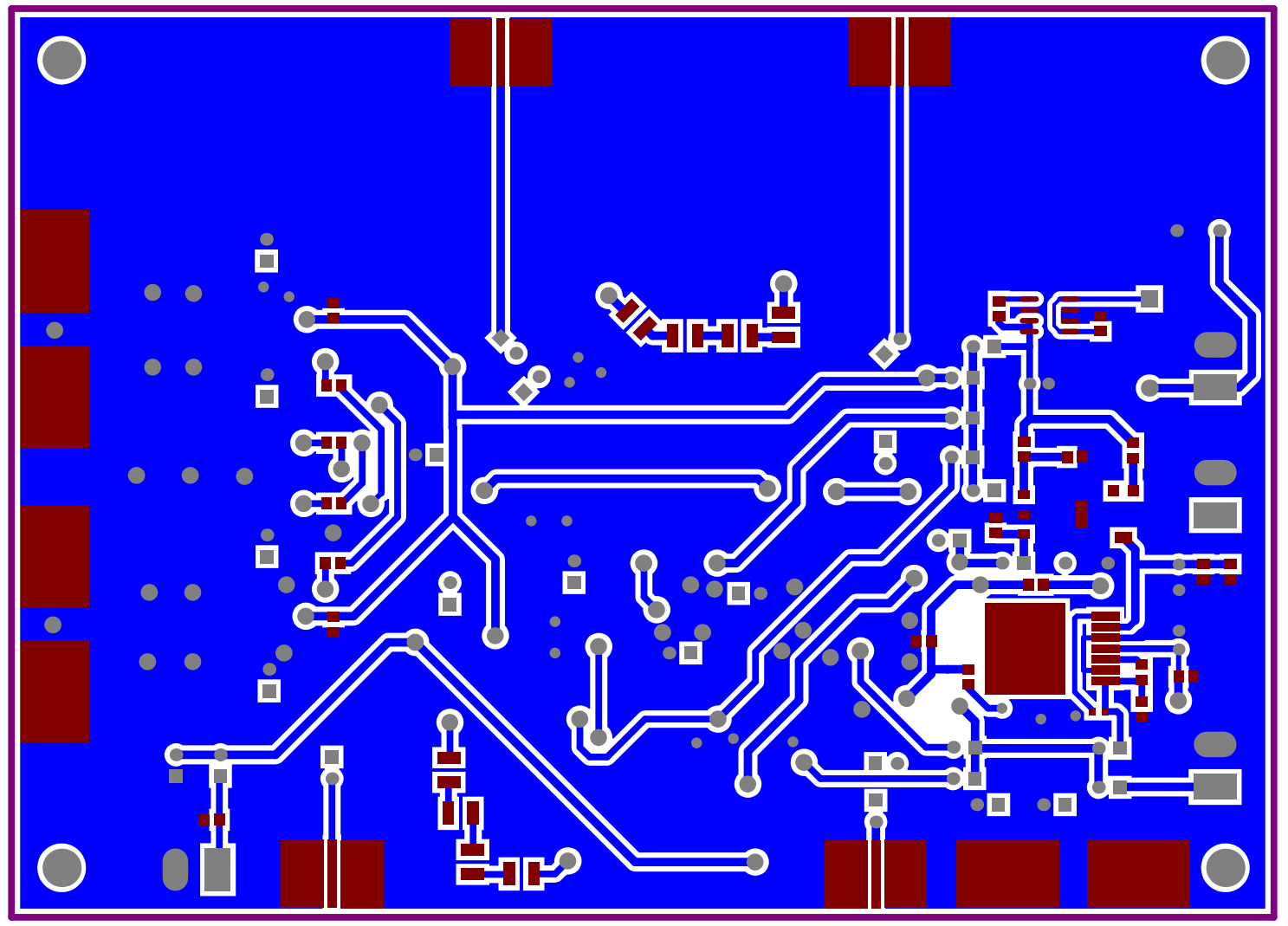

Figure F.8: Demodulator baseband circuit PCB bottom layer 


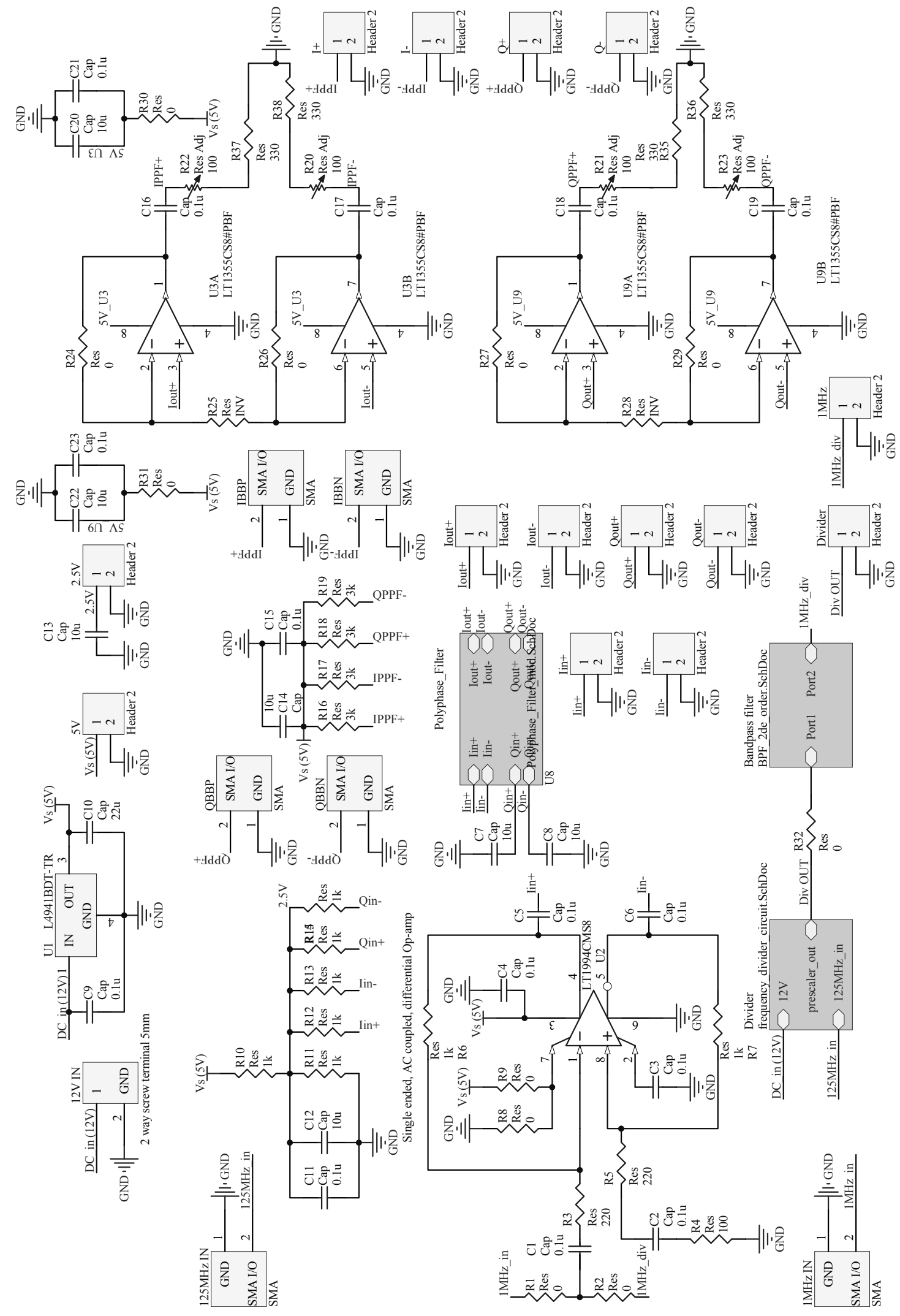

Figure F.9: IF offset local oscillator circuit schematic. 


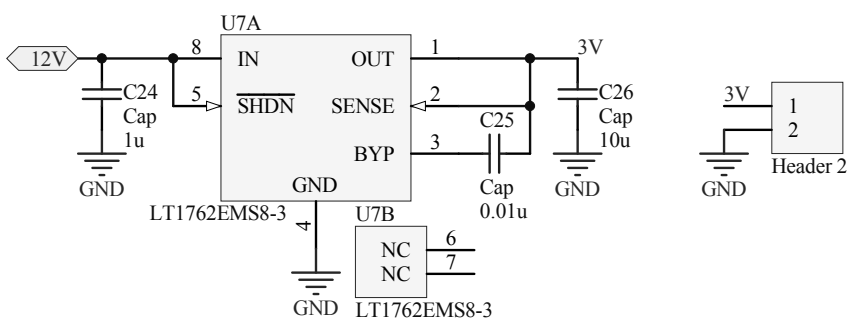

(a)

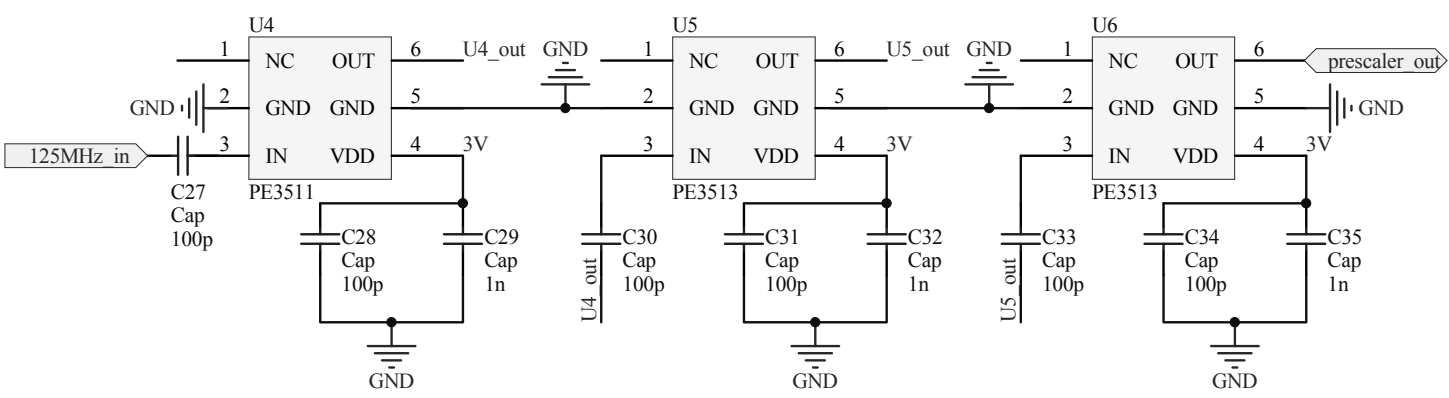

(b)

Figure F.10: Voltage regulator (a) and 128 prescaler schematic (b).

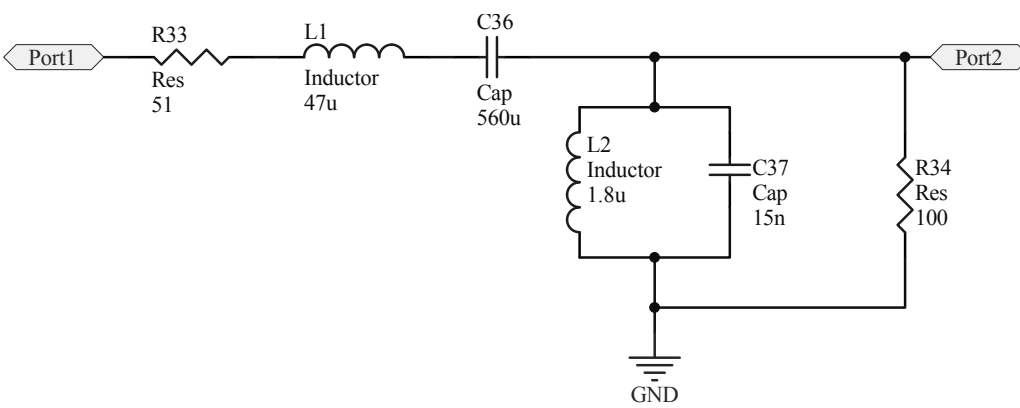

Figure F.11: Prescaler bandpass filter schematic.

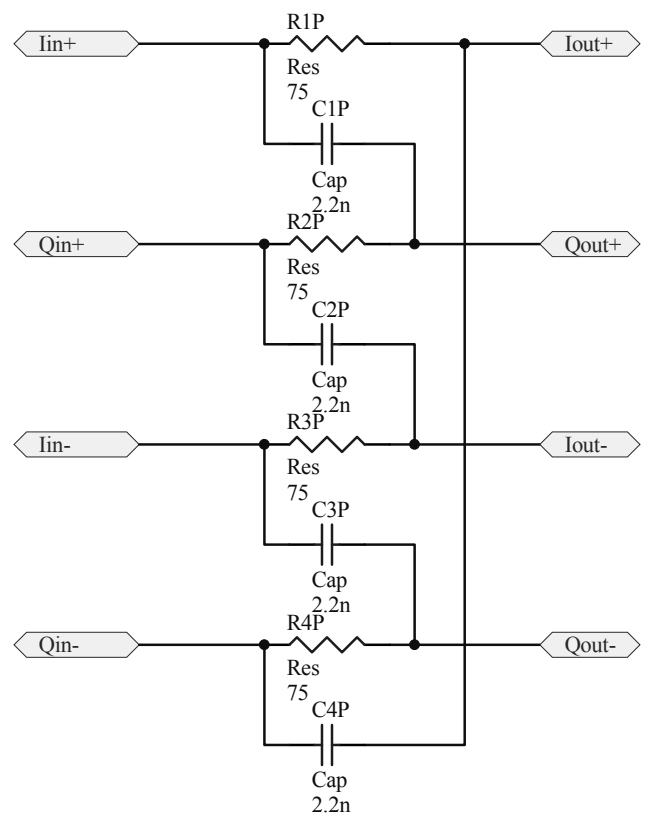

Figure F.12: Schematic for the passive polyphase filter with differential inputs and outputs. 


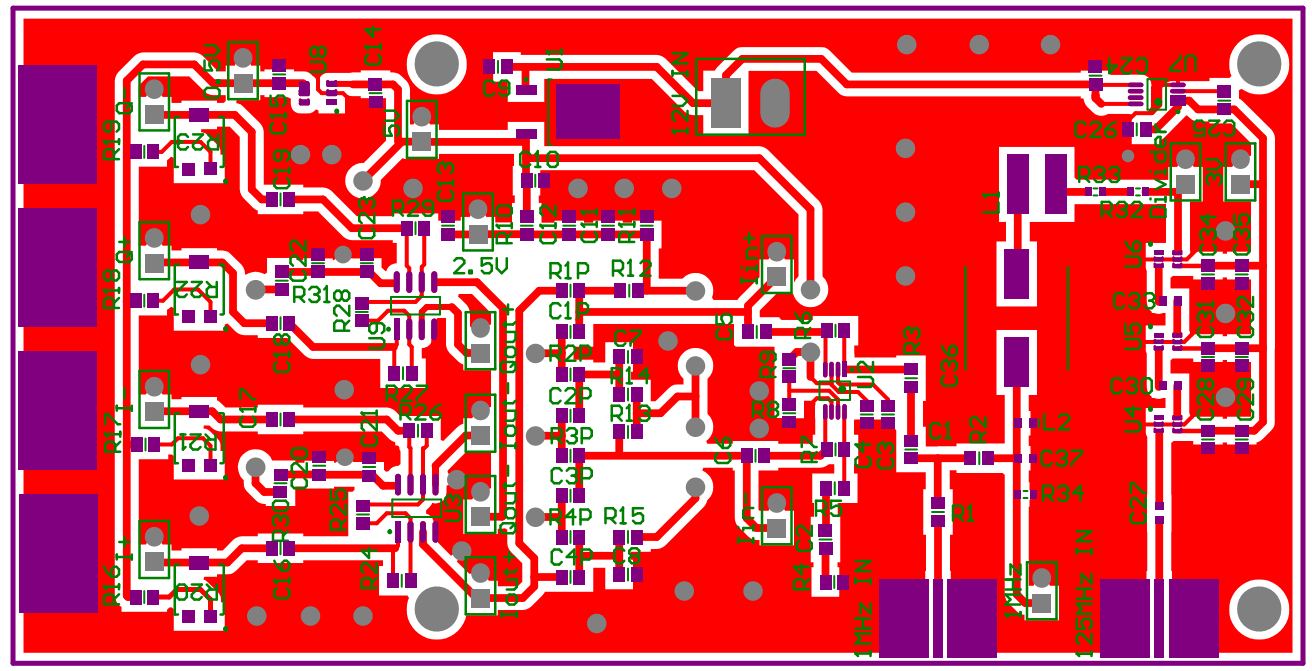

(a)

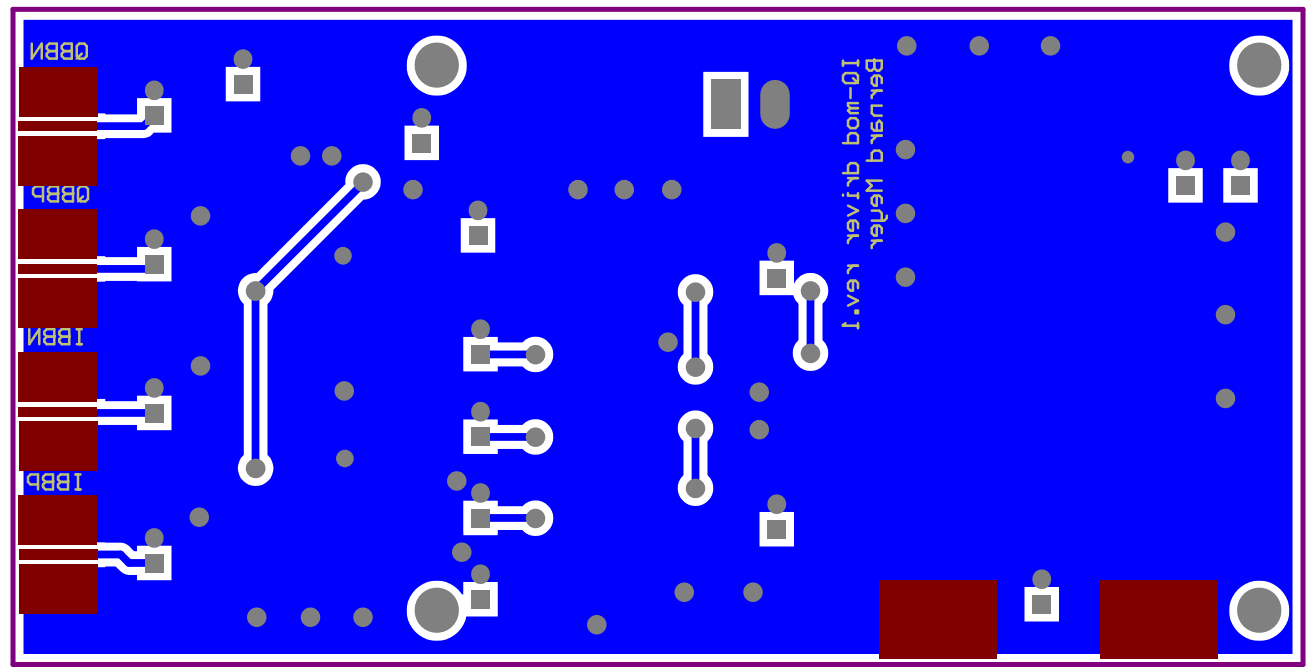

(b)

Figure F.13: The IF offset local oscillator circuit's PCB layout, where (a) and (b) are the top and bottom views respectively. 


\section{Appendix G}

\section{Photos}

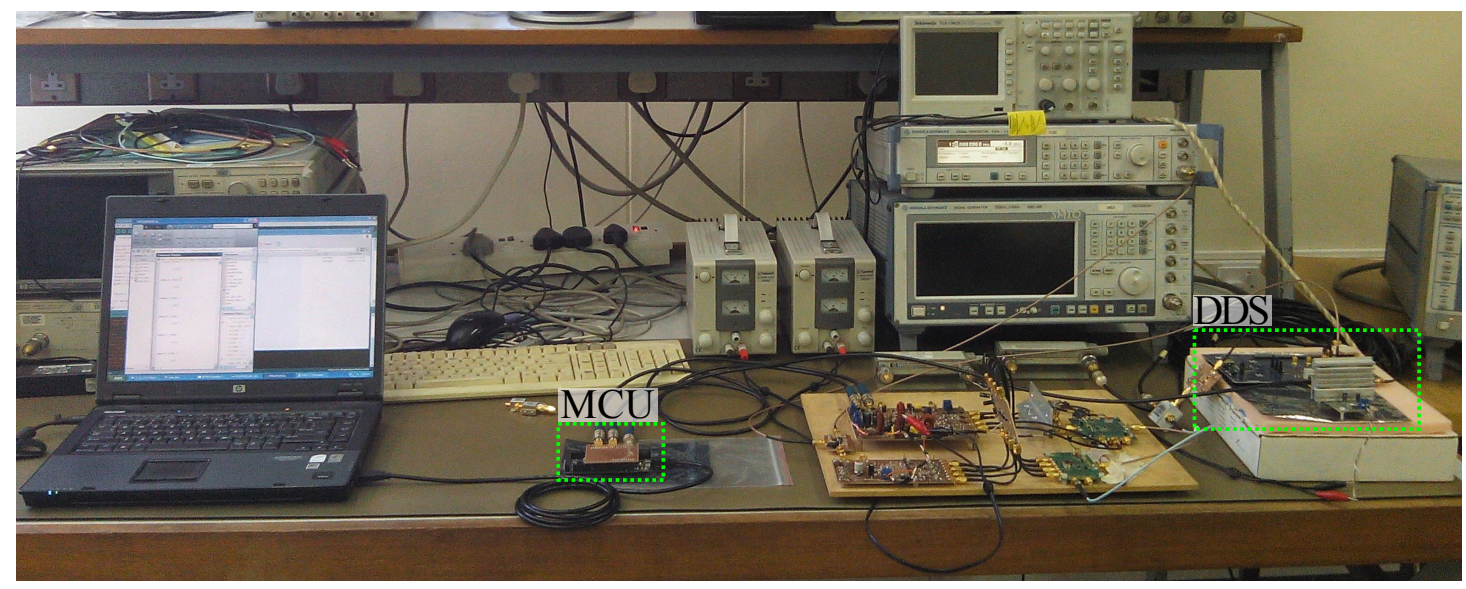

Figure G.1: Cable measurement set-up.

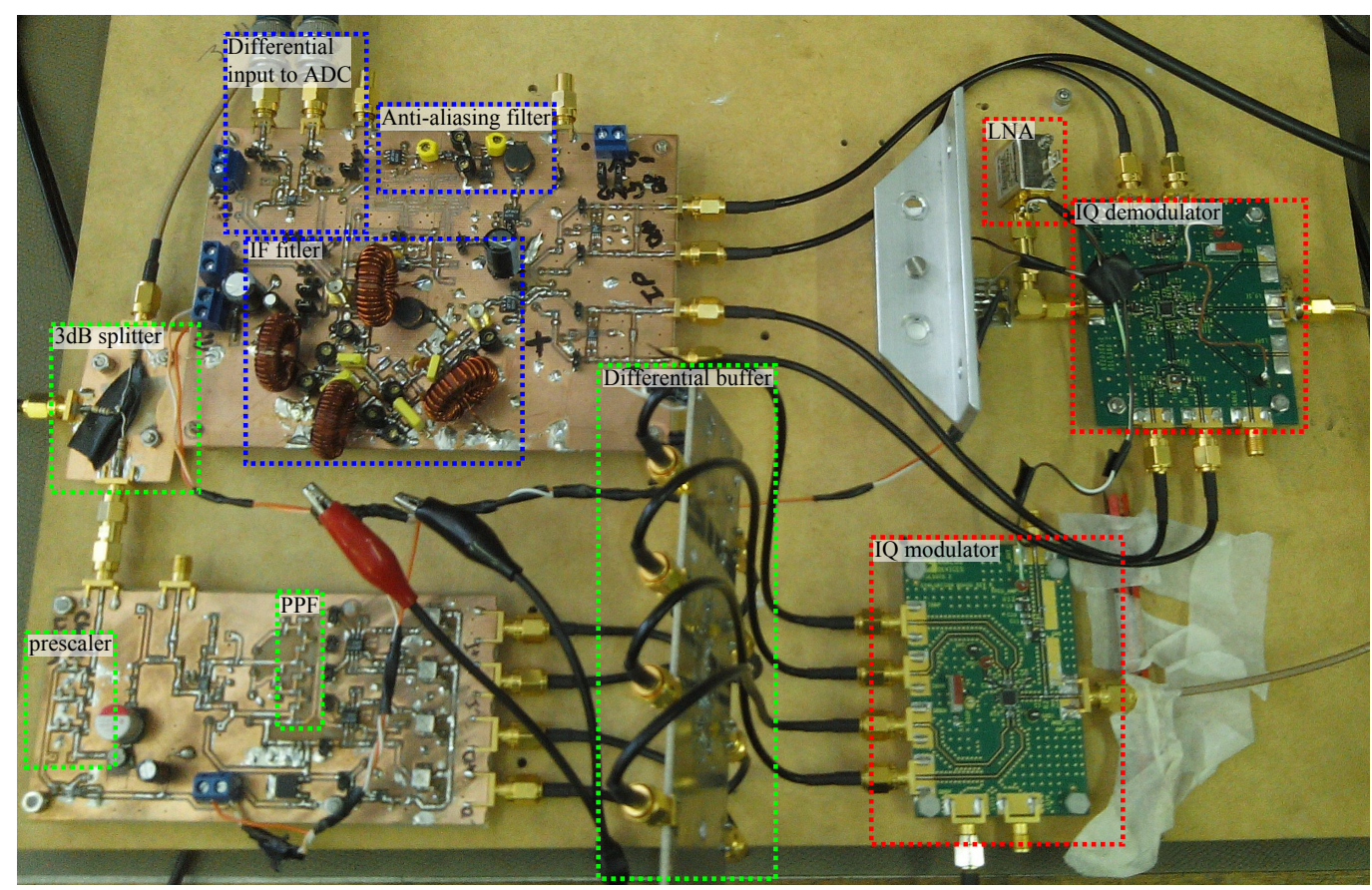

Figure G.2: Constructed modular IF FMCW radar. 


\section{Bibliography}

[1] M. I. Skolnik, Introduction to Radar Systems. McGraw-Hill Book Company, Inc, 1962.

[2] D. J. Daniels, Ground Penetrating Radar, 2nd ed. The Institution of Engineering and Technology, 2007.

[3] A. Howe, Ground Penetrating Radar for the parameterisation of subsurface hydrological properties. Department of Geography King's College London, Sept. 2000.

[4] T. S. Rappaport, Wireless Communications: Principles and Practice, 2nd ed. Prentice Hall, Jan. 2002.

[5] G. M. Brooker, "Understanding millimetre wave fmcw radars," 1st International Conference on Sensing Technology, pp. 152-157, Nov. 2005.

[6] W. F. Egan, Practical RF System Design, 1st ed. John Wiley and Sons,Inc, 2003.

[7] 3.5 GSPS Direct Digital Synthesizer with 12-Bit DAC, Analog Devices, 2012, Rev. C. [Online]. Available: www.analog.com

[8] D. M. Pozar, Microwave Endineering, 4th ed. John Wiley and Sons,Inc, 2011.

[9] J. Kaukovouri, K. Stadius, J. Ryynänen, and K. A. I. Halonen, "Analysis and design of passive polyphase filters," Circuits and Systems I: Regular Papers, IEEE Transactions, vol. 55, no. 10, Nov. 2008.

[10] A. Mashhour, W. Domino, and N. Beamish, "On the Direct Conversion Receiver," Microwave Journal, June 2001, Tutotial - A detailed look at the characteristics of the direct conversion receiver.

[11] W. R. Fayrouz Haddad, Lakhdar Zaïd and O. Frioui, Polyphase Filter Design Methodology for Wireless Communication Applications, Mobile and Wireless Communications Network Layer and Circuit Level Design. University of Provence Marseille, France. [Online]. Available: http://www.intechopen.com/ books/mobile-and-wireless-communications-network-layerand-circuit-level-design/ polyphase-filter-design-methodology-for-wireless-communication-applications

[12] J. Crols and M. S. J. Steyaert, "Low-IF Topologies for High-Performance Analog Front Ends of Fully Integrated Receivers," IEEE Transcations on circuits and systems II: Analog and digital signal processing, vol. 45, no. 3, 1998.

[13] B. Razavi, "Architectures and circuits for rf cmos receivers," IEEE Custom Integrated Circuits Conference, pp. 393-400, 1998. 
[14] J. C. Rudell, J. J. Cho, G. Chien, F. Brianti, J. A. Weldon, and P. R. Gray, "A $1.9 \mathrm{GHz}$ wideband IF double conversion CMOS receiver for cordless telephonse applications," IEEE journal of Solid State Circuits, vol. 32, no. 12, pp. 2071-2088, 1997.

[15] $400 \mathrm{MHz}$ to $6 \mathrm{GHz}$ Quadrature Demodulator: ADL5380, Analog Devices, 2009. [Online]. Available: www.analog.com

[16] J. Chow, "Rf image-reject receivers," Electrical and Computer Engineering Department, University of Toronto, Toronto, Ontario, Canada, 2002.

[17] Fundamentals of Sampled Data Systems, Analog Devices, Application note, AN-282.

[18] B. P. Lathi and Z. Ding, Modern Digital and Analog Communication Systems, 4th ed., A. S. Sedra, Ed. Oxford University Press, 2010.

[19] Understanding Single-Ended, Pseudo-Differential and Fully-Differential ADC Inputs, Maxim Integrated Products Inc, 2002, application note 1108.

[20] High-Speed, Analog-to-Digital Converter Basics, Texas instruments, Jan. 2011, Application Report, SLAA510.

[21] What the Nyquist Criterion Means to Your Sampled Data System Design, Analog Devices, 2009, Tutorial, MT-002.

[22] P. Poshala, Why Oversample when Undersampling can do the Job?, Texas Instruments, July 2013, Application Report: SLAA594A.

[23] W. Kester, Data Conversion Handbook. Analog Devices Inc, Newnes, 2004.

[24] L. Green, "RF-inductor modeling for the 21st century," EDN Network, Sept. 2001, Accessed: 15 Dec., 2014. [Online]. Available: http://www.edn.com/design/ components-and-packaging/4344111/RF-inductor-modeling-for-the-21st-century

[25] AVX Surface Mount Ceramic Capacitor Products, AVX A Kyocera Group Company, Accessed: 15 Aug., 2015. [Online]. Available: http://www.avx.com/docs/ masterpubs/smccp.pdf

[26] J. S. Lee, Microwave Resonator Filters for Advanced Wireless Systems. The University of Michigan, 2009.

[27] R. J. Cameron, "Advanced Filter Synthesis," IEEE microwave magazine, pp. 42-61, Oct. 2011.

[28] R. J. Cameron, R. Mansour, and C. M. Kudsia, Microwave Filters for Communication Systems : Fundamentals, Design and Applications. Wiley, Aug. 2007.

[29] R. J. Cameron, "General coupling matrix synthesis methods for chebyshev filtering functions," IEEE transaction on microwave theory and techniques, vol. 47, no. 4, pp. 433-442, Apr. 1999.

[30] R. J. Cameron, "Advanced coupling matrix synthesis techniques for microwave filters," Microwave Theory and Techniques, IEEE Transactions, vol. 51, pp. 1-10, Jan. 2003. 
[31] The IEEE Standard Dictionary of Electrical and Electronics Terms, 4th ed. Institute of Electrical and Electronics Engineers, Jan. 1988.

[32] C. A. Motchenbacher and J. A. Connelly, Low-Noise Electronics System Design, 1st ed. John Wiley and Sons, Inc, 1993.

[33] G. Vasilescu, Electronic Noise and Interfering Signals: Principles and Applications. Springer, 1999.

[34] A. Oppenheim and G. Verghese. MIT (Massachusetts Institute of Technology) OpenCourseWare: 6.011 Introduction to Communication, Control. Spring 2010. Accessed: 3 Nov., 2014. License: Creative Commons BY-NC-SA. [Online]. Available: http://ocw.mit.edu/courses/electrical-engineering-and-computer-science/ 6-011-introduction-to-communication-control-and-signal-processing-spring-2010

[35] Noise Analysis in Operational Amplifier Circuits, Texas instruments, 2007, Application Report, SLVA043B. [Online]. Available: http://www.ti.com/lit/an/slva043b/ slva043b.pdf

[36] J. R. Ragazzini and S. S. L. Chang, "Noise and random processes," Proceedings of the IRE, vol. 50, no. 5, pp. 1146-1151, May 1962.

[37] B. M. Oliver, "Thermal and quantum noise," Proceedings of the IEEE, vol. 53, no. 5, pp. 436 - 454, June 2005.

[38] J. B. Johnson, "Thermal agitation of electricity in conductors," The American Physical Society, 1928.

[39] H. Nyquist, "Thermal agitation of electric charge in conductors," Physical Review, vol. 32, pp. 110 - 113, July 1928.

[40] M. T. Lebenbaum, "Some Perspectives on Receiver Noise Performance and Its Measurement," Microwave J., vol. 16, Jan 1973.

[41] P. Lee, Low noise amplifier selection guide for optimal noise performance, Analog Devices, P.O. Box 9106, Norwood, M.A., Application note, AN-940.

[42] T. J. Karras, "Equivalent noise bandwidth analysis from transfer functions," Nasa technical note: NASA TN D-2842, Nov. 1965, Goddard space flight center, Greenbelt, $\mathrm{Md}$.

[43] G. D. Vendelin, A. M. Pavio, and U. L. Rohde, Microwave Circuit Design using Linear and Nonlinear Techniques. John Wiley and Sons,Inc, Apr. 2005.

[44] W. M. Leach. Georgia Institute of Technology: ECE 4391 Electromagnetic Compatability. Summer 2004. Accessed: 6 Nov., 2014. [Online]. Available: http://users.ece.gatech.edu/mleach/ece4391/noise_a.pdf

[45] Op amp noise figure: Don't be mislead, Analog Devices, Oct. 2008, Tutorial, MT-052.

[46] 165MHz, Rail-to-Rail Input and Output, 0.95nV/ $\sqrt{\mathrm{Hz}}$ Low Noise, Op Amp Family, LT6200/LT6200-5 LT6200-10/LT6201, Linear Technology, Dec. 2011, rev. F.

[47] D. A. Neamen, Microelectronincs, Circuit Analysis and Design, 3rd ed. McGrawHill, 2007. 
[48] Op Amp Total Output Noise Calculations for Single-Pole System, Analog Devices, Tutorial, MT-049.

[49] AN-1719 Noise figure analysis fully differential amplfier, Texas instruments, May 2013, Application Report, AN-1719.

[50] Low Noise Amplifier, $50 \Omega 40$ to $2600 \mathrm{MHz}$, Mini-Circuits, P.O. Box 35166, Brooklyn, NY, Datasheet ZX60-P105LN+.

[51] Surface Mount 50 Ohm RF Transformer 4.5 to 3000 MHz - TC1-1-13M+, MiniCircuits, P.O. Box 35166, Brooklyn, NY, Datasheet TC1-1-13M+.

[52] J. Ladvánszky and G. Schultes, "Noise Figure Minimization of RC Polyphase Filters," Proceedings European Conference on Circuit Theory and Design, Sept. 2003.

[53] H. A. Haus and R. B. Adler, "Optimum noise performance of linear amplifiers," Proceedings of the IRE, pp. 1517-1533, 1958.

[54] Understand SINAD, ENOB, SNR, THD, THD + N, and SFDR so You Don't Get Lost in the Noise Floor, Analog Devices, 2009, Tutorial, MT-003.

[55] S. Voinigescu, High-Frequency integrated circuits. Cambridge University Press, 2013.

[56] B. A. Fette, R. Aiello, P. Chandra, D. M. Dobkin, A. Bensky, D. B. Miron, D. Lide, F. Dowla, and R. Olexa, RF and Wireless Technologies: Newnes Know It All. Newnes Newton, 2007.

[57] Specifications for Vector Signal Generator R\&S SMIQ, Rohde \& Schwarz, München Germany, July 2002, Accessed: 24 Nov., 2014. [Online]. Available: https: //www.fer.unizg.hr/_download/repository/R_S_SMIQ03B_specs.pdf

[58] T. Dekker. MathWorks - fzero function. Accessed: 1 Dec., 2015. [Online]. Available: http://www.mathworks.com/help/matlab/ref/fzero.html

[59] R. G. Sea and A. G. Vacroux, "An algebraic formula for amplitudes of intermodulation products involving an arbitrary number of frequencies." Proc. IEEE (Letters), vol. 56, pp. 1388-1389, Aug. 1968.

[60] R. G. Sea and A. G. Vacroux, "On the computation of intermodulation products for a power series nonlinearity." Proc. IEEE (Letters), vol. 57, pp. 337-338, Mar. 1969.

[61] (2009, June) The Fundamentals of FFT-Based Signal Analysis and Measurement in LabVIEW and LabWindows/CVI. National Instruments. Accessed: 19 Sept., 2015. [Online]. Available: http://www.ni.com/white-paper/4278/en/

[62] J. J. Grobbelaar, Phase Noise Measurement. Stellebosch University, 2011.

[63] Fundamentals of RF and Microwave Noise Figure Measurements, Agilent Technologies, Application note, 57-1.

[64] E. Säckinger, Broadband Circuits for Optical Fiber Communication. John Wiley and Sons,Inc, Apr. 2005.

[65] Surface Mount 50 Ohm RF Transformer 10 to $6000 \mathrm{MHz}$ - TCM1-63AX+, MiniCircuits, P.O. Box 35166, Brooklyn, NY, Datasheet TCM1-63AX+. 
[66] Surface Mount 50 Ohm RF Transformer 2 to $280 \mathrm{MHz}$ - TCM1-63AX+, MiniCircuits, P.O. Box 35166, Brooklyn, NY, Datasheet TCM9-1+. 